\title{
Small fiber neuropathy and sodium channels : a paradigm shift
}

Citation for published version (APA):

Hoeijmakers, J. G. J. (2014). Small fiber neuropathy and sodium channels : a paradigm shift. [Doctoral Thesis, Maastricht University]. Datawyse / Universitaire Pers Maastricht. https://doi.org/10.26481/dis.20140214jh

Document status and date:

Published: 01/01/2014

DOI:

10.26481/dis.20140214jh

Document Version:

Publisher's PDF, also known as Version of record

\section{Please check the document version of this publication:}

- A submitted manuscript is the version of the article upon submission and before peer-review. There can be important differences between the submitted version and the official published version of record.

People interested in the research are advised to contact the author for the final version of the publication, or visit the DOI to the publisher's website.

- The final author version and the galley proof are versions of the publication after peer review.

- The final published version features the final layout of the paper including the volume, issue and page numbers.

Link to publication

\footnotetext{
General rights rights.

- You may freely distribute the URL identifying the publication in the public portal. please follow below link for the End User Agreement:

www.umlib.nl/taverne-license

Take down policy

If you believe that this document breaches copyright please contact us at:

repository@maastrichtuniversity.nl

providing details and we will investigate your claim.
}

Copyright and moral rights for the publications made accessible in the public portal are retained by the authors and/or other copyright owners and it is a condition of accessing publications that users recognise and abide by the legal requirements associated with these

- Users may download and print one copy of any publication from the public portal for the purpose of private study or research.

- You may not further distribute the material or use it for any profit-making activity or commercial gain

If the publication is distributed under the terms of Article $25 \mathrm{fa}$ of the Dutch Copyright Act, indicated by the "Taverne" license above, 


\section{Small fiber neuropathy and sodium channels \\ a paradigm shift}

Janneke G. J. Hoeijmakers 
(C) J.G.J. Hoeijmakers, 2014

Lay-out: Jos Hendrix

Cover: Gijs Hoeijmakers

Production: Datawyse/Universitaire Pers Maastricht ISBN 9789461592899

The studies described in this thesis were performed at the Department of Neurology of the Maastricht University Medical Center, Maastricht, the Netherlands and at the Center for Neuroscience and Regeneration Research, Veterans Affairs Medical Center, West Haven, CT 06516, USA

The printing of this thesis was financially supported by Pfizer. 


\section{Small fiber neuropathy and sodium channels \\ a paradigm shift}

\section{PROEFSCHRIFT}

ter verkrijging van de graad van doctor aan de Universiteit Maastricht, op gezag van de Rector Magnificus, Prof. dr. L.L.G. Soete volgens het besluit van het College van Decanen, in het openbaar te verdedigen op vrijdag 14 februari 2014 om 14.00 uur

door

Janneke Gertrude Jacobine Hoeijmakers 


\section{Promotiecommissie}

\section{Promotor:}

Prof. dr. R.J. van Oostenbrugge

\section{Copromotoren:}

Dr. C.G. Faber

Dr. I.S.J. Merkies

Beoordelingscommissie:

Prof. dr. M. Van Kleef, voorzitter

Prof. dr. C.E.M. De Die-Smulders

Prof. dr. I.N. van Schaik (Academisch Medisch Centrum, Amsterdam)

Prof. dr. H.J.M. Smeets

Dr. P.A.G. Volders 


\section{Contents}

$\begin{array}{ll}\text { Abbreviations } & 6\end{array}$

Chapter 1 General introduction and outline of thesis 9

Chapter 2 Small-fiber neuropathies: advances in diagnosis, 23 pathophysiology and management

Chapter 3 Gain-of-function $\mathrm{Na}_{\mathrm{v}} 1.7$ mutations in idiopathic small fiber neuropathy

Chapter 4 Intra- and interfamily phenotypic diversity in pain syndromes associated with a gain-of-function variant of $\mathrm{Na}_{\mathrm{v}} 1.7$

Chapter $5 \quad \mathrm{Na}_{\mathrm{v}}$ 1.7-related small fiber neuropathy:

impaired slow-inactivation and DRG neuron hyperexcitability

Chapter 6 Functional profiles of SCNgA variants in dorsal root ganglion 115 neurons and superior cervical ganglion neurons correlate with autonomic symptoms in small fiber neuropathy

Chapter 7 Small nerve fibers, small hands and small feet:

a new syndrome of pain, dysautonomia and acromesomelia in a kindred with a novel $\mathrm{Na}_{\mathrm{v}} 1.7$ mutation

Chapter 8 Genetic aspects of sodium channelopathy in small fiber neuropathy

Chapter 9 Summary, general discussion and future perspectives

Chapter 10 Nederlandse samenvatting

Dankwoord

Curriculum vitae

List of publications 


\section{Abbreviations}

\begin{tabular}{|c|c|}
\hline APs & Action potentials \\
\hline ARFS & Axon Reflex Flare Size \\
\hline CHEPs & Contact heat-evoked potentials \\
\hline CIP & Congenital insensitivity to pain \\
\hline DRG & Dorsal root ganglion \\
\hline FAP & Familial amyloid polyneuropathy \\
\hline GFP & Green fluorescent protein \\
\hline HIV & Human immunodeficiency virus \\
\hline IEM & Inherited erythromelalgia \\
\hline IENF & Intraepidermal nerve fiber \\
\hline IENFD & Intraepidermal nerve fiber density \\
\hline IES & Intraepidermal electrical stimulation \\
\hline IL & Interleukin \\
\hline I-SFN & Idiopathic small fiber neuropathy \\
\hline LEPs & Laser-evoked potentials \\
\hline $\mathrm{Na}_{\mathrm{v}} \mathrm{1} \cdot 3$ & Voltage-gated sodium channel 1.3 \\
\hline $\mathrm{Na}_{\mathrm{v}} 1.6$ & Voltage-gated sodium channel 1.6 \\
\hline $\mathrm{Na}_{\mathrm{v}} 1.7$ & Voltage-gated sodium channel 1.7 \\
\hline $\mathrm{Na}_{\mathrm{v}} 1.8$ & Voltage-gated sodium channel 1.8 \\
\hline $\mathrm{Na}_{\mathrm{v}} 1.9$ & Voltage-gated sodium channel 1.9 \\
\hline NCS & Nerve conduction studies \\
\hline NGF & Nerve growth factor \\
\hline NPS & Neuropathic Pain Scale \\
\hline PEPD & Paroxysmal extreme pain disorder \\
\hline PGP 9.5 & Protein gene product 9.5 \\
\hline PREPs & Pain-related evoked potentials \\
\hline QSART & Quantitative Sudomotor Axon Reflex Test \\
\hline QST & Quantitative sensory testing \\
\hline RMP & Resting membrane potential \\
\hline $\mathrm{SCN}_{3} \mathrm{~A}$ & Sodium channel, voltage-gated, type III, alpha subunit \\
\hline SCN8A & Sodium channel, voltage-gated, type VIII, alpha subunit \\
\hline $\mathrm{SCNgA}$ & Sodium channel, voltage-gated, type IX, alpha subunit \\
\hline SCN1OA & Sodium channel, voltage-gated, type $\mathrm{X}$, alpha subunit \\
\hline $\mathrm{SCN} 11 \mathrm{~A}$ & Sodium channel, voltage-gated, type XI, alpha subunit \\
\hline SFN & Small fiber neuropathy \\
\hline SFN-SIQ & SFN Symptom Inventory Questionnaire \\
\hline SGC & Superior cervical ganglion \\
\hline SSR & Sympathetic Skin Response \\
\hline SVR & Skin Vasomotor Reflex \\
\hline TNF-a & Tumor necrosis factor- $a$ \\
\hline TRP & Transient receptor potential \\
\hline TTX & Tetrodotoxin \\
\hline VAS & Visual Analogue Pain Scale \\
\hline VGSC & Voltage-gated sodium channel \\
\hline WT & Wild-type \\
\hline
\end{tabular}




Chapter 1

\section{General introduction and outline of thesis}




\section{Introduction}

\section{Small fiber neuropathy}

Small fiber neuropathy (SFN) is a condition characterized by neuropathic pain and autonomic symptoms, caused by dysfunction of the thinly myelinated Aס-fibers and unmyelinated C-fibers. As early as 1868, Paul Langerhans made drawings of the small diameter nerve fiber extensions in the human epidermis.' However, it took up until the last two decades, for SFN to be fully recognized as a distinct nosological entity. Despite debilitating symptoms, neurological examination and nerve conduction studies (NCS) are generally normal in the pure form of SFN. The introduction of specific diagnostic tests, such as quantitative sensory testing $(\mathrm{QST})^{2}$ and, in particular, intraepidermal nerve fiber density (IENFD) quantification by skin biopsy, ${ }^{3}$ made it possible to establish a more reliable diagnosis. 4,5

After the diagnosis SFN is made, an underlying cause has to be searched for, as some are potentially treatable. Diabetes mellitus is most frequently associated with $\mathrm{SFN}^{5}$, but also a causal relationship with HIV, hyperlipidaemia, amyloidosis, Fabry disease, celiac disease, sarcoidosis and other systemic illnesses is suggested..$^{6.8}$ However, the exact way in which these disorders results in the degeneration of the small nerve fibers is not elucidated yet. Besides, in a substantial proportion of patients diagnosed with SFN, ranging from $24 \%$ to $93 \%$ in different series, no underlying condition can be demonstrated.4, 7, 9 In this group of idiopathic SFN (I-SFN) patients causative treatment is impossible and therapy will mainly focus on neuropathic pain relief, unfortunately often with suboptimal effect. A better understanding of the pathophysiology of SFN is necessary to optimize patient management.

\section{Pathophysiology of peripheral neuropathies}

As with other peripheral neuropathies, multiple underlying conditions can cause SFN. The conditions that are associated with SFN can be classified into the following groups: metabolic, immune-mediated, toxic, infectious, hereditary and idiopathic. Many of these disorders also affect the large diameter nerve fibers, resulting in a mixed polyneuropathy. Several pathophysiological mechanisms have been proposed to cause peripheral neuropathies, usually related to the type of underlying condition. However, details about the degeneration of the small nerve fibers remain unknown.

The pathophysiology of diabetic polyneuropathy has been studied most, especially in rat models. Hyperglycaemia might disturb the polyol pathway, which through various changes may result in production of nerve toxic reactive oxygen..$^{10}$ Ischemia, in diabetes caused by vascular disease, may also induce reactive oxygen species. The smaller myelinated and unmyelinated fibers 
appear to be most sensitive for this." Nerve growth factor (NGF) would protect the nerve fibers against oxidative stress. In diabetes, however, impaired neurotrophic transport may lower the levels of NGF and could therefore indirect lead to nerve dysfunction. ${ }^{12}$ In addition, in two diabetes studies with capsaicin induced axotomy, a role for damaged axonal transport ${ }^{13}$ and impaired vascular regeneration ${ }^{14}$ was suggested.

Besides diabetic polyneuropathy, the pathophysiology of peripheral neuropathy in immunological disorders has also been studied extensively. In some small studies immunomodulatory therapy showed reduction of SFN-related symptoms, supporting the presumption of a causal relationship between an activated immune system and nerve fiber damage. . $^{19}$ In addition, serum autoantibodies, such as peripherin-lgG, anti-myelin-associated glycoprotein and antisulphatide antibodies have been described in patients diagnosed with SFN. ${ }^{20,21}$ Studies on pain in general suggested that both activation of the immune system at the peripheral level and the change in processing sensory information in the central nervous system have a role in pain associated with peripheral neuropathy. ${ }^{22,}{ }^{23}$ Furthermore, in SFN elevated levels of particular cytokines, such as tumor necrosis factor- $a$ (TNF- $\alpha$ ), interleukin (IL)- $1 \beta$ and IL- 6 have been demonstrated in the affected skin. ${ }^{24}$ Of these cytokines, TNF-a seems to play an important role as well in immune-mediated neuropathies such as Guillain-Barré syndrome, in which small nerve fibers are also involved. ${ }^{25,}{ }^{26}$ Elevated serum concentration of TNF-a shows a linear correlation with neuropathy severity in patients with Guillain-Barré syndrome and chronic inflammatory demyelinating polyradiculoneuropathy. ${ }^{27}, 28$

It is conceivable that the above-mentioned theories contribute to the development of SFN. However, it remains remarkable that such a great assortment of underlying conditions manifest in the $\sim$ same way, with a similar clinical pattern. Therefore, a final common pathophysiological pathway could serve as a plausible explanation.

A growing evidence for a genetic origin is rising. In burning feet syndrome, neuropathic pain of the lower extremities is the chief complaint. Family history research in this condition demonstrated an autosomal dominant inheritance, suggesting a genetic base for the small nerve fiber involvement. Though, the locus responsible for the neuropathy has not been revealed so far. 29,30

A suitable candidate gene for SFN would be the first step towards the unraveling of the pathophysiology. In this thesis the focus lies on the $S C N$ gA-gene, encoding the voltage-gated sodium channel (VGSC) $\mathrm{Na}_{\mathrm{v}}$ 1.7. The choice for studying this specific gene will be explained in the next paragraph. 


\section{Voltage gated sodium channels}

VGSCs play a fundamental role in the generation and conduction of action potentials in excitable cells, including the cells of the peripheral and central nervous system. ${ }^{31-33}$ The channels are integral membrane polypeptides that are formed by a large a-subunit, constructed of four homologous domains (DIDIV) with six transmembrane segments ( $\left.\mathrm{S}_{1}-\mathrm{S} 6\right)$, and one or more smaller auxiliary $\beta$-subunits. The $a$-subunit is the ion-selective pore-forming part and serves as a voltage sensor. The $\beta$-subunit provides the targeting and anchoring of the channels at specific sites in the plasma membrane. Besides, it modulates the gating properties of the a-subunit. ${ }^{3-34}$ In mammals, nine a-isoforms have been identified $\left(\mathrm{Na}_{\mathrm{v}} 1.1-\mathrm{Na}_{\mathrm{v}} \mathrm{1} .9\right)$, encoded by the SCN1A-SCN $5 A$ and SCN8A$S C N 11 A$ genes (Table 1). ${ }^{33} \mathrm{~A}$ distinction can be made on basis of the voltage dependence and kinetics, but also on the sensitivity to the sodium-channel blocker tetrodotoxin (TTX). Various human genetic disorders have been linked to mutations in these genes. ${ }^{33}$

Table 1. Classification sodium channels a-subunits

\begin{tabular}{|c|c|c|c|c|}
\hline Subtype & Gene & TTX & Localization & Human genetic disorders \\
\hline $\mathrm{Na}_{\mathrm{v}} 1.1$ & $\mathrm{SCN}_{1} \mathrm{~A}$ & $S$ & $\begin{array}{l}\text { CNS, cardiac myocytes, } \\
\text { DRG (large } \varnothing)\end{array}$ & $\begin{array}{c}\text { GEFS+, Dravet, SMEI, } \\
\text { familial hemiplegic migraine }\end{array}$ \\
\hline $\mathrm{Na}_{\mathrm{v}} 1.2$ & $\mathrm{SCN}_{2} \mathrm{~A}$ & $S$ & CNS & $\begin{array}{l}\text { Benign familial neonatal- } \\
\text { infantile seizures }\end{array}$ \\
\hline $\mathrm{Na}_{\mathrm{v}} 1 \cdot 3$ & $\mathrm{SCN}_{3} \mathrm{~A}$ & $S$ & $\begin{array}{l}\text { Embryonic DRG, SGN, } \\
\text { CNS, cardiac myocytes }\end{array}$ & $\begin{array}{l}\text { One case of epilepsy due to gain-of- } \\
\text { function mutation } 35\end{array}$ \\
\hline $\mathrm{Na}_{\mathrm{v}} 1.4$ & $\mathrm{SCN}_{4} \mathrm{~A}$ & $S$ & Skeletal muscle & $\begin{array}{c}\text { Periodic paralysis, paramyotonia } \\
\text { congenita, infantile-onset myasthenia }\end{array}$ \\
\hline $\mathrm{Na}_{\mathrm{v}} 1.5$ & $\mathrm{SCN}_{5} \mathrm{~A}$ & $\mathrm{IM}$ & $\begin{array}{l}\text { Embryonic DRG, } \\
\text { cardiac myocytes }\end{array}$ & $\begin{array}{l}\text { Long QT syndrome, Brugada } \\
\text { syndrome, progressive familial heart } \\
\text { block }\end{array}$ \\
\hline $\mathrm{Na}_{\mathrm{v}} 1.6$ & SCN8A & $S$ & $\begin{array}{l}\text { CNS, PNS, DRG } \\
\text { (all sizes } \varnothing)\end{array}$ & $\begin{array}{l}\text { One case of epilepsy due to gain of } \\
\text { function mutation }\end{array}$ \\
\hline $\mathrm{Na}_{\mathrm{v}} 1.7$ & $S C N g A$ & $S$ & $\begin{array}{c}\text { DRG (particularly small } \varnothing \text { ), } \\
\text { SGN, trigeminal ganglia }\end{array}$ & $\begin{array}{c}\text { Erythermalgia, paroxysmal extreme pain } \\
\text { disorder, insensitivity to pain }\end{array}$ \\
\hline $\mathrm{Na}_{\mathrm{v}} 1.8$ & SCN10A & $R$ & $\begin{array}{c}\text { DRG } \\
\text { (small and medium } \varnothing)\end{array}$ & Unknown \\
\hline $\mathrm{Na}_{\mathrm{v}} 1.9$ & SCN11A & $R$ & $\begin{array}{c}\text { DRG (small } \varnothing), \text { trigeminal } \\
\text { ganglia }\end{array}$ & Unknown \\
\hline
\end{tabular}

Legend to table 1. TTX = tetrodotoxin, $\mathrm{S}=$ sensitive, $\mathrm{R}=$ resistance, $\mathrm{IM}=$ intermediate sensitive , CNS = central nervous system, DRG = dorsal root ganglion, $\varnothing=$ diameter , SGN = sympathetic ganglion neuron PNS = peripheral nervous system, GEFS $+=$ generalised epilepsy with febrile seizures plus, $\mathrm{SMEI}=$ severe myoclonic epilepsy of infancy. 
In adults, $\mathrm{Na}_{\mathrm{v}} 1.6, \mathrm{Na}_{\mathrm{v}}$ 1.7, $\mathrm{Na}_{\mathrm{v}} 1.8$ and $\mathrm{Na}_{\mathrm{v}} 1.9$ are expressed in the small diameter DRG neurons that give rise to the A $\delta$-fibers and C-fibers, which are involved in SFN. Although $\mathrm{Na}_{\mathrm{v}} 1.3$ is only expressed in embryonic DRG, in adults an upregulation following axotomy has been seen. ${ }^{37}$

In human and animal studies, particularly $\mathrm{Na}_{\mathrm{v}}$ 1.7, $\mathrm{Na}_{\mathrm{v}} 1.8$ and $\mathrm{Na}_{\mathrm{v}}$ 1.9, have been identified as important for the signal transmission in the pathway of pain perception..$^{38-40}$ Dysregulated expression of the channels has been demonstrated in chronic pain, associated with trauma, inflammation and metabolic disorders, including diabetes mellitus. ${ }^{40-47}$

$\mathrm{Na}_{\mathrm{v}} 1.7$ that is encoded by the $\mathrm{SCN}_{\mathrm{gA}}$-gene is of particular interest, since mutations in this gene have been identified in three human pain disorders yet. In congenital insensitivity to pain (CIP), resistance for pain is the result of loss-of-function mutations. ${ }^{48}$ Opposite, gain-of-function mutations cause inherited erythromelalgia (IEM) and paroxysmal extreme pain disorder (PEPD). IEM is characterized by burning pain and a red discoloration of the extremities aggravated by warmth and exercise. ${ }^{49}$ The clinical picture of paroxysmal extreme pain disorder (PEPD) contains paroxysms of unbearable rectal, ocular and submaxillary pain associated with flushing of the buttocks, eyelids, periorbital skin and legs..$^{\circ}$

SFN shows some similarities with both disorders. The most obvious common symptom is neuropathic pain. As with IEM, in SFN the pain is mostly located in the distal extremities, ${ }^{51}$ however, a more patchy distribution, including facial pain as in PEPD, has also been described. ${ }^{52-54}$ Even though a decrease in small nerve fibers has been demonstrated in IEM as well, the prominent autonomic dysfunction in SFN, and its absence in IEM, is a clear distinguishing feature. ${ }^{55}$ Functional analysis by voltage clamp and current clamp methods in HEK293 cells and DRG neurons confirmed that $S C N 9 A$ mutations actually result in channel- and DRG dysfunction and the corresponding clinical picture. ${ }^{38,56-59}$ An understanding of the physiology is necessary to get insight into the effects of a mutation on channel function. A normal functioning voltage-gated sodium channel operates as follows (Figure 1): first, an electrical impulse, like a noxious stimulus in $\mathrm{Na}_{\mathrm{v}} 1.7$, activates the resting channel. Subsequently, the channel opens and facilitates sodium to flow into the cell, leading to depolarization of the cell membrane. This initiates an action potential and the perception of pain. In response to depolarization, fast inactivation occurs within 1-2 ms via an intracellular loop that connects the domains III and IV. This loop serves as an inactivation gate like a hinged lid. ${ }^{60}$ In addition to fast inactivation, the channel may also undergo slow inactivation, that appears to follow after prolonged or repetitive depolarization. It is speculated that this is related to $\mathrm{S}_{4}$ translocations which may rearrange the sodium channel pore. ${ }^{61}$ After inactivation, the channel has to refold. This recovering process is called repriming. During repriming the channel is refractory to additional stimulations. ${ }^{58}$ 
Figure 1. Physiology voltage gated sodium channel

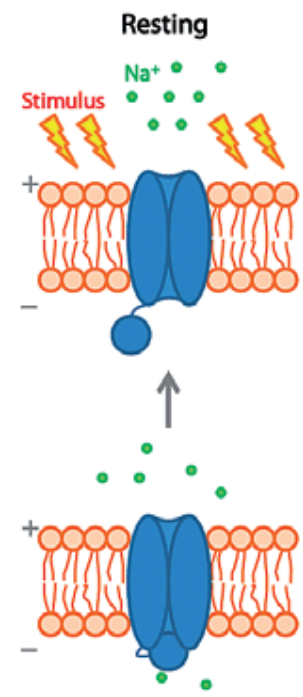

Closed
Open

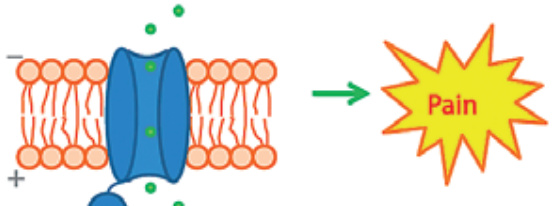

Repolarization

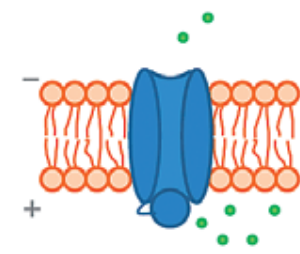

Inactivated

Legend to figure 1. Schematic representation of the changes in the a-subunit of $\mathrm{Na}_{\mathrm{v}} 1.7$ after stimulation by a noxious stimulus leading to the sensation of pain.

A stimulus results in opening of the channel, which facilitates sodium influx. This causes depolarization of the cell membrane, which results in an action potential. Via fast- and slow inactivation the channel closes and blocks the influx of sodium. Finally, the channel repolarizes and returns into the resting state.

Gain-of-function mutations can cause disturbances in different stages in this process, resulting in abnormal nerve cell firing and hyperexcitability of DRG neurons. In IEM and PEPD functional analyses have confirmed this, showing with specific features per condition which might be one of the explanations for the differences in clinical pattern. In IEM, $\mathrm{Na}_{\mathrm{v}}$.7 mutations showed hyperpolarized activation, slow deactivation and increase the ramp response of the channel.57, 62-65 In PEPD impaired fast inactivation has been demonstrated, leading to prolonged action potentials and repetitive neuron firing in response to provoking stimuli, such as stretching and cold temperatures..$^{50}$

In the main study of this thesis, the aim was to determine whether SCNgA mutations also play a role in the clinical picture and pathophysiology of SFN. To establish reliable results, a group of pure SFN patients, without signs of large nerve fiber involvement is necessary. In addition, no other associated conditions that might affect the nerve fibers in a specific way should be present. Because a 
'gold standard' for diagnosing SFN is not available yet, the combination of SFNrelated symptoms, a reduced IENFD in skin biopsy and abnormal temperature thresholds in QST, ensures the most certain diagnosis. In case mutations are found, functional analyses must be performed to provide information on pathogenicity.

\section{Differences in clinical pattern}

As already mentioned above, in SFN sensory symptoms generally manifest in a symmetrical length-dependent pattern, starting in the distal extremities with proximally extension. ${ }^{51}$ In contrast, to a lesser extent a non-length-dependent distribution can also occur, involving proximal regions of the limbs, face and trunk. It is suggested this pattern represents a ganglionopathy with selective involvement of the small DRG neurons. ${ }^{52-54}$ Burning mouth syndrome is a special example of a condition that might be the result of an isolated ganglionopathy of the trigeminal ganglion neurons. In these patients tongue biopsies showed a significantly lower IENFD than in controls. ${ }^{66}$

Sodium channels may play a role in the differences in clinical pattern in SFN. Besides the moment of disturbance in the process of channel activation and inactivation, also the cell type in which the mutant channel is expressed can be of interest. In fact, studies on VGSCs showed that channel mutations result in a neuron type-specific effect. ${ }^{67-69}$ For example, a study in IEM due to a $\mathrm{Na}_{\mathrm{v}}{ }^{1.7}$ mutation, showed that the mutant channel caused hyperexcitability in DRG and hypoexcitability in superior cervical ganglion (SCC) neurons, providing a molecular basis for the sympathetic autonomic symptoms. ${ }^{69}$ Related to this findings, it would be of interest to compare the effect on SGC function of a mutation that results in severe autonomic dysfunction with a mutation not leading to dysautonomic complaints.

Besides expression in DRG neurons and sympathetic ganglion neurons, $\mathrm{Na}_{\mathrm{v}} \mathrm{T} .7$ is also expressed in trigeminal ganglion neurons. This may be relevant in nonlength-dependent SFN. The $\mathrm{Na}_{\mathrm{v}} 1.7$ mutation $\mathrm{A}_{1} 632 \mathrm{E}$, which is associated with both characteristics of IEM and PEPD, showed that this mutation renders DRC and trigeminal ganglion neurons hyperexcitable. ${ }^{70} \mathrm{~A}$ role in burning mouth syndrome seems apparent. However, in a study of 7 patients with this syndrome no altered expression of $\mathrm{Na}_{\mathrm{v}} \mathrm{T} .7$ could be demonstrated. ${ }^{71}$

Therefore, in SCNgA mutations it may be useful to study the mutant channel within their native neuronal background, to understand the effects on the clinical picture. 


\section{General aim of this thesis}

The general aim of this thesis is to investigate the role of VGSC, and in particular $\mathrm{Na}_{\mathrm{v}} \mathrm{T} .7$, in the pathophysiology of SFN, in order to create better clinical understanding and future targeted therapeutic options.

\section{Outline of this thesis}

Chapter 2 provides a comprehensive review of the literature that has been published about SFN so far. Advances in diagnosis, pathophysiology and management are highlighted. New findings that are the result of the studies described in the next chapters are also addressed in this review.

Chapter 3 describes a clinically well-defined cohort of patients with biopsyconfirmed I-SFN in which the presence of SCNgA-gene mutations is demonstrated. In addition, functional analyses show the effects of the mutations on channel function and the DRG neuron firing properties.

Chapter 4 shows two siblings and one independent patient diagnosed with SFN, all housing the $\mathrm{Na}_{\mathrm{v}}$.7 variant $1228 \mathrm{M}$. The three patients presented with a remarkable clinical picture with facial or scalp pain. Therefore, the effect of the I228M mutation on the excitability of trigeminal ganglion neurons has been assessed.

The functional analysis of the $1739 \mathrm{~V}$ variant, demonstrated in one patient diagnosed with I-SFN in chapter 2, is highlighted in chapter 5 . The clinical findings are coupled to the electrophysiological results.

The degree of autonomic dysfunction may differ between patients diagnosed with SFN. To explain this difference in phenotype, in chapter 6 the functional profiles of two distinct $S \mathrm{SN}_{9} A$ variants in dorsal root ganglion neurons and superior cervical ganglion neurons are demonstrated, hereby validating the symptoms inventory questionnaire through biophysical findings.

During the assessment of patients for the above-mentioned study, one patient had notable anthropometric features of having an abnormal stature with small hands, lower legs and feet. His father and one brother showed similar features. All three turned out to carry a novel $\mathrm{Na}_{\mathrm{v}} 1.7$ mutation. In chapter 7 this new syndrome of pain, dysautonomia, small hands and small feet is described. 
Chapter 8 provides a review of the genetic aspects of sodium channelopathy in small fiber neuropathy following the results of the studies in this thesis. First, the role of $\mathrm{Na}_{\mathrm{v}} \mathrm{\gamma} .7$ in pain is discussed. In addition the molecular genetics and pathophysiology of all SCNgA-gene variants demonstrated in SFN are highlighted.

Finally, in chapter 9 the findings of this thesis are summarized. In addition, the current importance and future perspectives are stated. 


\section{References}

1. Langerhans, P. Uber die nerven der menschlichen haut. Virchows Archives of Pathological Anatomy 44, 325-337 (1868).

2. Yarnitsky, D. \& Sprecher, E. Thermal testing: normative data and repeatability for various test algorithms. J Neurol Sci 125, 39-45 (1994).

3. Wang, L., Hilliges, M., Jernberg, T., Wiegleb-Edstrom, D. \& Johansson, O. Protein gene product 9.5-immunoreactive nerve fibres and cells in human skin. Cell Tissue Res 261, 25-33 (1990).

4. Devigili, G. et al. The diagnostic criteria for small fibre neuropathy: from symptoms to neuropathology. Brain 131, 1912-25 (2008).

5. Tesfaye, S. et al. Diabetic neuropathies: update on definitions, diagnostic criteria, estimation of severity, and treatments. Diabetes Care 33, 2285-93 (2010).

6. Lauria, G. Small fibre neuropathies. Curr Opin Neurol 18, 591-7 (2005)

7. Lacomis, D. Small-fiber neuropathy. Muscle Nerve 26, 173-88 (2002).

8. Hoitsma, E. et al. Small fiber neuropathy: a common and important clinical disorder. J Neurol Sci 227, $119-30$ (2004).

9. Bednarik, J. et al. Etiology of small-fiber neuropathy. J Peripher Nerv Syst 14, 177-83 (2009).

10. Feldman, E.L. Oxidative stress and diabetic neuropathy: a new understanding of an old problem. J Clin Invest 111, 431-3 (2003).

11. Malik, R.A. et al. Hypoxic neuropathy: relevance to human diabetic neuropathy. Diabetologia $33,311-8$ (1990).

12. Anand, P. et al. The role of endogenous nerve growth factor in human diabetic neuropathy. Nat Med 2, 703-7 (1996).

13. Polydefkis, M. et al. The time course of epidermal nerve fibre regeneration: studies in normal controls and in people with diabetes, with and without neuropathy. Brain 127, 1606-15 (2004).

14. Ebenezer, G.J. et al. Impaired neurovascular repair in subjects with diabetes following experimental intracutaneous axotomy. Brain 134, 1853-63 (2011).

15. Hoitsma, E. et al. Improvement of small fiber neuropathy in a sarcoidosis patient after treatment with infliximab. Sarcoidosis Vasc Diffuse Lung Dis 23, 73-7 (2006).

16. Dabby, R., Gilad, R., Sadeh, M., Lampl, Y. \& Watemberg, N. Acute steroid responsive small-fiber sensory neuropathy: a new entity? J Peripher Nerv Syst 11, 47-52 (2006).

17. Souayah, N. et al. Effect of intravenous immunoglobulin on cerebellar ataxia and neuropathic pain associated with celiac disease. Eur J Neurol 15, 1300-3 (2008).

18. Wakasugi, D. et al. Extreme efficacy of intravenous immunoglobulin therapy for severe burning pain in a patient with small fiber neuropathy associated with primary Sjogren's syndrome. Mod Rheumatol 19 437-40 (2009).

19. Parambil, J.G., Tavee, J.O., Zhou, L., Pearson, K.S. \& Culver, D.A. Efficacy of intravenous immunoglobulin for small fiber neuropathy associated with sarcoidosis. Respir Med 105, 101-5 (2011).

20. Chamberlain, J.L. et al. Peripherin-IgG association with neurologic and endocrine autoimmunity. $J$ Autoimmun 34, 469-77 (2010).

21. Dabby, R., Weimer, L.H., Hays, A.P., Olarte, M. \& Latov, N. Antisulfatide antibodies in neuropathy: clinical and electrophysiologic correlates. Neurology 54, 1448-52 (2000).

22. Marchand, F., Perretti, M.\& McMahon, S.B. Role of the immune system in chronic pain. Nat Rev Neurosci 6, 521-32 (2005).

23. Pace, M.C. et al. Neurobiology of pain. J Cell Physiol 209, 8-12 (2006).

24. Uceyler, N. et al. Elevated proinflammatory cytokine expression in affected skin in small fiber neuropathy. Neurology 74, 1806-13 (2010).

25. Pan, C.L. et al. Cutaneous innervation in Guillain-Barre syndrome: pathology and clinical correlations. Brain 126, 386-97 (2003).

26. Martinez, V. et al. Small fibre impairment predicts neuropathic pain in Guillain-Barre syndrome. Pain 151, 53-60 (2010).

27. Creange, A., Belec, L., Clair, B., Raphael, J.C. \& Gherardi, R.K. Circulating tumor necrosis factor (TNF)alpha and soluble TNF-alpha receptors in patients with Guillain-Barre syndrome. J Neuroimmunol 68, 95-9 (1996). 
28. Misawa, S. et al. Serum levels of tumor necrosis factor-alpha in chronic inflammatory demyelinating polyneuropathy. Neurology 56, 666-9 (2001).

29. Stogbauer, F. et al. Autosomal dominant burning feet syndrome. J Neurol Neurosurg Psychiatry 67, 78-81 (1999).

30. Kuhlenbaumer, G. et al. A second family with autosomal dominant burning feet syndrome. Ann N Y Acad Sci 883, 445-8 (1999).

31. Waxman, S.G. The neuron as a dynamic electrogenic machine: modulation of sodium-channel expression as a basis for functional plasticity in neurons. Philos Trans R Soc Lond B Biol Sci 355, 199-213 (2000).

32. Lai, H.C. \& Jan, L.Y. The distribution and targeting of neuronal voltage-gated ion channels. Nat Rev Neurosci 7, 548-62 (2006).

33. Catterall, W.A., Goldin, A.L. \& Waxman, S.G. International Union of Pharmacology. XLVII. Nomenclature and structure-function relationships of voltage-gated sodium channels. Pharmacol Rev 57, 397-409 (2005).

34. Catterall, W.A. From ionic currents to molecular mechanisms: the structure and function of voltage-gated sodium channels. Neuron 26, 13-25 (2000)

35. Estacion, M., Gasser, A., Dib-Hajj, S.D. \& Waxman, S.G. A sodium channel mutation linked to epilepsy increases ramp and persistent current of $\mathrm{Nav1.3}$ and induces hyperexcitability in hippocampal neurons. Exp Neurol 224, 362-8 (2010).

36. Veeramah, K.R. et al. De novo pathogenic SCN8A mutation identified by whole-genome sequencing of a family quartet affected by infantile epileptic encephalopathy and SUDEP. Am J Hum Genet 90, 502-10 (2012).

37. Waxman, S.G., Kocsis, J.D. \& Black, J.A. Type III sodium channel mRNA is expressed in embryonic but not adult spinal sensory neurons, and is reexpressed following axotomy.J Neurophysiol 72, 466-70 (1994).

38. Dib-Hajj, S.D., Cummins, T.R., Black, J.A. \& Waxman, S.G. Sodium channels in normal and pathological pain. Annu Rev Neurosci 33, 325-47 (2010).

39. Waxman, S.G. Channelopathic pain: a growing but still small list of model disorders. Neuron $66,622-4$ (2010).

40. Lai, J., Porreca, F., Hunter, J.C. \& Gold, M.S. Voltage-gated sodium channels and hyperalgesia. Annu Rev Pharmacol Toxicol 44, 371-97 (2004).

41. Zhang, J.M., Donnelly, D.F., Song, X.J. \& Lamotte, R.H. Axotomy increases the excitability of dorsal root ganglion cells with unmyelinated axons. J Neurophysiol 78, 2790-4 (1997).

42. Wood, J.N., Boorman, J.P., Okuse, K. \& Baker, M.D. Voltage-gated sodium channels and pain pathways. J Neurobiol 61, 55-71 (2004).

43. Waxman, S.G. Transcriptional channelopathies: an emerging class of disorders. Nat Rev Neurosci 2, $652-9$ (2001).

44. Waxman, S.G. \& Hains, B.C. Fire and phantoms after spinal cord injury: $\mathrm{Na}+$ channels and central pain. Trends Neurosci 29, 207-15 (2006).

45. Kajander, K.C., Wakisaka, S. \& Bennett, G.J. Spontaneous discharge originates in the dorsal root ganglion at the onset of a painful peripheral neuropathy in the rat. Neurosci Lett 138, 225-8 (1992).

46. Wall, P.D. \& Devor, M. Sensory afferent impulses originate from dorsal root ganglia as well as from the periphery in normal and nerve injured rats. Pain 17, 321-39 (1983).

47. Craner, M.J., Klein, J.P., Renganathan, M., Black, J.A. \& Waxman, S.G. Changes of sodium channel expression in experimental painful diabetic neuropathy. Ann Neurol 52, 786-92 (2002).

48. Cox, J.J. et al. An SCNgA channelopathy causes congenital inability to experience pain. Nature $444,894-8$ (2006).

49. Yang, $\mathrm{Y}$. et al. Mutations in $\mathrm{SCN}_{9} \mathrm{~A}$, encoding a sodium channel alpha subunit, in patients with primary erythermalgia. J Med Genet 41, 171-4 (2004).

50. Fertleman, C.R. et al. SCNgA mutations in paroxysmal extreme pain disorder: allelic variants underlie distinct channel defects and phenotypes. Neuron 52, 767-74 (2006).

51. Gorson, K.C. \& Ropper, A.H. Idiopathic distal small fiber neuropathy. Acta Neurol Scand 92, 376-82 (1995).

52. Khan, S. \& Zhou, L. Characterization of non-length-dependent small-fiber sensory neuropathy. Muscle Nerve 45, 86-91 (2012).

53. Gorson, K.C. et al. Non-length dependent small fibre neuropathy/ganglionopathy. J Neurol Neurosurg Psychiatry 79, 163-9 (2008). 
54. Gemignani, F. et al. Non-length dependent small fiber neuropathy. a prospective case series. J Peripher Nerv Syst 15, 57-62 (2010).

55. Davis, M.D. et al. Histopathologic findings in primary erythromelalgia are nonspecific: special studies show a decrease in small nerve fiber density. J Am Acad Dermatol 55, 519-22 (2006).

56. Han, C. et al. Early- and late-onset inherited erythromelalgia: genotype-phenotype correlation. Brain $\mathbf{1 3 2}$ 1711-22 (2009).

57. Dib-Hajj, S.D. et al. Gain-of-function mutation in Nav1.7 in familial erythromelalgia induces bursting of sensory neurons. Brain 128, 1847-54 (2005).

58. Dib-Hajj, S.D. et al. Voltage-gated sodium channels in pain states: role in pathophysiology and targets for treatment. Brain Res Rev 60, 65-83 (2009).

59. Dib-Haji, S.D. et al. Transfection of rat or mouse neurons by biolistics or electroporation. Nat Protoc 4 1118-26 (2009).

6o. Vassilev, P.M., Scheuer, T. \& Catterall, W.A. Identification of an intracellular peptide segment involved in sodium channel inactivation. Science 241, 1658-61 (1988).

61. Vilin, Y.Y. \& Ruben, P.C. Slow inactivation in voltage-gated sodium channels: molecular substrates and contributions to channelopathies. Cell Biochem Biophys 35, 171-90 (2001).

62. Cummins, T.R., Dib-Hajj, S.D. \& Waxman, S.G. Electrophysiological properties of mutant Nav1.7 sodium channels in a painful inherited neuropathy.J Neurosci 24, 8232-6 (2004).

63. Han, C. et al. Sporadic onset of erythermalgia: a gain-of-function mutation in Nav1.7. Ann Neurol 59, 553-8 (2006).

64. Harty, T.P. et al. $\mathrm{Na}(\mathrm{V}) 1.7$ mutant $\mathrm{A} 863 \mathrm{P}$ in erythromelalgia: effects of altered activation and steady-state inactivation on excitability of nociceptive dorsal root ganglion neurons. J Neurosci 26, 12566-75 (2006).

65. Lampert, A., Dib-Hajj, S.D., Tyrrell, L. \& Waxman, S.G. Size matters: Erythromelalgia mutation S241T in Nav1.7 alters channel gating. J Biol Chem 281, 36029-35 (2006).66. Lauria, G. et al. Trigeminal small-fiber sensory neuropathy causes burning mouth syndrome. Pain 115, 332-7 (2005).

67. Cummins, T.R. et al. Nav1.3 sodium channels: rapid repriming and slow closed-state inactivation display quantitative differences after expression in a mammalian cell line and in spinal sensory neurons. $J$ Neurosci 21, 5952-61 (2001).

68. Choi, J.S., Dib-Hajj, S.D. \& Waxman, S.G. Differential slow inactivation and use-dependent inhibition of Nav1.8 channels contribute to distinct firing properties in IB4+ and IB4- DRG neurons. J Neurophysiol 97, $1258-65$ (2007).

69. Rush, A.M. et al. A single sodium channel mutation produces hyper- or hypoexcitability in different types of neurons. Proc Natl Acad Sci U S A 103, 8245-50 (2006).

70. Estacion, M. et al. NaV1.7 gain-of-function mutations as a continuum: A $632 \mathrm{E}$ displays physiological changes associated with erythromelalgia and paroxysmal extreme pain disorder mutations and produces symptoms of both disorders. J Neurosci 28, 11079-88 (2008).

71. Beneng, K., Renton, T., Yilmaz, Z., Yiangou, Y. \& Anand, P. Sodium channel Na v 1.7 immunoreactivity in painful human dental pulp and burning mouth syndrome. BMC Neurosci 11, 71 (2010). 



\section{Chapter 2}

\section{Small-fiber neuropathies:}

advances in diagnosis, pathophysiology and management

\section{J.G.J. Hoeijmakers', C.G. Faber ${ }^{a}$, G. Lauriab , I.S.J. Merkies ${ }^{a, c}$, S.G. Waxman ${ }^{d}$}

${ }^{\text {aDepartment }}$ of Neurology, Maastricht University Medical Center, Maastricht bNeuromuscular Diseases Unit, IRCCS Foundation, Carlo Besta, Milan, Italy 'Department of Neurology, Spaarne Hospital, Hoofddorp ${ }^{\mathrm{d} D e p a r t m e n t}$ of Neurology, Yale University School of Medicine, New Haven, CT, USA

Nature Reviews Neurology. 2012;8(7):369-79; Published with permission from the Nature Publishing Group 


\begin{abstract}
Small fiber neuropathy (SFN), a disorder of thinly myelinated $A \delta$-fibers and unmyelinated C-fibers, is clinically characterized by neuropathic pain symptoms and autonomic complaints. Diagnosis of SFN is challenging as the clinical picture can be difficult to interpret and results from nerve conduction studies are often normal. In cases of suspected SFN, measurement of intraepidermal nerve fiber density and/or analysis of quantitative sensory testing can enable diagnosis. New diagnostic techniques (including measurement of nerve fiber density using corneal confocal microscopy, and nociceptive evoked potentials) may contribute to the diagnostic work-up. SFN can be associated with systemic diseases such as immune-mediated disorders, but remains idiopathic in a substantial proportion of patients. Gain-of-function variants in the $\mathrm{Na}_{\mathrm{v}} 1.7$ sodium channel have recently been found in nearly $30 \%$ of patients with idiopathic SFN, but the mechanisms of axonal degeneration in the disorder remain under investigation. Identification of the systemic diseases underlying SFN will enable development of drugs that target affected pathways to improve the management of neuropathic pain and autonomic dysfunction. In this Review, we discuss recent advances in the diagnosis and pathophysiology of SFN, highlighting how improved understanding of these aspects of the disorder will contribute to better patient management.
\end{abstract}




\section{Introduction}

In an 1868 publication, Paul Langerhans made the first description of intraepidermal nerve fiber (IENF) endings.' The study included drawings depicting essentially the same architecture identified later using immunohistochemical staining of axonal cytoplasm with antibodies against protein gene product 9.5 (PGP 9.5) - a neuronal marker. Despite disagreement on Langerhans' work throughout the following decades, full recognition of his findings came in 1954 when Weddell and colleagues published a thorough review on nerve endings in mammalian skin, showing extensive epidermal innervation by fine, freely ending filament-like axons deriving from dermal stem fibers. ${ }^{2,3}$ In the mid 2oth century, the different types of peripheral nerve fibers captured the attention of researchers and physicians. ${ }^{4}$ In 1944, Erlanger and Gasser were awarded the Nobel Prize in Physiology or Medicine for their discovery of the highly differentiated functions of single nerve fibers. ${ }^{5}$ These investigators showed that the sense of touch is conveyed by large-diameter fibers, with temperature-encoding fibers being smaller, and fine pain-signaling fibers the finest. Subsequently, a classification of fibers into three groups (A, B and C), with subsections for the $A$ group, was proposed. The smallest-diameter fibers were classified as $A \delta$-fibers and C-fibers; ${ }^{5}$ these are the small-diameter extensions of the dorsal root ganglion (DRG) neurons. In the cutaneous layer, myelinated $A \delta$-fibers and unmyelinated C-fibers provide cold and warm sense, respectively, and relay the sensation of pain after their activation with noxious mechanical or thermal stimuli. Aठ-fibers have an additional role in preganglionic sympathetic and parasympathetic function, whereas C-fibers contribute to postganglionic autonomic functions. Exposure to noxious chemicals can also cause nerve activation in a subset of C-fibers. ${ }^{6}$

Small fiber neuropathy (SFN) is a condition that selectively involves $A \delta$-fibers and C-fibers. The clinical picture of the disorder is straightforward, with 'positive' and 'negative' sensory symptoms, and autonomic complaints. Pathologically, SFN is characterized by degeneration of distal terminations of small-diameter sensory fibers, observed as low IENF density (IENFD) on histological analysis of tissue from patients with the condition (Figure 1). Sometimes, the terms 'painful neuropathy' or 'autonomic neuropathy' are used as synonyms for SFN. However, neuropathic pain can also be a symptom of large-fiber neuropathy, and involvement of both small and large nerve fibers has been described in some autonomic neuropathies. ${ }^{8}$ Recently, variants in SCN9A, which encodes the $\mathrm{Na}_{\mathrm{v}} 1.7$ sodium channel, have been identified in patients diagnosed with idiopathic SFN. ${ }^{9}$ These mutations lead to abnormal spontaneous firing and enhanced evoked firing of DRG neurons, and are likely to induce axon degeneration through mechanisms not yet fully understood. 
Figure 1.

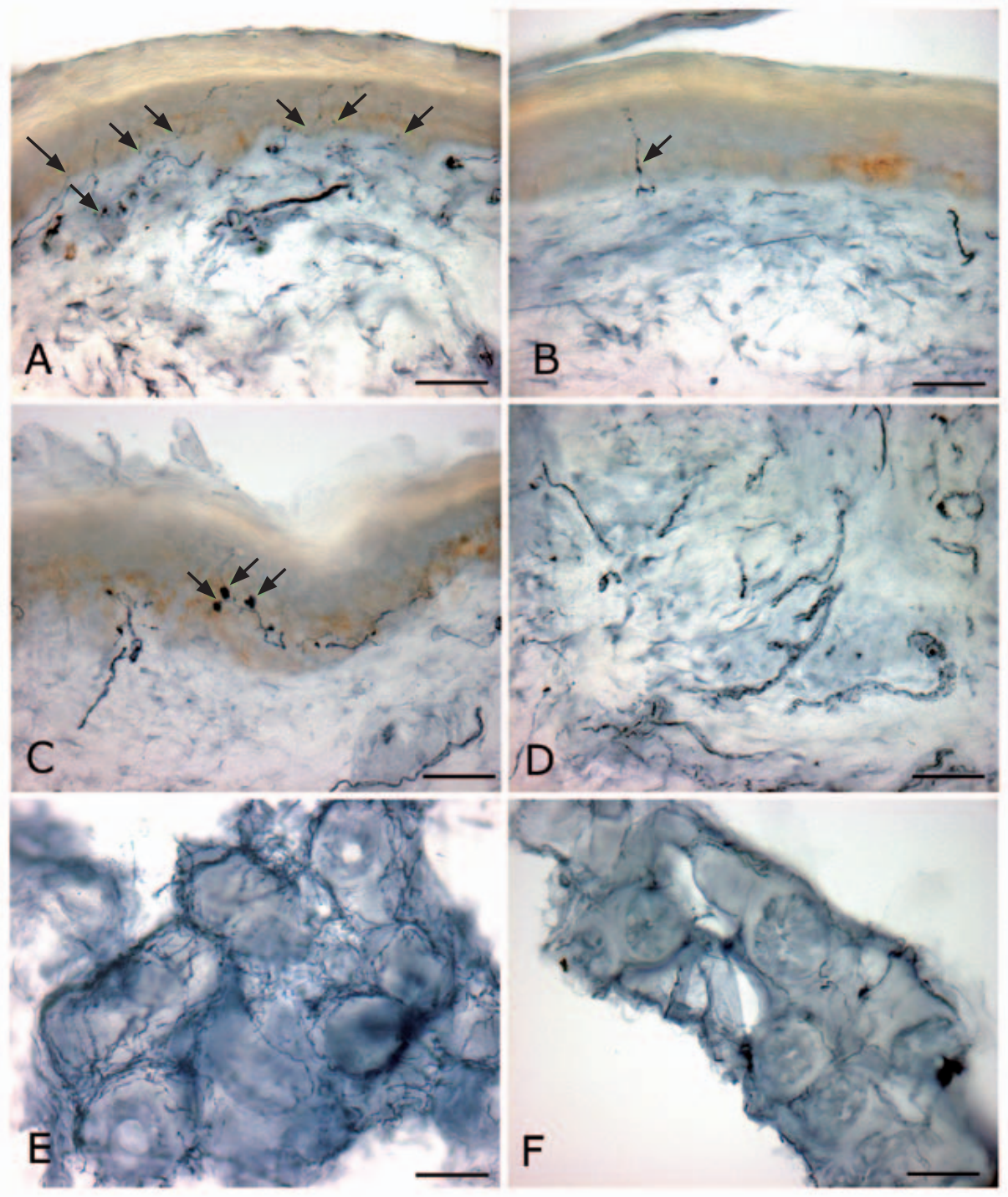

Legend to figure 1. Evidence of denervation in the skin of patients with SFN. Images show bright-field magnification of sections of skin biopsies from the distal part of the leg. Nerve fibers are stained with anti-PGP 9.5. A | Skin biopsy from a healthy individual, showing extensive intraepidermal nerve fibers (arrows). B | Staining of skin biopsy from a patient with SFN reveals severe depletion of intraepidermal nerve fibers (arrow) and dermal nerve bundles. C | Large swellings of intraepidermal nerve fibers (arrows) in a patient with SFN are considered to be predegenerative axonal changes. D | Fragmented dermal nerve fibers in a patient with SFN, which are weakly stained. E,F | Staining of sweat gland tissue shows extensive innervation in a healthy individual (e) and denervation in a patient with SFN ( $f$ ). Bar is $50 \mu \mathrm{m}$ in all images. Abbreviation: SFN, small-fiber neuropathy. 
Accurate diagnosis of SFN is important as it provides a basis for etiological work-up and treatment decisions. In this Review, we focus on advances in diagnosis, pathophysiology and management in SFN. Improvements in both the methods for diagnosis and understanding of the underlying mechanisms of disease will be essential to provide better patient care.

\section{Epidemiology}

The prevalence of SFN, either idiopathic or associated with systemic diseases, is unknown. Epidemiological information obtained from studies that focused on the diagnosis of SFN in selected subgroups of patients may have been biased owing to the selection criteria used. ${ }^{0,11}$ Furthermore, before the availability of diagnostic tools for SFN, diagnostic criteria for the disorder were not uniform. Consequently, patients with SFN were either undetected or misdiagnosed, thus limiting epidemiological analysis.

\section{Symptoms and clinical features}

Patients diagnosed with SFN experience multiple types of pain, described mainly as a burning sensation, shooting pains, prickling or itching. ${ }^{12}{ }^{13}$ Sheet or sock intolerance and restless legs syndrome may be present, often aggravating the foot pain sensation. Cramps and tingling may occur, mainly in the lower legs and feet, and are possibly induced following dysfunction of muscle nociceptors. ${ }^{12,14,15}$ Negative symptoms can include thermal sensory loss, loss of pinprick sensation, and numbness or tight feeling. ${ }^{12}$ Autonomic dysfunction may lead patients to complain of dry eyes or mouth, orthostatic dizziness, bowel disturbances (constipation, diarrhea, irritability, gastroparesis, cramps), micturation disturbances, changes in sweating (hyperhydrosis or hypohydrosis), accommodation problems, impotence, diminished ejaculation or lubrication, hot flushes, or cardiac palpitations (Box 1). ${ }^{14,16-18}$

Sensory symptoms in SFN usually occur in a symmetrical length-dependent pattern; that is, starting in the feet and expanding proximally. ${ }^{19}$ However, a non-length-dependent pattern of symptoms has been reported, showing a patchy distribution of neuropathy in the face, scalp, tongue or trunk., $13,20-22 \mathrm{~A}$ syringomyelia-like syndrome has been described in Tangier disease, ${ }^{23}$ which, similar to Fabry disease and familial amyloid polyneuropathy (FAP), is a multiorgan disease that can include SFN. ${ }^{24-26}$ FAP generally presents with SFN symptoms and signs, but rapidly evolves to a full mixed polyneuropathy. ${ }^{27}$ 
Box 1 | Symptoms of small-fiber neuropathy

For the diagnosis of small-fiber neuropathy, at least two of the following symptoms, not otherwise explained, are required.

\section{Sensory}

- Pain (burning, shooting, prickling or itching)

- Paraesthesias

- Allodynia

- Thermal sensory loss

- Pinprick loss

- Sheet or sock intolerance

- Restless legs syndrome

Autonomic

- Sicca syndrome

- Accommodation problems

- Hyperhydrosis or hypohydrosis

- Micturation disturbances

- Impotence and/or diminished ejaculation or lubrication

- Bowel disturbances (constipation, diarrhea, irritability, gastroparesis, cramps)

- Hot flushes

- Orthostatic dizziness

- Cardiac palpitations

\section{Diagnosis}

\section{Definition of SFN}

Many diagnostic definitions have been proposed for pure SFN. ${ }^{12,14,16,17,28}$ In general, the diagnosis should be considered in the presence of symptoms and/ or clinical signs of small-fiber damage (such as neuropathic pain, autonomic dysfunction, loss of pinprick sensation, thermal sensory loss, allodynia, or hyperalgesia) and in the absence of large-fiber involvement (such as muscle weakness, loss of light touch and/or proprioceptive or vibratory sensation, hypoflexia, or areflexia)..$^{10,11,14}$ In addition, nerve conduction studies, which are used to assess conduction in large-diameter fibers, should be normal. ${ }^{10-12,29} \mathrm{Skin}$ biopsy with quantification of IENFD and quantitative sensory testing (QST) are considered the cardinal additional tests that can be used to confirm the diagnosis of SFN. ${ }^{11}, 14$ 
In 2010, a practical diagnostic grading definition was proposed for diabetic SFN: possible SFN is defined as the presence of length-dependent symptoms and/ or clinical signs of small-fiber damage; probable SFN as the presence of lengthdependent symptoms, clinical signs of small-fiber damage, and normal sural nerve conduction studies; and a diagnosis of definite SFN is given if there are length-dependent symptoms, clinical signs of small-fiber damage, normal sural nerve conduction studies, altered IENFD at the ankle, and/or abnormal QST thermal thresholds at the foot. ${ }^{10}$ As damage of small nerve fibers is not diseasespecific, these diagnostic criteria can be applied to any patient with suspected SFN-including the idiopathic form-independently of the etiology. Although most cases of SFN present with length-dependent distribution of symptoms and signs, non-length-dependent types have been reported. 13,20

We have recently developed and validated the SFN Symptom Inventory Questionnaire (SFN-SIQ). ${ }^{28}$ This specific screening tool involves questioning the individual about 13 symptoms (Box 1 ) to which they can rate their experiences using a score of o-3: never, o; sometimes, 1; often, 2; or always, 3. A diagnosis of SFN is made in patients with at least two answers of score 1 or above, with evidence of IENFD and/or thermal threshold QST abnormalities, and after large-fiber impairment is ruled out. This tool has been used to screen patients with possible sarcoidosis-associated SFN or SCN9A mutations. ${ }^{9,28}$

No formal criteria for grading the severity of SFN exist, but simple, clinically orientated questionnaires, such as the SFN-SIQ, may be useful tools to determine the span and severity of SFN-related sensory and autonomic symptoms..$^{9,28}$

\section{Skin biopsy}

Skin biopsy is a minimally invasive, safe, largely painless and inexpensive investigative tool. Assessment of the biopsied tissue enables quantification of epidermal nerve endings - the distal ends of axons originating from DRC and trigeminal ganglia that cross the dermoepidermal junction and terminate within the epidermis. ${ }^{30}$ In 1983, an antibody against cytoplasmic PGP 9.5 was developed, and was shown to stain neurons better than did previously used antibodies, including those against neuropeptides, owing to the fact that antiPGP 9.5 stains not only epidermal but also dermal nerve fibers. ${ }^{31,32}$

\section{Punch biopsy}

For diagnostic purposes in cases of suspected SFN presenting with lengthdependent symptoms and signs, a skin biopsy can be taken from the distal part of the leg, within a region $10 \mathrm{~cm}$ above the lateral malleolus. ${ }^{33} \mathrm{~A}$ more proximal biopsy (for example, from the thigh) may be considered in patients with nonlength-dependent presentations, such as in suspected ganglionopathy. Notably, no normative values for PGP 9.5 staining are available for locations other than 
the distal leg. Bright-field immunohistochemistry or immunofluorescence, either with or without confocal microscopy, are used to assess IENF loss, ${ }^{32,34}$ but normative reference values adjusted for decade of life and sex are available only for immunohistochemical analysis..$^{35}$

IENFs are counted under the optical microscope and the number is divided by the length of the epidermal surface to obtain a linear density per millimeter; the density reported is the mean of the values calculated from at least three sections from the same biopsy. The diagnostic value of skin biopsy in patients with SFN has been established. ${ }^{33}$ In healthy individuals, IENFD in the distal leg is lower in males than in females, and the values decline with age. ${ }^{28,35-37}$ An IENFD below the fifth percentile is usually considered confirmatory for a diagnosis of SFN. In addition to IENF analysis, investigation of skin biopsies can reveal degenerative changes both in IENFs, such as progression to large axonal swellings (Figure 1C), and in dermal nerves, observed as fragmented and weak PGP 9.5 staining (Figure 1d). Moreover, skin biopsy can be used to investigate autonomic structures and innervation, such as in sweat glands (Figure 1d,e) and arrector pili muscles. Skin biopsy also provides the opportunity to quantify the innervation of dermal nerve fibers. ${ }^{38-40}$ IENFD is normal in about $12 \%$ of patients with symptoms of SFN," possibly representing pre-degenerative functional impairment of the nerve fibers. The combined assessment of IENFD and dermal nerve fibers can increase the sensitivity and specificity of skin biopsy for establishing a diagnosis of SFN. 39 In one study published in 2011, a reliable method for quantifying the innervation density of dermal nerves was presented; using this technique, the researchers found a correlation between dermal nerve morphometry and IENFD, and good interobserver agreement was demonstrated..$^{8}$

Skin biopsy has also been used in prospective studies to evaluate the course of SFN. For example, in toxic and hypothyroid neuropathies, skin biopsy analysis enabled researchers to conclude that reinnervation of the skin occurred after discontinuation of the toxic agent.41-43 In patients with Guillain-Barré syndrome, skin biopsy assessment was used to demonstrate that loss of IENFs began in the early phase of disease, and to reveal a correlation between nerve fiber loss and severity of neuropathic pain. ${ }^{44}$

Besides a role in establishing the diagnosis of SFN, skin biopsy can be used to aid in the identification of the underlying cause of the disorder. In some studies, attempts to define the diagnosis in patients with suspected SFN prompted an etiological work-up that revealed impaired glucose tolerance (as observed in $20-40 \%$ of patients) or other underlying systemic diseases." ${ }^{11,4}$. ${ }^{46}$ Skin biopsy assessment can reveal perivascular inflammation and vascular injury in cutaneous vasculitis resulting from systemic lupus erythematosus or eosinophilia. ${ }^{47}, 48 \mathrm{IgM}$ deposits on skin nerves were observed in patients with 
anti-myelin-associated glycoprotein neuropathy. ${ }^{49}$ Moreover, skin biopsy has been used to study the rate of degeneration and regeneration of IENFs after chemical-induced denervation with topical capsaicin or axotomy with punch biopsy, thus providing a method to investigate the potential therapeutic effects of neuroprotective drugs. ${ }^{50-55}$

Skin blister technique

An alternative method to obtain a skin biopsy is the skin blister technique..$^{56}$ Exertion of negative pressure on the skin leads to blistering, causing the epidermis to separate from the underlying dermis and enabling excision of the blister roof. IENFs in the tissue can be counted following immunostaining for neuronal markers. Skin blistering has benefits over skin punch biopsy: topical anesthesia is not needed and bleeding is reduced. IENFD determined from immunostained tissue obtained via the skin blister technique was found to correlate with values obtained using tissues from punch biopsy. ${ }^{57}$ Additional studies are required, however, to establish normative age-related and sex-related values and the reliability of this technique for use in the diagnosis of SFN.

\section{Quantitative sensory testing}

In the 1970s, the first automated systems to investigate different sensory modalities were developed. ${ }^{8,59}$ Since this time, routine assessment of thermal and vibration sensation in a quantitative manner has become possible. For the evaluation of small nerve fiber dysfunction, only temperature thresholds are measured. Usually, two types of testing - the method of levels and the method of limits-are used. ${ }^{60,61}$ Several studies have shown that QST may be useful in efforts to establish a diagnosis of SFN. ${ }^{11,62}$ However, this technique has some limitations. For reliable results, QST requires the patient to be alert and cooperative. Furthermore, a number of different QST instruments with different testing protocols, algorithms and normative values are available, ${ }^{63}$ and no consensus has been reached as to which method should be used as the standard approach. ${ }^{64,65}$ IENFD seems to be inversely correlated with both cold and warm thresholds on QST, but the correlation between nerve fiber density and specific sensory modalities is unclear."1,33 QST is not a specific test of peripheral nerve function; as such, CNS dysfunction owing to disorders such as stroke or multiple sclerosis may also produce QST abnormalities. For some organizations, QST has been considered more useful in population studies than as an aid for diagnosis in individual patients. ${ }^{64}$

\section{Corneal confocal microscopy}

Over the past decade, the noninvasive technique of in vivo confocal microscopy of the cornea has been developed, mainly for use in patients with diabetic 
neuropathy. Confocal microscopy in healthy individuals has confirmed that the cornea is innervated by both $A \delta$-fibers and C-fibers of trigeminal origin. ${ }^{66}$ The technique allows observation of the living eye in situ, at the cellular level. ${ }^{67} \mathrm{~A}$ correlation between low corneal nerve fiber density and severity of the somatic neuropathy and IENF loss in the distal leg has been described. ${ }^{68-71}$ Studies in the past few years have suggested that confocal microscopy might be useful in the diagnosis of idiopathic SFN, Fabry disease and immune-mediated SFN.71-74

\section{Nociceptive evoked potentials}

Selective activation of both $A \delta$-fibers and C-fibers is used in two types of nociceptive evoked potentials: laser-evoked potentials (LEPs) and contact heatevoked potentials (CHEPs). Induction of pain-related evoked potentials (PREPs) involves the preferential stimulation of $A \bar{\delta}$-fibers..$^{75} \mathrm{~A}$ relationship between poor nociceptive evoked potential response and severity of IENFD loss has been described. ${ }^{66-78}$ Intraepidermal electrical stimulation (IES) may also contribute to the detection of functional changes in peripheral fibers in SFN. ${ }^{79}$

\section{Laser-evoked potentials}

To obtain LEPs, the skin is stimulated with short radiant heat pulses that are emitted by a $\mathrm{CO}_{2}$ laser. Brain potential at the vertex can be subsequently recorded. Late LEPs reflect $A \delta$-fiber activation (200-400-ms latency range), and ultra-late LEPs reflect C-fiber activation (1000-ms latency range), ${ }^{80,81}$ with the amplitude of cerebral response correlating with the reported intensity of the perceived pain. ${ }^{82}$ An abnormal LEP can represent a disorder of the peripheral nerve, nerve plexus, nerve root, or spinal or brainstem nerves, and the technique seems to be diagnostically useful in SFN. ${ }^{83-85}$ However, laser stimulators are not widely available and the ultra-late LEPs are technically difficult to obtain. ${ }^{84}$

\section{Contact heat-evoked potentials}

Following the development of a heat-foil CHEP stimulator with extremely rapid heat rising time $\left(70{ }^{\circ} \mathrm{C} / \mathrm{s}\right)$, elicitation of pain and CHEPs can be achieved. ${ }^{86}$ Compared with LEPs, contact heat stimulators cause mechanical activation of the skin and stimulate a larger surface area, which makes missed stimulation of fibers less likely, even when only a few intact fibers remain. ${ }^{76}$ The stimulus of contact heat stimulators is natural and can be controlled very precisely. ${ }^{86,87}$ Furthermore, the technology is easy to use in the clinic, does not require eye protection, and has a low risk of causing skin irritation.77 Similar to LEPs, late $\mathrm{CHEPs}$ are associated with $\mathrm{A} \delta$-fiber activation, and ultra late $\mathrm{CHEPs}$ with $\mathrm{C}$-fiber activation. ${ }^{86,88}$ However, the diagnostic value of this method in SFN has not yet been systematically investigated, and normative values are lacking. 
Pain-related evoked potentials

Compared with LEPs and CHEPS, the technique to assess PREPs is less timeconsuming and easier to perform..$^{75}$ PREPs are obtained through the use of a concentric planar electrode that delivers electrical stimuli solely to the superficial layer of the dermis. The stimulus primarily depolarizes superficial nociceptive $A \delta$-fibers, thereby excluding the possibility of activation of deeper non-nociceptive fibers..$^{75}$ In patients with HIV-related SFN, a correlation between reduced IENFD and abnormal PREPs was found. ${ }^{78}$ Results from one study in patients with diabetes suggest that measurement of PREPs may contribute to early detection of SFN, although this study did not include assessment of IENFD by skin biopsy. ${ }^{89}$

\section{Intraepidermal electrical stimulation}

For IES, a pushpin-like electrode of $0.2 \mathrm{~mm}$ in length is gently pressed against the skin, inserting the needle tip adjacent to the thin nerve endings in the skin. After delivery of an electrical stimulus, the evoked potential is measured in the same way as in the other nociceptive evoked potentials, and leads to preferential activation of $A \delta$-fibers. ${ }^{79}$ The utility of IES for the detection of SFN remains to be validated.

\section{Microneurography}

Microneurography has made recording of single $A \delta$-fiber and C-fiber activity possible, and provides a direct method for measuring sympathetic activity. This technique has contributed substantially to improving our knowledge of the physiology of nociceptors and the mechanisms underlying their sensitization. ${ }^{90-93}$ However, the application of microneurography requires both an expert investigator and a collaborative patient, and is invasive and timeconsuming, thus limiting the routine use of this technique in the clinical setting..$^{82,94}$

\section{Autonomic testing}

Autonomic involvement in SFN can be difficult to demonstrate. Cutaneous innervation of the dermal autonomic adnexa (such as sweat glands, hair follicles, blood vessels and pilomotor muscles) has been studied by staining biopsies from these tissues with antibodies against PGP 9.5 or vasoactive intestinal peptide and dopamine $\beta$-hydroxylase. ${ }^{95-97}$ Several tests to assess autonomic dysfunction also exist.

The Ewing battery for cardiovascular autonomic reflex testing is easy to perform, but has low sensitivity for the detection of autonomic dysfunction in patients with SFN. ${ }^{16,62,98}$ Sudomotor and vasodilator function tests include the Quantitative Sudomotor Axon Reflex Test (QSART), Thermoregulatory Sweat 
Test, Sympathetic Skin Response (SSR), Skin Vasomotor Reflex (SVR), and Axon Reflex Flare Size (ARFS). ${ }^{82,94,99}$ QSART seems to be sensitive in SFN, but as the application of this test requires specific skills and instrumentation, it is only available in specialized centers worldwide. ${ }^{16,98-100}$ By contrast, SSR and SVR are simple methods and can be used in any clinical neurophysiology laboratory, although their diagnostic value in SFN is reported to be poor. ${ }^{62,82,99}$ In one study, use of both SSR and SVR in combination with microneurography enabled differentiation between patients with autonomic SFN, those without autonomic complaints, and controls. ${ }^{101}$

The ARFS is a noninvasive method, the results of which seem to correlate with IENFD. ${ }^{102}$ This technique is used to measure the size of axon-reflex flare following electrical stimulation that simultaneously activates axon reflexes of sudomotor fibers and nociceptors. This mode of stimulation causes the release of vasodilating calcitonin gene-related peptide from C-fiber endings and acetylcholine from sympathetic nerve endings. Scanning laser Doppler flowmetry enables the measurement of skin blood flow in perfusion units, which represent the product of velocity and concentration of the blood cells moving within the volume measured. Using thermostatic laser Doppler probes that include both recording and heating elements, the underlying skin area is heated while blood perfusion is recorded. This examination can be performed in the area from where a skin biopsy is obtained, thus allowing direct correlation with findings from histological analysis. The parameters analyzed using scanning laser Doppler flowmetry are basal cutaneous blood flow, vasoconstriction reflexes induced by deep breathing and postural variation (venoarteriolar reflex), and vasodilatation induced by local heating (from $32{ }^{\circ} \mathrm{C}$ to $44{ }^{\circ} \mathrm{C}$ for $6 \mathrm{~min}$ ). Vasoconstriction reflexes induced by deep breathing can be used to examine sympathetic adrenergic function, whereas venoarteriolar reflex (caused by postural variation) and vasodilatation induced by local heating are used to investigate skin axonal reflexes carried by somatic C-fibers. By using scanning laser Doppler flowmetry, researchers measured the size of the flare caused by the vasodilation and showed that ARFS is reduced in patients with SFN. ${ }^{102,103}$

Two new techniques have been recently introduced to quantify the innervation density of sweat glands ${ }^{96}$ and arrector pili muscles, ${ }^{97}$ and these tools could widen the diagnostic yield of skin biopsy in diabetic SFN. Sweat gland nerve fiber density was initially quantified by manual morphometry after staining biopsied tissue with anti-PGP 9.5 antibodies. ${ }^{96}$ In the past few years, a new computerized area-based morphometric technique has been developed that involves counterstaining of the nerve fibers with Congo red. This technique has reduced variation in sweat gland area measurement compared with the manual method. ${ }^{104}$ In diabetic neuropathy, the sweat gland innervation index obtained using this new technique was found to correlate with glycaemic 
control and autonomic symptoms. ${ }^{105} \mathrm{~A}$ novel technique to quantify pilomotor nerves also showed good discriminative and reliability values in patients with diabetic neuropathy. ${ }^{97}$ Although both techniques seem very promising and may complement the investigation of IENFD for diagnosis of SFN, normative agematched and sex-matched values are needed for both tools before they can be implemented for general clinical use.

\section{Causes}

After an SFN diagnosis is made, the etiology must be investigated to detect underlying causes, as some are potentially treatable (Box 2). ${ }^{14,17,18}$ Diabetes mellitus, HIV, hyperlipidaemia, amyloidosis, Fabry disease, celiac disease, sarcoidosis and other systemic illnesses can all cause SFN. ${ }^{10,21,27,28,45,46,106-112}$ Among the hereditary sensory and autonomic neuropathies, types I, IV and V are characterized by predominant small nerve fiber involvement. ${ }^{24},{ }^{113}$ Despite a comprehensive work-up of patients with SFN, the proportion of individuals diagnosed with idiopathic or cryptogenic forms remains substantial, ranging from $24 \%$ to $93 \%$ in different series depending on the definition of SFN used."1, 17,114 In our cohort, no underlying cause was found in approximately one-third of patients. ${ }^{9}$ Some disorders cause pure SFN, whereas others cause SFN that may evolve to a mixed (small-fiber and large-fiber) neuropathy (Box 2).

Diabetes mellitus is the disease most frequently associated with SFN. About $50 \%$ of all patients with diabetes will develop neuropathy that generally involves both large and small nerve fibers., 10 Nerve dysfunction may also be seen in patients with impaired glucose tolerance, ${ }^{45}, 115$ a condition that predisposes individuals to diabetes. A glucose tolerance test should, therefore, be part of the routine work up in patients with suspected SFN.

Reduced IENFD has been described in patients with neurodegenerative disorders such as amyotrophic lateral sclerosis and Parkinson disease, and could contribute to the autonomic complaints in individuals with these conditions. ${ }^{116,177}$ A reduced IENFD has also been described in individuals with Kennedy disease; however, this finding is not surprising as the disorder typically causes sural nerve conduction abnormalities, a change that indicates large sensory fiber damage. ${ }^{118}$

The symptoms of some unexplained pain syndromes might also be the result of small nerve fiber dysfunction; for example, complex regional pain syndrome type I was linked to focal SFN in one study. ${ }^{119}$ Burning mouth syndrome has been described as a trigeminal SFN, a contention supported by evidence of low nerve fiber density in tongue biopsies from subgroups of patients. ${ }^{120,121}$ 
Box 2 | Conditions associated small fiber neuropathies

Pure and predominantly somatic small-fiber neuropathy

Metabolic

Impaired glucose tolerance, ${ }^{45,46}$ hyperlipidaemia, ${ }^{110}$ hypothyroidism ${ }^{147}$ Immune-mediated

Sarcoidosis, "' Sjögren syndrome, ${ }^{148}$ celiac disease, ${ }^{21}$ inflammatory bowel diseases, ${ }^{112}$ paraneoplastic neuropathy ${ }^{149}$

Infectious

Leprosy, ${ }^{150}$ Epstein-Barr virus ${ }^{151}$

Toxic and drugs

Antiretroviral drugs, bortezomib, metronidazole, ${ }^{152,153}$ flecainide, ${ }^{154}$ nitrofurantoin, ${ }^{155}$ alcohol abuse ${ }^{109,136}$

Hereditary

$\mathrm{Na}_{\mathrm{v}}{ }^{1.7-m u t a t i o n s},{ }^{9}$ Fabry disease, ${ }^{108}$ erythromelalgia, ${ }^{140}$ Ross syndrome, ${ }^{156}$ haemochromatosis ${ }^{157}$

Idiopathic

Idiopathic small-fiber neuropathy, ${ }^{19}$ burning mouth syndrome ${ }^{120}$

\section{Small-fiber neuropathy progressing to mixed-fiber neuropathy}

Metabolic

Diabetes, ${ }^{10}$ chronic kidney disease ${ }^{158}$

Immune-mediated

Amyloidosis, ${ }^{133}$ vasculitis, ${ }^{106}$ systemic lupus erythematodes, ${ }^{47}$ Guillain-

Barré syndrome ${ }^{159}$

Infectious

$\mathrm{HIV},{ }^{107}$ hepatitis $\mathrm{C}^{160}$ Lyme neuroborreliosis $^{161}$

Toxic and drugs

Hypervitaminosis B6 ${ }^{162}$

Hereditary

Familial amyloidosis, ${ }^{27}$ Fabry disease, ${ }^{108}$ Tangier disease,${ }^{23}$ Friedreich ataxia, ${ }^{163}$ cerebrotendinous xanthomatosis, ${ }^{164}$ hereditary sensory autonomic neuropathies ${ }^{113}$

Disorders with reduced intraepidermal nerve fiber density (no clear small-fiber neuropathy)

Neurodegenerative

Spinobulbar muscular atrophy (Kennedy disease), ${ }_{118}^{18}$ amyotrophic lateral sclerosis, ${ }^{116}$ Parkinson disease ${ }^{117}$

Idiopathic

Complex regional pain syndrome type l'19 


\section{Pathophysiology}

Several pathophysiological mechanisms have been proposed to cause peripheral neuropathy. However, details of the pathogenesis in these disorders are incompletely understood, and much remains to be learned about the pathophysiology of isolated SFN. Some pathophysiological mechanisms have been identified in mixed polyneuropathies, and these mechanisms may also have a role in SFN.

\section{Diabetes and other metabolic disorders}

Several hypotheses for neuropathic dysfunction in diabetic neuropathy have been suggested, with most evidence obtained from studies in rat models of the disease. One suggestion is that hyperglycemia might disturb the polyol pathway, which would result in an increased level of sorbitol. This and other changes in the pathway may lead to production of reactive oxygen species that can cause nerve damage. ${ }^{122}$

Evidence suggests that vascular disease in patients with diabetes can lead to ischemia, creating oxidative stress and resulting in the production of noxious reactive oxygen species. In a rat model of induced nerve infarction (which leads to ischemia), neuropathy preferentially affected smaller myelinated and unmyelinated fibers. ${ }^{123}$ Furthermore, hypoxia without ischaemia has been suggested as the underlying cause of degeneration in unmyelinated axons in patients with chronic obstructive airways disease. ${ }^{124}$

Impaired neurotrophic transport has been suggested as a pathophysiological mechanism that contributes to the development of SFN in diabetes. ${ }^{125}$ Decreased proliferation of keratinocytes in the skin of individuals with diabetes may lead to low levels of nerve growth factor (NGF) - a cytokine that is necessary for the survival of sympathetic fibers and for maintenance of phenotypic properties of small-diameter sensory fibers. In addition, NGF is reported to increase the resistance of the nerve cell to injury from oxidative stress. ${ }^{225}$ As such, lack of NGF could lead to nerve dysfunction.

Results from studies of induced denervation following topical capsaicin application have suggested an impairment of axon trafficking in diabetes, with reduced nerve fiber regeneration demonstrated in individuals with diabetes compared with healthy controls..$^{51}$ Other findings suggest that impaired vascular regeneration may have a role in diabetic neuropathy. 53 In light of the observation that nerve cells in patients with end-stage kidney disease were in a chronically depolarized state, one study suggested that hyperkalaemia may be a major contributor to nerve damage in this condition. ${ }^{226}$ 


\section{Immune-mediated SFN}

Several immune-mediated diseases may cause SFN (Box 2); in these instances, contribution of the immune system to small nerve fiber degeneration seems likely. Activation of the immune system stimulates mast cells and macrophages, which can lead to recruitment of neutrophils and monocytes that release chemokines and other mediators such as tumour necrosis factor-a (TNF-a), IL-1 $\beta$, IL- 6 and/or nitric oxide-cytokines involved in microglial cell activation. Evidence suggests that both the activation of the immune system at a peripheral level and the change in processing of sensory information in the CNS have a role in pain associated with peripheral neuropathy. ${ }^{127,128}$ In patients with lengthdependent SFN, higher levels of the cytokines TNF- $\alpha$, IL-1 $\beta$ and IL-8 were found in the affected distal skin than in the non-affected skin. Moreover, IL-6 and IL-8 expression was increased in patients with SFN compared with healthy individuals. Collectively, this evidence supports the idea that elevated local proinflammatory cytokines may be involved in the pathophysiology of pain in SFN. ${ }^{29}$

Another indication of immunological involvement in SFN is the presence of serum autoantibodies, such as peripherin-lgG, anti-myelin-associated glycoprotein and antisulphatide antibodies, which have all been reported in patients with SFN. ${ }^{130,131}$

Although large-diameter nerve fibers are considered to be the main target of immunological attacks in chronic inflammatory demyelinating polyneuropathy, small nerve fiber reduction has also been demonstrated. ${ }^{132}$ Amyloid neuropathy predominantly involves small nerve fibers, although large-fiber dysfunction is often found. In amyloidosis, nerve fiber degeneration may be a result of mechanical compression by amyloid deposits. ${ }^{133}$ Compression of DRG, plexus and distal trunks could explain distal axonal degeneration of nerve fibers, with longer fibers more likely to be damaged on a probabilistic basis. ${ }^{134}$ However, toxic or metabolic factors might also have a role in amyloidosisassociated neuropathy. ${ }^{133}$ Cutaneous vasculitis, as observed in systemic lupus erythematosus and eosinophilia-associated neuropathy, can also be associated with SFN; in this context, the pathogenesis includes vasculitic injury and eosinophilic neurotoxicity. 47,48

\section{Alcohol abuse}

Axonal degeneration in SFN related to alcohol abuse has been attributed to the direct toxic effect of ethanol on nerve fibers, contrary to the previously suggested hypothesis that nutritional deficiency might be the main cause. ${ }^{109,}, 135,136$ Ethanol can cause metabolic disturbances with inhibition of axonal transport; ;35 however, alcohol abuse usually causes a mixed axonal neuropathy. 


\section{Ion channel dysfunction}

Channelopathies have recently been linked to multiple pain syndromes. ${ }^{137}$ Different members of the transient receptor potential (TRP) family of cation channels have been studied in animal models. Studies in a rat model of paclitaxel-induced painful peripheral neuropathy have demonstrated a role for the cation channel subfamily member Trpv $4 .{ }^{138}$ Blockade of the ion channel Trpa 1 in diabetic rats was reported to reduce loss of cutaneous nerve fiber function. ${ }^{39}$ A recent study has demonstrated the presence of single amino acid substitutions of the voltage-gated sodium channel $\mathrm{Na}_{\mathrm{v}} 1.7$-which is preferentially expressed within DRG and sympathetic ganglion neurons-in a substantial fraction of patients with idiopathic SFN. ${ }^{9}$ A total of $28.6 \%$ of patients with idiopathic SFN were found to carry a gain-of-function variant in $\mathrm{Na}_{\mathrm{v}}$ 1.7. The variants, each affecting a single amino acid residue along the $\mathrm{Na}_{\mathrm{v}} \mathrm{\gamma} .7$ channel protein backbone (Figure 2), produced a variety of pro-excitatory changes in channel function, impairing various forms of channel inactivation and enhancing resurgent current production by the channels. When introduced into cultured DRG neurons, the mutations caused inappropriate spontaneous firing and enhanced responsiveness to depolarizing stimuli; this effect is proposed to cause small nerve fibers to degenerate.

$\mathrm{Na}_{\mathrm{v}} 1.7$ mutations have also been linked to inherited erythromelalgia (IEM) and paroxysmal extreme pain disorder-two diseases that show some clinical similarities with SFN. ${ }^{9}, 140$ Both IEM and SFN can present with distal burning pain. IEM is defined by the presence of a red discoloration of the extremities that tends to be triggered by warmth and exercise. ${ }^{140}$ Skin discoloration is seen in one-third to two-thirds of patients with SFN. Moreover, most patients with IEM

Figure 2.

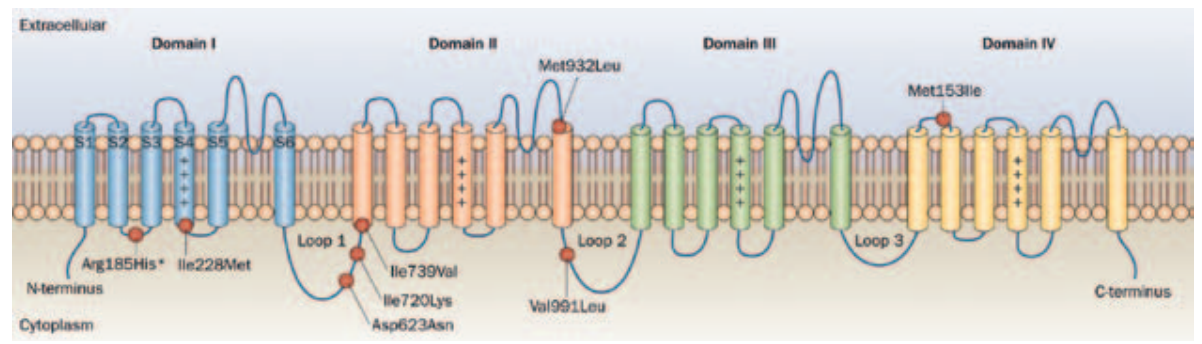

Legend to figure 2. SFN-associated variants in the $\mathrm{Na}_{\mathrm{y}} 1.7$ sodium channel. Schematic representation of the $\mathrm{Na}_{\mathrm{v}} 1.7$ sodium channel showing the locations of the variants found in patients with idiopathic SFN. *Two patients had an Arg185 His substitution. Abbreviation: SFN, small-fiber neuropathy. Modified from Faber, C. G. et al. Gain of function $\mathrm{Na}_{\mathrm{v}} \mathrm{T} .7$ mutations in idiopathic small fiber neuropathy. Ann. Neurol. 71, 26-39 (2012) (c) John Wiley and Sons. 
describe relief with cooling, a feature that is seen in some but not all patients with SFN. Importantly, the autonomic complaints that are characteristic of SFN (Box 1 ) and the myalgias seen in some patients with SFN are not-or are only rarely-observed in IEM. Interestingly, SFN has been reported in some patients with erythromelalgia, ${ }^{141}$ and the relationship between IEM and SFN is under investigation.

\section{Management}

When an underlying condition has been demonstrated, causative treatment should be instituted if possible. For idiopathic SFN, treatments can be administered to control the symptoms of pain. However, the effects of treatment on the course of SFN have thus far not been studied for most conditions involving SFN.

\section{Diabetic neuropathy}

In diabetic neuropathy, tight glycaemic control seems to contribute to prevention of neuropathy and even to amelioration of symptoms..$^{10}$ As evidence has linked diabetic neuropathy to hypertension, smoking, obesity, elevated triglyceride levels, and the presence of cardiovascular disease, treatment of these potentially modifiable cardiovascular risk factors might influence the course of the neuropathy. ${ }^{15}$

\section{Fabry disease}

Replacement of a-galactosidase A-the enzyme that is lacking in patients with Fabry disease- has been shown to reduce neuropathic pain, restore the sweating reflex, and reduce the detection threshold for cold and warm temperatures in the hands and feet in patients with the disorder. ${ }^{142}$ However, no epidermal nerve fiber regeneration has been demonstrated. ${ }^{143}$

\section{Immune-mediated neuropathy}

In immune-mediated conditions, some case studies of immunomodulatory therapy seem to show efficacy of this approach in relieving SFN symptoms. ${ }^{144}$ ${ }^{145}$ However, large, prospective, controlled studies are needed to confirm these results.

\section{Idiopathic SFN}

For patients with idiopathic SFN, treatments can generally target only symptomatic outcomes with the aim of ameliorating neuropathic pain. Tricyclic antidepressants and serotonin-norepinephrine reuptake inhibitors 
are recommended as first-line treatment for neuropathic pain. ${ }^{146}$ Opioids can be used to treat exacerbations of pain, and efficacy of lamotrigine has been demonstrated in HIV-associated polyneuropathy. ${ }^{146}$ Unfortunately, the available drugs for SFN often provide only partial relief from pain. More insight into the pathophysiology of neuropathic pain could contribute to better management of pain and, perhaps, to the development of more-effective pharmacotherapies. Selective $\mathrm{Na}_{\mathrm{v}} \mathrm{T} .7$ blockers are currently under development, and might prove effective, especially in patients with $S C N g A$ mutations. ${ }^{9}$

\section{Conclusions}

As diagnostic methods have improved and awareness of SFN within the clinical community has increased, SFN has become more widely recognized in clinical practice. Understanding of the pathophysiology of this disorder has also improved. In the absence of a gold-standard diagnostic test, the SFN diagnosis is established by the presence of typical SFN-related symptoms, normal nerve conduction studies, reduced IENFD at the ankle, and/or abnormal QST. New techniques, such as determination of dermal nerve fiber density in skin biopsy, nociceptive evoked potentials, and corneal confocal microscopy, are emerging. The capacity of these techniques to aid in the diagnosis of SFN will, hopefully, soon be determined.

Several potentially treatable conditions have been linked to SFN and should be the focus of an etiological work-up in patients with this condition. The pathophysiology of SFN, although still incompletely understood, is the focus of current studies. Prospective controlled studies are needed to investigate the effects of immunomodulatory therapy and other approaches, including subtypeselective sodium channel blockade in SFN associated with $\mathrm{Na}_{\mathrm{v}} \mathrm{T}$.7 mutations. Results of these studies will almost certainly enhance our understanding of $\mathrm{SFN}$, and may reveal new avenues for therapy.

\section{Key points}

- Small-fiber neuropathy (SFN) is a disorder of thinly myelinated $A \bar{\delta}$-fibers and unmyelinated C-fibers

- SFN is diagnosed on the basis of presence of typical SFN-related symptoms, normal nerve conduction studies, reduced intraepidermal nerve fiber density at the ankle, and/or abnormal quantitative sensory testing

- SFN can be associated with systemic diseases, with an immune-mediated basis proposed in some cases; however, the cause remains unclear in a substantial number of patients 
- Mutations in $\mathrm{SCN}_{9} \mathrm{~A}$, which encodes the sodium channel $\mathrm{Na}_{\mathrm{v}}{ }^{1} .7$, were found to underlie SFN in a subset of patients

- Therapy for SFN focuses mainly on pain relief, management of autonomic dysfunction, and disease modification where possible

- Future studies into therapies for SFN should address the efficacy of immunomodulating agents and selective sodium channel blockers

\section{Review criteria}

A literature search was performed to find studies and reviews published on small-fiber neuropathy (SFN). If appropriate, historical papers were also included. A PubMed search was performed using the keywords "small fiber (fibre) neuropathy", in combination with any of the following keywords: "etiology", "pathogenesis", "diagnosis", "prognosis", "treatment", "skin biopsy", "quantitative sensory testing", "nerve conduction study (studies)". Furthermore, the bibliographies of all articles published between 1997 and 2012 regarding SFN were checked. 


\section{References}

1. Langerhans, P. Über die nerven der menschlichen haut [German]. Virchows Arch 44, 325-337 (1868).

2. Weddell, G., Palmer, E. \& Pallie, W. Nerve endings in mammalian skin. Biol. Rev. Camb. Philos. Soc. 30, 63-70 (1955).

3. Lauria, G. Innervation of the human epidermis. A historical review. Ital J Neurol Sci 20, 63-70 (1999).

4. Erlanger, J. \& Gasser, H.S. The compound nature of the action current of nerve as disclosed by the cathode ray oscillograph. Am. J. Physiol. 70, 624-666 (1924).

5. Grant, G. The 1932 and 1944 Nobel Prizes in physiology or medicine: rewards for ground-breaking studies in neurophysiology.J Hist Neurosci 15, 341-57 (2006).

6. McGlone, F. \& Reilly, D. The cutaneous sensory system. Neurosci Biobehav Rev 34, 148-59 (2010).

7. Boulton, A.J., Malik, R.A., Arezzo, J.C. \& Sosenko, J.M. Diabetic somatic neuropathies. Diabetes Care $\mathbf{2 7}$, 1458-86 (2004).

8. Freeman, R. Autonomic peripheral neuropathy. Lancet 365, 1259-70 (2005).

9. Faber, C.G. et al. Gain of function $\mathrm{Na}(\mathrm{V}) 1.7$ mutations in idiopathic small fiber neuropathy. Ann Neurol 71 , 26-39 (2012).

10. Tesfaye, S. et al. Diabetic neuropathies: update on definitions, diagnostic criteria, estimation of severity, and treatments. Diabetes Care 33, 2285-93 (2010).

11. Devigili, G. et al. The diagnostic criteria for small fibre neuropathy: from symptoms to neuropathology. Brain 131, 1912-25 (2008).

12. Holland, N.R. et al. Small-fiber sensory neuropathies: clinical course and neuropathology of idiopathic cases. Ann Neurol 44, 47-59 (1998).

13. Gorson, K.C. et al. Non-length dependent small fibre neuropathy/ganglionopathy. J Neurol Neurosurg Psychiatry 79, 163-9 (2008).

14. Lauria, G. Small fibre neuropathies. Curr Opin Neurol 18, 591-7 (2005).

15. Polydefkis, M. et al. Subclinical sensory neuropathy in late-onset restless legs syndrome. Neurology 55, 1115-21 (2000).

16. Stewart, J.D., Low, P.A. \& Fealey, R.D. Distal small fiber neuropathy: results of tests of sweating and autonomic cardiovascular reflexes. Muscle Nerve 15, 661-5 (1992).

17. Lacomis, D. Small-fiber neuropathy. Muscle Nerve 26, 173-88 (2002).

18. Hoitsma, E. et al. Small fiber neuropathy: a common and important clinical disorder.J Neurol Sci 227, 119-30 (2004).

19. Gorson, K.C. \& Ropper, A.H. Idiopathic distal small fiber neuropathy. Acta Neurol Scand 92, 376-82 (1995).

20. Estacion, M. et al. Intra- and interfamily phenotypic diversity in pain syndromes associated with a gainof-function variant of $\mathrm{NaV}$ 1.7. Mol Pain 7, 92 (2011).

21. Brannagan, T.H., 3rd et al. Small-fiber neuropathy/neuronopathy associated with celiac disease: skin biopsy findings. Arch Neurol 62, 1574-8 (2005).

22. Gemignani, F. et al. Non-length dependent small fiber neuropathy. a prospective case series. J Peripher Nerv Syst 15, 57-62 (2010).

23. Gibbels, E. et al. Severe polyneuropathy in Tangier disease mimicking syringomyelia or leprosy. Clinical, biochemical, electrophysiological, and morphological evaluation, including electron microscopy of nerve, muscle, and skin biopsies. J Neurol 232, 283-94 (1985).

24. Pareyson, D. Diagnosis of hereditary neuropathies in adult patients. J Neurol 250, 148-60 (2003).

25. Burlina, A.P. et al. Early diagnosis of peripheral nervous system involvement in Fabry disease and treatment of neuropathic pain: the report of an expert panel. BMC Neurol 11, 61 (2011).

26. Biegstraaten, $M$. et al. The relation between small nerve fibre function, age, disease severity and pain in Fabry disease. EurJ Pain 15, 822-9 (2011).

27. Plante-Bordeneuve, V. \& Said, G. Familial amyloid polyneuropathy. Lancet Neurol 10, 1086-97 (2011).

28. Bakkers, M. et al. Intraepidermal nerve fiber density and its application in sarcoidosis. Neurology 73, 1142 8 (2009).

29. Malik, R. et al. Small Fiber Neuropathy: Role in the diagnosis of Diabetic Sensorimotor Polyneuropathy. Diabetes Metab Res Rev (2011).

30. Boulais, N. \& Misery, L. The epidermis: a sensory tissue. EurJ Dermatol 18, 119-27 (2008). 
31. Thompson, R.J., Doran, J.F., Jackson, P., Dhillon, A.P. \& Rode, J. PGP 9.5--a new marker for vertebrate neurons and neuroendocrine cells. Brain Res 278, 224-8 (1983).

32. Wang, L., Hilliges, M., Jernberg, T., Wiegleb-Edstrom, D. \& Johansson, O. Protein gene product 9.5-immunoreactive nerve fibres and cells in human skin. Cell Tissue Res 261, 25-33 (1990).

33. Lauria, G. et al. European Federation of Neurological Societies/Peripheral Nerve Society Guideline on the use of skin biopsy in the diagnosis of small fiber neuropathy. Report of a joint task force of the European Federation of Neurological Societies and the Peripheral Nerve Society. Eur J Neurol 17, 903-12, e44-9 (2010).

34. McCarthy, B.G. et al. Cutaneous innervation in sensory neuropathies: evaluation by skin biopsy. Neurology 45, 1848-55 (1995).

35. Lauria, G. et al. Intraepidermal nerve fiber density at the distal leg: a worldwide normative reference study. J Peripher Nerv Syst 15, 202-7 (2010).

36. Chien, H.F. et al. Quantitative pathology of cutaneous nerve terminal degeneration in the human skin. Acta Neuropathol 102, 455-61 (2001).

37. Goransson, L.G., Mellgren, S.I., Lindal, S. \& Omdal, R. The effect of age and gender on epidermal nerve fiber density. Neurology 62, 774-7 (2004).

38. Lauria, G. et al. Morphometry of dermal nerve fibers in human skin. Neurology 77, 242-9 (2011).

39. Vlckova-Moravcova, E., Bednarik, J., Dusek, L., Toyka, K.V. \& Sommer, C. Diagnostic validity of epidermal nerve fiber densities in painful sensory neuropathies. Muscle Nerve 37, 50-60 (2008).

40. Tschachler, E. et al. Sheet preparations expose the dermal nerve plexus of human skin and render the dermal nerve end organ accessible to extensive analysis. J Invest Dermatol 122, 177-82 (2004).

41. Chao, C.C., Sun, H.Y., Chang, Y.C. \& Hsieh, S.T. Painful neuropathy with skin denervation after prolonged use of linezolid. J Neurol Neurosurg Psychiatry 79, 97-9 (2008).

42. Tan, C.H. et al. Painful neuropathy due to skin denervation after metronidazole-induced neurotoxicity. J Neurol Neurosurg Psychiatry 82, 462-5 (2011).

43. Penza, P., Lombardi, R., Camozzi, F., Ciano, C. \& Lauria, G. Painful neuropathy in subclinical hypothyroidism: clinical and neuropathological recovery after hormone replacement therapy. Neurol Sci 30, 149-51 (2009).

44. Ruts, L. et al. Unmyelinated and myelinated skin nerve damage in Guillain-Barre syndrome: correlation with pain and recovery. Pain 153, 399-409 (2012).

45. Sumner, C.J., Sheth, S., Griffin, J.W., Cornblath, D.R. \& Polydefkis, M. The spectrum of neuropathy in diabetes and impaired glucose tolerance. Neurology 60, 108-11 (2003)

46. Smith, A.G., Ramachandran, P., Tripp, S. \& Singleton, J.R. Epidermal nerve innervation in impaired glucose tolerance and diabetes-associated neuropathy. Neurology 57, 1701-4 (2001).

47. Tseng, M.T. et al. Skin denervation and cutaneous vasculitis in systemic lupus erythematosus. Brain 129, 977-85 (2006).

48. Chao, C.C., Hsieh, S.T., Shun, C.T. \& Hsieh, S.C. Skin denervation and cutaneous vasculitis in eosinophiliaassociated neuropathy. Arch Neurol 64, 959-65 (2007).

49. Lombardi, R. et al. IgM deposits on skin nerves in anti-myelin-associated glycoprotein neuropathy. Ann Neurol 57, 180-7 (2005).

50. Simone, D.A., Nolano, M., Johnson, T., Wendelschafer-Crabb, G. \& Kennedy, W.R. Intradermal injection of capsaicin in humans produces degeneration and subsequent reinnervation of epidermal nerve fibers: correlation with sensory function. J Neurosci 18, 8947-59 (1998).

51. Polydefkis, M. et al. The time course of epidermal nerve fibre regeneration: studies in normal controls and in people with diabetes, with and without neuropathy. Brain 127, 1606-15 (2004).

52. Rajan, B., Polydefkis, M., Hauer, P., Griffin, J.W. \& McArthur, J.C. Epidermal reinnervation after intracutaneous axotomy in man. J Comp Neurol 457, 24-36 (2003).

53. Ebenezer, G.J. et al. Impaired neurovascular repair in subjects with diabetes following experimental intracutaneous axotomy. Brain 134, 1853-63 (2011).

54. Ebenezer, G.J. et al. Denervation of skin in neuropathies: the sequence of axonal and Schwann cell changes in skin biopsies. Brain 130, 2703-14 (2007).

55. Hahn, K., Triolo, A., Hauer, P., McArthur, J.C. \& Polydefkis, M. Impaired reinnervation in HIV infection following experimental denervation. Neurology 68, 1251-6 (2007).

56. Kennedy, W.R., Nolano, M., Wendelschafer-Crabb, G., Johnson, T.L. \& Tamura, E. A skin blister method to study epidermal nerves in peripheral nerve disease. Muscle Nerve 22, 360-71 (1999). 
57. Panoutsopoulou, I.G., Wendelschafer-Crabb, G., Hodges, J.S. \& Kennedy, W.R. Skin blister and skin biopsy to quantify epidermal nerves: a comparative study. Neurology 72, 1205-10 (2009).

58. Dyck, P.J. et al. Introduction of automated systems to evaluate touch-pressure, vibration, and thermal cutaneous sensation in man. Ann Neurol 4, 502-10 (1978).

59. Fruhstorfer, H., Lindblom, U. \& Schmidt, W.C. Method for quantitative estimation of thermal thresholds in patients. J Neurol Neurosurg Psychiatry 39, 1071-5 (1976).

6o. Yarnitsky, D. \& Sprecher, E. Thermal testing: normative data and repeatability for various test algorithms. J Neurol Sci 125, 39-45 (1994).

61. Reulen, J.P., Lansbergen, M.D., Verstraete, E. \& Spaans, F. Comparison of thermal threshold tests to assess small nerve fiber function: limits vs. levels. Clin Neurophysiol 114, 556-63 (2003).

62. Hoitsma, E. et al. Abnormal warm and cold sensation thresholds suggestive of small-fibre neuropathy in sarcoidosis. Clin Neurophysiol 114, 2326-33 (2003).

63. Chong, P.S. \& Cros, D.P. Technology literature review: quantitative sensory testing. Muscle Nerve 29, 73447 (2004).

64. Shy, M.E. et al. Quantitative sensory testing: report of the Therapeutics and Technology Assessment Subcommittee of the American Academy of Neurology. Neurology 60, 898-904 (2003).

65. Bakkers, M. Temperature threshold testing and its clinical value in polyneuropathies: a systematic review. J. Peripher. Nerv. Syst. 16, S8 (2011).

66. Oliveira-Soto, L. \& Efron, N. Morphology of corneal nerves using confocal microscopy. Cornea 20, 374-84 (2001).

67. Jalbert, I., Stapleton, F., Papas, E., Sweeney, D.F. \& Coroneo, M. In vivo confocal microscopy of the human cornea. BrJ Ophthalmol 87, 225-36 (2003).

68. Malik, R.A. et al. Corneal confocal microscopy: a non-invasive surrogate of nerve fibre damage and repair in diabetic patients. Diabetologia 46, 683-8 (2003).

69. Hossain, P., Sachdev, A. \& Malik, R.A. Early detection of diabetic peripheral neuropathy with corneal confocal microscopy. Lancet 366, 1340-3 (2005).

70. Quattrini, C. et al. Surrogate markers of small fiber damage in human diabetic neuropathy. Diabetes $\mathbf{5 6}$ 2148-54 (2007).

71. Gemignani, F. et al. Non-length-dependent small fibre neuropathy. Confocal microscopy study of the corneal innervation. J Neurol Neurosurg Psychiatry 81, 731-3 (2010).

72. Lalive, P.H., Truffert, A., Magistris, M.R., Landis, T. \& Dosso, A. Peripheral autoimmune neuropathy assessed using corneal in vivo confocal microscopy. Arch Neurol 66, 403-5 (2009).

73. Tavakoli, M. et al. Corneal confocal microscopy: a novel means to detect nerve fibre damage in idiopathic small fibre neuropathy. Exp Neurol 223, 245-50 (2010).

74. Tavakoli, M. et al. Corneal confocal microscopy: a novel noninvasive means to diagnose neuropathy in patients with Fabry disease. Muscle Nerve 40, 976-84 (2009).

75. Katsarava, Z. et al. A novel method of eliciting pain-related potentials by transcutaneous electrical stimulation. Headache 46, 1511-7 (2006).

76. Casanova-Molla, J., Grau-Junyent, J.M., Morales, M. \& Valls-Sole, J. On the relationship between nociceptive evoked potentials and intraepidermal nerve fiber density in painful sensory polyneuropathies. Pain 152, $410-8$ (2011).

77. Atherton, D.D. et al. Use of the novel Contact Heat Evoked Potential Stimulator (CHEPS) for the assessment of small fibre neuropathy: correlations with skin flare responses and intra-epidermal nerve fibre counts. BMC Neurol 7, 21 (2007).

78. Obermann, M. et al. Correlation of epidermal nerve fiber density with pain-related evoked potentials in HIV neuropathy. Pain 138, 79-86 (2008).

79. Inui, K. \& Kakigi, R. Pain perception in humans: use of intraepidermal electrical stimulation. J Neurol Neurosurg Psychiatry 83, 551-6 (2012).

8o. Bromm, B., Jahnke, M.T. \& Treede, R.D. Responses of human cutaneous afferents to $\mathrm{CO}_{2}$ laser stimuli causing pain. Exp Brain Res 55, 158-66 (1984).

81. Magerl, W., Ali, Z., Ellrich, J., Meyer, R.A. \& Treede, R.D. C- and A delta-fiber components of heat-evoked cerebral potentials in healthy human subjects. Pain 82, 127-37 (1999).

82. Dotson, R.M. Clinical neurophysiology laboratory tests to assess the nociceptive system in humans. J Clin Neurophysiol 14, 32-45 (1997). 
83. Lefaucheur, J.P. \& Creange, A. Neurophysiological testing correlates with clinical examination according to fibre type involvement and severity in sensory neuropathy. J Neurol Neurosurg Psychiatry 75, 417-22 (2004).

84. Truini, A., Romaniello, A., Galeotti, F., lannetti, G.D. \& Cruccu, G. Laser evoked potentials for assessing sensory neuropathy in human patients. Neurosci Lett 361, 25-8 (2004).

85. Agostino, R. et al. Dysfunction of small myelinated afferents in diabetic polyneuropathy, as assessed by laser evoked potentials. Clin Neurophysiol 111, 270-6 (2000).

86. Granovsky, Y., Matre, D., Sokolik, A., Lorenz, J. \& Casey, K.L. Thermoreceptive innervation of human glabrous and hairy skin: a contact heat evoked potential analysis. Pain 115, 238-47 (2005).

87. Arendt-Nielsen, L. \& Chen, A.C. Lasers and other thermal stimulators for activation of skin nociceptors in humans. Neurophysiol Clin 33, 259-68 (2003).

88. Chen, A.C., Niddam, D.M. \& Arendt-Nielsen, L. Contact heat evoked potentials as a valid means to study nociceptive pathways in human subjects. Neurosci Lett 316, 79-82 (2001).

89. Mueller, D. et al. Electrically evoked nociceptive potentials for early detection of diabetic small-fiber neuropathy. EurJ Neurol 17, 834-41 (2010).

90. Serra, J., Campero, M., Bostock, H. \& Ochoa, J. Two types of C nociceptors in human skin and their behavior in areas of capsaicin-induced secondary hyperalgesia.J Neurophysiol 91, 2770-81 (2004).

91. Serra, J. et al. Double and triple spikes in C-nociceptors in neuropathic pain states: an additional peripheral mechanism of hyperalgesia. Pain 152, 343-53 (2011).

92. Serra, J. et al. Microneurographic identification of spontaneous activity in C-nociceptors in neuropathic pain states in humans and rats. Pain 153, 42-55 (2012).

93. Serra, J. et al. C-nociceptors sensitized to cold in a patient with small-fiber neuropathy and cold allodynia. Pain 147, 46-53 (2009).

94. Hilz, M.J. \& Dutsch, M. Quantitative studies of autonomic function. Muscle Nerve 33, 6-20 (2006).

95. Dabby, R., Vaknine, H., Gilad, R., Djaldetti, R. \& Sadeh, M. Evaluation of cutaneous autonomic innervation in idiopathic sensory small-fiber neuropathy. J Peripher Nerv Syst 12, 98-101 (2007).

96. Gibbons, C.H., Illigens, B.M., Wang, N. \& Freeman, R. Quantification of sweat gland innervation: a clinical-pathologic correlation. Neurology 72, 1479-86 (2009).

97. Nolano, M. et al. Quantification of pilomotor nerves: a new tool to evaluate autonomic involvement in diabetes. Neurology 75, 1089-97 (2010).

98. Novak, V. et al. Autonomic impairment in painful neuropathy. Neurology 56, 861-8 (2001).

99. Low, P.A. Evaluation of sudomotor function. Clin Neurophysiol 115, 1506-13 (2004).

100. England, J.D. et al. Evaluation of distal symmetric polyneuropathy: the role of autonomic testing, nerve biopsy, and skin biopsy (an evidence-based review). Muscle Nerve 39, 106-15 (2009).

101. Liguori, R. et al. Microneurographic evaluation of sympathetic activity in small fiber neuropathy. Clin Neurophysiol 122, 1854-9 (2011).

102. Bickel, A. et al. C-fiber axon reflex flare size correlates with epidermal nerve fiber density in human skin biopsies. J Peripher Nerv Syst 14, 294-9 (2009).

103. Bickel, A. et al. Assessment of the neurogenic flare reaction in small-fiber neuropathies. Neurology 59 , 917-9 (2002)

104. Luo, K.R. et al. Quantitation of sudomotor innervation in skin biopsies of patients with diabetic neuropathy. J Neuropathol Exp Neurol 70, 930-8 (2011).

105. Luo, K.R., Chao, C.C., Hsieh, P.C., Lue, J.H. \& Hsieh, S.T. Effect of glycemic control on sudomotor denervation in type 2 diabetes. Diabetes Care 35, 612-6 (2012).

106. Lacomis, D., Giuliani, M.J., Steen, V. \& Powell, H.C. Small fiber neuropathy and vasculitis. Arthritis Rheum 40, $1173-7$ (1997).

107. Polydefkis, M. et al. Reduced intraepidermal nerve fiber density in HIV-associated sensory neuropathy. Neurology 58, 115-9 (2002).

108. Scott, L.J. et al. Quantitative analysis of epidermal innervation in Fabry disease. Neurology 52, 1249-54 (1999).

109. Zambelis, T., Karandreas, N., Tzavellas, E., Kokotis, P. \& Liappas, J. Large and small fiber neuropathy in chronic alcohol-dependent subjects. J Peripher Nerv Syst 10, 375-81 (2005).

110. McManis, P.G., Windebank, A.J. \& Kiziltan, M. Neuropathy associated with hyperlipidemia. Neurology 44 2185-6 (1994).

111. Hoitsma, E. et al. Small fibre neuropathy in sarcoidosis. Lancet 359, 2085-6 (2002). 
112. Gondim, F.A., Brannagan, T.H., 3rd, Sander, H.W., Chin, R.L. \& Latov, N. Peripheral neuropathy in patients with inflammatory bowel disease. Brain 128, 867-79 (2005).

113. Houlden, H., Blake, J. \& Reilly, M.M. Hereditary sensory neuropathies. Curr Opin Neurol 17, 569-77 (2004)

114. Bednarik, J. et al. Etiology of small-fiber neuropathy. J Peripher Nerv Syst 14, 177-83 (2009).

115. Smith, A.G. \& Singleton, J.R. Impaired glucose tolerance and neuropathy. Neurologist 14, 23-9 (2008).

116. Weis, J. et al. Small-fiber neuropathy in patients with ALS. Neurology 76, 2024-9 (2011).

117. Rossi, A., Giovenali, P., Benvenuti, M., Di lorio, W. \& Calabresi, P. Skin biopsy: a new diagnostic tool for autonomic dysfunctions in Parkinson's disease? Lancet Neurol 6, 848-9; author reply 849 (2007).

118. Manganelli, F. et al. Small-fiber involvement in spinobulbar muscular atrophy (Kennedy's disease). Muscle Nerve 36, 816-20 (2007).

119. Oaklander, A.L. et al. Evidence of focal small-fiber axonal degeneration in complex regional pain syndrome-I (reflex sympathetic dystrophy). Pain 120, 235-43 (2006).

120. Lauria, G. et al. Trigeminal small-fiber sensory neuropathy causes burning mouth syndrome. Pain 115 , 332-7 (2005).

121. Penza, P. et al. "Burning tongue" and "burning tip": the diagnostic challenge of the burning mouth syndrome. Clin J Pain 26, 528-32 (2010).

122. Feldman, E.L. Oxidative stress and diabetic neuropathy: a new understanding of an old problem. J Clin Invest 111, 431-3 (2003).

123. Parry, G.J. \& Brown, M.J. Selective fiber vulnerability in acute ischemic neuropathy. Ann Neurol 11, 147-54 (1982).

124. Malik, R.A. et al. Hypoxic neuropathy: relevance to human diabetic neuropathy. Diabetologia 33, 311-8 (1990).

125. Anand, P. et al. The role of endogenous nerve growth factor in human diabetic neuropathy. Nat Med 2 , 703-7 (1996).

126. Krishnan, A.V. \& Kiernan, M.C. Uremic neuropathy: clinical features and new pathophysiological insights. Muscle Nerve 35, 273-90 (2007).

127. Marchand, F., Perretti, M. \& McMahon, S.B. Role of the immune system in chronic pain. Nat Rev Neurosci 6, 521-32 (2005).

128. Pace, M.C. et al. Neurobiology of pain. J Cell Physiol 209, 8-12 (2006).

129. Uceyler, N. et al. Elevated proinflammatory cytokine expression in affected skin in small fiber neuropathy. Neurology 74, 1806-13 (2010).

130. Chamberlain, J.L. et al. Peripherin-IgG association with neurologic and endocrine autoimmunity. J Autoimmun 34, 469-77 (2010).

131. Dabby, R., Weimer, L.H., Hays, A.P., Olarte, M. \& Latov, N. Antisulfatide antibodies in neuropathy: clinical and electrophysiologic correlates. Neurology 54, 1448-52 (2000).

132. Chiang, M.C. et al. Cutaneous innervation in chronic inflammatory demyelinating polyneuropathy. Neurology 59, 1094-8 (2002).

133. Adams, D. Hereditary and acquired amyloid neuropathies. J Neurol 248, 647-57 (2001).

134. Waxman, S.G., Brill, M.H., Geschwind, N., Sabin, T.D. \& Lettvin, J.Y. Probability of conduction deficit as related to fiber length in random-distribution models of peripheral neuropathies. J Neurol Sci 29, 39-53 (1976).

135. Mellion, M., Gilchrist, J.M. \& de la Monte, S. Alcohol-related peripheral neuropathy: nutritional, toxic, or both? Muscle Nerve 43, 309-16 (2011).

136. Koike, H. et al. Alcoholic neuropathy is clinicopathologically distinct from thiamine-deficiency neuropathy. Ann Neurol 54, 19-29 (2003).

137. Cregg, R., Momin, A., Rugiero, F., Wood, J.N. \& Zhao, J. Pain channelopathies. J Physiol 588, 1897-904 (2010).

138. Alessandri-Haber, N. et al. Transient receptor potential vanilloid 4 is essential in chemotherapy-induced neuropathic pain in the rat. J Neurosci 24, 4444-52 (2004).

139. Koivisto, A. et al. Inhibiting TRPA 1 ion channel reduces loss of cutaneous nerve fiber function in diabetic animals: sustained activation of the TRPA 1 channel contributes to the pathogenesis of peripheral diabetic neuropathy. Pharmacol Res 65, 149-58 (2012).

140. Drenth, J.P. \& Waxman, S.G. Mutations in sodium-channel gene SCNgA cause a spectrum of human genetic pain disorders. J Clin Invest 117, 3603-9 (2007). 
141. Davis, M.D. et al. Histopathologic findings in primary erythromelalgia are nonspecific: special studies show a decrease in small nerve fiber density. J Am Acad Dermatol 55, 519-22 (2006).

142. Schiffmann, R. et al. Enzyme replacement therapy improves peripheral nerve and sweat function in Fabry disease. Muscle Nerve 28, 703-10 (2003).

143. Schiffmann, R. et al. Enzyme replacement therapy and intraepidermal innervation density in Fabry disease. Muscle Nerve 34, 53-6 (2006).

144. Dabby, R., Gilad, R., Sadeh, M., Lampl, Y. \& Watemberg, N. Acute steroid responsive small-fiber sensory neuropathy: a new entity? J Peripher Nerv Syst 11, 47-52 (2006).

145. Souayah, N. et al. Effect of intravenous immunoglobulin on cerebellar ataxia and neuropathic pain associated with celiac disease. EurJ Neurol 15, 1300-3 (2008).

146. Attal, N. et al. EFNS guidelines on the pharmacological treatment of neuropathic pain: 2010 revision. Eur J Neurol 17, 1113-e88 (2010).

147. Magri, F. et al. Intraepidermal nerve fiber density reduction as a marker of preclinical asymptomatic small-fiber sensory neuropathy in hypothyroid patients. Eur J Endocrinol 163, 279-84 (2010).

148. Chai, J., Herrmann, D.N., Stanton, M., Barbano, R.L. \& Logigian, E.L. Painful small-fiber neuropathy in Sjogren syndrome. Neurology 65, 925-7 (2005).

149. Oki, Y. et al. Ataxic vs painful form of paraneoplastic neuropathy. Neurology 69, 564-72 (2007).

150. Lund, C. et al. Histopathological and clinical findings in leprosy patients with chronic neuropathic pain: a study from Hyderabad, India. Lepr Rev 78, 369-80 (2007).

151. Bennett, J.L., Mahalingam, R., Wellish, M.C. \& Gilden, D.H. Epstein-Barr virus--associated acute autonomic neuropathy. Ann Neurol 40, 453-5 (1996).

152. Giannoccaro, M.P. et al. Somatic and autonomic small fiber neuropathy induced by bortezomib therapy: an immunofluorescence study. Neurol Sci 32, 361-3 (2011).

153. Heckmann, J.G., Dutsch, M. \& Schwab, S. Linezolid-associated small-fiber neuropathy. J Peripher Nerv Syst 13, 157-8 (2008).

154. Burakgazi, A.Z., Polydefkis, M. \& Hoke, A. Skin biopsy-proven flecainide-induced neuropathy. Muscle Nerve 45, 144-6 (2012).

155. Tan, I.L., Polydefkis, M.J., Ebenezer, G.J., Hauer, P. \& McArthur, J.C. Peripheral nerve toxic effects of nitrofurantoin. Arch Neurol 69, 265-8 (2012).

156. Bergmann, I. et al. Selective degeneration of sudomotor fibers in Ross syndrome and successful treatment of compensatory hyperhidrosis with botulinum toxin. Muscle Nerve 21, 1790-3 (1998).

157. Wouthuis, S.F., van Deursen, C.T., te Lintelo, M.P., Rozeman, C.A. \& Beekman, R. Neuromuscular manifestations in hereditary haemochromatosis. J Neurol 257, 1465-72 (2010).

158. Chao, C.C. et al. Skin denervation and its clinical significance in late-stage chronic kidney disease. Arch Neurol 68, 200-6 (2011).

159. Pan, C.L. et al. Cutaneous innervation in Guillain-Barre syndrome: pathology and clinical correlations. Brain 126, 386-97 (2003).

160. Tembl, J.I. et al. Neurologic complications associated with hepatitis C virus infection. Neurology 53, 861-4 (1999).

161. Younger, D.S. \& Orsher, S. Lyme Neuroborreliosis: Preliminary Results from an Urban Referral Center Employing Strict CDC Criteria for Case Selection. Neurol Res Int 2010, 525206 (2010).

162. Bernstein, A.L. Vitamin B6 in clinical neurology. Ann N Y Acad Sci 585, 250-60 (1990).

163. Nolano, M. et al. Small fibers involvement in Friedreich's ataxia. Ann Neurol 50, 17-25 (2001).

164. Chen, S.F. et al. Neuromuscular abnormality and autonomic dysfunction in patients with cerebrotendinous xanthomatosis. BMC Neurol 11, 63 (2011). 



\title{
Chapter 3
}

\section{Gain-of-function $\mathrm{Na}_{\mathrm{v}} \mathrm{1} \cdot 7$ mutations in idiopathic small fiber neuropathy}

C.G. Faber ${ }^{a *}$, J.G.J. Hoeijmakers ${ }^{a *}$, H.S. Ahn, X. Cheng ${ }^{b, c}$, C. Han ${ }^{b, c}$, J.S. Choib, M. Estacion ${ }^{b, c}$, G. Lauriad ${ }^{d}$, E.K. Vanhoutte ${ }^{a}$, M.M. Gerritse, S.D. Dib-Hajjb,c, J. P.H. Drenthf, S.G. Waxman ${ }^{b, c}$, I.S.J. Merkies ${ }^{a, g}$.

* co-first authorship

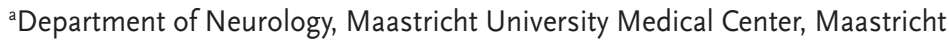
'Department of Neurology, Yale University School of Medicine, New Haven, CT, USA ${ }^{c}$ Center for Neuroscience and Regeneration Research, Veterans Affairs Medical Center,

West Haven, CT, USA

${ }^{d}$ Neuromuscular Diseases Unit, IRCCS Foundation, Carlo Besta, Milan, Italy eDepartment of Clinical Genomics, Maastricht University Medical Center, Maastricht

fDepartment of Gastroenterology and Hepatology, Radboud University Nijmegen Medical Center, Nijmegen 'Department of Neurology, Spaarne Hospital, Hoofddorp
\end{abstract}

Annals of Neurology. 2012;71(1):26-39; Published with permission from John Wiley and Sons 


\section{Abstract}

Objective: Small nerve fiber neuropathy (SFN) often occurs without apparent cause, but no systematic genetic studies have been performed in patients with idiopathic SFN (I-SFN). We sought to identify a genetic basis for I-SFN by screening patients with biopsy-confirmed idiopathic SFN for mutations in the $S C N_{9} A$ gene, encoding voltage gated sodium channel $\mathrm{Na}_{\mathrm{v}}{ }^{1.7}$, which is preferentially expressed in small diameter peripheral axons.

Methods: Patients referred with possible I-SFN, who met the criteria of $\geq 2$ SFNrelated symptoms, normal strength, tendon reflexes, vibration sense, and nerve conduction studies, and reduced intraepidermal nerve fiber density (IENFD) plus abnormal quantitative sensory testing (QST) and no underlying etiology for SFN, were assessed clinically and by screening of $S C N_{9} A$ for mutations and functional analyses.

Results: Twenty-eight patients who met stringent criteria for I-SFN including abnormal IENFD and QST underwent SCNgA gene analyses. Of these 28 patients with biopsy-confirmed I-SFN, 8 were found to carry novel mutations in $\mathrm{SCN}$ gA. Functional analysis revealed multiple gain-of-function changes in the mutant channels; each of the mutations rendered dorsal root ganglion neurons hyperexcitable.

Interpretation: We show for the first time that gain-of-function mutations in sodium channel $\mathrm{Na}_{\mathrm{v}} \mathrm{\gamma} .7$, which render dorsal root ganglion neurons hyperexcitable, are present in a substantial proportion $(28.6 \% ; 8$ of 28$)$ of patients meeting strict criteria for I-SFN. These results point to a broader role of $\mathrm{Na}_{\mathrm{v}} 1.7$ mutations in neurological disease than previously considered from studies on rare genetic syndromes, and suggest an etiological basis for I-SFN, whereby expression of gain-of-function mutant sodium channels in small diameter peripheral axons may cause these fibers to degenerate. 
Small nerve fiber neuropathy (SFN) is a relatively common disorder of thinly myelinated and unmyelinated nerve fibers recently recognized as a distinct clinical syndrome.' The clinical picture is typically dominated by onset in adulthood of neuropathic pain, often with a burning quality, and autonomic symptoms. ${ }^{2 \cdot 6}$ The diagnosis of pure SFN, in which small diameter nerve fibers are affected but large diameter fibers are spared, is usually made on the basis of the clinical picture, preservation of large fiber functions (normal strength, tendon reflexes, and vibration sense), and normal nerve conduction studies (NCS), and is confirmed by demonstration of reduced intraepidermal nerve fiber density (IENFD) or abnormal quantitative sensory testing (QST).7 Despite intensive search for underlying causes such as diabetes mellitus, impaired glucose tolerance, Fabry disease, celiac disease, sarcoidosis, human immunodeficiency virus (HIV), and other systemic illnesses that may be treatable, ${ }^{5,8}$ the proportion of patients with idiopathic SFN (I-SFN), in which no cause can be identified, remains substantial, ranging in different series from $24 \%$ to $93 \% .5,6,9$ Observations of autosomal dominant inheritance suggest a genetic origin for the small fiber involvement that is seen in burning feet syndrome. ${ }^{10}$ However, no specific gene has been linked to, or mutations identified in, patients with adult onset I-SFN.

Voltage-gated sodium channel $\mathrm{Na}_{\mathrm{v}} \mathrm{T} .7$ is preferentially expressed in dorsal root ganglion (DRG) and sympathetic ganglion neurons ${ }^{11,12}$ and their axons, ${ }^{13}$ and opens in response to small depolarizations close to resting potential. ${ }^{14}$ Gainof-function mutations in the $S C N g A$ gene encoding $\mathrm{Na}_{\mathrm{v}} 1.7$ have been found to cause the painful disorders inherited erythromelalgia (IEM) ${ }^{15,16}$ and paroxysmal extreme pain disorder (PEPD), ${ }^{17}$ which are characterized by increased excitability of DRG neurons, and loss of function mutations of $\mathrm{Na}_{\mathrm{v}} 1.7$ have been linked to channelopathy-associated insensitivity to pain. ${ }^{18}$ Sodium channel mutations have not, however, been linked to axonal degeneration. Reasoning that $\mathrm{Na}_{\mathrm{v}}{ }^{1.7}$ is present in small diameter peripheral axons, ${ }^{13}$ in this study we asked whether mutations in the SCNgA gene could be found in a clinically well-defined cohort of patients with biopsy-confirmed I-SFN.

Our results demonstrate, for the first time, the presence of sodium channel mutations in a substantial proportion of patients with I-SFN, show that these missense mutations occur in an ion channel that is preferentially expressed in peripheral axons and share the common feature of rendering DRG neurons hyperexcitable, and point to a broader role of $\mathrm{Na}_{\mathrm{v}} \mathrm{T} .7$ mutations in neurological diseases than previously considered from studies on rare genetic hyperexcitability syndromes. 


\section{Patients and Methods}

\section{Patients}

Inclusion/exclusion criteria: preselection

To accrue a cohort of patients with I-SFN, we initially assessed all patients aged $\geq 18$ years seen at Maastricht University Medical Center neurological clinic with a clinical diagnosis of SFN between 2006 and 2009 and excluded those in whom, after full workup, a cause for SFN was identified. All patients with a clinical diagnosis of I-SFN were asked to participate in this study. Eligibility criteria were normal strength, tendon reflexes, and vibration sense; normal NCS; and presence of at least 2 of the following symptoms: burning feet, allodynia, diminished pain and/or temperature sensation, dry eyes or mouth, orthostatic dizziness, bowel disturbances (constipation/diarrhea/gastroparesis), urinary disturbances, sweat changes (hyper-/hypohidrosis), accommodation problems and/or blurred vision, impotence, diminished ejaculation or lubrication, hot flashes, and palpitations. Exclusion criteria were symptoms or signs of large nerve fiber involvement (muscle weakness, loss of vibration sense, hypo-/ areflexia), abnormal NCS, and history or detection after screening of illnesses known to cause SFN, including diabetes mellitus, impaired glucose tolerance, hyperlipidemia, liver, kidney, or thyroid dysfunction, monoclonal gammopathy, connective tissue disorders, sarcoidosis, Sjogren syndrome, amyloidosis, Fabry disease (alpha-galactosidase, in females combined with GLA gene sequencing), celiac disease, HIV, alcohol abuse, hemochromatosis, antiphospholipid syndrome, B6 intoxication, and neurotoxic drugs (eg, chemotherapy). Patients were not screened for mutations associated with hereditary sensory and autonomic neuropathy, which usually has an early onset and clinical characteristics 19 that are different from those seen in our cohort of patients with I-SFN, or for antibodies to peripherin, which have recently been associated with small fiber neuropathy. ${ }^{20}$

Final patient selection, biopsy, and QST confirmation of SFN

Of 248 patients initially screened following referral with a suspected clinical diagnosis of SFN, 44 patients met inclusion/exclusion criteria and underwent skin biopsy and QST. From this group, 28 met strict criteria for I-SFN (ie, reduced IENFD and abnormal QST compared to normative values). SCN9A gene analysis was carried out in all 28 patients with biopsy-confirmed I-SFN. The current study describes 8 patients, from this group of 28 patients with I-SFN, who were found to carry a mutation in the SCNgA gene (Fig 1). 


\section{Clinical Characterization}

Skin biopsy

Punch biopsy (10cm above lateral malleolus) specimens were fixed ( $2 \%$ paraformaldehyde-lysine-sodium periodate at $4^{\circ} \mathrm{C}$ ), cryoprotected, and stored at $-80^{\circ} \mathrm{C}$ in cryoprotective solution (20\% glycerol) before sectioning $(50 \mu \mathrm{m}) .{ }^{21}$ The numbers of individual nerve fibers crossing the dermal-epidermal junctions were analyzed in each of 3 sections, immunostained with polyclonal rabbit antiprotein gene product-9.5 antibody (Ultraclone; Wellow, Isle-of-Wight, UK), by bright field microscopy using a stereology workstation (Olympus [Tokyo, Japan] BX50, PlanApo oil-objective $x_{40}$ /numerical aperture $\left.(N A)=1.0\right)$. Linear quantification of intraepidermal nerve fiber density was compared with available age- and gender-adjusted normative values. ${ }^{22}$

QST

QST, performed in accordance with previous guidelines, ${ }^{23}$ using a TSA-2001 (Medoc, Ramat-Yishai, Israel) instrument, assessed thresholds at the dorsum of both feet and thenar eminences, using ascending/descending (warm/cool) thermal ramp stimuli delivered through a thermode. ${ }^{24}$ Heat pain modality was also examined. Results were compared with reported normative values. ${ }^{25}$ Measurements were considered abnormal when $Z$ values exceeded 2.5. A sensory modality was classified as abnormal if results of both method of limits and method of levels were abnormal. ${ }^{26}$

SFN symptom inventory questionnaire

The validated SFN Symptom Inventory Questionnaire (SIQ) includes 13 questions (sweating abnormalities, sudden diarrhea, constipation, urination problems (eg, incontinence), dry eyes and/or mouth, orthostatic dizziness, palpitations, hot flashes, skin sensitivity of legs, burning feet, sheet intolerance, and restless legs; each having 4 response options: O 1/4 never, 1 1/4 sometimes, $2 \frac{1}{4}$ often, $3 \frac{1 / 4}{4}$ always) derived from the SIQ22 and a composite autonomic symptoms scale. ${ }^{27}$

Neuropathic pain scale/visual analogue pain scale

The Neuropathic Pain Scale (NPS) was used to assess neuropathic pain, each of 10 qualities being scored from o (no pain) to 10 (most intense pain imaginable). ${ }^{28}$ The Visual Analogue Pain Scale (VAS) ranges from o (no pain) to 100 (most severe pain). ${ }^{29}$ 


\section{SCNgA Mutation Analysis}

Exon screening

Genomic DNA was extracted from $300 \mu \mathrm{L}$ whole blood using the Puregene genomic DNA isolation kit (Gentra-Systems, Minneapolis, MN). All coding exons and flanking intronic sequences, and exons encoding $5^{\prime}$ and $3^{\prime}$ - untranslated sequences within the complementary DNA, were amplified and sequenced as described previously. ${ }^{\circ}$ Genomic sequences were compared with reference $\mathrm{Na}_{\mathrm{v}}$ 1.7 cDNA (NM_002977.3) to identify sequence variations, ${ }^{31}$ using Alamut Mutation-Interpretation Software (Interactive-Biosoftware, Rouen, France). A control panel of DNA from 100 healthy Dutch (Caucasian) individuals (200 chromosomes) was screened for all new mutations.

\section{Functional Analysis}

Previous studies have demonstrated the importance of profiling the effects of $\mathrm{Na}_{\mathrm{v}} 1.7$ mutations on channel function (voltage clamp), and on DRG neuron firing properties (current clamp). ${ }^{15}, 32$ This multimodal analysis of 7 mutations was carried out by 5 electrophysiologists using previously published voltage clamp and current clamp methods in HEK293 cells ${ }^{16}$ and DRG neurons, ${ }^{33}$, 34 transfected with $\mathrm{Na}_{\mathrm{v}} 1.7$ wild-type (WT) or mutant channels as described previously. 34 To minimize inherent culture to culture variation and to overcome the possibility of error introduced by pooling the results of experiments on DRG neurons harvested from multiple animals and cultured over many months, each mutant was compared with contemporaneous controls (WT $\mathrm{Na}_{\mathrm{v}} 1.7$ expressed in cultures of cells prepared, transfected, and recorded under identical conditions by the same electrophysiologist). ${ }^{15}$ Previous studies have demonstrated that some $\mathrm{Na}_{\mathrm{v}} 1.7$ mutations do not produce biophysical changes after expression within heterologous systems such as HEK293 cells, but do produce functional changes after expression in DRG neurons, where voltage clamp is more difficult to achieve due to neurite outgrowth, but the channels are expressed in a native cell background. $.33,34$ Voltage clamp analysis was therefore carried out after transfection into $\mathrm{HEK}_{2} 93$ cells together with $\beta-1$ and $\beta-2$ subunits $^{16}$ or, if biophysical changes were not detected in this cell background, within adult small (<30 $<$ m diameter) DRG neurons. ${ }^{33,34}$ If changes were not found in activation, fast inactivation, slow inactivation, or ramp current, the proportion of cells producing resurgent current, which has been found to be enhanced by some $\mathrm{Na}_{\mathrm{v}} 1.7$ mutations ${ }^{35}$ and the amplitude of resurgent current, was assessed as previously described. ${ }^{35}$ Current clamp analysis was carried out after transfection into DRG neurons. ${ }^{33,34}$ 


\section{Study Design}

The study was approved by medical ethical committees at Yale University and Maastricht University Medical Center. All aspects of the study were explained and written informed consent obtained prior to study. After examination, patients completed the SFN-SIQ, NPS, and VAS in random order. The study was performed between December 2008 and March 2011. Normative values of IENFD were obtained in an earlier study. ${ }^{22}$

\section{Data Analysis}

Clinical characteristics are descriptively presented. Electrophysiological data were analyzed using PulseFit 8.74 (HEKA Electronics, Lambrecht, Germany) or ClampFit (Molecular Devices, Sunnyvale, CA) and Origin 8.1 (Microcal, Northampton, MA), and presented as means \pm standard error. Statistical significance was determined by unpaired Student $t$ tests (voltage clamp except resurgent currents; current clamp except firing frequency and spontaneous activity), Mann-Whitney test (firing frequency), or 2-proportion $z$ test (comparison of proportion of cells producing resurgent currents or spontaneous activity).

\section{Results}

\section{Patient Selection and SCN9A Analysis}

Of 248 Dutch patients referred with a suspected clinical diagnosis of SFN and screened, underlying causes were identified in 185 patients. Nineteen patients were lost to follow-up or refused participation. Forty-four patients met inclusion/ exclusion criteria and underwent skin biopsy and QST. From this group, 28 Dutch Caucasian patients met strict criteria for I-SFN (ie, reduced IENFD compared with age- and gender-adjusted normative values, ${ }^{22}$ plus abnormal QST and no apparent cause) and underwent $S C N_{9} A$ gene analysis (see Fig 1).

Eight (28.6\%) of these 28 patients with biopsy-confirmed I-SFN had mutations in SCNgA (Table 1, Supplementary Table S-1, and Fig 2). In each case, the mutation was missense (c. $554 \mathrm{G}>\mathrm{A}, \mathrm{p} . \mathrm{R} 185 \mathrm{H}$ in 2 unrelated patients; $\mathrm{c} .1867 \mathrm{G}>\mathrm{A}$, p.D623N; c.2215A>C, p.l739V; c.2159T>A, p.l720K; c.4596C>A, p.M1532I; c.2794A>C, p.M932L + c.2971G>T, p.V991L; c.684C>C, p.l228M), and the patient was heterozygous for the mutation. None of these mutations was found in a control panel (DNA from 100 healthy Caucasian Dutch individuals; 200 chromosomes).

Clinical characteristics of the 8 patients with SCNgA mutations (Table 2) were similar to those of the 20 patients without $S C N 9 A$ mutations (Supplementary Table S-2). Here, we describe these 8 patients with I-SFN and SCN9A mutations. 


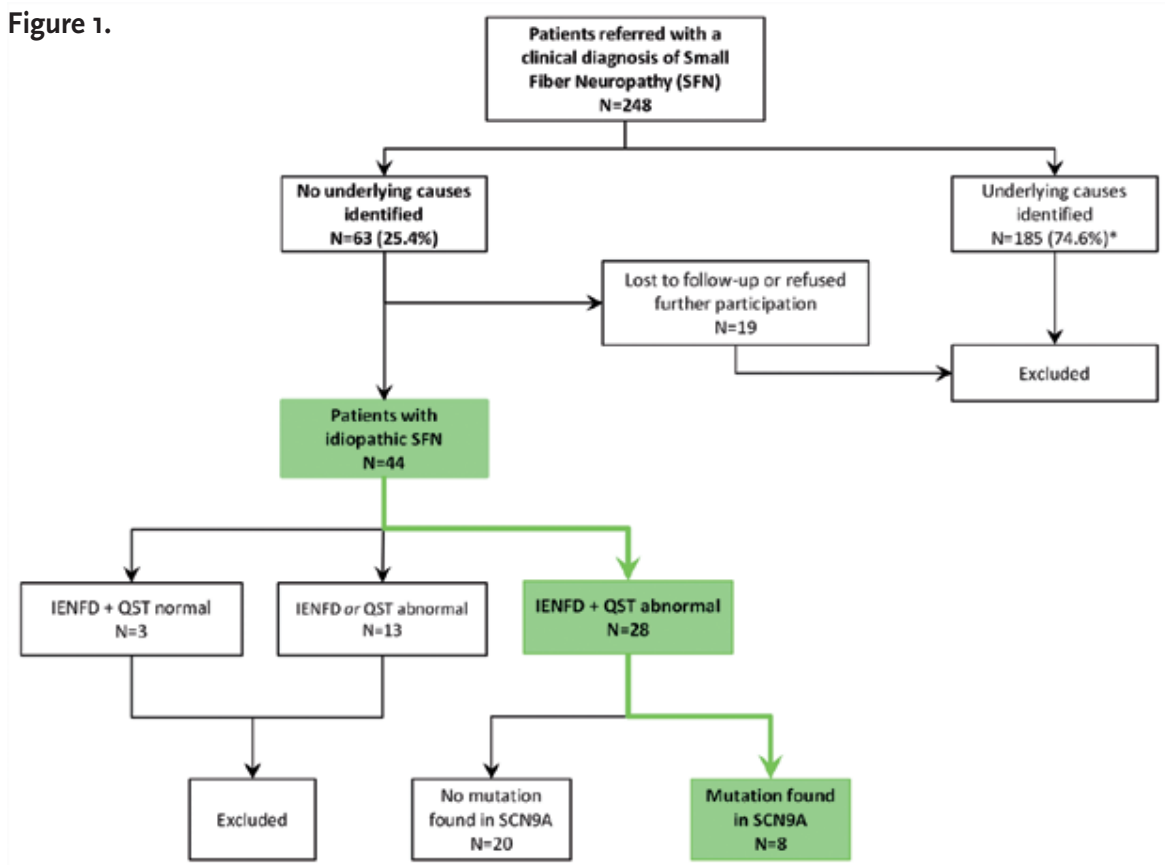

Legend to figure 1. *Causes identified for SFN: sarcoidosis, $n=150$; medication, $n=9$; hemochromatosis, $n=5$; diabetes mellitus, $n=4$; thyroid dysfunction, $n=4$; alcohol abuse, $n=$ 4; gammopathy related, $n=3$; hypercholesterolemia, $n=2$; vitamin $B 6$ intoxication, $n=1$; Lyme disease, $n=1$, Wegener granulomatosis, $n=1$; antiphospholipid syndrome, $n=1$. The Maastricht University Medical Hospital is a referral center for sarcoidosis in the Netherlands. IENFD = intraepidermal nerve fiber density; QST = quantitative sensory testing.

Figure 2.

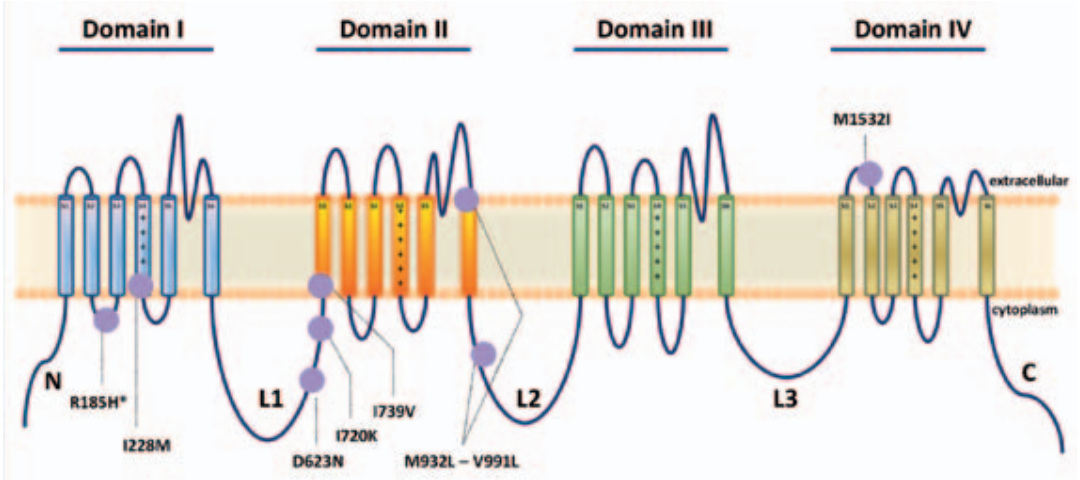

Legend to figure 2. Schematic sodium channel showing the locations of the $\mathrm{Na}_{\mathrm{v}} \mathrm{l} .7$ mutations found in patients with idiopathic small nerve fiber neuropathy. Mutation $\mathrm{R} 185 \mathrm{H}$ was found in 2 patients. 


\section{General Characteristics}

Mean age of these 8 patients with SCNgA mutations was 32.4 (standard deviation [SD], 20.7; median, 23.5; range, 14-68 years; 4 females/4 males). Mean duration of symptoms was 14.5 (SD, 16; range, 1-37) years. Three patients reported similar complaints in family members, but detailed information was not available; family history was unremarkable in 5 patients (see Table 1). Mean age of the 20 patients without $S C N$ gA mutations was 42.7 (SD, 15.4; median, 44 ; range, $7-67$ years; 11 females $/ 9$ males).

\section{Clinical Features}

Pain

All 8 patients complained of pain. Six (patients $1-5,8$ ) had VAS scores $>50$, and 5 (patients 2-6) had scores of $>5$ on at least 7 of the 10 NPS questions, indicating severe pain. Intensity and quality of pain tended to vary from patient to patient. Pain intensity and quality for the 2 patients (patients 1,2) carrying the c.554G>A, p. $\mathrm{R}_{18} \mathrm{H}$ mutation were different from each other. Patient 1 reported less pain compared to patient 2 .

Pain began in the distal extremities (feet $>$ hands) in most patients. However, patients 3 (c.1867G >A, p.D623N) and 5 (c.2159T>A, p.I720K) initially experienced pain throughout the body with muscle ache, before developing distal pain. Pain was aggravated by warmth in 3 of the 8 patients, but not in the other 5 . Cooling relieved pain in 1 patient, but not in the other 7 . Patient 8 (c.684C>C, p.I228M) initially experienced excruciating pain in the teeth/jaw triggered by cold and heat, and pain behind both eyes, not relieved by multiple tooth extractions. He subsequently developed myalgia, aggravated by cold and relieved by warmth, which could persist for 5 to 6 days after light physical activity, and intermittent foot swelling, and was unable to work.

\section{Autonomic dysfunction}

Seven of the 8 patients reported autonomic complaints. In 5 patients, 6 or more of the 9 SFN-SIQ autonomic complaints were present. Orthostatic dizziness, palpitations, dry eyes, and dry mouth were more common (see Table 2). Autonomic complaints were most prominent in patients 4 (c.2215A>G, p.1739V) and 8 (c.684C>G, p.I228M) (see Table 1). Patient 4 experienced dry mouth/eyes, blurred vision, orthostatic dizziness, alternating constipation/ diarrhea, hyperhidrosis, palpitations, hot flashes, and swallowing difficulties, followed by widespread joint/ muscle pain. Patient 8 had a 35-year history of stomach cramps/diarrhea, and dry mouth/eyes and reduced urinary sensation/ hesitation for several years.

Autonomic symptoms were absent in 1 , and much less prominent in the second, of the 2 patients with $\mathrm{R}_{1} 85 \mathrm{H}$ mutation (see Table 2 ). 
Table 1. Clinical description of patients with SFN and $S C N_{9} A$ mutations

\begin{tabular}{|c|c|c|c|c|}
\hline Patient & $\begin{array}{l}\text { Age at } \\
\text { referral/ } \\
\text { Gender }\end{array}$ & $\begin{array}{c}\text { Age at } \\
\text { onset } \\
\text { symptoms }\end{array}$ & $\begin{array}{l}\text { Initial symptom(s) } \\
+ \text { location }\end{array}$ & Later symptoms \\
\hline 1 & $54 /$ male & 24 & $\begin{array}{l}\text { Pain and } \\
\text { paresthesias } \\
\text { feet and hand }\end{array}$ & $\begin{array}{l}52 \text { years: burning feet } \\
\text { 'electrical current' soles and redness feet; } \\
\uparrow \text { with exercise and interfered with walking }\end{array}$ \\
\hline 2 & 24/female & 23 & $\begin{array}{l}\text { Tingling feet, } \\
\text { lower legs and } \\
\text { hands }\end{array}$ & $\begin{array}{l}2 \text { months later: continuous severe pain in feet. } \\
\text { Occasionally: dry mouth and orthostatic dizziness }\end{array}$ \\
\hline 3 & $63 /$ female & 22 & $\begin{array}{l}\text { Painful muscles: } \\
\text { persisting to } \\
\text { present }\end{array}$ & $\begin{array}{l}58 \text { y.o.: severe burning pain, initially soles, } \\
\text { later feet/hands } \\
61 \text { y.o.: patchy skin redness, dry eyes, } \\
\text { dry mouth, orthostatic dizziness } \\
62 \text { y.o.: tenderness and burning of scalp; } \\
\text { burning pain of lips, mouth and trunk }\end{array}$ \\
\hline 4 & 51/female & 14 & $\begin{array}{c}\text { Burning pain, hot } \\
\text { flashes and itching } \\
\text { of face, lower legs } \\
\text { and feet }\end{array}$ & $\begin{array}{c}\text { Complaints with } \uparrow \text { exercise; } \downarrow \text { by cooling } \\
\text { Dry mouth, dry eyes, blurred vision, orthostatic } \\
\text { dizziness, alternating constipation/diarrhea, } \\
\text { hyperhydrosis, palpitations, episodic swallowing } \\
\text { difficulties; redness of hands } \\
49 \text { y.o.: joint and muscle pain }\end{array}$ \\
\hline 5 & $39 /$ male & 37 & $\begin{array}{l}\text { Stabbing pain in } \\
\text { the whole body }\end{array}$ & $\begin{array}{c}2 \text { months later: burning pain feet/lower legs followed by } \\
\text { lower arms. } \\
\text { Numbness feet bilaterally. } \\
\text { Hyperhydrosis in feet, dry mouth, episodic diarrhea, } \\
\text { blurred vision }\end{array}$ \\
\hline 6 & 70/female & 68 & $\begin{array}{l}\text { Stabbing pain } \\
\text { and redness in } \\
\text { the feet, slowly } \\
\text { extending to lower } \\
\text { legs, hands and } \\
\text { lower arms }\end{array}$ & $\begin{array}{l}\text { Symptoms restrict daily activities. } \\
\text { Dry eyes and orthostatic dizziness }\end{array}$ \\
\hline 7 & $22 /$ male & 16 & $\begin{array}{c}\text { Burning pain of } \\
\text { feet and lower legs }\end{array}$ & $\begin{array}{l}\text { Complaints with } \uparrow \text { exercise and interfered with standing. } \\
\text { Orthostatic dizziness, dry mouth, dry eyes, constipation } \\
\text { Sought psychiatric treatment for these symptoms }\end{array}$ \\
\hline 8 & $51 /$ male & 32 & \begin{tabular}{|} 
Excruciating pain \\
in teeth and jaw \\
triggered by cold \\
+ heat, sometimes \\
radiating to \\
temporo- \\
mandibular joint. \\
Also, pain behind \\
the eyes
\end{tabular} & $\begin{array}{l}\text { Multiple tooth extractions did not provide pain relief. } \\
\text { Myalgia triggered by exercise; persisting 5-6 days; } \\
\text { Pain by } \uparrow \text { cold and } \downarrow \text { by warmth (better in summer). } \\
\text { Occasional swollen feet. } \\
\text { For } 35 \text { years: stomach cramps and diarrhea. } \\
\text { For several years: dry mouth, dry eyes, reduced urinary } \\
\text { sensation, and intermittent hesitation. }\end{array}$ \\
\hline
\end{tabular}

Legend to table 1. $R=$ right; $L=$ left; $\uparrow=$ increase; $\downarrow=$ reduced. $Q S T=$ quantitative sensory testing. The obtained QST scores were compared with the reported normative values by Yarnitsky and associates. ${ }^{25} \mathrm{~A}$ sensory modality was classified as abnormal if the results of both method of limit and method of level were abnormal. See also Table S-1 for corresponding findings in relation to normative data. 


\begin{tabular}{|c|c|c|c|c|c|c|}
\hline \multirow{2}{*}{$\begin{array}{l}\text { Aggravated } \\
\text { by warmth/ } \\
\text { Relieved by } \\
\text { cold }\end{array}$} & \multirow{2}{*}{ Family history } & \multirow{2}{*}{ Medication } & \multirow{2}{*}{$\begin{array}{c}\text { IENFD + } \\
\text { (corresponding } \\
\text { normative value) }\end{array}$} & \multicolumn{2}{|c|}{ QST impaired modality } & \multirow{2}{*}{$\begin{array}{l}\text { SCNgA } \\
\text { mutation }\end{array}$} \\
\hline & & & & Thenar & Foot & \\
\hline No/No & $\begin{array}{l}\text { Brother similar } \\
\text { complaints; } \\
\text { Grandfather } \\
\text { painless burns } \\
\text { and walking } \\
\text { difficulty }\end{array}$ & $\begin{array}{c}\text { No effect Pregabalin } \\
\text { and Amytriptyline }\end{array}$ & $\begin{array}{c}1.0 / \mathrm{mm} \\
(\geq 3.2 / \mathrm{mm})\end{array}$ & $\begin{array}{l}\text { Warmth-R } \\
\text { Cold-R } \\
\text { Warmth-L } \\
\text { Cold-L }\end{array}$ & \begin{tabular}{|} 
Warmth-R \\
Cold-R \\
Heat pain-R \\
Warmth-L \\
Cold-L \\
Heat pain-L
\end{tabular} & $\begin{array}{c}\text { C. } 554 \mathrm{G}>\mathrm{A} \\
(\mathrm{R} 185 \mathrm{H})\end{array}$ \\
\hline $\mathrm{No} / \mathrm{No}$ & $\begin{array}{l}\text { Father similar } \\
\text { complaints }\end{array}$ & $\begin{array}{c}\text { No relief } \\
\text { acetaminophen, } \\
\text { anticonvulsants, } \\
\text { antidepressants, } \\
\text { mexiletine, opioids }\end{array}$ & $\begin{array}{c}4.9 / \mathrm{mm} \\
(\geq 6.7 / \mathrm{mm})\end{array}$ & $\begin{array}{l}\text { Warmth-L } \\
\text { Cold-L }\end{array}$ & $\begin{array}{l}\text { Warmth-R } \\
\text { Cold-R } \\
\text { Warmth-L } \\
\text { Cold-L }\end{array}$ & $\begin{array}{c}\text { C. } 554 \mathrm{G}>\mathrm{A} \\
(\mathrm{R} 185 \mathrm{H})\end{array}$ \\
\hline Yes/No & $\begin{array}{c}\text { Sister (78 } \\
\text { years) similar } \\
\text { complaints }\end{array}$ & $\begin{array}{l}\text { Some relief } \\
\text { Pregabalin and } \\
\text { duloxetine }\end{array}$ & $\begin{array}{c}2.8 / \mathrm{mm} \\
(\geq 3.3 / \mathrm{mm})\end{array}$ & - & $\begin{array}{l}\text { Cold-R } \\
\text { Cold-L }\end{array}$ & $\begin{array}{c}\text { c. } 1867 \mathrm{G}>\mathrm{A} \\
(\mathrm{D} 623 \mathrm{~N})\end{array}$ \\
\hline Yes/Yes & $\begin{array}{l}\text { Father, sister, } \\
2 \text { sons, have } \\
\text { similar } \\
\text { complaints }\end{array}$ & $\begin{array}{l}\text { Slight relief } \\
\text { Amytriptyline }\end{array}$ & $\begin{array}{c}3.4 / \mathrm{mm} \\
(\geq 4.1 / \mathrm{mm})\end{array}$ & Warmth-L & $\begin{array}{l}\text { Warmth-L } \\
\text { Cold-L }\end{array}$ & $\begin{array}{c}\text { C. } 2215 \mathrm{~A}>\mathrm{G} \\
(1739 \mathrm{~V})\end{array}$ \\
\hline $\mathrm{No} / \mathrm{No}$ & Unremarkable & No effect Pregabalin & $\begin{array}{c}4.5 / \mathrm{mm} \\
(\geq 4.7 / \mathrm{mm})\end{array}$ & Warmth-L & $\begin{array}{l}\text { Warmth-R } \\
\text { Warmth-L }\end{array}$ & $\begin{array}{c}\text { C.2159T>A } \\
\text { (I720K) }\end{array}$ \\
\hline $\mathrm{No} / \mathrm{No}$ & Unremarkable & No effect Pregabalin & $\begin{array}{c}2.3 / \mathrm{mm} \\
(\geq 2.7 / \mathrm{mm})\end{array}$ & $\begin{array}{l}\text { Warmth-R } \\
\text { Warmth-L }\end{array}$ & $\begin{array}{l}\text { Cold-R } \\
\text { Cold-L }\end{array}$ & $\begin{array}{c}\text { c. } 4596 \mathrm{G}>\mathrm{A} \\
(\mathrm{M} 1532 \mathrm{l})\end{array}$ \\
\hline Yes/No & Unremarkable & $\begin{array}{l}\text { No relief with } \\
\text { Gabapentin }\end{array}$ & $\begin{array}{c}4.0 / \mathrm{mm} \\
(\geq 5.4 / \mathrm{mm})\end{array}$ & - & Warmth-L & $\begin{array}{l}\text { (c.2794A }>C \\
\text { M932L) } \\
\text { and } \\
\text { c. } 2971 \mathrm{G}>\mathrm{T} \\
\text { (V991L) }\end{array}$ \\
\hline $\mathrm{No} / \mathrm{No}$ & $\begin{array}{c}\text { Sister with } \\
\text { rheumatoid } \\
\text { arthritis had } \\
\text { burning hands }\end{array}$ & $\begin{array}{c}\text { Pain bearable with } \\
\text { Acetaminophen; } \\
\text { No relief } \\
\text { antidepressants, } \\
\text { NSAIDs }\end{array}$ & $\begin{array}{c}1.6 / \mathrm{mm} \\
(\geq 3.2 / \mathrm{mm})\end{array}$ & - & $\begin{array}{l}\text { Warmth-R } \\
\text { Cold-R }\end{array}$ & $\begin{array}{c}\text { c. } 684 \mathrm{C}>\mathrm{G} \\
(\mathrm{I} 228 \mathrm{M})\end{array}$ \\
\hline
\end{tabular}


Table S-1. Quantitative sensory testing (QST) in patients with $\mathrm{SCN}_{9} \mathrm{~A}$ novel mutations, findings and corresponding normative data

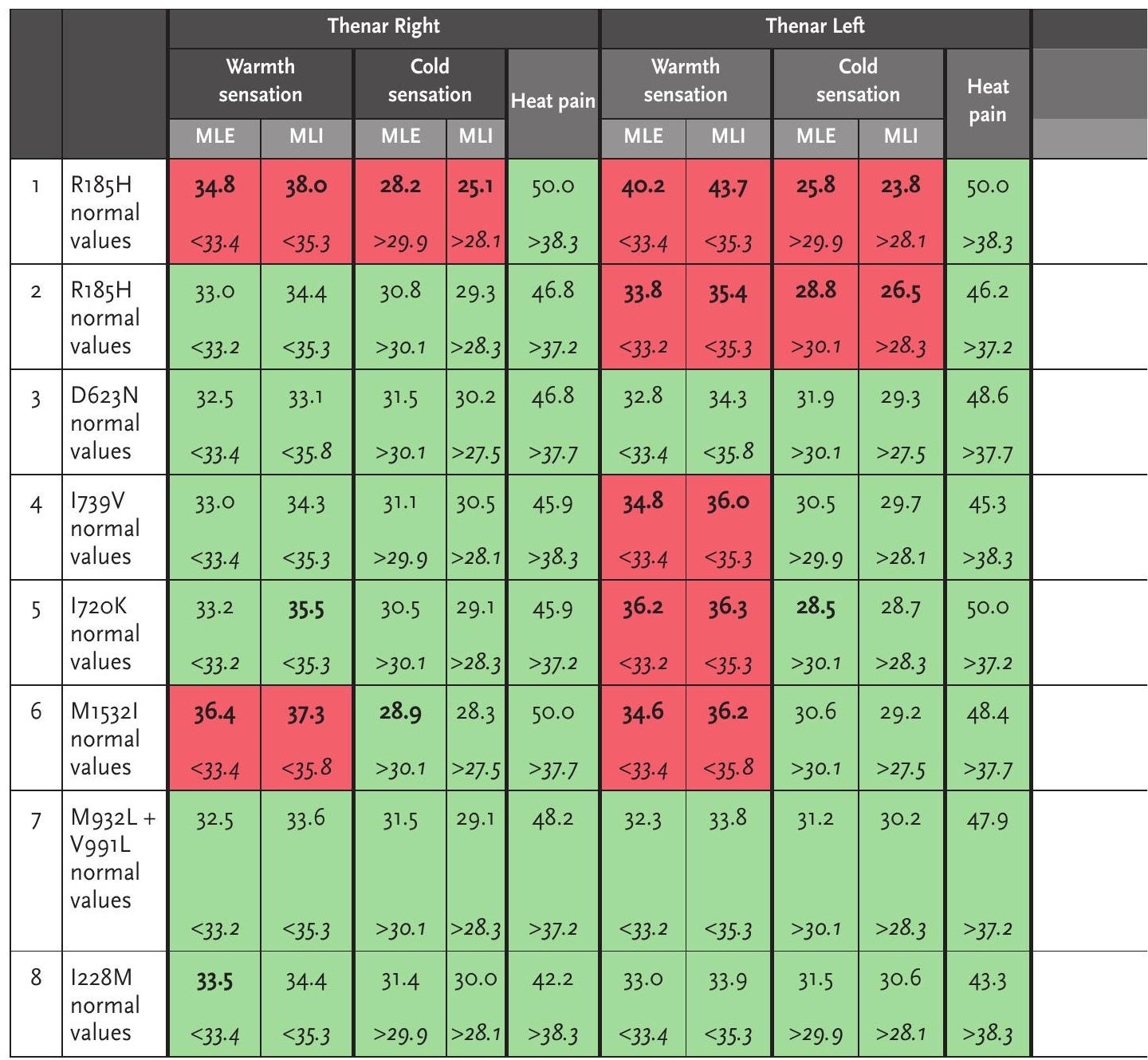

Legend to table S-1. *no QST sensory stimulus was felt. Thresholds were assessed at the dorsum of the foot and thenar eminence bilaterally, using ascending (warm) and descending (cool) thermal energy ramp stimuli delivered through a thermode. Heat pain modality was also examined. Obtained values were compared with reported normative values. A measurement was considered abnormal when its $Z$ value exceeded 2.5 (see Table for cut-off normative values). A sensory modality was classified as abnormal if the results of both method of limit and method of level were abnormal. 


\begin{tabular}{|c|c|c|c|c|c|c|c|c|c|}
\hline \multicolumn{5}{|c|}{ Dorsum foot Right } & \multicolumn{5}{|c|}{ Dorsum foot Left } \\
\hline \multicolumn{2}{|c|}{$\begin{array}{l}\text { Warmth } \\
\text { sensation }\end{array}$} & \multicolumn{2}{|c|}{$\begin{array}{c}\text { Cold } \\
\text { sensation }\end{array}$} & \multirow{2}{*}{ Heat pain } & \multicolumn{2}{|c|}{$\begin{array}{l}\text { Warmth } \\
\text { sensation }\end{array}$} & \multicolumn{2}{|c|}{$\begin{array}{c}\text { Cold } \\
\text { sensation }\end{array}$} & \multirow{2}{*}{$\begin{array}{l}\text { Heat } \\
\text { pain }\end{array}$} \\
\hline MLE & MLI & MLE & MLI & & MLE & MLI & MLE & MLI & \\
\hline$\therefore$ & 50.0 & $\therefore$ & 0.0 & $\therefore$ & $\therefore$ & 50.0 & $\therefore$ & 0.0 & $\therefore$ \\
\hline$<41.3$ & $<45.1$ & $>25.9$ & $>24.5$ & $>40$ & $<41.3$ & $<45.1$ & $>25.9$ & $>24.5$ & $>40$ \\
\hline 44.8 & $47 \cdot 4$ & 25.5 & 23.1 & 49.2 & 46.8 & 47.3 & 23.5 & 17.7 & 50.0 \\
\hline$<39.4$ & $<43.4$ & $>26.6$ & $>25.9$ & $>39.3$ & $<39.4$ & $<43.4$ & $>26.6$ & $>25.9$ & $>39.3$ \\
\hline 37.2 & 37.8 & 12.9 & 16.5 & 48.8 & 38.0 & $39 \cdot 3$ & 21.6 & 20.9 & 48.0 \\
\hline$<41.1$ & $<46.4$ & $>26.1$ & $>26.1$ & $>40.6$ & $<41.1$ & $<46.4$ & $>26.1$ & $>26.1$ & $>40.6$ \\
\hline $43 \cdot 3$ & 44.0 & 26.6 & 24.9 & 48.9 & $44 \cdot 5$ & 46.1 & 21.2 & 18.2 & 49.0 \\
\hline$<41.3$ & $<45.1$ & $>25.9$ & $>25.4$ & $>40$ & $<41.3$ & $<45.1$ & $>25.9$ & $>25.4$ & $>40$ \\
\hline 43.6 & 46.9 & 30.2 & 25.1 & 49.7 & 44.0 & $45 \cdot 5$ & 29.0 & 27.0 & 48.5 \\
\hline$<39.4$ & $<43.4$ & $>26.6$ & $>25.9$ & $>39.3$ & $<39.4$ & $<43.4$ & $>26.6$ & $>25.9$ & $>39.3$ \\
\hline 40.9 & 42.7 & 25.6 & 21.3 & $49 \cdot 9$ & 43.1 & 41.1 & 24.0 & $24 \cdot 7$ & 49.8 \\
\hline$<41.1$ & $<46.4$ & $>26.1$ & $>26.1$ & $>40.6$ & $<41.1$ & $<46.4$ & $>26.1$ & $>26.1$ & $>40.6$ \\
\hline 38.0 & 39.7 & 29.2 & 29.3 & 48.1 & 42.8 & 46.4 & 28.9 & 28.8 & $49 \cdot 3$ \\
\hline$<39.4$ & $<43.4$ & $>26.6$ & $>25.9$ & $>39.3$ & $<39.4$ & $<43.4$ & $>26.6$ & $>25.9$ & $>39.3$ \\
\hline 48.8 & 47.1 & 23.0 & 16.2 & 49.4 & 41.0 & 44.1 & 26.8 & 22.7 & $45 \cdot 4$ \\
\hline$<41.3$ & $<45.1$ & $>25.9$ & $>25.4$ & $>40$ & $<41.3$ & $<45.1$ & $>25.9$ & $>25.4$ & $>40$ \\
\hline
\end{tabular}

Chapter 3. 
Table 2. Small nerve fiber neuropathy symptoms inventory questionnaire (SFN-SIQ) findings in patients with $S C N g A$ novel mutations

\begin{tabular}{|c|c|c|c|c|c|c|c|c|}
\hline $\begin{array}{c}\text { Patient } \\
\text { number }\end{array}$ & sweating & diarrhea & constipation & $\begin{array}{c}\text { micturation } \\
\text { problems }\end{array}$ & dry eyes & $\begin{array}{c}\text { dry } \\
\text { mouth }\end{array}$ & \\
\hline 1 & $\mathrm{R} 185 \mathrm{H}$ & 0 & 0 & 0 & 0 & 0 & 0 & \\
\hline 2 & $\mathrm{R} 185 \mathrm{H}$ & 0 & 0 & 0 & 0 & 0 & 1 & \\
\hline 3 & $\mathrm{D} 623 \mathrm{~N}$ & 0 & 1 & 1 & 0 & 1 & 2 & \\
\hline 4 & $\mathrm{I} 739 \mathrm{~V}$ & 3 & 2 & 1 & 2 & 2 & 3 & \\
\hline 5 & $\mathrm{I} 720 \mathrm{~K}$ & 3 & 1 & 0 & 1 & 1 & 2 & \\
\hline 6 & $\mathrm{M} 1532 \mathrm{l}$ & 0 & 0 & 0 & 0 & 1 & 0 & \\
\hline 7 & $\mathrm{M} 932 \mathrm{~L}+\mathrm{V} 991 \mathrm{~L}$ & 1 & 0 & 2 & 1 & 1 & 1 & \\
\hline 8 & $\mathrm{I} 228 \mathrm{M}$ & 1 & 3 & 1 & 2 & 2 & 3 & \\
\hline
\end{tabular}

Legend to table 2. A green indicates absence (score o) of corresponding SFN related complaint; a red box indicates the presence of SFN related symptom, with variable intensity (score 1: sometimes present; score 2: often, and a score 3: always present)

Table S-2. Small nerve fiber neuropathy symptoms inventory questionnaire (SFN-SIQ) findings in patients without $\mathrm{SCN}$ gA novel mutations

\begin{tabular}{|c|c|c|c|c|c|c|}
\hline Patient & sweating & diarrhea & constipation & $\begin{array}{l}\text { micturation } \\
\text { problems }\end{array}$ & $\begin{array}{l}\text { dry } \\
\text { eyes }\end{array}$ & $\begin{array}{c}\text { dry } \\
\text { mouth }\end{array}$ \\
\hline 1 & 0 & 0 & 0 & 0 & 2 & 1 \\
\hline 2 & 2 & 1 & 1 & 2 & 2 & 2 \\
\hline 3 & 0 & 0 & 0 & 0 & 0 & 1 \\
\hline 4 & 2 & 1 & 3 & 3 & 2 & 2 \\
\hline 5 & 0 & 0 & 2 & 3 & 0 & 1 \\
\hline 6 & 0 & 1 & 0 & 0 & 0 & 1 \\
\hline 7 & 0 & 0 & 0 & 0 & 0 & 0 \\
\hline 8 & 1 & 0 & 3 & 2 & 2 & 2 \\
\hline 9 & 0 & 0 & 0 & 1 & 1 & 0 \\
\hline 10 & 2 & 0 & 3 & 2 & 2 & 2 \\
\hline 11 & 3 & 0 & 2 & 3 & 0 & 3 \\
\hline 12 & 0 & 1 & 0 & 0 & 1 & 1 \\
\hline 13 & 0 & 0 & 0 & 0 & 0 & 1 \\
\hline 14 & 0 & 0 & 0 & 0 & 0 & 0 \\
\hline 15 & 1 & 0 & 1 & 1 & 0 & 1 \\
\hline 16 & 1 & 0 & 2 & 2 & 0 & 2 \\
\hline 17 & 3 & 1 & 0 & 1 & 3 & 3 \\
\hline 18 & 1 & 3 & 0 & 0 & 2 & 0 \\
\hline 19 & 1 & 0 & 0 & 1 & 0 & 0 \\
\hline 20 & 1 & 0 & 0 & 1 & 0 & 0 \\
\hline
\end{tabular}

Legend to table S-2. A green indicates absence (score o) of corresponding SFN related complaint; a red box indicates the presence of SFN related symptom, with variable intensity (score 1 : sometimes present; score 2: often, and a score 3: always present). There were 9 females and 1 males, age 53.6 (SD 14.4), range $19-76$ years, duration of symptoms: 9.1 (SD 7.4), range $1-30$ years. 
orthostatic

palpitations hot flashes skin hyperesthesia burning feet

sheet

restless

dizziness

intolerance

legs

\begin{tabular}{|l|l|l|l|l|l|l|}
\hline 0 & 0 & 0 & 1 & 2 & 3 & 3 \\
\hline 1 & 0 & 0 & 3 & 3 & 2 & 2 \\
\hline 2 & 2 & 0 & 2 & 2 & 2 & 2 \\
\hline 1 & 1 & 3 & 2 & 2 & 2 & 2 \\
\hline 0 & 0 & 1 & 2 & 1 & 1 & 1 \\
\hline 1 & 1 & 0 & 3 & 3 & 1 & 3 \\
\hline 0 & 1 & 1 & 1 & 2 & 0 & 0 \\
\hline
\end{tabular}

Chapter

3.

\begin{tabular}{|c|c|c|c|c|c|c|}
\hline $\begin{array}{c}\text { orthostatic } \\
\text { dizziness }\end{array}$ & palpitations & $\begin{array}{c}\text { hot } \\
\text { flashes }\end{array}$ & $\begin{array}{c}\text { skin } \\
\text { hyperesthesia }\end{array}$ & $\begin{array}{c}\text { burning } \\
\text { feet }\end{array}$ & $\begin{array}{c}\text { sheet } \\
\text { intolerance }\end{array}$ & restless legs \\
\hline 2 & 0 & 0 & 1 & 3 & 2 & 2 \\
\hline 2 & 2 & 3 & 3 & 3 & 3 & 3 \\
\hline 0 & 0 & 0 & 1 & 2 & 1 & 2 \\
\hline 2 & 2 & 2 & 3 & 3 & 3 & 3 \\
\hline 0 & 1 & 0 & 3 & 0 & 1 & 2 \\
\hline 1 & 3 & 0 & 3 & 1 & 2 & 2 \\
\hline 0 & 0 & 1 & 3 & 1 & 1 & 3 \\
\hline 0 & 1 & 1 & 3 & 2 & 2 & 1 \\
\hline 1 & 1 & 0 & 2 & 3 & 0 & 2 \\
\hline 1 & 1 & 1 & 3 & 3 & 1 & 2 \\
\hline 0 & 0 & 1 & 3 & 2 & 2 & 1 \\
\hline 0 & 0 & 2 & 3 & 3 & 3 & 3 \\
\hline 1 & 1 & 0 & 0 & 2 & 1 & 0 \\
\hline 0 & 0 & 1 & 1 & 3 & 0 & 3 \\
\hline 0 & 0 & 0 & 2 & 3 & 3 & 2 \\
\hline 0 & 0 & 1 & 3 & 3 & 3 & 2 \\
\hline 1 & 0 & 0 & 3 & 3 & 1 & 3 \\
\hline 1 & 0 & 1 & 2 & 2 & 1 & 0 \\
\hline 1 & 1 & 1 & 0 & 1 & 0 & 1 \\
\hline 1 & 1 & 1 & 0 & 2 & 2 & 1 \\
\hline
\end{tabular}




\section{IENFD and QST findings}

There was a decrease in IENFD below the 5th percentile for age- and sexmatched controls $^{22}$ in all 8 patients with SCNgA mutations (see Table 1). Supplementary Figure 1 shows the IENFD findings in patient 8 juxtaposed to an age- and gender-matched control subject.

On QST, 5 patients displayed abnormal warm and cold sensation, 1 patient displayed abnormal warm sensation, and 2 displayed abnormal cold sensation. One patient displayed reduced heat pain (see Table 1; Supplementary Table S-1). More abnormalities were seen in the foot $(21$ of 48 sensory qualities tested, $43.8 \%$ ) compared with the hand (10 of $48,20.8 \%$ ).

\section{Functional Characterization of $\mathrm{Na}_{\mathrm{v}} \mathrm{1.7}$ Mutations}

Voltage clamp analysis of the mutant channels from patients with I-SFN showed that they were all gain-of-function, and that they impaired slow inactivation (p.I720K, p.M1532I, p.I228M, p.I739V), depolarized slow and fast inactivation (p.D623N), or enhanced resurgent currents (p.M932L/V991L, p.R185H). None of these mutations exhibited the hyperpolarized activation or enhanced ramp currents characteristic of IEM ${ }^{15}$ or the incomplete fast inactivation characteristic of PEPD ${ }^{17}$ mutations of $\mathrm{Na}_{\mathrm{v}}{ }^{1.7}$. Current clamp analysis demonstrated that all 7 mutations rendered DRG neurons hyperexcitable. Here we present the functional profiling of 3 representative mutant channels from patients with I-SFN. Functional profiling of the other 4 mutations yielded similar results (unpublished results).

\section{I720K: Impaired Slow Inactivation and DRG Neuron Hyperexcitability}

Voltage clamp analysis of $1720 \mathrm{~K}$ mutant channels following expression in HEK293 cells demonstrated impaired slow inactivation (Fig $3 \mathrm{C}$ ). Current densities (WT: $375 \pm 68 \mathrm{pA} / \mathrm{pF}, \mathrm{n}=18$; I720K: $228 \pm 35 \mathrm{pA} / \mathrm{pF}, \mathrm{n}=22$ ), activation $\mathrm{V}_{1 / 2}\left(\mathrm{~V}_{1 / 2}\right.$ represents voltage midpoint) (WT: $-26.6 \pm 1.6 \mathrm{mV}, \mathrm{n}=12$; I720K: -25.8 $\pm 0.9 \mathrm{mV}, \mathrm{n}=13)$, fast inactivation $\mathrm{V}_{1 / 2}(\mathrm{WT}:-80.4 \pm 1.5 \mathrm{mV}, \mathrm{n}=13$; $1720 \mathrm{~K}:-79.1$ $\pm 1.1 \mathrm{mV}, \mathrm{n}=13$ ), and ramp currents (WT: $0.8 \pm 0.2 \%, \mathrm{n}=9$; I $1720 \mathrm{~K}: 0.7 \pm 0.1 \%$, $n=10)$ for HEK293 cells transfected with WT or I720K were not significantly different. Slow inactivation was impaired for $1720 \mathrm{~K}$ mutant channels, with a depolarized $\mathrm{V}_{1 / 2}$ (WT: $-73.2 \pm 2.4 \mathrm{mV}, \mathrm{n}=6$; I720K: $-64.4 \pm 1.5 \mathrm{mV}, \mathrm{n}=7 ; \mathrm{p}<$ 0.05 ; see Fig $3 \mathrm{C}$ ). Impaired slow inactivation increases the number of channels available for activation at potentials positive to $-100 \mathrm{mV}$, including potentials close to resting potential of DRG neurons.

The l720K mutation had clear functional effects on DRG neurons, which were rendered hyperexcitable by the mutant channels (see Fig 3). I720K produced a depolarizing shift in resting membrane potential (WT: $-55.8 \pm 1.7 \mathrm{mV}, n=26$; I720K: $-48.7 \pm 1.9 \mathrm{mV}, \mathrm{n}=29 ; \mathrm{p}<0.05)$. I720K increased excitability of DRG neurons, 
Figure 3.

A1

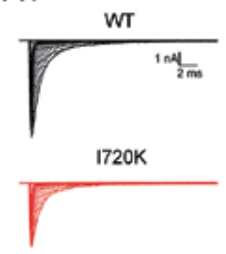

A4

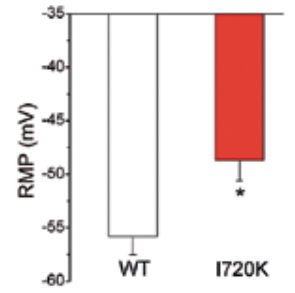

A2

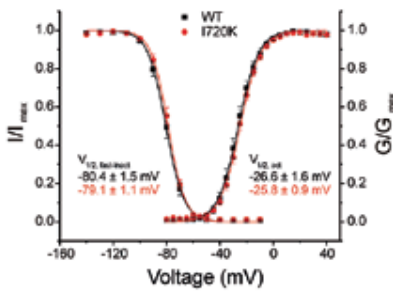

A5

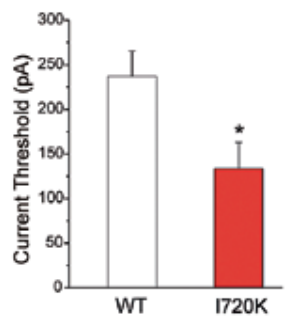

A3

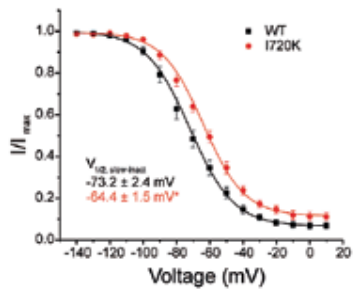

A6

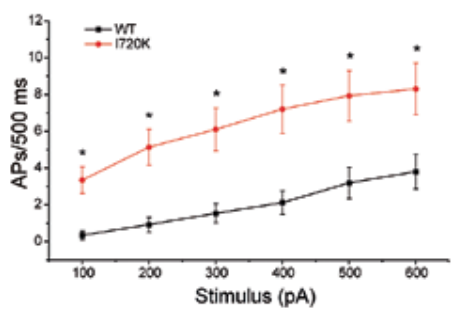

A7

\section{I720K}

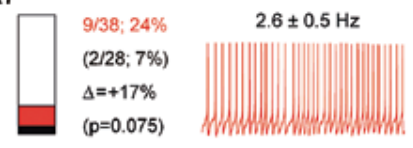

Legend to figure 3. Electrophysiological analysis of $1720 \mathrm{~K}$ mutation. (A) Representative current traces recorded from HEK 293 cells expressing wild type (WT) (top) or I720K (bottom), evoked by voltage steps (100 milliseconds) from -80 to $40 \mathrm{mV}$ in $5 \mathrm{mV}$ increments, from a holding potential of $-120 \mathrm{mV}$. (B) Activation and steady state fast inactivation for WT (black squares) and I720K (red circles). Fast inactivation was examined using a series of 500-millisecond prepulses from -140 to $-10 \mathrm{mV}$ followed by test pulses to -10mV. Left inset: midpoint values for fast inactivation $\left(\mathrm{V}_{1 / 2, \text { fast-inact }}\right)$ of WT (black) and I720K (red). Right inset: midpoint values for activation ( $\mathrm{V}_{1 / 2 \text {, act }}$ of WT (black) and I720K (red). (C) Steady state slow inactivation of WT (black squares) and I720K (red circles). Slow inactivation was assessed using a 20-millisecond pulse to -10mV after a 30-second prepulse to potentials from -140 to $10 \mathrm{mV}$ followed by a 100-millisecond pulse to $-120 \mathrm{mV}$ to remove fast inactivation. Inset: midpoint values of slow inactivation ( $\mathrm{V}_{1 / 2, \text { slow-inact) }}$ (WT: black; I720K: red); *p < 0.05 . (D) Resting membrane potential (RMP) of dorsal root ganglion (DRG) neurons expressing WT $(-55.8 \pm 1.7, n=26)$ or I720K $(-48.7 \pm 1.9, n=29)$; *p $<0.05$. (E) Current threshold of DRC neurons expressing WT $(237 \pm 28, n=26)$ or I720K (134 $\pm 30, n=29)$ to 500 -millisecond stimuli; $\mathrm{p}<0.05$. (F) Comparison of mean firing frequency in DRG neurons expressing WT and I720K across a range of current injections from 100 to 60opA; *p < 0.05. (G) Bar graph showing the proportion of spontaneous firing cells for DRG neurons expressing I720K (red) and WT channels (black); numbers to the right of the bar graph show mean values for WT (lower value in parentheses) and I720K (upper value). The recording on the right shows spontaneous firing (10 seconds) of representative DRG neuron expressing I720K; the numbers above the trace show average \pm standard deviation frequency of spontaneous action potentials. $V_{1 / 2}$ represents voltage midpoint, I/I represents normalized current, and $\mathrm{G} / \mathrm{G}$ represents normalized conductance for fast-activation, slow inactivation, and activation. $\mathrm{APs}=$ action potentials. 
with a $43 \%$ reduction in current threshold to 500-millisecond stimuli (WT: $237 \pm$ 28pA, $n=26$; I720K: $134 \pm 30 p A, n=29 ; p<0.05)$. I720K significantly increased the number of action potentials evoked by 500 -millisecond depolarizing stimuli at all intensities tested, from 100 to $600 \mathrm{pA}$. I720K produced a trend toward an increase in the proportion of spontaneously firing cells (9 of 38 [24\%] vs 2 of 28 [7\%] for cells transfected with WT channels) that did not reach statistical significance $(p=0.075)$; mean frequency of spontaneous activity in cells transfected with $1720 \mathrm{~K}$ was $2.6 \pm 0.5 \mathrm{~Hz}(n=9)$, with 4 of 9 spontaneously firing cells showing continuous firing at a frequency of $>1 \mathrm{~Hz}$ throughout the 30-second recording period.

D623N: Impaired Fast and Slow Inactivation and DRG Neuron Hyperexcitability D623N mutant channels did not display gating abnormalities following expression in HEK293 cells but, when assessed by voltage clamp after expression in DRG neurons, demonstrated impaired fast inactivation and slow inactivation (Fig 4A-C). Current densities (WT: $407 \pm 90 p A, n=12$; D623N: $474 \pm 121 \mathrm{pA}$, $\mathrm{n}=10$ ), activation $\mathrm{V}_{1 / 2}(\mathrm{WT}:-26.9 \pm 2.0 \mathrm{mV}, \mathrm{n}=12 ; \mathrm{D} 623 \mathrm{~N}:-26.4 \pm 2.3 \mathrm{mV}, \mathrm{n}=$ 10), and ramp currents (WT: $2.6 \pm 0.4 \%, n=15 ; D 623 \mathrm{~N}: 2.1 \pm 0.3 \%, n=17$ ) were not significantly different. The $\mathrm{V}_{1 / 2}$ of fast inactivation (WT: $-76.7 \pm 1.4 \mathrm{mV}$, $\mathrm{n}=13$; D623N: $-72.2 \pm 1.2 \mathrm{mV}, \mathrm{n}=13 ; \mathrm{p}<0.05$ ) and slow inactivation (WT: -69.6 $\pm 1.4 \mathrm{mV}, \mathrm{n}=12 ; \mathrm{D} 623 \mathrm{~N}:-64.3 \pm 2.0 \mathrm{mV}, \mathrm{n}=11 ; \mathrm{p}<0.05)$ were depolarized for D623N mutant channels (see Fig 4B, C). Impaired fast inactivation and slow inactivation increase the number of channels available for activation.

Current clamp recording showed that D623N mutant channels rendered DRG neurons hyperexcitable and produced aberrant spontaneous firing in $25 \%$ of neurons (see Fig 4). D623N produced a depolarizing shift in resting membrane potential (WT: $-55.0 \pm 1.5 \mathrm{mV}, \mathrm{n}=29 ; \mathrm{D} 623 \mathrm{~N}:-45.5 \pm 1.5 \mathrm{mV}, \mathrm{n}=27 ; \mathrm{p}<0.01$ ) and a $51 \%$ reduction in current threshold to 200 -millisecond stimuli (WT: $256 \pm 28 \mathrm{pA}$, $\mathrm{n}=29$; $\mathrm{D} 623 \mathrm{~N}: 125 \pm 19 \mathrm{pA}, \mathrm{n}=27 ; \mathrm{p}<0.01)$. D623N significantly increased the number of action potentials evoked by 500 -millisecond depolarizing stimuli ranging from 100 to $275 \mathrm{pA}$. D623 N produced an increase in the proportion of spontaneously firing cells ( 9 of 36 [25\%] for DRG neurons transfected with this mutant channel; 1 of 30 [3\%] for cells transfected with WT channels, $p<0.05$ ). Mean frequency of spontaneous activity in cells transfected with $D 623 \mathrm{~N}$ was 2.4 $\pm 0.6 \mathrm{~Hz}(n=9) ; 4$ of 9 spontaneously firing cells showed continuous firing at a frequency of $>1 \mathrm{~Hz}$ throughout the 30-second recording period. 
B1

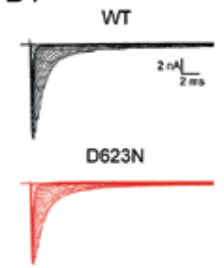

B4

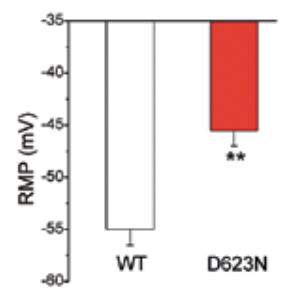

B2

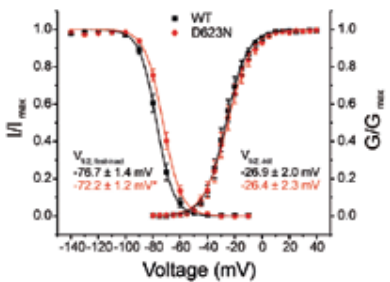

B5

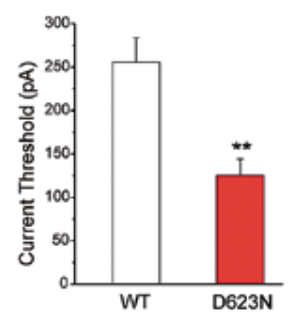

B3

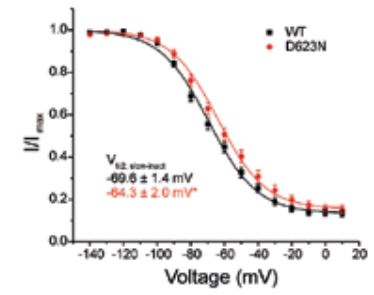

B6

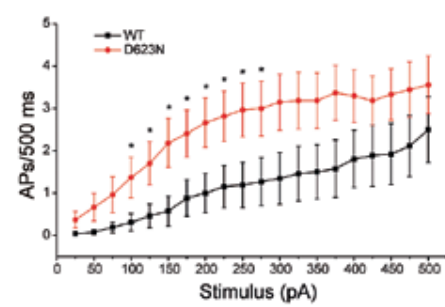

B7

$2.4 \pm 0.6 \mathrm{~Hz}$

D623N

Chapter

3.

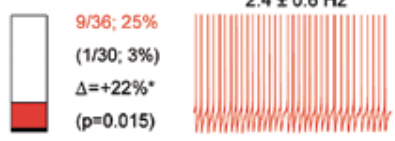

Legend to figure 4. Electrophysiological analysis of D623N mutation. (A) Representative current traces recorded from dorsal root ganglion (DRG) neurons expressing wild type (WT) (top) or $\mathrm{D} 623 \mathrm{~N}$ (bottom), evoked by voltage steps (100 milliseconds) from -80 to $40 \mathrm{mV}$ in $5 \mathrm{mV}$ increments, from a holding potential of $-100 \mathrm{mV}$. (B) Activation and steady state fast inactivation for WT (black squares) and D623N (red circles). Fast inactivation was examined using a series of 500-millisecond prepulses from -140 to $-10 \mathrm{mV}$ followed by test pulses to -10mV. Left inset: midpoint values for fast inactivation ( $\left.\mathrm{V}_{1 / 2 \text {, fast-inact }}\right)$ of $\mathrm{WT}$ (black) and $\mathrm{D} 623 \mathrm{~N}$ (red). Right inset: midpoint values for activation $\left(\mathrm{V}_{1 / 2}\right.$, act $)$ of WT (black) and D623N (red). (C) Steady state slow inactivation of WT (black squares) and D623N (red circles). Slow inactivation was assessed using a 20-millisecond pulse to -10mV after a 30-second prepulse to potentials from -140 to $10 \mathrm{mV}$ followed by a 100-millisecond pulse to -120mV to remove fast inactivation. Inset: midpoint values

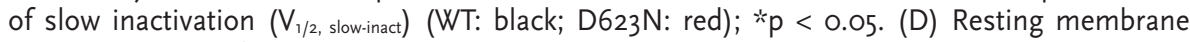
potential (RMP) of DRG neurons expressing WT $(-55.0 \pm 1.5, n=29)$ or D623N $(-45.5 \pm 1.5, n$ $=27)$, **p $<0.01$. (E) Current threshold of DRG neurons expressing WT $(256 \pm 28, n=29)$ or D623N (125 $\pm 19, n=27)$ to 200-millisecond stimuli; **p $<0.01$. (F) Comparison of mean firing frequency in DRG neurons expressing WT and D623 $\mathrm{N}$ across a range of current injections from 25 to $500 \mathrm{pA}$; *p < 0.05. (G) Bar graph showing the proportion of spontaneous firing cells for DRC neurons expressing D623N (red) and WT channels (black); numbers to the right of the bar graph show mean values for WT (lower value in parentheses) and D623N (upper value); * $p<0.05$. The recording on the right shows spontaneous firing (10 seconds) of representative DRG neuron expressing $\mathrm{D} 623 \mathrm{~N}$; the numbers above the trace show the average \pm standard deviation frequency of spontaneous action potentials. $V_{1 / 2}$ represents voltage midpoint, $1 / 1$ represents normalized current, and $\mathrm{G} / \mathrm{G}$ represents normalized conductance for fast-activation, slow-inactivation, and activation. $\mathrm{APs}=$ action potentials. 
M932L/V991L: Increased Resurgent Currents and DRG Neuron Hyperexcitability M932L/V991L mutant channels, assessed by voltage clamp after expression in DRG neurons (Fig 5A-D), enhanced the generation of resurgent currents. Voltage clamp analysis of M932L/V991L mutant channels, both in HEK293 cells and DRG neurons, did not reveal a significant effect of the mutation on activation, fast inactivation, slow inactivation, ramp currents, or deactivation. Current densities (WT: $440 \pm 49 \mathrm{pA} / \mathrm{pF}, \mathrm{n}=32 ; \mathrm{M} 932 \mathrm{~L} / \mathrm{V} 991 \mathrm{~L}: 541 \pm 86 \mathrm{pA} / \mathrm{pF}$, $\mathrm{n}=23)$, activation $\mathrm{V}_{1 / 2}(\mathrm{WT}:-20.2 \pm 0.6 \mathrm{mV}, \mathrm{n}=16 ; \mathrm{Mg32L} / \mathrm{V} 991 \mathrm{~L}:-20.4 \pm 1.2 \mathrm{mV}$, $\mathrm{n}=11)$, fast inactivation $\mathrm{V}_{1 / 2}$ (WT: $-68.560 .6 \mathrm{mV}, \mathrm{n}=26$; M932L/V991 L: -68.5 $\pm 0.6 \mathrm{mV}, \mathrm{n}=17)$, slow inactivation $\mathrm{V}_{1 / 2}(\mathrm{WT}:-66.1 \pm 1.0 \mathrm{mV}, \mathrm{n}=24 ; \mathrm{M} 932 \mathrm{~L} /$ V991L: $-63.8 \pm 1.7 m V, n=16)$, ramp currents (WT: $1.61 \pm 0.16 \%, n=19 ; \mathrm{M} 932 \mathrm{~L}$ / V991L: $1.85 \pm 0.27 \%, n=14$ ), and deactivation (no significant differences in deactivation measured between -100 and $-50 \mathrm{mV}$ at $5 \mathrm{mV}$ intervals) for DRC neurons transfected with $\mathrm{WT}$ and $\mathrm{M} 932 \mathrm{~L} / \mathrm{V} 991 \mathrm{~L}$ were not significantly different. However, a higher percentage of DRG neurons expressing M932L/V991L (5 of 10 cells, 50\%; $p<0.05$ ) compared to cells expressing WT channels (1 of 11 cells, $9 \%$; see Fig $5 \mathrm{D}$ ) produced resurgent currents, a change that would be expected to produce repetitive firing.

Current clamp recording showed that M932L/V991 L mutant channels made DRG neurons hyperexcitable. Mean resting potential was significantly depolarized (WT: $-56.9 \pm 1.9 \mathrm{mV}, \mathrm{n}=20$; M932L/V991L: $-49.8 \pm 1.6 \mathrm{mV}, \mathrm{n}=23$, $p<0.01$, and threshold was significantly decreased (WT: $250 \pm 23 \mathrm{pA}, \mathrm{n}=20$; M932L/V991 L: $145 \pm 22 \mathrm{pA}, \mathrm{n}=23, \mathrm{p}<0.01$ in DRG neurons expressing M932L/ $\mathrm{V}_{991 \mathrm{~L}}$ (see Fig 5). The number of action potentials evoked by 500 -millisecond depolarizing stimuli was increased at all stimulus strengths between 50 and 300pA for cells expressing M932L/V991 L channels, compared to cells expressing WT. M932L/V991L produced a trend toward an increase in the proportion of spontaneously firing cells ( 4 of 27 [15\%] vs o of 20 [0\%] for cells transfected with WT channels) that did not reach statistical significance $(p=0.072)$; mean frequency of spontaneous activity in cells transfected with M932L/V991 L was $2.0 \pm 0.3 \mathrm{~Hz}$, with all 4 spontaneously firing cells showing continuous firing at a mean frequency of $>1 \mathrm{~Hz}$ throughout the 30 -second recording period. 
Figure 5

A1
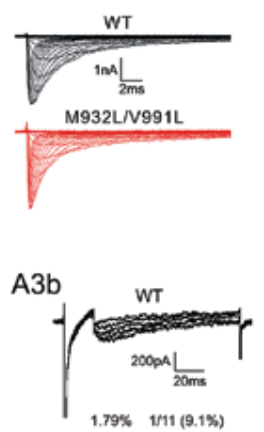

A4

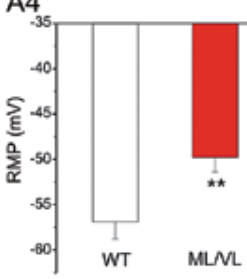

A2
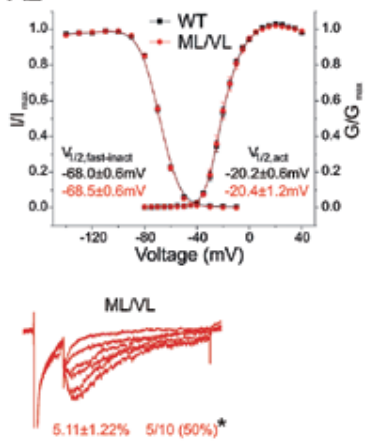

A5

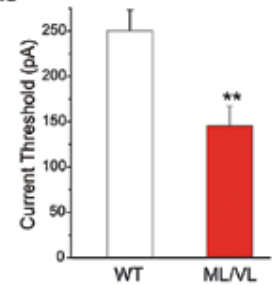

M932L/N991L

A3a

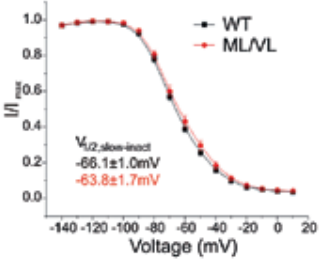

A6

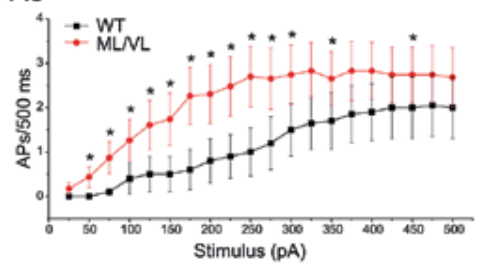

A7

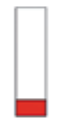

4/27; $15 \%$

(0/20;0\%)

$\Delta=+15 \%(p=0.072)$
Chapter

3.

Legend to figure 5. Electrophysiological analysis of M932L/V991 L mutation. (A) Representative current traces recorded from dorsaroot ganglion (DRG) neurons expressing wild type (WT) (top) or M932L/V991L (bottom) (unless otherwise noted, protocols are the same as in Figs 3 and 4). (B) Activation and steady state fast inactivation for WT (black squares) and M932L/V991L (ML/VL; red circles). Inset shows midpoint values for fast inactivation $\left(\mathrm{V}_{1 / 2, \text { fast-inact }}\right)$ and activation $\left(\mathrm{V}_{1 / 2, \text { act }}\right)$ of WT (black) and M932L/V991L (red), respectively. (C) Steady state slow inactivation of WT (black squares) and M932L/V991 L (red circles). Inset: midpoint values of slow-inactivation ( $\left.\mathrm{V}_{1 / 2 \text {, slow-inact }}\right)$ (WT: black; M932L/V991L: red). (D) Resurgent currents recorded from DRG neuronsexpressing WT (left) or M932L/V991L (right). Resurgent currents were assessed with a 2-step protocol that initially depolarized the membrane to $-30 \mathrm{mV}$ for 20 milliseconds before testing for resurgent sodium currents by hyperpolarizing the membrane potential in $-5 \mathrm{mV}$ increments from $\mathrm{o}$ to $-80 \mathrm{mV}$ for 100 milliseconds, then returning to the holding potential of -100mV. Current amplitude (normalized to peak current evoked by a 130mV depolarization) (left) and proportion of cells producing resurgent current (right) are shown below traces; $* p<0.05$. (E) Resting membrane potential (RMP) of DRG neurons expressing WT $(-56.9 \pm 1.9 \mathrm{mV}, n=20)$ or M932L/V991L $(-49.8 \pm$ 1.6mV, $n=23) ; * * \mathrm{k}<0.01$. (F) Current threshold of DRG neurons expressing WT (250 $\pm 23 \mathrm{pA}, \mathrm{n}=$ 20) or M932L/V991L (145 $\pm 22 \mathrm{pA}, \mathrm{n}=23$ ) to 200-millisecond stimuli; $* * \mathrm{p}<0.01$. (G) Comparison of mean firing frequencies of DRG neurons expressing WT and M $932 \mathrm{~L} / \mathrm{V} 991 \mathrm{~L}$ across the range of current injections from 25 to 500pA; $* p<0.05$. $(H)$ Bar graph showing the proportion of spontaneous firing cells for DRG neurons expressing M932L/V991 (red); numbers to the right of the bar graph show mean values for WT (lower value in parentheses) and M932L/V991 L (upper value); $p=0.072$. The recording on the right shows spontaneous firing (10 seconds) of representative DRG neuron expressing M932L/V991 L; the numbers above the trace show average \pm standard deviation frequency of spontaneous action potentials. $V_{1 / 2}$ represents voltage midpoint, I/I represents normalized current, and G/C represents normalized conductance for fast-activation, slow-inactivation, and activation. $\mathrm{APs}=$ action potentials. 


\section{Discussion}

Despite careful clinical assessment, an underlying cause cannot be found in a substantial number (24 to $>90 \%$ in different series) of patients with SFN.5, 6, 9 In this study we show, in 8 of 28 (28.6\%) patients with skin biopsy and QSTconfirmed I-SFN, missense mutations in the $\mathrm{SCN}_{9} A$ gene, which encodes a voltage-gated sodium channel, $\mathrm{Na}_{\mathrm{v}} \mathrm{T} .7$, that is present within small peripheral nerve fibers. Electrophysiological analysis demonstrated gain-of-function changes in the mutant channels and showed that the mutations share the common feature of rendering DRG neurons hyperexcitable.

Our findings of gain-of-function mutations of $\mathrm{Na}_{\mathrm{v}} 1.7$ in $28.6 \%$ of patients with I-SFN are based on an analysis of 28 Dutch Caucasian patients who met criteria that included no history or detection on screening of disorders known to cause SFN, and confirmation of the diagnosis of SFN by abnormal QST, and by reduced IENFD on skin biopsy. These patients were derived from a larger group of 248 patients referred with a clinical diagnosis of SFN for evaluation at an academic medical center. Aside from any selection bias inherent in referral to an academic medical center, and from any bias introduced by our inclusion/exclusion criteria, which yielded a study cohort of 28 patients meeting stringent criteria for I-SFN, we believe that our sample may be representative of the general Dutch Caucasian population of patients with SFN. Although there were no other distinguishing clinical characteristics, age of onset of symptoms was younger (although not statistically significant) for patients with $S C N_{9} A$ mutations than for patients without $\mathrm{SCNgA}$ mutations.

Mutations in the $S C N$ gA gene have been previously linked to IEM, a rare inherited disorder characterized by distal burning pain, ${ }^{15}$ and PEPD, characterized by perineal, periocular, and perimandibular pain. ${ }^{17}$ Some of our patients with SFN also reported burning feet and hands, or pain around the eyes and jaw. However, despite this apparent similarity, our patients exhibited clinical characteristics typical for small fiber neuropathy ${ }^{1-6}$ and differed from patients with prototypical IEM and PEPD in multiple ways: (1) Autonomic dysfunction is common in SFN, and severe autonomic symptoms were seen in almost all of our patients. Except for skin reddening, autonomic symptoms are not prominent in IEM. ${ }^{5,}{ }^{36}$ (2) Location and onset of pain and related complaints were distributed throughout the body in our patients with I-SFN, whereas in IEM pain is mainly located in the distal extremities. In patient 3 (D623N), painful muscles from early childhood preceded distal complaints, whereas patient 5 ( $11720 \mathrm{~K})$ experienced initial pain throughout the entire body, and patient $8(\mathrm{I} 228 \mathrm{M})$ initially experienced severe jaw pain. (3) Whereas IEM is characterized by erythema of the involved areas, ${ }^{15}$ half of our patients did not display this sign. (4) Our patients did not display the aggravation of symptoms by warmth and relief by cold that are characteristic of 
IEM. ${ }^{15,37}$ Five of our 8 patients denied aggravation by warmth, and 7 had no relief by cold. Patient 8 reported that cold increased symptoms and warmth relieved them. (5) The $\mathrm{Na}_{\mathrm{v}} 1.7$ mutations that we profiled did not display the hyperpolarized activation and enhanced ramp responses characteristic of IEM mutations ${ }^{15}$ or the incomplete fast inactivation ${ }^{17}$ characteristic of PEPD mutations. The present results demonstrate that $\mathrm{Na}_{\mathrm{v}} \mathrm{T} .7$ mutations, distinct from those that have been associated with IEM ${ }^{15}$ and PEPD, ${ }^{17}$ occur in a substantial proportion of patients with I-SFN.

$\mathrm{Na}_{\mathrm{v}} 1.7$ is preferentially expressed within DRG and sympathetic ganglion neurons ${ }^{11,12}$ and their axons, including small diameter $(<0.5 \mu \mathrm{m})$ intracutaneous axon terminals, where it is coexpressed with other sodium channel subtypes $\left(\mathrm{Na}_{\mathrm{v}}{ }^{1} .6 / \mathrm{Na}_{\mathrm{v}} 1.8 / \mathrm{Na}_{\mathrm{v}}{ }^{1.9}\right)$ and the sodium-calcium exchanger $\mathrm{NCX} .{ }^{13} \mathrm{Na}_{\mathrm{v}}{ }^{1.7}$ channels modulate the excitability of these neurons by opening and producing a $\mathrm{Na}^{+}$current in response to small depolarizations close to resting potential, thus bringing the neuron closer to the activation potential of other sodium channel isoforms. ${ }^{14}$ The $\mathrm{Na}_{\mathrm{v}} 1.7$ mutations that we found in patients with SFN impaired slow inactivation, depolarized fast and slow inactivation, or enhanced resurgent currents. Each of the mutations rendered DRG neurons hyperexcitable.

Sodium channel activity has been shown to trigger axonal degeneration via calcium-importing reverse sodium-calcium exchange in axons under conditions where the ability to extrude sodium is exceeded..$^{38,39}$ Degeneration of nonmyelinated axons has been described in hypoxic neuropathy, ${ }^{40}$ and the distal pains reported by some of our patients are similar to the acral paresthesias that have been linked to low $\mathrm{Na} / \mathrm{K}$ adenosine triphosphatase levels in peripheral nerves in chronic mountain sickness. ${ }^{4}$ Although there is no reason to believe that the patients we have described suffered from systemic hypoxia, $\mathrm{Na}^{+}$influx is known to impose an energetic load on neurons and neuronal processes, ${ }^{42}$ and increased activity of mutant $\mathrm{Na}_{\mathrm{v}} \mathrm{T} .7$ channels would be expected to have an especially large effect on small diameter intracutaneous axons, where NCX is present, ${ }^{13}$ due to their high surface to volume ratio and input resistance, low capacitance per unit length, and shorter wavelength. ${ }^{43,44}$ Consistent with a role of sodium channels in I-SFN, action potential activity at physiological frequencies can sensitize axons to otherwise reversible metabolic insults, and can produce degeneration of these axons ${ }^{45}$ that is attenuated by sodium channel blockers..$^{46}$ In conclusion, we demonstrate the occurrence of missense mutations in the $\mathrm{SCN}_{9} \mathrm{~A}$ gene encoding the $\mathrm{Na}_{\mathrm{V}} \mathrm{T} .7$ sodium channel, in a substantial proportion $(28.6 \%)$ of patients with biopsy- and QST-confirmed I-SFN, and show that these mutations render DRG neurons that give rise to small axons hyperexcitable. Expression of $\mathrm{Na}_{\mathrm{v}} 1.7$ and $\mathrm{NCX}$ in small diameter axons may cause these fibers to degenerate in response to gain-of-function changes produced by $\mathrm{Na}_{\mathrm{v}} \mathrm{T} .7$ mutations such as those described in this paper. Our results suggest 
that these mutations may predispose to the development of channelopathyassociated SFN. SCNgA gene analysis might be considered for patients with SFN in whom other causes are excluded, particularly patients with younger ages of onset. In terms of treatment, existing nonspecific sodium channel blockers, $\mathrm{Na}_{\mathrm{v}}$ 1.7-selective blockers when available, and inhibitors of $\mathrm{NCX} 2$ merit study as therapeutic approaches that might slow or halt axonal degeneration in I-SFN. 


\section{References}

1. Holland, N.R. et al. Small-fiber sensory neuropathies: clinical course and neuropathology of idiopathic cases. Ann Neurol 44, 47-59 (1998).

2. Gorson, K.C. \& Ropper, A.H. Idiopathic distal small fiber neuropathy. Acta Neurol Scand 92, 376-82 (1995).

3. Stewart, J.D., Low, P.A. \& Fealey, R.D. Distal small fiber neuropathy: results of tests of sweating and autonomic cardiovascular reflexes. Muscle Nerve 15, 661-5 (1992).

4. Low, P.A. Clinical autonomic disorders: evaluation and management. (Lippincott-Raven, Philadelphia, 1997).

5. Lacomis, D. Small-fiber neuropathy. Muscle Nerve 26, 173-88 (2002).

6. Devigili, G. et al. The diagnostic criteria for small fibre neuropathy: from symptoms to neuropathology. Brain 131, 1912-25 (2008).

7. Tesfaye, S. et al. Diabetic neuropathies: update on definitions, diagnostic criteria, estimation of severity, and treatments. Diabetes Care 33, 2285-93 (2010).

8. Lauria, G. Small fibre neuropathies. Curr Opin Neurol 18, $591-7$ (2005).

9. Bednarik, J. et al. Etiology of small-fiber neuropathy. J Peripher Nerv Syst 14, 177-83 (2009).

10. Stogbauer, F. et al. Autosomal dominant burning feet syndrome.J Neurol Neurosurg Psychiatry 67, 78-81 (1999).

11. Toledo-Aral, J.J. et al. Identification of $\mathrm{PN} 1$, a predominant voltage-dependent sodium channel expressed principally in peripheral neurons. Proc Natl Acad Sci U S A 94, 1527-32 (1997).

12. Rush, A.M. et al. A single sodium channel mutation produces hyper- or hypoexcitability in different types of neurons. Proc Natl Acad Sci U S A 103, 8245-50 (2006).

13. Persson, A.K. et al. Sodium-calcium exchanger and multiple sodium channel isoforms in intra-epidermal nerve terminals. Mol Pain 6, 84 (2010).

14. Cummins, T.R., Howe, J.R. \& Waxman, S.G. Slow closed-state inactivation: a novel mechanism underlying ramp currents in cells expressing the hNE/PN1 sodium channel. J Neurosci 18, 9607-19 (1998).

15. Dib-Haji, S.D., Cummins, T.R., Black, J.A. \& Waxman, S.G. Sodium channels in normal and pathological pain. Annu Rev Neurosci 33, 325-47 (2010).

16. Dib-Hajj, S.D. et al. Gain-of-function mutation in Nav1.7 in familial erythromelalgia induces bursting of sensory neurons. Brain 128, 1847-54 (2005).

17. Fertleman, C.R. et al. SCNgA mutations in paroxysmal extreme pain disorder: allelic variants underlie distinct channel defects and phenotypes. Neuron 52, 767-74 (2006).

18. Cox, J.J. et al. An SCNgA channelopathy causes congenital inability to experience pain. Nature 444, 894-8 (2006).

19. Reilly, M.M. Sorting out the inherited neuropathies. Pract Neurol 7, 93-105 (2007).

20. Chamberlain, J.L. et al. Peripherin-IgG association with neurologic and endocrine autoimmunity. J Autoimmun 34, 469-77 (2010).

21. Lauria, G. et al. European Federation of Neurological Societies/Peripheral Nerve Society Guideline on the use of skin biopsy in the diagnosis of small fiber neuropathy. Report of a joint task force of the European Federation of Neurological Societies and the Peripheral Nerve Society. Eur J Neurol 17, 903-12, e44-9 (2010).

22. Bakkers, M. et al. Intraepidermal nerve fiber density and its application in sarcoidosis. Neurology 73, 11428 (2009).

23. Shy, M.E. et al. Quantitative sensory testing: report of the Therapeutics and Technology Assessment Subcommittee of the American Academy of Neurology. Neurology 60, 898-904 (2003).

24. Reulen, J.P., Lansbergen, M.D., Verstraete, E. \& Spaans, F. Comparison of thermal threshold tests to assess small nerve fiber function: limits vs. levels. Clin Neurophysiol 114, 556-63 (2003).

25. Yarnitsky, D. \& Sprecher, E. Thermal testing: normative data and repeatability for various test algorithms. J Neurol Sci 125, 39-45 (1994).

26. Hoitsma, E. et al. Abnormal warm and cold sensation thresholds suggestive of small-fibre neuropathy in sarcoidosis. Clin Neurophysiol 114, 2326-33 (2003).

27. Suarez, G.A. et al. The Autonomic Symptom Profile: a new instrument to assess autonomic symptoms. Neurology 52, 523-8 (1999). 
28. Galer, B.S. \& Jensen, M.P. Development and preliminary validation of a pain measure specific to neuropathic pain: the Neuropathic Pain Scale. Neurology 48, 332-8 (1997).

29. Maxwell, C. Sensitivity and accuracy of the visual analogue scale: a psycho-physical classroom experiment. BrJ Clin Pharmacol 6, 15-24 (1978).

30. Drenth, J.P. et al. $\mathrm{SCN} g \mathrm{~A}$ mutations define primary erythermalgia as a neuropathic disorder of voltage gated sodium channels. J Invest Dermatol 124, 1333-8 (2005).

31. Klugbauer, N., Lacinova, L., Flockerzi, V. \& Hofmann, F. Structure and functional expression of a new member of the tetrodotoxin-sensitive voltage-activated sodium channel family from human neuroendocrine cells. EMBOJ 14, 1084-90 (1995).

32. Han, C. et al. Early- and late-onset inherited erythromelalgia: genotype-phenotype correlation. Brain $\mathbf{1 3 2}$ 1711-22 (2009).

33. Cummins, T.R., Rush, A.M., Estacion, M., Dib-Hajj, S.D. \& Waxman, S.G. Voltage-clamp and currentclamp recordings from mammalian DRG neurons. Nat Protoc 4, 1103-12 (2009).

34. Dib-Hajj, S.D. et al. Transfection of rat or mouse neurons by biolistics or electroporation. Nat Protoc 4 1118-26 (2009).

35. Jarecki, B.W., Piekarz, A.D., Jackson, J.O., 2nd \& Cummins, T.R. Human voltage-gated sodium channel mutations that cause inherited neuronal and muscle channelopathies increase resurgent sodium currents. J Clin Invest 120, 369-78 (2010).

36. Drenth, J.P. \& Waxman, S.G. Mutations in sodium-channel gene SCNgA cause a spectrum of human genetic pain disorders. J Clin Invest 117, 3603-9 (2007).

37. Michiels, J.J., te Morsche, R.H., Jansen, J.B. \& Drenth, J.P. Autosomal dominant erythermalgia associated with a novel mutation in the voltage-gated sodium channel alpha subunit Nav1.7. Arch Neurol 62, 1587-90 (2005).

38. Stys, P.K., Waxman, S.G. \& Ransom, B.R. Na(+)-Ca2+ exchanger mediates Ca2+ influx during anoxia in mammalian central nervous system white matter. Ann Neurol 30, 375-80 (1991).

39. Garthwaite, G., Goodwin, D.A., Batchelor, A.M., Leeming, K. \& Garthwaite, J. Nitric oxide toxicity in CNS white matter: an in vitro study using rat optic nerve. Neuroscience 109, 145-55 (2002).

40. Malik, R.A. et al. Hypoxic neuropathy: relevance to human diabetic neuropathy. Diabetologia $33,311-8$ (1990).

41. Appenzeller, O. et al. Acral paresthesias in the Andes and neurology at sea level. Neurology 59, 1532-5 (2002)

42. Ames, A., 3rd. CNS energy metabolism as related to function. Brain Res Brain Res Rev 34, 42-68 (2000).

43. Waxman, S.G., Black, J.A., Kocsis, J.D. \& Ritchie, J.M. Low density of sodium channels supports action potential conduction in axons of neonatal rat optic nerve. Proc Natl Acad Sci U S A 86, 1406-10 (1989).

44. Donnelly, D.F. Spontaneous action potential generation due to persistent sodium channel currents in simulated carotid body afferent fibers. J Appl Physiol 104, 1394-401 (2008).

45. Smith, K.J., Kapoor, R., Hall, S.M. \& Davies, M. Electrically active axons degenerate when exposed to nitric oxide. Ann Neurol 49, 470-6 (2001).

46. Kapoor, R., Davies, M., Blaker, P.A., Hall, S.M. \& Smith, K.J. Blockers of sodium and calcium entry protect axons from nitric oxide-mediated degeneration. Ann Neurol 53, 174-80 (2003). 



\section{Chapter 4}

\section{Intra- and interfamily phenotypic diversity in}

pain syndromes associated with a gain-of-function variant of $\mathrm{Na}_{\mathrm{v}} 1.7$

M. Estacion ${ }^{a, b * *, C . ~ H a n}{ }^{a, b * *}$, J.S. Choi ${ }^{a, b, c * *}$, J.G.J. Hoeijmakers ${ }^{d}$, G. Lauria ${ }^{e}$, J.P.H. Drenthf, M.M. Gerrits, S.D. Dib-Hajj ${ }^{a, b}$, C.G. Faber ${ }^{d}$, I.S.J. Merkies ${ }^{d, h}$, Stephen G Waxman ${ }^{a, b}$.

* co-first authorship

aDepartment of Neurology, Yale University School of Medicine, New Haven, CT, USA ${ }^{b}$ Center for Neuroscience and Regeneration Research, Veterans Affairs Medical Center,

West Haven, CT, USA

'College of Pharmacy, Catholic University of Korea, Bucheon, South Korea ${ }^{\mathrm{d} D e p a r t m e n t}$ of Neurology, Maastricht University Medical Center, Maastricht eNeuromuscular Diseases Unit, IRCCS Foundation, Carlo Besta Neurological Institute, Milan, Italy

fDepartment of Gastroenterology and Hepatology, Radboud University Nijmegen Medical Center, Nijmegen

${ }^{g}$ Department of Clinical Genomics, Maastricht University Medical Center, Maastricht ${ }^{\text {hDepartment }}$ of Neurology, Spaarne Hospital, Hoofddorp

Molecular Pain. 2011;7:92; published with permission from BioMed Central 


\section{Abstract}

Background: Sodium channel $\mathrm{Na}_{\mathrm{v}} \mathrm{T} .7$ is preferentially expressed within dorsal root ganglia (DRG), trigeminal ganglia and sympathetic ganglion neurons and their fine-diameter axons, where it acts as a threshold channel, amplifying stimuli such as generator potentials in nociceptors. Gain-of-function mutations and variants (single amino acid substitutions) of $\mathrm{Na}_{\mathrm{v}} \mathrm{\gamma} .7$ have been linked to three pain syndromes: Inherited Erythromelalgia (IEM), Paroxysmal Extreme Pain Disorder (PEPD), and Small Fiber Neuropathy (SFN). IEM is characterized clinically by burning pain and redness that is usually focused on the distal extremities, precipitated by mild warmth and relieved by cooling, and is caused by mutations that hyperpolarize activation, slow deactivation, and enhance the channel ramp response. PEPD is characterized by perirectal, periocular or perimandibular pain, often triggered by defecation or lower body stimulation, and is caused by mutations that severely impair fast-inactivation. SFN presents a clinical picture dominated by neuropathic pain and autonomic symptoms; gain-of-function variants have been reported to be present in approximately $30 \%$ of patients with biopsy-confirmed idiopathic SFN, and functional testing has shown altered fast-inactivation, slow-inactivation or resurgent current. In this paper we describe three patients who house the $\mathrm{Na}_{\mathrm{v}} 1.7 / 1228 \mathrm{M}$ variant.

Methods: We have used clinical assessment of patients, quantitative sensory testing and skin biopsy to study these patients, including two siblings in one family, in whom genomic screening demonstrated the $1228 \mathrm{M} \mathrm{Na}_{\mathrm{v}} 1.7$ variant. Electrophysiology (voltage-clamp and current-clamp) was used to test functional effects of the variant channel.

Results: We report three different clinical presentations of the $1228 \mathrm{M} \mathrm{Na}_{\mathrm{v}} 1.7$ variant: presentation with severe facial pain, presentation with distal (feet, hands) pain, and presentation with scalp discomfort in three patients housing this $\mathrm{Na}_{\mathrm{v}} 1.7$ variant, two of which are from a single family. We also demonstrate that the $\mathrm{Na}_{\mathrm{v}} 1.7 / 228 \mathrm{M}$ variant impairs slow-inactivation, and produces hyperexcitability in both trigeminal ganglion and DRG neurons.

Conclusion: Our results demonstrate intra- and interfamily phenotypic diversity in pain syndromes produced by a gain-of-function variant of $\mathrm{Na}_{\mathrm{v}} 1.7$. 


\section{Introduction}

Sodium channel $\mathrm{Na}_{\mathrm{v}} 1.7$ is preferentially and abundantly expressed within dorsal root ganglia (DRG), ${ }^{1,2}$ trigeminal gangliaa ${ }^{3}$ and sympathetic ganglion neurons, ${ }^{1,2}$ and their fine-diameter axons. ${ }^{4}$ The physiological attributes of $\mathrm{Na}_{\mathrm{v}} 1.7$ include slow closed-state inactivation, which permits activation of the channel in response to small, slow depolarizations close to resting potential. $\mathrm{Na}_{\mathrm{v}}{ }^{1.7}$ thus acts as a threshold channel, amplifying stimuli such as generator potentials in nociceptors, thereby setting their gain. ${ }^{6}$

Gain-of-function mutations and variants (single amino acid substitutions) of $\mathrm{Na}_{\mathrm{v}} \mathrm{T} .7$ have been linked to three pain syndromes. Inherited erythromelalgia (IEM) is characterized clinically by burning pain and redness that is usually focused on the distal extremities, precipitated by mild warmth and relieved by cooling, and is caused by $\mathrm{Na}_{\mathrm{v}} 1.7$ mutations that hyperpolarize activation, slow deactivation, and enhance the channel ramp response.7 Paroxysmal extreme pain disorder (PEPD) is characterized by perirectal, periocular or perimandibular pain, often triggered by defecation or lower body stimulation, ${ }^{8}$ and has been linked to $\mathrm{Na}_{\mathrm{v}} 1.7$ mutations that severely impair fast-inactivation. ${ }^{9}$ Small Fiber Neuropathy (SFN), which involves thinly myelinated and unmyelinated peripheral nerve fibers, ${ }^{10,11}$ presents a clinical picture that is characteristically dominated by neuropathic pain and autonomic symptoms, ${ }^{12}$ together with preservation of normal strength, tendon reflexes, and vibration sense, and normal nerve conduction studies (NCS), which rule out large fiber involvement. The diagnosis of SFN can be confirmed by demonstration of reduced intraepidermal nerve fiber density (IENFD) on skin biopsy and/or abnormal quantitative sensory testing (QST). ${ }^{13,14}$ No apparent cause for SFN can be identified in $24 \%$ to $93 \%$ of cases in published patient series, and these cases are termed idiopathic I-SFN. ${ }^{10,15,16}$ Faber et al., recently reported that gainof-function variants (single amino acid substitutions) of voltage-gated sodium channel $\mathrm{Na}_{\mathrm{v}} 1.7$ are present in approximately $30 \%$ of patients with biopsyconfirmed I-SFN. ${ }^{17}$

Distal (feet, and in some cases, hands) burning or stabbing pain or paraesthesias are the initial symptoms in most patients with I-SFN, and facial pain is rare. Most of the eight patients with SFN described earlier by Faber et al., ${ }^{17}$ fit this clinical picture, and presented with pain in the feet and in some cases the hands early in their course, but did not manifest facial pain. ${ }^{17}$ In contrast, one patient in this series presented with severe pain in the teeth, jaw, and behind the eyes. This patient (patient 8 in Faber et al., 2011) harbored the $\mathrm{Na}_{\mathrm{v}} 1.7$ variant c.684C $>\mathrm{G}(\mathrm{I} 228 \mathrm{M})$; $^{17}$ functional properties of this variant have not been previously reported. We subsequently studied the sister of this patient, who houses the same variant (c.684C $>\mathrm{G}\left(\mathrm{I}_{228 \mathrm{M})}\right.$ in $\left.\mathrm{Na}_{\mathrm{v}} \mathrm{l} \cdot 7\right)$ and suffers from a different 
syndrome of pain and redness of the hands and feet triggered by warmth, and have encountered an additional patient housing the same $\mathrm{Na}_{\mathrm{v}} \mathrm{T}$.7 variant with pain over the scalp. In this study we report these three different clinical presentations of the $1228 \mathrm{M} \mathrm{Na}_{\mathrm{v}} 1.7$ variant, and demonstrate the effects of the $\mathrm{Na}_{\mathrm{v}} 1.7 / 228 \mathrm{M}$ channels on excitability in both trigeminal ganglion and DRG neurons.

\section{Results}

\section{Patient 1}

This patient, described as patient number 8 in Faber et al., (2011) 17 is a 51-yearold male, referred with complaints that started at age 32 , when he experienced excruciating pain in his teeth and jaw triggered by cold and heat, which could radiate to the temporomandibular joint, and pain behind both eyes, especially when looking at bright light. The oral mucosa, lips and tongue were not affected. Multiple tooth extractions did not provide pain relief. He subsequently developed myalgia, with muscle pain persisting for 5-6 days after light physical activity. The pain was aggravated by cold temperature and relieved by warmth. Sometimes the feet were also swollen. This patient suffered from stomach cramps and diarrhea for more than 35 years, and from dry mouth and eyes and reduced urinary sensation and intermittent hesitation for several years. The patient was severely disabled and unable to work due to these complaints. Acetaminophen made the pain bearable, while short trials of NSAIDs and antidepressants did not provide relief. Physical examination showed no abnormalities. Laboratory investigations, nerve conduction studies and chest X-ray were normal. IENFD $\left(1.6 / \mathrm{mm}\right.$; age- and gender-matched normal values $\left.\geq 3.5 / \mathrm{mm}^{18}\right)$ was abnormal. QST revealed abnormal warm and cold thresholds of the right foot. SCNgA gene analyses demonstrated the variant, c.684C $>\mathrm{G} ; \mathrm{Na}_{\mathrm{v}} 1.7 / \mathrm{l} 228 \mathrm{M}$. The patient was diagnosed with $\mathrm{Na}_{\mathrm{v}}$ 1.7-related SFN. The patient's two sons, aged 27 and 29, were found to house the $1228 \mathrm{M}$ substitution, but did not have any complaints at the time of study.

The I228M variant substitutes a highly conserved residue near the C-terminus of the $\mathrm{S}_{4}$ segment in domain I (DI/S4, Figure 1). All human sodium channels except $\mathrm{Na}_{\mathrm{v}} 1.9$ carry an isoleucine at this position, ${ }^{19}$ and this residue is invariant in all $\mathrm{Na}_{\mathrm{v}} 1.7$ orthologues from mammalian species (data not shown). The conservation of the 1228 residue among sodium channels suggests that the I228M substitution might alter the properties of the $\mathrm{Na}_{\mathrm{v}} 1.7$ channels (Figure 1). This substitution was not found in a control panel of DNA from 100 healthy Dutch (Caucasian) individuals (200 chromosomes). However, I228M is listed as a natural SNP in one database (Craig Venter Human Genome), but with no 
breakdown of major/minor allele frequency, and has been reported as being associated with Dravet syndrome $\mathrm{e}^{20}$ and in $<0.3 \%$ of a control population $(5 / 576$ control chromosomes).

Figure 1. Schematic of I228M mutation

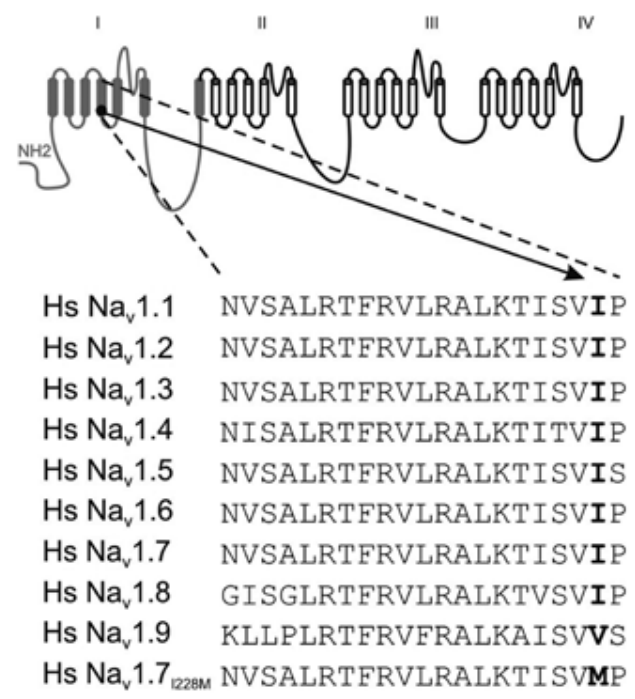

Legend to figure 1. Sequence alignment of $\mathrm{DI} / \mathrm{S}_{4}$ from human sodium channels. The chargeconserved substitution in DI/S4 replaces a highly conserved isoleucine residue at the cytoplasmic end of the $\mathrm{S}_{4}$ helix. $\mathrm{I}_{228}$ is conserved in all human sodium channels except for $\mathrm{Na}_{\mathrm{v}} 1.9$ which has a conservative substitution, valine, at the corresponding position.

\section{Patient 2}

This patient, who is the sister of Patient 1 , gave a history of burning pain and redness of hands and feet, triggered by rising temperature and exercise and relieved by cooling, beginning at age 36 years. She also reported increased perspiration, gastrointestinal complaints and hot flashes. Her medical history revealed recurrent urticaria attacks, psoriatic arthritis and hypothyroidism for which she is adequately treated. Physical examination showed no abnormalities other than red discolored hands. Laboratory investigations, nerve conduction studies and chest X-ray were normal. Quantitative sensory testing showed no abnormalities. IENFD was 8 per $\mathrm{mm}$ (normal values $\geq 5.7$ per $\mathrm{mm}$ ). ${ }^{18}$ DNA analysis showed the same substitution in the $S C N g A$ gene as found in her brother. On the basis of the clinical history and findings, the patient was diagnosed as having probable SFN. 


\section{Patient 3}

This 46-year-old woman presented with a red discoloration of the occiput. Three months later the red area expanded and was noted to be associated with a tingling, burning and warm sensation over the scalp. After washing of the hair, the redness increased for one hour. The patient also complained that the structure of her hair changed, becoming dryer and more fragile. She noted improvement in these complaints with warm temperatures (such as during a visit to the Caribbean) and with fever. Cold had no specific influence. A year following onset of scalp symptoms, a red discoloration of the toes of both feet developed, together with paraesthesias and a burning sensation and tingling in both hands. In addition, the patient reported severe perspiration since puberty and intermittent difficulties with micturition. No other dysautonomic symptoms were noted. Ibuprofen did not relieve the pain. The family history was negative. Neurological examination was unremarkable. Laboratory investigations, a chest $X$-ray and nerve conduction studies were normal. Quantitative sensory testing showed abnormal thresholds for warmth and cold sensation of the dorsum of the right foot. A skin biopsy demonstrated an IENFD of 5.2 per $\mathrm{mm}$, which was lower than the reported normative values $(\leq 5.7 / \mathrm{mm}) .{ }^{18}$ The patient was diagnosed as having I-SFN. SCN9A gene analysis demonstrated the same variant, c.684C > G; p. I228M, as in Patients 1 and 2.

\section{Functional Analysis}

Voltage-clamp analysis

Voltage-clamp analysis of $1228 \mathrm{M}$ variant channels following expression in HEK293 cells (Figure 2) demonstrated impaired slow-inactivation (Figure 2D). Current densities (WT: $432 \pm 90 \mathrm{pA} / \mathrm{pF}, \mathrm{n}=9$; I228M: $357 \pm 70 \mathrm{pA} / \mathrm{pF} \mathrm{n}=$ 13), activation $\mathrm{V}_{1 / 2}(\mathrm{WT}:-26.1 \pm 2.5 \mathrm{mV}, \mathrm{n}=9$; $\mathbf{2} 28 \mathrm{M}:-25.3 \pm 1.0 \mathrm{mV}, \mathrm{n}=13$ ), and fast-inactivation $\mathrm{V}_{1} / 2$ (WT: $-81.2 \pm 2.2 \mathrm{mV}, \mathrm{n}=8$; I $228 \mathrm{M}:-83.1 \pm 1.2 \mathrm{mV}$, $n=12$ ), for HEK293 cells transfected with WT or $1228 \mathrm{M}$ channels were not significantly different (Figure 2A, B, C). The time constants for fast-inactivation (Figure $2 \mathrm{E}$ ) and deactivation (Figure $2 \mathrm{~F}$ ) were not significantly different for $1228 \mathrm{M}$ versus WT channels. Persistent current (non-inactivating component at $0 \mathrm{mV}$ ), measured in CsF-based pipette solution (WT: $0.42 \% \pm 0.12 \%, n=8$; I228M: $0.67 \% \pm 0.20 \%, n=12$ ) and in aspartate-based pipette solution (WT: $0.41 \% \pm 0.08 \%, n=14 ; \mid 228 \mathrm{M}: 0.41 \% \pm 0.15 \%, n=13$ ) were not significantly different for $1228 \mathrm{M}$ versus wild-type channels. Slow-inactivation was impaired for $1228 \mathrm{M}$ channels (Figure $2 \mathrm{D}$ ), with a depolarized $\mathrm{V}_{1} / 2$ (WT: $-63.0 \pm 1.8 \mathrm{mV}$, $\mathrm{n}=10 ;$ I $228 \mathrm{M}:-56.2 \pm 1.2 \mathrm{mV}, \mathrm{n}=14 ; \mathrm{p}<0.05)$. The offset for slow-inactivation (non-inactivating component at $10 \mathrm{mV}$ ) was not significantly larger for $1228 \mathrm{M}$ compared to wild-type channels (WT: $7.8 \% \pm 1.3 \%, n=10$; $1228 \mathrm{M}: 7.8 \% \pm 1.3 \%$, 


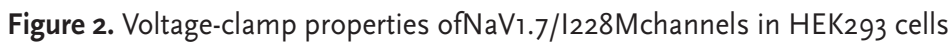

A

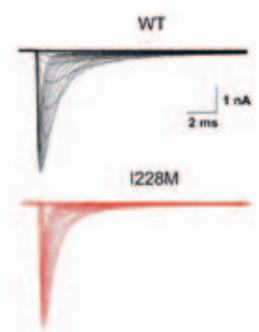

C

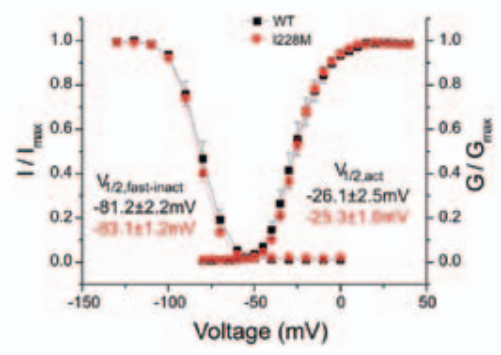

E

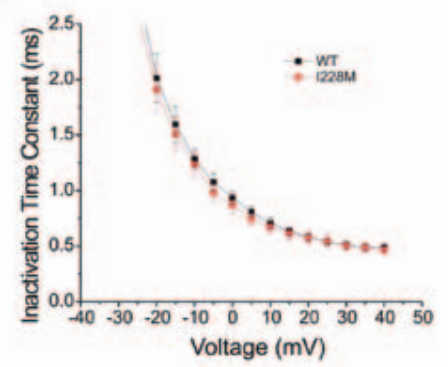

B

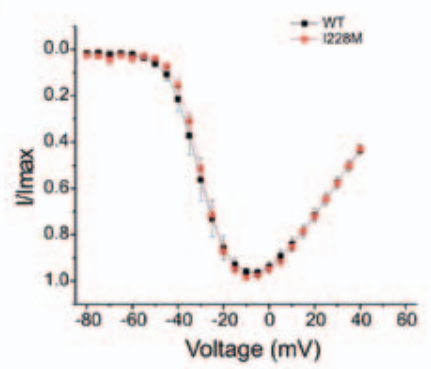

D

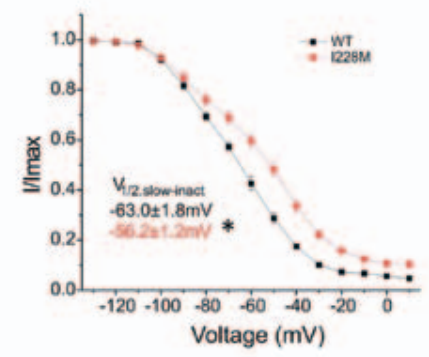

$\mathrm{F}$

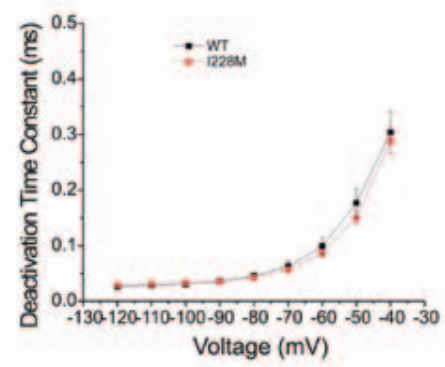

Legend to figure 2. Electrophysiological analysis of $1228 \mathrm{M}$ variant: (A) Representative current traces recorded from HEK293 cells expressing wild type $\mathrm{Na}_{\mathrm{v}} 1.7$ (WT) (top) or $1228 \mathrm{M}$ (bottom) channels, evoked by voltage steps (100 mec) from -80 to $+40 \mathrm{mV}$ in $5 \mathrm{mV}$ increments, from a holding potential of -120 mV. (B) Normalized I-V curves for WT and I228M expressing cells. (C) Activation and steady-state fast-inactivation for WT (black squares) and I228M (red circles). Fastinactivation was examined using a series of $500 \mathrm{msec}$ prepulses from -140 to o $\mathrm{mV}$ followed by test pulses to -10 mV. Left inset: midpoint values for fast inactivation $\left(\mathrm{V}_{1 / 2, \text { fast-inact }}\right)$ of WT (black) and I228M (red). Right inset: midpoint values for activation ( $\mathrm{V}_{1 / 2}$, act) of WT (black) and I228M (red). (D) Steady-state slow inactivation of WT (black squares) and I228M (red circles). Slow-inactivation was assessed using a $20 \mathrm{msec}$ pulse to $-10 \mathrm{mV}$ after a 30 second prepulse to potentials from -130 to $10 \mathrm{mV}$ followed by a $100 \mathrm{msec}$ pulse to $-120 \mathrm{mV}$ to remove fast-inactivation. Inset: midpoint values of slow inactivation ( $\mathrm{V}_{1 / 2 \text {, slow-inact) }}$ (WT: black; I228M: red); $^{*} \mathrm{p}<0.05 . \mathrm{V}_{1 / 2}$ represents voltage midpoint, $1 / I_{\max }$ represents normalized current, and $\mathrm{G} / \mathrm{G}_{\max }$ represents normalized conductance for fast-activation, slow-inactivation, and activation. (E) The kinetics of inactivation were analyzed by fitting data with a single exponential function for WT and I228M currents. (F) The kinetics of deactivation for WT and $1228 \mathrm{M}$ expressing cells were obtained by holding the cells at $-120 \mathrm{mV}$ and tail currents were generated by a brief $0.5 \mathrm{~ms}$ depolarization to $-20 \mathrm{mV}$ followed by a series of repolarizations ranging from -120 to $-40 \mathrm{mV}$. The closing rate of the channels was obtained by fitting the tail currents with a single exponential function. 
$n=14)$. Impaired slow-inactivation would be expected to increase the number of channels available for activation at potentials positive to $-100 \mathrm{mV}$, including potentials close to resting potential of DRG neurons.

\section{Current-Clamp Analysis: DRG Neurons}

I228M had strong functional effects on DRG neurons, which were clearly rendered hyperexcitable by these channels (Figure 3). $1228 \mathrm{M}$ produced a 4.8 $\mathrm{mV}$ depolarizing shift in resting membrane potential of transfected neurons (WT: $-58.5 \pm 1.4 \mathrm{mV}, \mathrm{n}=22$; I228M: $-53.7 \pm 1.7 \mathrm{mV}, \mathrm{n}=12 ; \mathrm{p}<0.05$ ). While I228M

Figure 3. Current-clamp properties of DRG neurons transfected with I228M

A

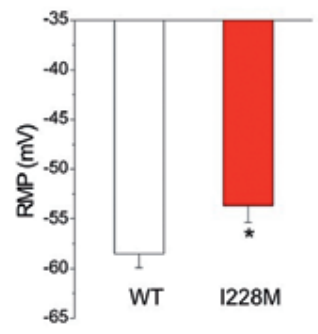

B

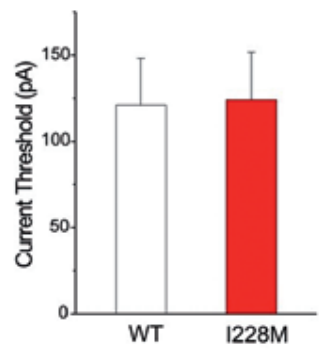

$\mathrm{C}$

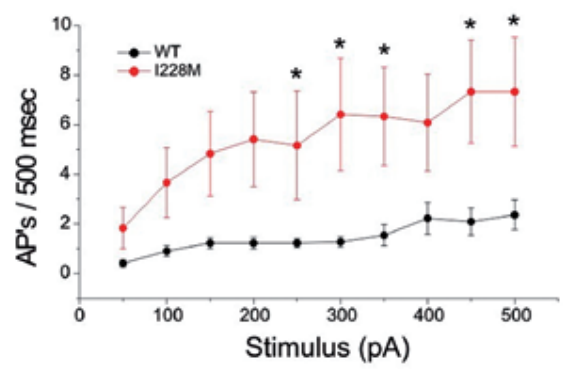

D
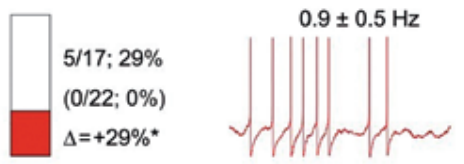

Legend to figure 3. Excitability of DRG neurons expressing 1228M: (A) RMP of DRG neurons expressing WT $(-58.5 \pm 1.4, n=22)$ or $1228 \mathrm{M}(-53.7 \pm 1.7, n=12)$; * $p<0.05$. (B) Current threshold of DRG neurons expressing WT $(121 \pm 27, n=22)$ or $1228 \mathrm{M}(124 \pm 28, n=12)$ to 200 msec stimuli. (C) Comparison of mean firing frequency in DRG neurons expressing WT and I228M across a range of current injections from 50 to 500pA; *p < 0.05. (D) Bar graph showing the proportion of spontaneous firing cells for DRG neurons expressing $1228 \mathrm{M}$ (red) and WT channels (black); numbers to the right of the bar graph show values for WT (lower value in parentheses) and I228M (upper value); $* p<0.05$. The recording on the right shows spontaneous firing (10 seconds) of representative DRG neuron expressing $1228 \mathrm{M}$; the numbers above the trace show average \pm standard deviation frequency of spontaneous action potentials. APs = action potentials. 
did not decrease the current threshold (WT: $121 \pm 27$ pA, $n=22$; I228M: $124 \pm$ $28 \mathrm{pA}, \mathrm{n}=12$ ), it produced on average a higher firing frequency at all stimulus intensities, even close to current threshold, and increased the number of action potentials evoked by 500-millisecond depolarizing stimuli at higher stimulus intensities nearly four-fold, with the change being statistically significant at many

Figure 4. Current-clamp properties of trigeminal ganglion neurons transfected with $1228 \mathrm{M}$

A

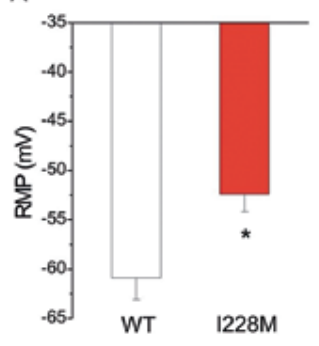

C

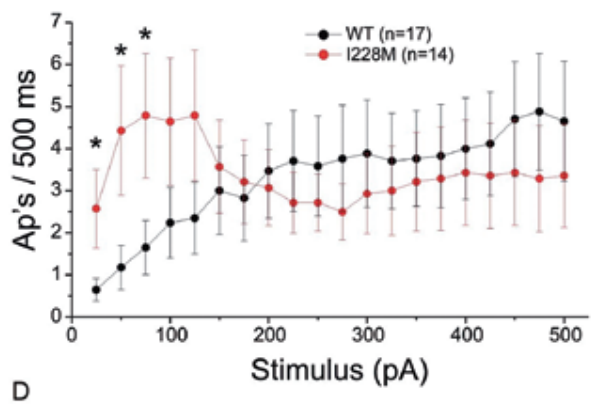

B

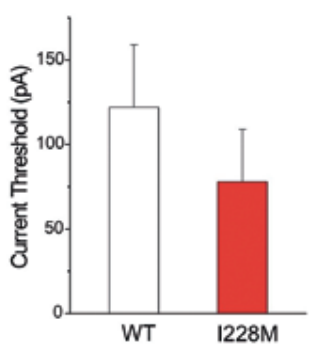

Chapter

4.

Legend to figure 4. Excitability of trigeminal ganglion neurons expressing $1228 \mathrm{M}$ : (A) RMP of trigeminal ganglion neurons expressing WT $(-60.9 \pm 2.2, n=17)$ or $1228 \mathrm{M}(-52.4 \pm 1.8, n=14)$; $* \mathrm{p}<0.05$. (B) Current threshold of DRG neurons expressing WT $(122 \pm 37, \mathrm{n}=13)$ or $1228 \mathrm{M}$ $(78 \pm 31, n=12)$ to 200 msec stimuli; $p<0.05$. (C) Comparison of mean firing frequency in trigeminal ganglion neurons expressing WT and 2228 $_{2}$ across a range of current injections from 25 to $500 \mathrm{pA} ; * \mathrm{p}<0.05$. (D) Bar graph showing the proportion of spontaneous firing cells for trigeminal neurons expressing I228M (red) and WT channels (black); numbers to the right of the bar graph show mean values for WT (lower value in parentheses) and I228M (upper value). The recording on the right shows spontaneous firing (10 seconds) of representative trigeminal neuron expressing $1228 \mathrm{M}$; the numbers above the trace show average \pm standard deviation frequency of spontaneous action potentials. APs $=$ action potentials. 
of the intensities tested, from 50 to $500 \mathrm{pA}$. $1228 \mathrm{M}$ also produced a significant increase in the proportion of spontaneously firing cells (5 of 17 [29\%] vs $O$ of $22[0 \%]$ for cells transfected with WT channels) $(p<0.05)$; mean frequency of spontaneous activity in cells transfected with $1228 \mathrm{M}$ was $0.9 \pm 0.5 \mathrm{~Hz}(n=5)$.

\section{Current Clamp Analysis: Trigeminal Ganglion Neurons}

The $1228 \mathrm{M}$ variant produced hyperexcitability in trigeminal ganglion neurons (Figure 4). $1228 \mathrm{M}$ produced an $8.5 \mathrm{mV}$ depolarizing shift in resting membrane potential (WT: $-60.9 \pm 2.2 \mathrm{mV}, \mathrm{n}=17$; $1228 \mathrm{M}:-52.4 \pm 1.8 \mathrm{mV}, \mathrm{n}=14 ; \mathrm{p}<0.05$ ). $1228 \mathrm{M}$ produced a $36 \%$ reduction in current threshold to 200 -millisecond stimuli (WT: $122 \pm 37 \mathrm{pA}, \mathrm{n}=13$; I228M: $78 \pm 31 \mathrm{pA}, \mathrm{n}=12$ ). Trigeminal ganglion neurons transfected with $1228 \mathrm{M}$ tended to fire multiple action potentials (at a frequency nearly four-fold higher than in cells transfected with wild-type channels) in response to 500 msec stimuli close to threshold (25 to $125 \mathrm{pA}$, with the difference being statistically significant between 25 and $75 \mathrm{pA}$ ), although at stimulus levels of $\geq 2 \mathrm{X}$ threshold, the number of action potentials falls, approaching that of cells transfected with wild-type channels (Figure 4C). I228M produced a trend toward an increase in the proportion of spontaneously firing cells ( 4 of 18 [22\%] vs 3 of 20 [15\%] for cells transfected with WT channels) that did not reach statistical significance; mean frequency of spontaneous activity in cells transfected with $1228 \mathrm{M}$ was $0.2 \pm 0.05 \mathrm{~Hz}(n=4)$.

\section{Discussion}

In this study we describe three patients (two siblings, and a third, unrelated patient) housing the $1228 \mathrm{M}$ variant of sodium channel $\mathrm{Na}_{\mathrm{v}} 1.7$. One of these patients displayed a clinical phenotype that included pain in the face as well as in other parts of the body together with autonomic symptoms, with the diagnosis of SFN confirmed by demonstration of reduced IENFD on skin biopsy, and abnormal QST. The second patient gave a history of distal extremity pain and redness, triggered by warmth and relieved by cooling. While these symptoms are commonly reported in IEM, ${ }^{72}$ she also reported autonomic symptoms including increased perspiration, gastrointestinal complaints and hot flashes, which are not characteristic of IEM. The third patient initially experienced discomfort and vasomotor instability over the occiput, which progressed to involve the distal extremities, together with abnormal perspiration, intermittent difficulties with micturition; skin biopsy and QST in this patient were both abnormal, confirming the diagnosis of SFN.

Because facial pain was a prominent part of the clinical picture in one of the patients described in this paper, we assessed the effect of the 1228M mutation 
on excitability of trigeminal ganglion neurons. Our current- clamp analysis demonstrated that the $1228 \mathrm{M}$ variant depolarizes resting membrane potential, reduces current threshold and enhances repetitive firing in these cells. The effect of only one other $\mathrm{Na}_{\mathrm{v}} \mathrm{T} .7$ mutation has been assessed in trigeminal ganglion neurons. We previously reported that the $\mathrm{A}_{1} 632 \mathrm{E} \mathrm{Na}_{\mathrm{v}} 1.7$ mutation, from a patient who displayed a mixed clinical phenotype with features of both IEM and PEPD, produces hyperexcitability in trigeminal ganglion neurons. ${ }^{22}$ The A $632 \mathrm{E}$ mutation, however, produced hyperexcitability in these cells over the entire range of stimulus intensities, while $228 \mathrm{M}$ produces hyperexcitability only at low stimulus intensities. Whether other gain-of-function mutations of $\mathrm{Na}_{\mathrm{v}}{ }^{1} .7$ have similar effects on trigeminal ganglion neurons remains to be determined. The $1228 \mathrm{M}$ substitution is located within the fourth transmembrane segment (S4) within domain I of the $\mathrm{Na}_{\mathrm{v}} \mathrm{l} .7$ channel. The $\mathrm{S}_{4}$ in each of the domains of sodium channels is an amphiphatic helix which is characterized by a repeat motif of positively charged amino acids at every third position. ${ }^{19}$ Non-chargeconserved mutations, S211 P and F216S, in DI/S4 have been linked to IEM, and have been shown to shift voltage-dependence of activation in a hyperpolarizing direction, making it easier to open the mutant channels. ${ }^{23}, 24$ The $1228 \mathrm{M}$ substitution does not change the number of charges in the $\mathrm{S}_{4}$ segment, and reasonably conserves the hydrophobic nature of the side-chain of this residue, and thus might not have been predicted to have a functional effect. A link to function, however, is suggested by the conservation of the 1228 residue at the equivalent position in all voltage-gated sodium channels sequenced to date (Figure 1); 1228 is substituted by the other branched side-chain residue, valine, in $\mathrm{Na}_{\mathrm{v}}$ 1.9. The functional effect of $1228 \mathrm{M}$ might be related to the proximity of the 1228 residue to the cytoplasmic end of the $S_{4}$ segment, which could alter the local structure of the helix in a subtle manner affecting slow-inactivation but not activation. Notably, while the $1228 \mathrm{M}$ variant produced hyperexcitability in both DRG and trigeminal ganglion neurons, only two of the three patients described here reported cranial pain, and it was experienced in the jaw and eyes in one, while it was focused on the scalp in the other.

Our results demonstrate phenotypic diversity in the pain syndromes associated with the $1228 \mathrm{M}$ substitution in the $\mathrm{Na}_{\mathrm{v}} 1.7$ channel in three different patients. Two of these patients were from the same family, which also includes patient 1's two asymptomatic sons who carry the $1228 \mathrm{M} \mathrm{Na}_{\mathrm{v}} 1.7$ variant. Both of these asymptomatic carriers are younger than the age of onset of the three patients presented, and whether they will develop pain in the future is unclear. We have previously noted different ages of onset and different degrees of pain, and an asymptomatic carrier in members of a single family, all housing the G616R $\mathrm{Na}_{\mathrm{v}} \mathrm{T} .7$ mutation. ${ }^{25}$ Whether this phenotypic variability is due to modifier genes, epigenetic factors, and/or environmental factors is not yet clear. The minor 
allele of the $\mathrm{Na}_{\mathrm{v}} 1.7 \mathrm{R} 1150 \mathrm{~W}$ variant, which is known to produce hyperexcitability in DRG neurons, ${ }^{26}$ has been associated with increased pain scores in a number of acquired pain syndromes (osteoarthritis, compressive radiculopathies, traumatic limb amputation), suggesting that environmental factors may, at least in some individuals, act as triggers or increase risk of developing pain. ${ }^{27}$ Most peripheral neuropathies present in a "stocking glove" distribution with sensory abnormalities and pain first appearing in the most distal parts of the limbs (feet, then hands). It has traditionally been held that longer nerve fibers, or the cells giving rise to them, are affected before shorter fibers or the cells giving rise to them. A number of potential mechanisms have been invoked for this length-dependent mode of progression of neuropathy, including impairment of axoplasmic transport, ${ }^{28}$ increased probability of demyelination along longer nerve fibers, ${ }^{29}$ or a higher probability of impairment of calcium homeostasis along longer nerve fibers. ${ }^{30,31}$ However, the present results show that the $\mathrm{Na}_{\mathrm{v}}{ }^{1} \cdot 7$ I228M variant, which impairs slow-inactivation, produces physiological changes in primary afferent neurons (trigeminal ganglion neurons) that innervate the relatively proximal sensory field of the face and scalp, as well as DRG neurons. While we do not know whether there was degeneration of small fibers innervating the face or scalp in these patients, both exhibited degeneration of the relatively long axons, as demonstrated by reduced IENFD on skin biopsy from the leg. In summary, our results demonstrate phenotypic diversity in pain syndromes associated with the $1228 \mathrm{M}$ gain-of-function variant of $\mathrm{Na}_{\mathrm{v}} 1.7$. Importantly, variability in clinical presentation was present not only when comparing patients from different families, but also for patients within a single family. Our findings also demonstrate that the $1228 \mathrm{M}$ variant can increase excitability of trigeminal ganglion as well as DRG neurons. While the mechanism(s) responsible for this phenotypic diversity remain unexplained, our findings suggest that clinical studies, in patients who are carriers of functional variants of sodium channels, should be designed to take phenotypic variability, even within single families, into account.

\section{Materials and methods}

\section{Patients}

The three patients initially studied were part of a cohort of patients aged $\geq$ 18 years with idiopathic SFN, seen at Maastricht University Medical Center Neurological Clinic, with a clinical diagnosis of SFN between 2006 and 2009; this series excluded patients in whom, after a careful work-up, a cause for SFN was identified. A sister of patient 1 was also studied. This study was approved by medical ethics committees at Yale University and Maastricht University Medical 
Center. All aspects of the study were explained and a written informed consent obtained prior to study.

All three patients met strict eligibility criteria for a study on SFN as described by Faber et al. ${ }^{17}$ Subjects were excluded from the study if there was a history or detection after screening of illnesses known to cause SFN, including impaired glucose tolerance, diabetes mellitus, hyperlipidemia, liver/kidney/ thyroid dysfunction, monoclonal-gammopathy, connective tissue disorders, amyloidosis, sarcoidosis, Fabry's disease (alpha-galactosidase, in females combined with GLA-gene sequencing), celiac disease, HIV, alcohol abuse, hemochromatosis, B6 intoxication, anti-phospholipid syndrome neurotoxic drugs (e.g., chemotherapy). ${ }^{17}$

\section{Clinical characterization}

Skin biopsy

Punch biopsy ( $10 \mathrm{~cm}$ above lateral malleolus) specimens were fixed (2\% paraformaldehyde-lysine-sodium periodate at $4^{\circ} \mathrm{C}$ ), cryprotected and stored at $-80^{\circ} \mathrm{C}$ in $20 \%$ glycerol before sectioning $(50 \mu \mathrm{m}) .^{13}$ The numbers of individual nerve fibers crossing the dermal-epidermal junctions were analyzed by brightfield microscopy (Olympus BX 50 stereology workstation, PlanApo oilobjective $40 \times / N A=1.0$ ) in each of three sections, immunostained with polyclonal rabbit antiprotein-geneproduct-9.5 antibody (PGP9.5; Ultraclone, Wellow, IsleofWight, UK). Linear quantification of intraepidermal nerve fiber density (IENF/ $\mathrm{mm}$ ) was compared with age and gender-adjusted normative values. ${ }^{18,32}$

\section{Quantitative sensory testing (QST)}

QST, performed in accordance with previous guidelines, ${ }^{33}$ using a TSA-2001 (Medoc, Ramat-Yishai, Israel) instrument, assessed thresholds at the dorsum of both feet and thenar eminences, using ascending/descending (warm/cool) thermal ramp stimuli delivered through a thermode. ${ }^{34}$ Heat pain modality was also examined. Results were compared with reported normative values. ${ }^{35}$ Measurements were considered abnormal when Z-values exceeded 2.5. A sensory modality was classified as abnormal if results of both method-of-limits and method-of-levels were abnormal. ${ }^{36}$

\section{SCNgA sequence analysis}

\section{Exon screening}

Genomic DNA was extracted from $300 \mu \mathrm{L}$ whole blood using Puregene genomic DNA isolation kit (Gentra-Systems, Minneapolis). All SCN9A coding exons and flanking intronic sequences, and exons encoding 5 ' and 3- untranslated sequences within the complementary DNA, were amplified and sequenced as described previously. ${ }^{37}$ Genomic sequences were compared with reference 
$\mathrm{Na}_{\mathrm{v}} 1.7$ cDNA (NM_002977.3) to identify sequence variations ${ }^{38}$ using Alamut Mutation-Interpretation Software (Interactive-Biosoftware; Rouen, France). A control panel of DNA from 100 healthy Dutch (Caucasian) individuals (200 chromosomes) was also screened.

\section{Plasmids}

The human $\mathrm{Na}_{\mathrm{v}}$ 1.7-AL insert (carrying the adult exon 5, $\mathrm{E}_{5} \mathrm{~A}$, and Long loop 1 ), converted to become TTX-R ( $\mathrm{hNa} \mathrm{V}_{\mathrm{V}} \cdot 7_{\mathrm{R}} / \mathrm{AL}$; designated WT hereinafter) by $\mathrm{Y}_{3} 62 \mathrm{~S}$ substitution, ${ }^{39}$ has been previously described [39]. The I228M mutation was introduced into WT using QuickChange XL II site-directed mutagenesis according to manufacturer recommendations (Stratagene). The full-length inserts of the different clones were sequenced at the Howard Hughes Medical Institute/ Keck Biotechnology Center at Yale University. Sequence analysis used BLAST (National Library of Medicine) and Lasergene (DNAStar, Madison, WI), and confirmed the inserts to be devoid of un-intended mutations.

\section{Transient transfection of HEK293 cells}

Transient transfections of the $h \mathrm{ha}_{\mathrm{v}} \mathrm{\gamma} .7$ together with $h \beta_{1}$ and $h \beta_{2}$ constructs into HEK293 cells were performed using Optifect (Invitrogen) following the recommended protocol by manufacturer. Recordings were performed 20-30 hours after transfection.

\section{Primary sensory neuron isolation and transfection}

Dorsal root ganglia (DRG) and trigeminal ganglia from adult Sprague Dawley rat pups (Po- $\mathrm{P}_{5}$ ) were isolated and then cultured using the same protocol for both. Dissected ganglia were placed in ice cold oxygenated complete saline solution (CSS), which contained (in $\mathrm{mM}$ ) $137 \mathrm{NaCl}, 5.3 \mathrm{KCl}, 1 \mathrm{MgCl}_{2}, 25$ sorbitol, $3 \mathrm{CaCl}_{2}, 10 \mathrm{~N}$-2-hydroxyethylpiperazine- $\mathrm{N}^{\prime}$-2-ethanesulfonic acid (HEPES); $\mathrm{pH}$ 7.2. They were then transferred to an oxygenated, $37^{\circ} \mathrm{C} \mathrm{CSS}$ solution containing $1.5 \mathrm{mg} / \mathrm{ml}$ Collagenase A (Roche Applied Science, Indianapolis, IN) and 0.6 $\mathrm{mM}$ EDTA and incubated with gentle agitation at $37^{\circ} \mathrm{C}$ for $20 \mathrm{~min}$. This solution was then exchanged with an oxygenated, $37^{\circ} \mathrm{C} \mathrm{CSS}$ solution containing $1.5 \mathrm{mg} /$ $\mathrm{ml}$ Collagenase D (Roche Applied Science, Indianapolis, IN), $0.6 \mathrm{mM}$ EDTA and $30 \mathrm{U} / \mathrm{ml}$ papain (Worthington Biochemical, Lakewood, $\mathrm{NJ}$ ) and incubated with gentle agitation at $37^{\circ} \mathrm{C}$ for $20 \mathrm{~min}$. The solution was then aspirated and the ganglia triturated in DRG media (DMEM/Fl2 (1:1) with $100 \mathrm{U} / \mathrm{ml}$ penicillin, $0.1 \mathrm{mg} / \mathrm{ml}$ streptomycin (Invitrogen, Carlsbad, CA) and 10\% fetal calf serum (Hyclone, Logan, UT), which contained $1.5 \mathrm{mg} / \mathrm{ml}$ bovine serum albumin (Sigma-Aldrich, St. Louis, MO) and $1.5 \mathrm{mg} / \mathrm{ml}$ trypsin inhibitor (Roche Applied Science, Indianapolis, IN).

Either WT or $1228 \mathrm{M}$ variant channels were transiently transfected into the DRG or 
trigeminal ganglion neurons, along with enhanced-GFP, by electroporation with a Nucleofector II (Amaxa, Gaithersburg, MD) using Rat Neuron Nucleofector Solution and program G-013, as described previously [39]. The ratio of sodium channel to GFP constructs was 10:1. The transfected neurons were allowed to recover for 5 minutes at $37^{\circ} \mathrm{C}$ in $0.5 \mathrm{ml}$ of $\mathrm{Ca}^{2+}$-free DMEM containing $10 \%$ fetal calf serum. The cell suspension was then diluted with DRG media containing 1.5 $\mathrm{mg} / \mathrm{ml}$ bovine serum albumin and $1.5 \mathrm{mg} / \mathrm{ml}$ trypsin inhibitor, $80 \mu \mathrm{l}$ was plated on $12 \mathrm{~mm}$ circular poly-D-lysine/laminin precoated coverslips (BD Biosciences, Bedford, MA) and the cells incubated at $37^{\circ} \mathrm{C}$ in $5 \% \mathrm{CO}_{2}$ for $30 \mathrm{~min}$. DRC media ( $1 \mathrm{ml} /$ well), supplemented with $50 \mathrm{ng} / \mathrm{ml}$ each of $\mathrm{mNGF}$ (Alomone Labs, Jerusalem, Israel) and GDNF (Peprotec, Rocky Hill, NJ), was then added and the cells maintained at $37^{\circ} \mathrm{C}$ in a $5 \% \mathrm{CO}_{2}$ incubator.

\section{Electrophysiology}

Whole-cell voltage-clamp recordings in HEK293 cells were carried out at 20 $\pm 1^{\circ} \mathrm{C}$ using a peltier temperature controller of the recording chamber. The extracellular solution contained (in $\mathrm{mM}$ ): $140 \mathrm{NaCl}, 3 \mathrm{KCl}, 1 \mathrm{MgCl}_{2}, 1 \mathrm{CaCl}_{2}$, and $10 \mathrm{HEPES}, \mathrm{pH} 7.3$ with $\mathrm{NaOH}$ (adjusted to $320 \mathrm{mOsm}$ with dextrose). The pipette solution contained (in $\mathrm{mM}$ ): $140 \mathrm{CsF}, 10 \mathrm{NaCl}, 2 \mathrm{MgCl}_{2}, 1$ EGTA, $10 \mathrm{HEPES}, \mathrm{pH} 7.3$ with $\mathrm{CsOH}$ (adjusted to $310 \mathrm{mOsm}$ with dextrose). Patchpipettes had a resistance of 1-3 M $\Omega$ when filled with pipette solution. The calculated junction potential (JPcalc included in PCLAMP software) of $9 \mathrm{mV}$ was not compensated. Upon achieving the whole-cell recording configuration, the pipette and cell capacitance were manually minimized using the Axopatch $200 \mathrm{~B}$ (Molecular Devices, Union City, CA) compensation circuitry. To reduce voltage errors, $80-90 \%$ series resistance and prediction compensation was applied. Cells were excluded from analysis if the predicted voltage error exceeded $3 \mathrm{mV}$. The recorded currents were digitized at a rate of $50 \mathrm{kHz}$ after passing through a low-pass Bessel filter setting of $10 \mathrm{kHz}$. The Axopatch $200 \mathrm{~B}$ data were digitized using pCLAMP software (version 10) and a Digidata 1440A interface (Molecular Devices). Linear leak and residual capacitance artifacts were subtracted out using the $\mathrm{P} / \mathrm{N}$ method. The $\mathrm{Na}^{+}$current recordings were initiated after a 5 minute quilibration period once whole-cell configuration was achieved.

Data analysis was performed using Clampfit (Molecular Devices) and Origin (Microcal Software, Northhampton, MA). To generate activation curves, cells were held at $-120 \mathrm{mV}$ and stepped to potentials of -80 to $40 \mathrm{mV}$ for $100 \mathrm{msec}$. Peak inward currents obtained from activation protocols were converted to conductance values using the equation, $G=1 /\left(V_{m}-E_{N a}\right)$, for which $G$ is the conductance, $\mathrm{I}$ is the peak inward current, $\mathrm{V}_{\mathrm{m}}$ is the membrane potential step used to elicit the response and $\mathrm{E}_{\mathrm{Na}}$ is the reversal potential for sodium (determined for each cell using the $\mathrm{x}$-axis intercept of a linear fit of the peak inward current 
responses). Conductance data were normalized by the maximum conductance value and fit with a Boltzmann equation of the form $G=G_{\min }+\left(G_{\max }-G_{\min }\right) /$ $\left(1+\exp \left[\left(V_{1 / 2}-V_{m}\right) / k\right)\right]$, where $V_{1 / 2}$ is the midpoint of activation and $k$ is a slope factor. The kinetics of inactivation were assessed by fitting the falling phase of the currents with a single exponential function. To generate steady-state fastinactivation curves, cells were stepped to inactivating potentials of -140 to 10 $\mathrm{mV}$ for $500 \mathrm{msec}$ followed by a $20 \mathrm{msec}$ step to $-10 \mathrm{mV}$. The protocol for slowinactivation consisted of a 30 second step to potentials varying from -120 to $10 \mathrm{mV}$, followed by a $100 \mathrm{msec}$ step to $-120 \mathrm{mV}$ to remove fast inactivation and a $20 \mathrm{msec}$ step to $-10 \mathrm{mV}$ to elicit a test response. Peak inward currents obtained from steady-state fast-inactivation and slow-inactivation protocols were normalized by the maximum current amplitude and fit with a Boltzmann equation of the form $I=I_{\min }+\left(I_{\max }{ }^{-} I_{\min }\right) /\left(1+\exp \left[\left(V_{m}-V_{1 / 2}\right) / k\right)\right]$, where $V m$ represents the inactivating pre-pulse membrane potential and $V_{1 / 2}$ represents the midpoint of inactivation. For deactivation the cells were held at $-120 \mathrm{mV}$ and tail currents were generated by a brief $0.5 \mathrm{~ms}$ depolarization to $-20 \mathrm{mV}$ followed by a series of repolarizations ranging from -120 to $-40 \mathrm{mV}$. The closing rate of the channels was obtained by fitting the tail currents with a single exponential function.

Whole-cell current-clamp recordings from isolated DRC or trigeminal ganglion neurons were performed using the Axopatch $200 \mathrm{~B}$ amplifier, digitized using the Digidata $1440 \mathrm{~A}$ interface and controlled using PCLAMP software. The bath solution for current-clamp recordings contained (in $\mathrm{mM}$ ): $140 \mathrm{NaCl}, 3 \mathrm{KCl}, 2$ $\mathrm{MgCl}_{2}, 2 \mathrm{CaCl}_{2}$, and $10 \mathrm{HEPES}, \mathrm{pH} 7.3$ with $\mathrm{NaOH}$ (adjusted to $315 \mathrm{mOsm}$ with dextrose). The pipette solution contained (in $\mathrm{mM}$ ): $140 \mathrm{KCl}, 0.5 \mathrm{EGTA}, 5 \mathrm{HEPES}$, and $3 \mathrm{Mg}$-ATP, pH 7.3 with $\mathrm{KOH}$ (adjusted to $300 \mathrm{mOsm}$ with dextrose). The junction potential between these two solutions given by JPcalc was $5 \mathrm{mV}$ but no correction was applied for current-clamp experiments. Recordings were performed on transfected presumptive nociceptive neurons based on the morphology of small diameter $(2 \mathrm{O}-28 \mu \mathrm{m})$ round cell bodies that also exhibited GFP fluorescence. All recordings were performed between $40 \mathrm{hr}$ and $50 \mathrm{hr}$ post-transfection $20 \pm 1^{\circ} \mathrm{C}$. Coverslips were transferred to a perfusable chamber (Warner Instruments, Hamden, CT) and all recordings were initiated within an hour. Whole-cell configuration was obtained in voltage-clamp mode before proceeding to the current-clamp recording mode. Cells with stable $(<$ $10 \%$ variation) resting membrane potentials (RMPs) more negative than -35 $\mathrm{mV}$ and overshooting action potentials ( $>85 \mathrm{mV}$ RMP to peak) were used for further data collection. Input resistance was determined by the slope of a line fit to hyperpolarizing responses to current steps of 10-35 pA. Threshold was determined by the first action potential elicited by a series of depolarizing current injections that increased in $5 \mathrm{pA}$ increments. The number of action potentials 
elicited in response to depolarizing current injections of $500 \mathrm{msec}$ duration was also measured. After-hypolarization currents, and amplitude and width of action potentials were not formally analyzed in this study. Data are expressed as means \pm standard error (SEM). Statistical significance was determined by Student's t-test, Mann-Whitney test (firing frequency) or z-test (frequency of spontaneous firing). 


\section{References}

1. Toledo-Aral, J.J. et al. Identification of $\mathrm{PN}_{1}$, a predominant voltage-dependent sodium channel expressed principally in peripheral neurons. Proc Natl Acad Sci U S A 94, 1527-32 (1997).

2. Rush, A.M. et al. A single sodium channel mutation produces hyper- or hypoexcitability in different types of neurons. Proc Natl Acad Sci U S A 103, 8245-50 (2006).

3. Sangameswaran, L. et al. A novel tetrodotoxin-sensitive, voltage-gated sodium channel expressed in rat and human dorsal root ganglia.J Biol Chem 272, $14805-9$ (1997).

4. Persson, A.K. et al. Sodium-calcium exchanger and multiple sodium channel isoforms in intra-epidermal nerve terminals. Mol Pain 6, 84 (2010).

5. Cummins, T.R., Howe, J.R. \& Waxman, S.G. Slow closed-state inactivation: a novel mechanism underlying ramp currents in cells expressing the hNE/PN1 sodium channel. J Neurosci 18, 9607-19 (1998).

6. Waxman, S.G. Neurobiology: a channel sets the gain on pain. Nature 444, 831-2 (2006).

7. Dib-Hajj, S.D., Cummins, T.R., Black, J.A. \& Waxman, S.G. Sodium channels in normal and pathological pain. Annu Rev Neurosci 33, 325-47 (2010).

8. Fertleman, C.R. et al. Paroxysmal extreme pain disorder (previously familial rectal pain syndrome). Neurology 69, 586-95 (2007)

9. Fertleman, C.R. et al. SCN9A mutations in paroxysmal extreme pain disorder: allelic variants underlie distinct channel defects and phenotypes. Neuron 52, 767-74 (2006).

10. Lacomis, D. Small-fiber neuropathy. Muscle Nerve 26, 173-88 (2002).

11. Lauria, G. Small fibre neuropathies. Curr Opin Neurol 18, 591-7 (2005).

12. Stewart, J.D., Low, P.A. \& Fealey, R.D. Distal small fiber neuropathy: results of tests of sweating and autonomic cardiovascular reflexes. Muscle Nerve 15, 661-5 (1992).

13. Lauria, G. et al. European Federation of Neurological Societies/Peripheral Nerve Society Guideline on the use of skin biopsy in the diagnosis of small fiber neuropathy. Report of a joint task force of the European Federation of Neurological Societies and the Peripheral Nerve Society. Eur J Neurol 17, 903-12, e44-9 (2010)

14. Tesfaye, S. et al. Diabetic neuropathies: update on definitions, diagnostic criteria, estimation of severity, and treatments. Diabetes Care 33, 2285-93 (2010).

15. Devigili, G. et al. The diagnostic criteria for small fibre neuropathy: from symptoms to neuropathology. Brain 131, 1912-25 (2008).

16. Bednarik, J. et al. Etiology of small-fiber neuropathy. J Peripher Nerv Syst 14, 177-83 (2009).

17. Faber, C.G. et al. Gain of function $\mathrm{Na}(\mathrm{V}) 1.7$ mutations in idiopathic small fiber neuropathy. Ann Neurol 7 , 26-39 (2012).

18. Lauria, G. et al. Intraepidermal nerve fiber density at the distal leg: a worldwide normative reference study. J Peripher Nerv Syst 15, 202-7 (2010).

19. Catterall, W.A., Goldin, A.L. \& Waxman, S.G. International Union of Pharmacology. XLVII. Nomenclature and structure-function relationships of voltage-gated sodium channels. Pharmacol Rev 57, 397-409 (2005).

20. Singh, N.A. et al. A role of $\mathrm{SCN}_{9} \mathrm{~A}$ in human epilepsies, as a cause of febrile seizures and as a potential modifier of Dravet syndrome. PLoS Genet 5, elooo649 (2009).

21. Drenth, J.P. \& Waxman, S.G. Mutations in sodium-channel gene SCNgA cause a spectrum of human genetic pain disorders. J Clin Invest 117, 3603-9 (2007).

22. Estacion, M. et al. NaV1.7 gain-of-function mutations as a continuum: A $632 \mathrm{E}$ displays physiological changes associated with erythromelalgia and paroxysmal extreme pain disorder mutations and produces symptoms of both disorders. J Neurosci 28, 11079-88 (2008).

23. Estacion, M. et al. Can robots patch-clamp as well as humans? Characterization of a novel sodium channel mutation. J Physiol 588, 1915-27 (2010).

24. Choi, J.S., Dib-Hajj, S.D. \& Waxman, S.G. Inherited erythermalgia: limb pain from an S4 charge-neutral Na channelopathy. Neurology 67, 1563-7 (2006).

25. Choi, J.S. et al. Alternative splicing may contribute to time-dependent manifestation of inherited erythromelalgia. Brain 133, 1823-35 (2010).

26. Estacion, M. et al. A sodium channel gene $\mathrm{SCNgA}$ polymorphism that increases nociceptor excitability. Ann Neurol 66, 862-6 (2009). 
27. Reimann, F. et al. Pain perception is altered by a nucleotide polymorphism in SCNgA. Proc Natl Acad Sci U S A 107, 5148-53 (2010).

28. Sabin, T.D. Classification of peripheral neuropathy: the long and the short of it. Muscle Nerve 9, 711-9 (1986).

29. Waxman, S.G., Brill, M.H., Geschwind, N., Sabin, T.D. \& Lettvin, J.Y. Probability of conduction deficit as related to fiber length in random-distribution models of peripheral neuropathies. J Neurol Sci 29, 39-53 (1976).

30. Viader, A. et al. Schwann cell mitochondrial metabolism supports long-term axonal survival and peripheral nerve function. J Neurosci 31, 10128-40 (2011).

31. Baloh, R.H. Mitochondrial dynamics and peripheral neuropathy. Neuroscientist 14, $12-8$ (2008).

32. Bakkers, M. et al. Intraepidermal nerve fiber density and its application in sarcoidosis. Neurology 73, 11428 (2009).

33. Shy, M.E. et al. Quantitative sensory testing: report of the Therapeutics and Technology Assessment Subcommittee of the American Academy of Neurology. Neurology 60, 898-904 (2003).

34. Reulen, J.P., Lansbergen, M.D., Verstraete, E. \& Spaans, F. Comparison of thermal threshold tests to assess small nerve fiber function: limits vs. levels. Clin Neurophysiol 114, 556-63 (2003).

35. Yarnitsky, D. \& Sprecher, E. Thermal testing: normative data and repeatability for various test algorithms. J Neurol Sci 125, 39-45 (1994).

36. Hoitsma, E. et al. Abnormal warm and cold sensation thresholds suggestive of small-fibre neuropathy in sarcoidosis. Clin Neurophysiol 114, 2326-33 (2003).

37. Drenth, J.P. et al. SCNgA mutations define primary erythermalgia as a neuropathic disorder of voltage gated sodium channels. J Invest Dermatol 124, 1333-8 (2005).

38. Klugbauer, N., Lacinova, L., Flockerzi, V. \& Hofmann, F. Structure and functional expression of a new member of the tetrodotoxin-sensitive voltage-activated sodium channel family from human neuroendocrine cells. EMBOJ 14, 1084-90 (1995).

39. Dib-Hajj, S.D. et al. Transfection of rat or mouse neurons by biolistics or electroporation. Nat Protoc 4 $1118-26$ (2009) 



\title{
Chapter 5
}

\section{$\mathrm{Na}_{\mathrm{v}}$ 1.7-related small fiber neuropathy: impaired slow-inactivation and DRG neuron hyperexcitability}

\author{
C. Han ${ }^{a, b *}$, J.G.J. Hoeijmakers $*$, H.S. Ahn ${ }^{a, b}$, P. Zhao, P. Shah ${ }^{a, b}$, G. Lauriad, \\ M.M. Gerritse, R.H.M. te Morschef, S.D. Dib-Haja, , J.P.H. Drenthf, C.G. Faber, \\ MD, I.S.J. Merkies',g, S.G. Waxman ${ }^{a, b}$. \\ * co-first authorship
}

${ }^{a}$ Department of Neurology, Yale University School of Medicine, New Haven, CT, USA ${ }^{b}$ Center for Neuroscience and Regeneration Research, Veterans Affairs Medical Center,

West Haven, CT, USA

'Department of Neurology, Maastricht University Medical Center, Maastricht ${ }^{d}$ Neuromuscular Diseases Unit, IRCCS Foundation, Carlo Besta Neurological Institute, Milan, Italy

${ }^{\text {eDepartment }}$ of Clinical Genomics, Maastricht University Medical Center, Maastricht fDepartment of Gastroenterology and Hepatology, Radboud University Nijmegen Medical

Center, Nijmegen ${ }^{\text {g} D e p a r t m e n t ~ o f ~ N e u r o l o g y, ~ S p a a r n e ~ H o s p i t a l, ~ H o o f d d o r p ~}$

Neurology. 2012;78(21):1635-43; Published with permission from Wolters Kluwer Health 


\section{Abstract}

Objectives: Although small fiber neuropathy (SFN) often occurs without apparent cause, the molecular etiology of idiopathic SFN (I-SFN) has remained enigmatic. Sodium channel $\mathrm{Na}_{\mathrm{v}} 1.7$ is preferentially expressed within dorsal root ganglion (DRG) and sympathetic ganglion neurons and their small-diameter peripheral axons. We recently reported the presence of $\mathrm{Na}_{\mathrm{v}} \mathrm{T} .7$ variants that produce gainof-function changes in channel properties in $28 \%$ of patients with painful I-SFN and demonstrated impaired slow-inactivation in one of these mutations after expression within HEK293 cells. Here we show that the $1739 \mathrm{~V} \mathrm{Na} \mathrm{v}_{\mathrm{v}}$.7 variant in a patient with biopsy-confirmed I-SFN impairs slow-inactivation within DRG neurons and increases their excitability.

Methods: A patient with SFN symptoms including pain, and no identifiable underlying cause, was evaluated by skin biopsy, quantitative sensory testing, nerve conduction studies, screening of genomic DNA for variants in $\mathrm{SCN}_{9} A$, and functional analysis.

Results: Voltage-clamp analysis following expression within DRG neurons revealed that the $\mathrm{Na}_{\mathrm{v}} 1.7 / 1739 \mathrm{~V}$ substitution impairs slow-inactivation, depolarizing the midpoint $\left(\mathrm{V}_{1 / 2}\right)$ by $5.6 \mathrm{mV}$, and increasing the noninactivating component at $10 \mathrm{mV}$ from $16.5 \%$ to $22.2 \%$. Expression of $1739 \mathrm{~V}$ channels within DRG neurons rendered these cells hyperexcitable, reducing current threshold and increasing the frequency of firing evoked by graded suprathreshold stimuli. Conclusions: These observations provide support, from a patient with biopsyconfirmed SFN, for the suggestion that functional variants of $\mathrm{Na}_{\mathrm{v}} \mathrm{1} .7$ that impair slow-inactivation can produce DRG neuron hyperexcitability that contributes to pain in SFN. $\mathrm{Na}_{\mathrm{v}} \mathrm{T} .7$ channelopathy-associated SFN should be considered in the differential diagnosis of cases of SFN in which no other cause is found. 
Small fiber neuropathy (SFN) is a disorder of thinly myelinated and unmyelinated nerve fibers typically dominated by neuropathic pain and autonomic symptoms. ${ }^{1.6}$ Sodium channel $\mathrm{Na}_{\mathrm{y}} \mathrm{T} .7$, encoded by $\mathrm{SCN} 9 \mathrm{~A}$, is abundantly expressed in dorsal root ganglion (DRG) and sympathetic ganglion neurons ${ }^{7,8}$ and their axons ${ }^{9}$ and opens in response to small depolarizations close to resting potential. ${ }^{10}$ Gain-of-function mutations in $\mathrm{Na}_{\mathrm{v}} \mathrm{T} .7$ that enhance activation and impair fastinactivation cause the painful disorders inherited erythromelalgia (IEM) ${ }^{11,12}$ and paroxysmal extreme pain disorder (PEPD). ${ }^{13}$ We recently found $\mathrm{Na}_{\mathrm{v}}{ }^{1.7}$ variants that produce multiple gain-of-function biophysical changes, distinct from those observed with IEM" ${ }^{11}$ and PEPD, ${ }^{13}$ in nearly $30 \%$ of patients with biopsyconfirmed idiopathic SFN (I-SFN), i.e., SFN without apparent cause..$^{14}$ In that report we demonstrated that one of these mutations impairs slow-inactivation when expressed in a heterologous system, HEK293 cells. Here we show that the $\mathrm{Na}_{\mathrm{v}} 1.7 / 1739 \mathrm{~V}$ substitution, from a patient with biopsyconfirmed SFN and severe pain, impairs channel slow-inactivation when expressed within DRG neurons, and increases excitability of these cells. These results suggest that $\mathrm{Na}_{\mathrm{v}} \mathrm{1} .7$ functional variants that impair slow-inactivation can produce DRG neuron hyperexcitability associated with SFN.

\section{Methods}

\section{Patient evaluation}

The patient, evaluated as part of a series reported by Faber et al., ${ }^{14}$ at Maastricht University Medical Center neurologic clinic, met strict eligibility and exclusion criteria for SFN as described by Faber et al. ${ }^{14}$

\section{Standard protocol approvals, registrations, and patient consents}

This study was approved by medical ethics committees at Yale University and Maastricht University Medical Center. All aspects of the study were explained and written informed consent obtained prior to study initiation.

\section{Clinical characterization}

\section{Skin biopsy}

Punch biopsy ( $10 \mathrm{~cm}$ above lateral malleolus) specimens were fixed (2\% paraformaldehyde-lysine-sodium periodate, $4^{\circ} \mathrm{C}$ ), cryoprotected, and stored $\left(-80^{\circ} \mathrm{C}\right)$ in $20 \%$ glycerol before sectioning $(50 \mu \mathrm{m}) . .^{15}$ Numbers of individual nerve fibers crossing dermalepidermal junctions were analyzed in each of 3 sections, immunostained with polyclonal rabbit antiprotein-gene-product-9.5 antibody (PGP9.5; Ultraclone, Wellow, Isle-of-Wight, UK), by bright-field microscopy (Olympus BX50 stereology-workstation, PlanApo oil-objective 40X/ 
$N A=1.0$ ). Linear quantification of intraepidermal nerve fiber density (IENF/ $\mathrm{mm}$ ) was compared with age- and gender-adjusted normative values. ${ }^{16}$

Quantitative sensory testing

Quantitative sensory testing (QST), performed according to previous guidelines ${ }^{17}$ (TSA-2001 instrument; Medoc, Ramat-Yishai, Israel), assessed thresholds at the dorsum of both feet and thenar eminences, using ascending/ descending (warm/cool) thermal ramp stimuli. ${ }^{18}$ Heat pain modality was also examined. Results, compared with normative values, ${ }^{19}$ were considered abnormal for $Z$ values $>2.5$. Sensory modalities were classified as abnormal if results of both method-of-limits and method-of-levels were abnormal. ${ }^{20}$

\section{SCNgA sequence analysis}

Exon screening

Genomic DNA isolation, and amplification/analysis of $S C N 9 A$ coding exons and flanking intronic sequences, and exons encoding 5' and 3-untranslated sequences within complementary DNA, were described previously. ${ }^{21}$ Exon sequences were compared with reference $\mathrm{Na}_{\mathrm{v}} 1.7$ cDNA (NM_002977.3) to identify variation $\mathrm{s}^{22}$ using Alamut Mutation-Interpretation Software (InteractiveBiosoftware; Rouen, France). DNA from 1,000 Caucasian control subjects $(2,000$ chromosomes) from the same geographical region as the index patient was analyzed for C.2215 A $>\mathrm{C}$ substitution by PCR and high-resolution-meltingcurve analysis. Forwardprimer 3'-TTTTATTGTAATCGATCCTTTTCTAG-5' and reverse-primer 3'-ATTCGCTCGTCTTCCATACC-5' and fluorescent dye EvaGreen (Biotium, Hayward, CA) were used to amplify exon 13; presence of variant alleles was determined from melting curves $\left(65^{\circ} \mathrm{C}\right.$ to $95^{\circ} \mathrm{C}$; ramp rate $0.1^{\circ} \mathrm{C} / 10$ seconds; CFX96TM Real-Time-PCR-Detection System; BioRad, Hercules, CA), analyzed using Precision-Melt software (BioRad).

\section{Functional analysis}

$D R G$ neuron transfection; voltage-clamp recordings

As in previous studies on several $\mathrm{Na}_{\mathrm{v}} 1.7$ mutations, ${ }^{23}, 24$ initial voltage-clamp assessment did not reveal biophysical changes in properties of $1739 \mathrm{~V}$ channels expressed within HEK293 cells. Voltage-clamp analysis was therefore carried out after biolistic (Helios Gene-Gun, Bio-Rad) transfection of DRG neurons ${ }^{23-25}$ cultured from 4- to 8-week-old $\mathrm{Na}_{\mathrm{v}}$ 1.8-null mice. ${ }^{26}$ Wild-type or $1739 \mathrm{~V}$ plasmids were mixed with green fluorescent protein (GFP) DNA (channel:GFP ratio 3:1) using $1.0 \mu \mathrm{m}$ gold particles. Absence of $\mathrm{Na}_{\mathrm{v}} \mathrm{1} .8 \mathrm{TTX}-\mathrm{R}$ current in these DRG neurons facilitated analysis of WT and $1739 \mathrm{~V} \mathrm{Na} 1.7$ channels, which were rendered TTX-R. ${ }^{27}$ Protocols for care and sacrifice of mice were approved by the 
Veterans Administration Connecticut IACUC.

Whole-cell patch-clamp recordings were obtained in voltage-clamp mode at room temperature $\left(\sim 21^{\circ} \mathrm{C}\right), 40-48$ hours after transfection using $0.8-1.5 \mathrm{M} \Omega$ electrodes and EPC-9 amplifier and Pulse 8.5 (HEKA, Germany). Cells transfected with wildtype or $1739 \mathrm{~V}$ channels were selected using identical criteria, which required that cells be GFP-fluorescent, isolated, round with diameter $20-30 \mu \mathrm{m}$ and 1 or fewer neurites (to reduce space-clamp artifact), smooth membranes, peak current $>1 \mathrm{nA}$. Voltage errors were minimized using $80 \%$ 90\% series resistance compensation and linear leak subtraction; capacitance artifact was cancelled using computer-controlled circuitry. Recordings were started 5 minutes after establishing whole-cell configuration. The pipette solution contained (mM): $140 \mathrm{CsF} / 1 \mathrm{EGTA} / 10 \mathrm{NaCl} / 10 \mathrm{HEPES} / \mathrm{pH} 7.3$ with $\mathrm{CsOH}$ (adjusted to $315 \mathrm{mOsm}$ with dextrose). The bathing solution was $(\mathrm{mM}) 140 \mathrm{NaCl} / 3 \mathrm{KCl} / 1$ $\mathrm{MgCl}_{2} / 1 \mathrm{CaCl}_{2} / 20 \mathrm{TEA}-\mathrm{Cl} / 5 \mathrm{CsCl} / 0.1 \mathrm{CdCl}_{2} / 0.0003 \mathrm{TTX} / 10 \mathrm{HEPES}, \mathrm{pH} 7.3$ with $\mathrm{NaOH}$ (adjusted to $320 \mathrm{mOsm}$ with dextrose).

To assess deactivation, cells were held at $-100 \mathrm{mV}$ and tail currents generated by $0.5 \mathrm{msec}$ depolarization to $-20 \mathrm{mV}$ followed by a series of repolarizations (-100 to $-40 \mathrm{mV}$ ). Deactivation kinetics were measured using single exponential fits to estimate current decay time at potentials from -100 to $-40 \mathrm{mV}$. Steady-state fast-inactivation was examined using a series of 500-msec prepulses from -150 to $\circ \mathrm{mV}$ followed by test pulses to $-10 \mathrm{mV}$. Steadystate slow-inactivation was assessed using a $20-\mathrm{msec}$ pulse to $-10 \mathrm{mV}$ after a 30-s prepulse to potentials ranging from -130 to $10 \mathrm{mV}$ followed by a 100-msec pulse to $-120 \mathrm{mV}$ to remove fastinactivation. Midpoint $\left(\mathrm{V}_{1 / 2}\right)$ values were measured from Boltzmann fits calculated for each cell and averaged. The offset (noninactivating component) of current at $10 \mathrm{mV}$, the maximum depolarization potential, was determined from Boltzmann fit of the slow-inactivation curve. Recovery from slow-inactivation was assessed using a 2-pulse protocol and varying the interpulse interval and interpulse potential. The neuron was first given a test pulse to $-10 \mathrm{mV}$ for 20 msec to measure peak current, followed by a 10-s pulse to $-10 \mathrm{mV}$ to induce steady-state slow-inactivation and a variable duration recovery period, then given a second test pulse to $-10 \mathrm{mV}$ again. For each recovery potential, peak current response to the second test pulse was normalized to the response to the first test pulse, plotted as a function of recovery period duration, and fit with a single exponential.

\section{Current-clamp recordings}

Current-clamp analysis in transfected DRG neurons was carried out using previously described methods. ${ }^{23,24,28}$ Briefly, DRG from 4- to 8-week-old SpragueDawley rats were harvested and dissociated. ${ }^{24}$ Wild-type $\mathrm{Na}_{\mathrm{v}} 1.7_{\mathrm{R}}$ or $1739 \mathrm{~V}$ channels and GFP constructs (channel:GFP ratio 5:1) were electroporated into 
DRG neurons using Rat- Neuron-Nucleofector Solution (Amaxa, Gaithersburg, MD) as described previously. ${ }^{28}$ Current-clamp recordings were obtained $40-48$ hours after transfection from cells transfected with wildtype or $1739 \mathrm{~V}$ channels using identical criteria, which required that cells be GFP fluorescent, isolated, round with diameter 20 to $30 \mu \mathrm{m}$, smooth membranes, with stable resting potentials more negative than $-35 \mathrm{mV}$ and action potential amplitudes $>80 \mathrm{mV}$. Electrodes (1-3 M 2 ) were filled with pipette solution $(\mathrm{mM}): 140 \mathrm{KCl} / 0.5 \mathrm{EGTA} / 5$ $\mathrm{HEPES} / 3 \mathrm{Mg}-\mathrm{ATP} / \mathrm{pH} 7.3$ with $\mathrm{KOH}$ (adjusted to $315 \mathrm{mOsm}$ with dextrose). The extracellular solution contained $(\mathrm{mM}): 140 \mathrm{NaCl} / 3 \mathrm{KCl} / 2 \mathrm{MgCl}_{2} / 2 \mathrm{CaCl}_{2} / 10$ HEPES, pH 7.3 with $\mathrm{NaOH}$ (adjusted to $320 \mathrm{mOsm}$ with dextrose). Wholecell configuration was obtained in voltage-clamp mode before proceeding to current-clamp. Threshold was determined by the first action potential elicited by a series of depolarizing current injections ( $5 \mathrm{pA}$ increments). Responses to sustained depolarization were assessed using $500 \mathrm{msec}$ depolarizations (25 PA increments).

\section{Data analysis}

Clinical characteristics are descriptively presented. Electrophysiologic data were analyzed using PulseFit 8.74 (HEKA Electronics) and Origin 8.1 (Microcal, Northampton, MA), and presented as means \pm standard error. Statistical significance was determined by unpaired Student $t$ tests or Mann-Whitney test (firing frequency).

\section{Results}

\section{Case history}

At age 14, the patient experienced episodic burning pain, flushing, and itching of the face, lower legs, and feet, induced and aggravated by exercise, warmth, or lying under covers. Cooling relieved the pain. The patient also complained of scalp pain, e.g., while brushing or touching her hair. She gradually developed hyperhydrosis, dry mouth and eyes, blurred vision, orthostatic dizziness, alternating constipation/diarrhea, heart palpitations, and episodic swallowing difficulties. At age 49 she developed polyarticular pain and was diagnosed with fibromyalgia. Treatment with antidepressants (R/duloxetine, amitriptyline) was unsuccessful. Physical examination demonstrated purple skin of the hands and patchy allodynia at both feet and lower legs. There were no signs of large nerve fiber involvement.

Blood tests and chest $\mathrm{x}$-ray were normal. Normal nerve conduction studies excluded large fiber involvement. QST demonstrated abnormal thresholds for warmth sensation in the left hand (levels $34.8^{\circ} \mathrm{C}$, normative value $<33.4^{\circ} \mathrm{C}$; limits 
$36.0^{\circ} \mathrm{C}$, normative value $<35.3^{\circ} \mathrm{C}$ ) and left foot (levels $44.5^{\circ} \mathrm{C}$, normative value $<41.3^{\circ} \mathrm{C}$; limits $46.1^{\circ} \mathrm{C}$, normative value $<45.1^{\circ} \mathrm{C}$ ); thresholds for cold sensation were abnormal in the left foot (levels $21.1^{\circ} \mathrm{C}$, normative value $>25.9^{\circ} \mathrm{C}$; limits $18.2^{\circ} \mathrm{C}$, normative value $\left.>25.4^{\circ} \mathrm{C}\right) . .^{19}$

Intraepidermal nerve fiber density in the lower leg was $3.4 / \mathrm{mm}$, lower than ageand gender-matched normative values (lower limit 4.1/ $/ \mathrm{mm}$; figure 1). ${ }^{16}$

The proband's 2 sons and sister, who did not consent to DNA analysis, and deceased father, manifested similar symptoms.

Figure 1 Histologic confirmation of small fiber neuropathy in this patient
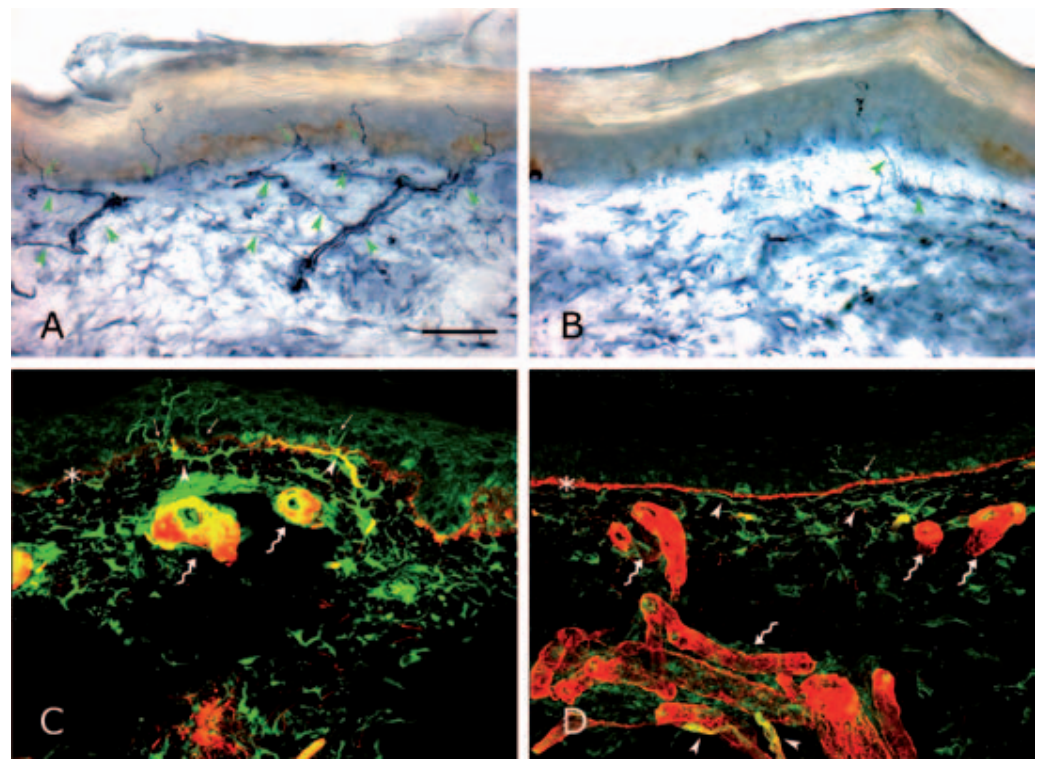

Legend to figure 1. Skin biopsy ( $50 \mu \mathrm{m}$ sections) from a healthy subject $(A, C)$ and the patient (B, D). (A, B) Bright-field images of immunostaining with polyclonal anti-PGP9.5 antibody. (C, D) Confocal images of double-staining with anti-PGP9.5 and anti-collagen IV antibodies to identify the dermal-epidermal junction. Note the dramatic reduction of intraepidermal nerve fibers (arrows) and dermal nerve bundles (arrowheads) in the patient. Curled arrows indicate blood vessels. Bar is $50 \mu \mathrm{m}$.

\section{Genetic analysis}

Sequencing of $S C N_{9} A$ coding exons revealed the c.2215A>C, Na $1.7 / 1739 \mathrm{~V}$ substitution. The 1739 residue, located within the first transmembrane segment in domain II, is conserved in all mammalian $\mathrm{Na}_{\mathrm{v}} 1.7$ orthologues reported to date, and in all human voltage-gated sodium channels (figure 2). Sequencing of DNA from an asymptomatic brother did not reveal any $S C N g A$ variants. The c.2215A>C substitution in exon 13 of SCNgA was found in $14(1.4 \%)$ of 1,000 control subjects (all heterozygous; $0.7 \%$ of 2,000 chromosomes). 
Figure 2. Conservation of 1739 in voltage-gated sodium channels

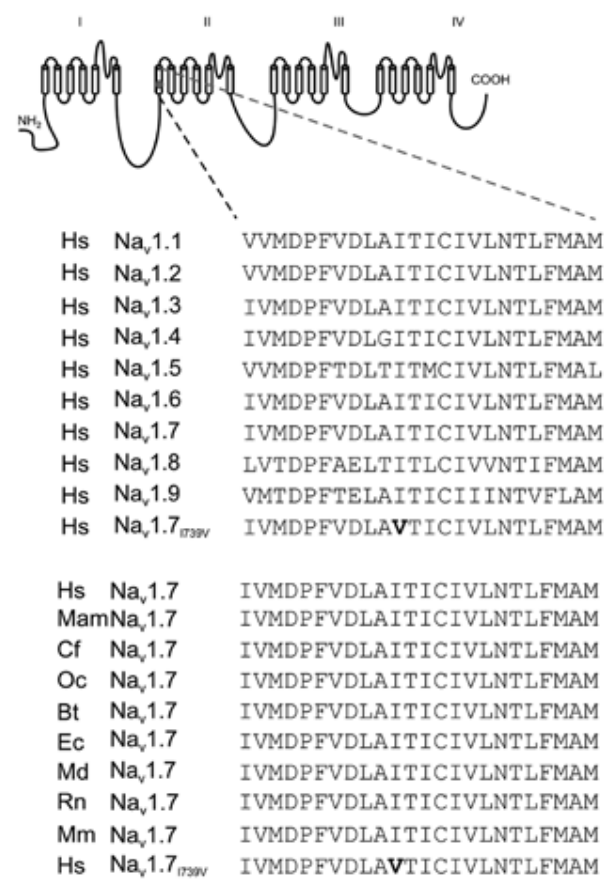

Legend to figure 2. Schematic of a voltage-gated sodium channel showing the location of the $1739 \mathrm{~V}$ substitution and the aligned sequences for the $\mathrm{Na}_{\mathrm{v}}$ 1 DIIS1. I739 is conserved in all known human voltage-gated sodium channels, and the $\mathrm{Na}_{\mathrm{v}} 1.7$ orthologues from mammals.

\section{Functional analysis}

Voltage-clamp analysis.

Voltage-clamp analysis of $1739 \mathrm{~V}$ channels in transfected DRG neurons demonstrated selective impairment of slow-inactivation. Current densities were not significantly different (WT: $806 \pm 71 \mathrm{pA} / \mathrm{pF}, \mathrm{n}=48$; $1739 \mathrm{~V}: 717 \pm 96 \mathrm{pA} / \mathrm{pF}$, $n=41$; representative currents shown in figure $3, A$ and $B$ ). Activation voltage dependence, assessed with depolarizing test pulses from $-80 \mathrm{mV}$ to $+60 \mathrm{mV}$, was not significantly different (activation midpoints determined from fitting with a Boltzmann function: WT: $-23.1 \pm 1.7 \mathrm{mV}, \mathrm{n}=19$; l739V: $-21.6 \pm 1.5 \mathrm{mV}, \mathrm{n}=$ 20) (figure ${ }_{3} \mathrm{C}$ ). Steady-state fast-inactivation midpoint (WT: $-68.7 \pm 1.8 \mathrm{mV}, \mathrm{n}=$ 17; I739V: $-66.0 \pm 1.5 \mathrm{mV}, \mathrm{n}=19$ ) (figure $3 \mathrm{C}$ ) and deactivation kinetics (figure $3 \mathrm{D}$ ) were not significantly different between wild-type and $1739 \mathrm{~V}$ channels.

Slow-inactivation was impaired for $1739 \mathrm{~V}$ channels, with the midpoint depolarized by $5.6 \mathrm{mV}$ (WT: $-61.4 \pm 1.3 \mathrm{mV}, \mathrm{n}=15 ; 1739 \mathrm{~V}:-55.8 \pm 1.8 \mathrm{mV}, \mathrm{n}=16 ; p$ $<0.05$; figure $3 \mathrm{E}) .1739 \mathrm{~V}$ increased the component of noninactivating channels measured at $10 \mathrm{mV}(\mathrm{WT}: 16.5 \pm 1.5 \%, \mathrm{n}=15 ; \mathrm{I} 739 \mathrm{~V}: 22.2 \pm 2.0 \%, \mathrm{n}=16 ; p<0.05)$. 
Recovery from slow-inactivation was investigated at 2 different recovery potentials, $-100 \mathrm{mV}$ and $-70 \mathrm{mV}$. A slightly faster rate of recovery at $-70 \mathrm{mV}$ was observed for $1739 \mathrm{~V}(\tau=3.35 \pm 0.22 \mathrm{~s}, \mathrm{n}=23$ ) compared to wild-type channels $(\tau=4.11 \pm 0.23 \mathrm{~s}, \mathrm{n}=23, p<0.05)$, while the rate of recovery at $-100 \mathrm{mV}$ was similar between wild-type $(\tau=0.94 \pm 0.06 \mathrm{~s}, \mathrm{n}=21)$ and $1739 \mathrm{~V}(\tau=0.91 \pm 0.07$ $s, n=22$ ). Depolarized slow-inactivation and increased offset both are expected to increase the number of channels available for activation at potentials positive to $-100 \mathrm{mV}$, including potentials close to resting potential of DRG neurons.

Figure 3. Impaired slow-inactivation in I739V channels

A

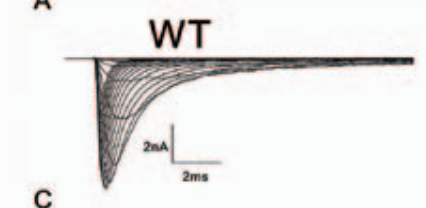

B

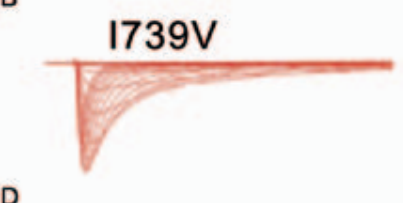

D

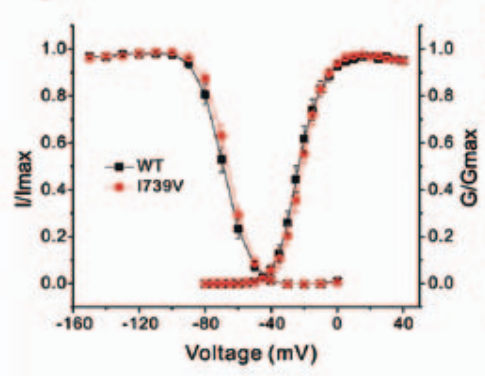

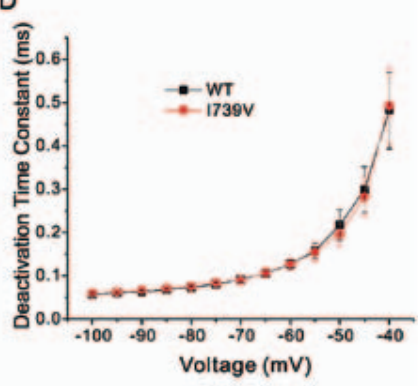

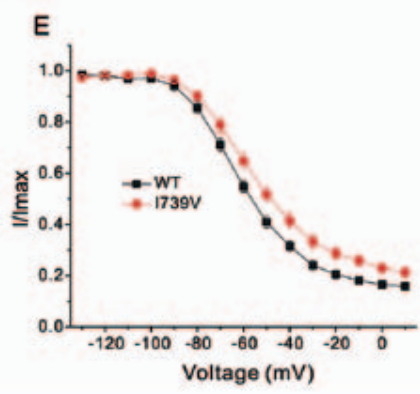

Legend to figure 3. Voltage-clamp analysis of wild-type and I739V channels in DRG neurons. Representative current traces recorded from DRG neurons expressing WT (A) or I739V (B), evoked by voltage steps (100 msec) from $-80 \mathrm{mV}$ to $60 \mathrm{mV}$ in $5-\mathrm{mV}$ increments, from a holding potential of $-100 \mathrm{mV}$. (C) Comparison of activation and steady-state fast-inactivation for wildtype and $1739 \mathrm{~V}$ channels. $1739 \mathrm{~V}$ does not alter activation or fast-inactivation. (D) Comparison of time constants for deactivation. $1739 \mathrm{~V}$ displays similar deactivation rates as wild-type channels. (E) Comparison of steady-state slow-inactivation curves between wild-type and $1739 \mathrm{~V}$ mutant channels. I739V mutation impaired steady-state slow-inactivation by $5.6 \mathrm{mV}$ and increased the noninactivating component at $10 \mathrm{mV}$ by $5.7 \%$. 
While the time constant for recovery from inactivation was reduced at $-70 \mathrm{mV}$, the difference was $<1 \mathrm{msec}$, and the functional significance of this change is not clear.

\section{Current-clamp analysis}

We used current-clamp recording to assess the effect of the $1739 \mathrm{~V}$ channels on DRG neuron excitability. Input resistance was similar in DRG neurons expressing wild-type $(1,037 \pm 107 \mathrm{M} \Omega, \mathrm{n}=29)$ and $1739 \mathrm{~V}$ channels $(1,029 \pm$ $67 \mathrm{M} \Omega, \mathrm{n}=28 ; p>0.05)$. $1739 \mathrm{~V}$ channels produced a significant depolarizing shift in resting potential (WT: $-56.4 \pm 0.8 \mathrm{mV}, \mathrm{n}=29 ; 1739 \mathrm{~V}:-51.4 \pm 1.1 \mathrm{mV}, \mathrm{n}$ $=28 ; p<0.001$ ). Current threshold, i.e., injected current required to produce a single action potential (figure $4, A$ and $B$ ), was reduced significantly in cells expressing I739V (WT: $212 \pm 15$ pA, $\mathrm{n}=29$; I739V: $146 \pm 14 \mathrm{pA}, \mathrm{n}=28 ; p<0.01$ ). DRG neurons expressing $1739 \mathrm{~V}$ channels generated more action potentials in response to sustained depolarizing stimuli compared with neurons expressing wild-type channels. Responses of representative neurons expressing wild-type or $1739 \mathrm{~V}$ channels, to 500 msec current steps at $1 \mathrm{x}, 1.5 \mathrm{x}$, and $2 \mathrm{x}$ current threshold, are shown in figure $4, \mathrm{D}-\mathrm{H}$. Only 2 action potentials were produced by neurons expressing wild-type channels in response to a stimulus at $2 x$ current threshold (figure 4F), while DRG neurons expressing $1739 \mathrm{~V}$ channels generated multiple action potentials (figure $4 \mathrm{l}$ ). Responses of DRG neurons expressing $1739 \mathrm{~V}$ channels were increased over a range of graded suprathreshold stimulation levels (figure 4J)).

\section{Discussion}

Sodium channel $\mathrm{Na}_{\mathrm{v}} 1.7$ is abundantly expressed within DRG and sympathetic ganglion neurons ${ }^{7,8}$ and their axons, including small-diameter peripheral axons. ${ }^{9}$ We recently reported the presence of single amino acid $\mathrm{Na}_{\mathrm{v}} \mathrm{l} .7$ variants, with biophysical properties different than those of $\mathrm{Na}_{\mathrm{v}} \mathrm{l} .7$ IEM or PEPD mutations, in nearly one-third of patients with I-SFN. ${ }^{14}$ All of these variants produced hyperexcitability within DRG neurons. After expression within HEK293 cells, we observed impaired slow-inactivation for 1 of those variants $(\mathrm{I} 2 \mathrm{OK}){ }^{14}$ Ion channels can, however, display different functional properties within different cell backgrounds, and biophysical changes cannot be detected in some $\mathrm{Na}_{\mathrm{v}} 1.7$ mutations after expression within heterologous systems such as the nonneuronal HEK293 cells, but are demonstrable after expression within native DRG neurons. ${ }^{23,24}$ In this study, we demonstrate that the $\mathrm{Na}_{\mathrm{v}} \mathrm{T} .7 / \mathrm{l} 739 \mathrm{~V}$ variant, in a patient with skin biopsy- and QST-confirmed I-SFN, impairs slow-inactivation after expression within DRG neurons. We also show that I739V channels render 
Figure 4. $1739 \mathrm{~V}$ channels produce hyperexcitability in dorsal root ganglion (DRG) neurons

A
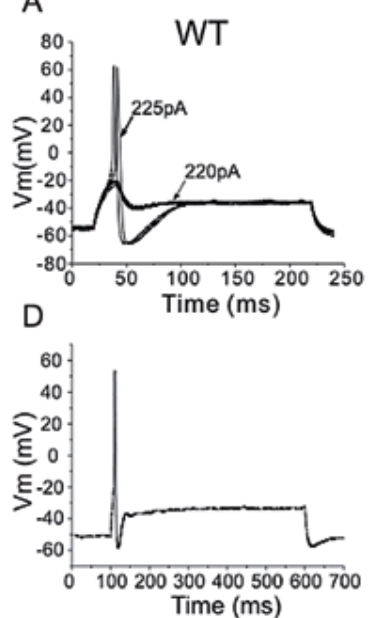

G

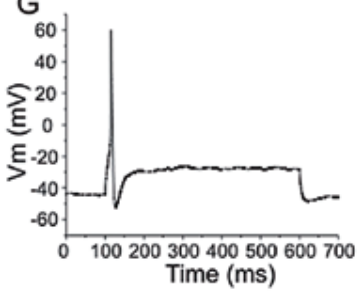

B
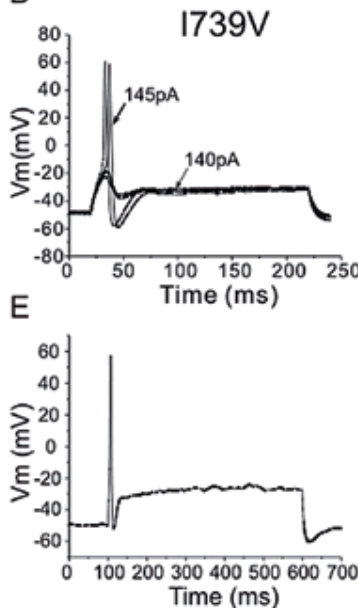

$\mathrm{H}$

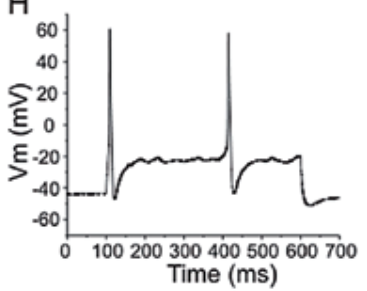

$\mathrm{C}$

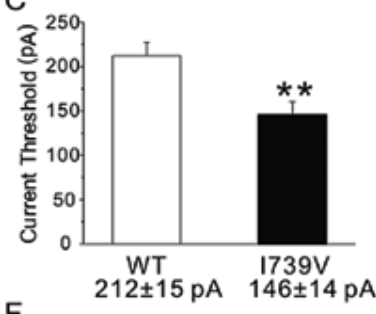

$\mathrm{F}$
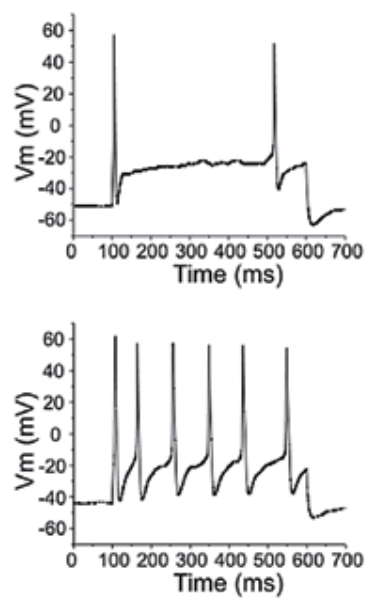

$J$

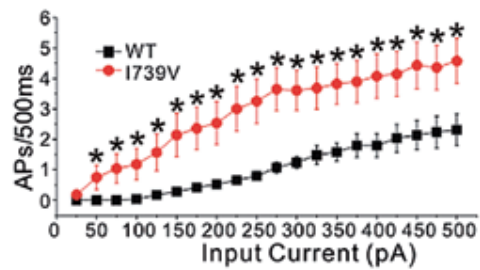

Legend to figure 4. Current-clamp analysis of DRG neurons expressing $1739 \mathrm{~V}$ channels. Representative action potential traces recorded from DRG neuron expressing wild-type (A) or I739V (B) channels, respectively. Action potentials were elicited by 200-msec step depolarizing current injections. (C) Comparison of current threshold for DRG neurons expressing wild-type and $1739 \mathrm{~V}$ mutant channels. Current threshold of cells transfected with $1739 \mathrm{~V}(146 \pm 14 \mathrm{pA}$, $n=28$; $* * 0.01)$ was significantly smaller than wild-type channels $(212 \pm 15 p A, n=29)$. (D-F) Responses of a representative DRG neuron expressing wild-type channels to 500 msec depolarization current steps at $1 \times(D), 1.5 \times(E)$, and $2 \times(F)$ the current threshold of this neuron. $(G-I)$ Responses of a representative DRG neuron expressing $1739 \mathrm{~V}$ mutant channels to $500 \mathrm{msec}$ depolarization current steps at $1 \times(\mathrm{G}), 1.5 \times(\mathrm{H})$, and $2 \times(\mathrm{I})$ the current threshold of the cell. (J) Comparison of action potential spike numbers between DRG neurons expressing wild-type and $1739 \mathrm{~V}$ across a range of $500-\mathrm{msec}$ step current injections from 25 to $500 \mathrm{pA}$ ( $* \mathrm{p}<0.05)$. 
small DRG neurons, which give rise to small-diameter peripheral axons, hyperexcitable.

Gain-of-function $\mathrm{Na}_{\mathrm{v}} \mathrm{l} .7$ mutations have been previously linked to IEM, characterized by distal burning pain" and PEPD, characterized by perirectal, periocular, and perimandibular pain. ${ }^{3}$ The present results demonstrate a $\mathrm{Na}_{\mathrm{v}}{ }^{1} .7$ variant, with a gain-of-function profile not previously linked to IEM or PEPD, i.e., isolated impairment of slowinactivation, in a patient with I-SFN. The clinical picture in this patient, however, differed from that in IEM and PEPD: first, the patient described here displayed profound autonomic symptoms; autonomic symptoms are not prominent in IEM except for skin reddening during attacks." ${ }^{29}$ Second, there was pain in the face, scalp, and hair, as well as lower legs and feet, and in the joints, in the patient we describe; in contrast, pain in IEM is mainly located in the distal extremities. Interestingly, the $\mathrm{Na}_{\mathrm{v}} 1.7 / 1739 \mathrm{~V}$ variant produced hyperexcitability in DRG neurons as previously described for IEM mutations, ${ }^{11,12}$ but impaired slow-inactivation without the hyperpolarized activation and enhanced ramp responses characteristic of IEM mutations" or incomplete fast-inactivation ${ }^{13}$ characteristic of PEPD mutations.

The 8 patients with I-SFN described by Faber et al. ${ }^{14}$ developed clinical signs of neuropathy, including neuropathic pain, in adulthood, with age at onset ranging from 14 (present patient) to 68 years, while housing $\mathrm{Na}_{\mathrm{v}} 1.7$ variants throughout their lives. A previous study ${ }^{30}$ reported $\mathrm{Na}_{\mathrm{v}} \mathrm{1} \cdot 7 / 1739 \mathrm{~V}$ in 1 patient with febrile seizures, 1 patient with Dravet syndrome, and $1 / 562$ controls; we found the $1739 \mathrm{~V}$ variant in $1.4 \%$ of control DNA samples. Neither the patient described here, nor patients housing gain-of-function variants of $\mathrm{Na}_{\mathrm{V}}{ }^{1.7}$ associated with IEM ${ }^{11,29}$ or $\mathrm{SFN},{ }^{14}$ have reported seizures. Whether this sodium channel variant increases risk of development of SFN, in individuals housing modifier genes or exposed to triggering factors, is not clear, and whether these apparently normal controls would have developed SFN or any other hyperexcitability disorder later in life is not known. Triggering factors and other mechanisms responsible for the timedependent onset of signs and symptoms of I-SFN might include epigenetic, activity-dependent, or environmental factors, and remain to be determined.

1739 is conserved among $\mathrm{Na}_{\mathrm{v}} \mathrm{T} .7$ mammalian orthologues and human channel paralogues and we observed altered gating of $1739 \mathrm{~V}$. Nevertheless, as noted above, this variant is present in $1.4 \%$ of control DNA samples, raising the question of whether it is functionally significant. Missense substitutions in DII/S1 of sodium channels, while not functionally profiled, have been linked to Dravet syndrome $\left(\mathrm{Na}_{\mathrm{v}} \mathrm{T} .1 / \mathrm{L} 783 \mathrm{P}\right)$ and sudden unexplained nocturnal death syndrome $\left(\mathrm{Na}_{\mathrm{v}} 1.5 / \mathrm{A} 735 \mathrm{~V}\right) .^{31}{ }^{32} \mathrm{Na}_{\mathrm{v}}{ }^{1.5} / \mathrm{A} 735 \mathrm{~V}$, located near the extracellular end of $\mathrm{DII} / \mathrm{S}_{1}$, was reported to depolarize activation. ${ }^{32}$ In contrast, $\mathrm{Na}_{\mathrm{v}} \mathrm{1} .7 / \mathrm{l} 739 \mathrm{~V}$, within the middle of DII/S1, impairs slow-inactivation. Multiple missense mutations impair or enhance slow-inactivation of $\mathrm{Na}_{\mathrm{v}} 1 \cdot 7,{ }^{12}$ and a unifying mechanism has 
not been identified. Given that $1739 \mathrm{~V}$ represents a conservative change, size of the side chain may impact packing of the DII/Si a-helical structure, possibly affecting slow-inactivation.

Although a definitive demonstration of a causative link of $1739 \mathrm{~V}$ substitution to SFN will require study of larger kindreds, including clinical tracking of affected status with base alterations, our results clearly demonstrate that expression of $1739 \mathrm{~V}$ channels increases DRG neuron excitability. Our results are supported by earlier computer simulations which suggest that changes in slow-inactivation can modulate DRG neuron excitability. ${ }^{33}$ A recent study demonstrated experimentally that, indeed, enhanced slow-inactivation can partially offset the DRG neuron hyperexcitability induced by hyperpolarized activation by an IEM mutation, thereby modulating clinical phenotype. ${ }^{34}$ While we cannot rule out the possibility that $1739 \mathrm{~V}$ alters activation, fast-inactivation, persistent, resurgent, or ramp currents-all implicated in neuronal hyperexcitability-in human as compared to rodent DRG neurons, the most parsimonious interpretation of our data is that $1739 \mathrm{~V}$-induced impairment of slow-inactivation of $\mathrm{Na}_{\mathrm{v}} 1.7$ causes DRG neuron hyperexcitability.

While a causal link between $1739 \mathrm{~V}$ and axonal degeneration has not been experimentally demonstrated, several lines of evidence suggest a role of sodium channels in axonal injury. $\mathrm{Na}_{\mathrm{v}} \mathrm{T} .7$, which activates in response to small depolarizations close to resting potential, ${ }^{10}$ is coexpressed within smalldiameter peripheral axons together with $\mathrm{Na}_{\mathrm{v}} 1.6, \mathrm{Na}_{\mathrm{v}} 1.8$, and $\mathrm{Na}_{\mathrm{v}} 1.9$ sodium channels and the $\mathrm{NCX}_{2}$ sodium-calcium exchanger. ${ }^{9}$ Activity of sodium channels can injure axons via calcium-importing reverse sodium-calcium exchange if the ability to extrude sodium is exceeded. ${ }^{35}$ Moreover, impulse activity at physiologic frequencies can sensitize axons to otherwise reversible metabolic insults, triggering axonal degeneration ${ }^{36}$ that can be ameliorated by sodium channel blockers. ${ }^{37}$ Increased $\mathrm{Na}^{+}$influx can impose an especially large energetic load in small-diameter axons, due to high surface-to-volume ratio and input resistance, low capacitance per unit length, and shorter length constant. ${ }^{38,} 39$ Hypoxic neuropathy has, in fact, been demonstrated to produce degeneration of small diameter nonmyelinated axons..$^{40}$ Future studies will be needed to determine whether $1739 \mathrm{~V}$ produces or predisposes to axonal degeneration.

Our results, from a patient with painful biopsy and QST-confirmed idiopathic small fiber neuropathy, provide support for the suggestion that $\mathrm{Na}_{\mathrm{v}} 1.7$ variants that impair channel slow-inactivation can contribute to pain in SFN by increasing nociceptor excitability. Future studies will hopefully resolve the question of whether gain-of-function variants of $\mathrm{Na}_{\mathrm{v}} \mathrm{T} .7$ contribute to degeneration of small nerve fiber. 


\section{References}

1. Devigili, G. et al. The diagnostic criteria for small fibre neuropathy: from symptoms to neuropathology. Brain 131, 1912-25 (2008).

2. Gorson, K.C. \& Ropper, A.H. Idiopathic distal small fiber neuropathy. Acta Neurol Scand 92, 376-82 (1995).

3. Lacomis, D. Small-fiber neuropathy. Muscle Nerve 26, 173-88 (2002).

4. Holland, N.R. et al. Small-fiber sensory neuropathies: clinical course and neuropathology of idiopathic cases. Ann Neurol 44, 47-59 (1998).

5. Stewart, J.D., Low, P.A. \& Fealey, R.D. Distal small fiber neuropathy: results of tests of sweating and autonomic cardiovascular reflexes. Muscle Nerve 15, 661-5 (1992).

6. Bednarik, J. et al. Etiology of small-fiber neuropathy. J Peripher Nerv Syst 14, 177-83 (2009).

7. Toledo-Aral, J.J. et al. Identification of $\mathrm{PN}$, a predominant voltage-dependent sodium channel expressed principally in peripheral neurons. Proc Natl Acad Sci U S A 94, 1527-32 (1997).

8. Rush, A.M. et al. A single sodium channel mutation produces hyper- or hypoexcitability in different types of neurons. Proc Natl Acad Sci U S A 103, 8245-50 (2006).

9. Persson, A.K. et al. Sodium-calcium exchanger and multiple sodium channel isoforms in intra-epidermal nerve terminals. Mol Pain 6, 84 (2010).

10. Cummins, T.R., Howe, J.R. \& Waxman, S.G. Slow closed-state inactivation: a novel mechanism underlying ramp currents in cells expressing the hNE/PN1 sodium channel. J Neurosci 18, 9607-19 (1998).

11. Dib-Hajj, S.D., Cummins, T.R., Black, J.A. \& Waxman, S.G. Sodium channels in normal and pathological pain. Annu Rev Neurosci 33, 325-47 (2010).

12. Dib-Hajj, S.D. et al. Gain-of-function mutation in Nav1.7 in familial erythromelalgia induces bursting of sensory neurons. Brain 128, 1847-54 (2005).

13. Fertleman, C.R. et al. SCNgA mutations in paroxysmal extreme pain disorder: allelic variants underlie distinct channel defects and phenotypes. Neuron 52, 767-74 (2006).

14. Faber, C.G. et al. Gain of function $\mathrm{Na}(\mathrm{V}) 1.7$ mutations in idiopathic small fiber neuropathy. Ann Neurol 7 , 26-39 (2012).

15. Lauria, G. et al. European Federation of Neurological Societies/Peripheral Nerve Society Guideline on the use of skin biopsy in the diagnosis of small fiber neuropathy. Report of a joint task force of the European Federation of Neurological Societies and the Peripheral Nerve Society. Eur J Neurol 17, 903-12, e44-9 (2010)

16. Bakkers, M. et al. Intraepidermal nerve fiber density and its application in sarcoidosis. Neurology 73, 11428 (2009).

17. Shy, M.E. et al. Quantitative sensory testing: report of the Therapeutics and Technology Assessment Subcommittee of the American Academy of Neurology. Neurology 60, 898-904 (2003).

18. Reulen, J.P., Lansbergen, M.D., Verstraete, E. \& Spaans, F. Comparison of thermal threshold tests to assess small nerve fiber function: limits vs. levels. Clin Neurophysiol 114, 556-63 (2003).

19. Yarnitsky, D. \& Sprecher, E. Thermal testing: normative data and repeatability for various test algorithms. J Neurol Sci 125, 39-45 (1994).

20. Hoitsma, E. et al. Abnormal warm and cold sensation thresholds suggestive of small-fibre neuropathy in sarcoidosis. Clin Neurophysiol 114, 2326-33 (2003).

21. Drenth, J.P. et al. SCNgA mutations define primary erythermalgia as a neuropathic disorder of voltage gated sodium channels. J Invest Dermatol 124, 1333-8 (2005).

22. Klugbauer, N., Lacinova, L., Flockerzi, V. \& Hofmann, F. Structure and functional expression of a new member of the tetrodotoxin-sensitive voltage-activated sodium channel family from human neuroendocrine cells. EMBOJ 14, 1084-90 (1995).

23. Cummins, T.R., Rush, A.M., Estacion, M., Dib-Hajj, S.D. \& Waxman, S.G. Voltage-clamp and currentclamp recordings from mammalian DRG neurons. Nat Protoc 4, 1103-12 (2009).

24. Dib-Hajj, S.D. et al. Transfection of rat or mouse neurons by biolistics or electroporation. Nat Protoc 4, 1118-26 (2009).

25. Wellmann, H., Kaltschmidt, B. \& Kaltschmidt, C. Optimized protocol for biolistic transfection of brain slices and dissociated cultured neurons with a hand-held gene gun.J Neurosci Methods 92, 55-64 (1999).

26. Nassar, M.A. et al. Nociceptor-specific gene deletion reveals a major role for $\mathrm{Nav1.7}(\mathrm{PN} 1)$ in acute and inflammatory pain. Proc Natl Acad Sci U S A 101, 12706-11 (2004). 
27. Herzog, R.I., Cummins, T.R., Ghassemi, F., Dib-Hajj, S.D. \& Waxman, S.G. Distinct repriming and closedstate inactivation kinetics of Nav1.6 and Nav1.7 sodium channels in mouse spinal sensory neurons. J Physiol 551, 741-50 (2003).

28. Han, C. et al. Early- and late-onset inherited erythromelalgia: genotype-phenotype correlation. Brain $\mathbf{1 3 2}$, 1711-22 (2009).

29. Drenth, J.P. \& Waxman, S.G. Mutations in sodium-channel gene SCNgA cause a spectrum of human genetic pain disorders. J Clin Invest 117, 3603-9 (2007).

30. Singh, N.A. et al. A role of $\mathrm{SCNgA}$ in human epilepsies, as a cause of febrile seizures and as a potential modifier of Dravet syndrome. PLoS Genet 5, elooo649 (2009).

31. Harkin, L.A. et al. The spectrum of SCN1A-related infantile epileptic encephalopathies. Brain 130, $843-52$ (2007)

32. Vatta, M. et al. Genetic and biophysical basis of sudden unexplained nocturnal death syndrome (SUNDS), a disease allelic to Brugada syndrome. Hum Mol Genet 11, 337-45 (2002).

33. Sheets, P.L., Jackson, J.O., 2nd, Waxman, S.G., Dib-Hajj, S.D. \& Cummins, T.R. A Nav1.7 channel mutation associated with hereditary erythromelalgia contributes to neuronal hyperexcitability and displays reduced lidocaine sensitivity. J Physiol 581, 1019-31 (2007).

34. Cheng, $\mathrm{X}$. et al. Deletion mutation of sodium channel $\mathrm{Na}(\mathrm{V}) 1.7$ in inherited erythromelalgia: enhanced slow inactivation modulates dorsal root ganglion neuron hyperexcitability. Brain 134, 1972-86 (2011).

35. Stys, P.K., Waxman, S.G. \& Ransom, B.R. Na(+)-Ca2+ exchanger mediates Ca2+ influx during anoxia in mammalian central nervous system white matter. Ann Neurol 30, 375-80 (1991).

36. Smith, K.J., Kapoor, R., Hall, S.M. \& Davies, M. Electrically active axons degenerate when exposed to nitric oxide. Ann Neurol 49, 470-6 (2001).

37. Kapoor, R., Davies, M., Blaker, P.A., Hall, S.M. \& Smith, K.J. Blockers of sodium and calcium entry protect axons from nitric oxide-mediated degeneration. Ann Neurol 53, 174-80 (2003).

38. Waxman, S.G., Black, J.A., Kocsis, J.D. \& Ritchie, J.M. Low density of sodium channels supports action potential conduction in axons of neonatal rat optic nerve. Proc Natl Acad Sci U S A 86, 1406-10 (1989).

39. Donnelly, D.F. Spontaneous action potential generation due to persistent sodium channel currents in simulated carotid body afferent fibers. J Appl Physiol 104, 1394-401 (2008).

40. Malik, R.A. et al. Hypoxic neuropathy: relevance to human diabetic neuropathy. Diabetologia $33,311-8$ (1990). 

Chapter 6

\section{Functional profiles of $S C N g A$}

\section{variants in dorsal root}

\section{ganglion neurons and superior} cervical ganglion neurons correlate with autonomic symptoms in small fiber neuropathy

C. Han ${ }^{a, b}$, J.G.J. Hoeijmakers ${ }^{c}$, S. Liua, ${ }^{a, b}$, M.M. Gerrits ${ }^{d}$, R.H.M. te Morsche, G. Lauriaf, S.D. Dib-Hajja, , J.P.H. Drenthf, C.G. Faber, I.S.J. Merkies, ${ }^{c, g}$, S.G. Waxman ${ }^{a, b}$.

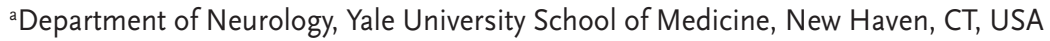
${ }^{b}$ Center for Neuroscience and Regeneration Research, Veterans Affairs Medical Center,

West Haven, CT, USA

'Department of Neurology, Maastricht University Medical Center, Maastricht

${ }^{\mathrm{d}}$ Department of Clinical Genomics, Maastricht University Medical Center, Maastricht ${ }^{e}$ Department of Gastroenterology and Hepatology, Radboud University Nijmegen Medical

Center, Nijmegen

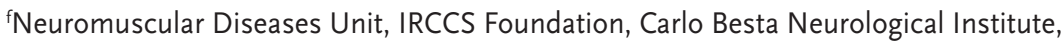
Milan, Italy

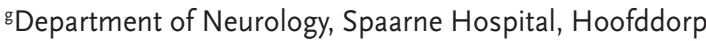

Brain. 2012;135(Pt 9):2613-28; Published with permission from Oxford Journals 


\begin{abstract}
Patients with small fiber neuropathy typically manifest pain in distal extremities and severe autonomic dysfunction. However, occasionally patients present with minimal autonomic symptoms. The basis for this phenotypic difference is not understood. Sodium channel $\mathrm{Na}_{\mathrm{v}} \mathrm{T} .7$, encoded by the $S C N g A$ gene, is preferentially expressed in the peripheral nervous system within sensory dorsal root ganglion and sympathetic ganglion neurons and their small diameter peripheral axons. We recently reported missense substitutions in SCNgA that encode functional $\mathrm{Na}_{\mathrm{v}} \mathrm{T} .7$ variants in $28 \%$ of patients with biopsy-confirmed small fiber neuropathy. Two patients with biopsy-confirmed small fiber neuropathy manifested minimal autonomic dysfunction unlike the other six patients in this series, and both of these patients carry the $\mathrm{Na}_{v} 1.7 / \mathrm{R}_{1} 85 \mathrm{H}$ variant, presenting the opportunity to compare variants associated with extreme ends of a spectrum from minimal to severe autonomic dysfunction. Herein, we show by voltageclamp that $\mathrm{R} 185 \mathrm{H}$ variant channels enhance resurgent currents within dorsal root ganglion neurons and show by current-clamp that $\mathrm{R} 185 \mathrm{H}$ renders dorsal root ganglion neurons hyperexcitable. We also show that in contrast, $\mathrm{R} 185 \mathrm{H}$ variant channels do not produce detectable changes when studied by voltage-clamp within sympathetic neurons of the superior cervical ganglion, and have no effect on the excitability of these cells. As a comparator, we studied the $\mathrm{Na}_{\mathrm{v}} 1.7$ variant $1739 \mathrm{~V}$, identified in three patients with small fiber neuropathy characterized by severe autonomic dysfunction as well as neuropathic pain, and show that this variant impairs channel slow inactivation within both dorsal root ganglion and superior cervical ganglion neurons, and renders dorsal root ganglion neurons hyperexcitable and superior cervical ganglion neurons hypoexcitable. Thus, we show that $\mathrm{R} 185 \mathrm{H}$, from patients with minimal autonomic dysfunction, does not produce detectable changes in the properties of sympathetic ganglion neurons, while $1739 \mathrm{~V}$, from patients with severe autonomic dysfunction, has a profound effect on excitability of sympathetic ganglion neurons.
\end{abstract}




\section{Introduction}

Small fiber neuropathy, a disorder typically manifested as neuropathic pain in distal extremities and profound autonomic symptoms, is characterized by degeneration of epidermal nerve endings of thinly myelinated and unmyelinated nerve fibers. ${ }^{1-6}$ Small fiber neuropathy has been reported to be idiopathic, i.e. does not have an identifiable cause, in $24-93 \%$ of patients in various series. ${ }^{4,6,7}$ Recently, we reported variants of voltage-gated sodium channel $\mathrm{Na}_{\mathrm{v}} \mathrm{T} \cdot 7$, encoded by the $S C N_{9} A$ gene, in $28 \%$ of patients with biopsy-confirmed idiopathic small fiber neuropathy. ${ }^{8}$

Voltage-gated sodium channel $\mathrm{Na}_{\mathrm{v}} 1.7$, encoded by the $S C N_{9} A$ gene, is abundantly present in dorsal root ganglion (DRG) and sympathetic ganglion neurons, ${ }^{9} 10$ and their axons." $\mathrm{Na}_{\mathrm{v}} 1.7$ opens in response to small depolarizations close to resting potential. ${ }^{12}$ Gain-of-function mutations in $\mathrm{Na}_{\mathrm{v}} 1.7$ that enhance activation and impair fast inactivation cause the heritable painful disorders inherited erythromelalgia ${ }^{13}, 14$ and paroxysmal extreme pain disorder. ${ }^{15}$ The channel variants from patients with $\mathrm{Na}_{\mathrm{v}} 1.7$ channelopathy-associated small fiber neuropathy that have been investigated functionally thus far show gainof-function properties, impaired fast-inactivation, impaired slow-inactivation or enhanced resurgent current and render DRG neurons hyperexcitable, a change that is thought to underlie the neuropathic pain reported by patients carrying these variants..$^{8,16}$

Despite the debilitating nature of autonomic symptoms in small fiber neuropathy, the molecular basis for autonomic dysfunction in small fiber neuropathy is not well understood. Most patients with $\mathrm{Na}_{\mathrm{v}} \mathrm{l} .7$ channelopathy-associated small fiber neuropathy experience profound autonomic dysfunction in addition to neuropathic pain. ${ }^{8}$ However, small fiber neuropathy can sometimes present a picture of pain and sensory dysfunction with only minimal autonomic dysfunction. Two of the eight patients described in the previous paper by Faber et al. (2012) reported minimal autonomic symptomatology, and both carried the $\mathrm{R} 185 \mathrm{H}$ variant $\mathrm{Na}_{\mathrm{v}} 1.7$ channel. Although all the variants studied thus far have produced hyperexcitability within DRG neurons, consistent with the pain phenotype in these patients, the effects of these variants on sympathetic neurons, which might correlate with autonomic dysfunction, have not been investigated. Reasoning that $\mathrm{R} 185 \mathrm{H}$ channels might affect DRG and sympathetic neurons in different ways, we assessed the biophysical effects of this variant by voltage-clamp in both DRG and sympathetic ganglion neurons [superior cervical ganglia (SCG)] and examined the functional effects of this channel on firing properties of neurons from DRG and SCG. As a comparator, we assessed $\mathrm{Na}_{\mathrm{v}} \mathrm{T} .7 / \mathrm{l} 339 \mathrm{~V}$, the variant channel associated with the most severe autonomic symptoms in our series. ${ }^{8}$ 


\section{Materials and methods}

\section{Patients}

The patients, three of whom were evaluated as part of a series reported by Faber et al. (2012), and two additional patients evaluated subsequently at Maastricht University Medical Centre neurological clinic, were diagnosed with small fiber neuropathy. This study was approved by medical ethics committees at Yale University and Maastricht University Medical Center. All aspects of the study were explained and written informed consent obtained prior to study initiation.

\section{Clinical characterization}

Skin biopsy

Punch biopsy (10 cm above lateral malleolus) specimens were fixed (2\% paraformaldehyde-lysine-sodium periodate, $4^{\circ} \mathrm{C}$ ), cryoprotected and stored $\left(-80^{\circ} \mathrm{C}\right)$ in $20 \%$ glycerol before sectioning $(50 \mu \mathrm{m})$. Numbers of individual nerve fibers crossing dermal-epidermal junctions were analyzed in each of three sections, immunostained with polyclonal rabbit anti-protein gene product 9.5 antibody (PGP9.5; Ultraclone), by bright-field microscopy (Olympus BX 50 stereology workstation, PlanApo oil objective 40x/numerical aperture $=1.0$ ). Linear quantification of intraepidermal nerve fiber density (IENF/mm) was compared with age- and gender-adjusted normative values. ${ }^{17}$

\section{Quantitative sensory testing}

Quantitative sensory testing was performed according to previous guidelines ${ }^{18}$ with a TSA-2001 instrument (Medoc). Thresholds were assessed at the dorsum of both feet and thenar eminences, using ascending/descending (warm/cool) thermal ramp stimuli. Heat pain modality was also examined. Results were compared with normative values,,$^{19}$ and were considered abnormal for Z-values $>2.5$. Sensory modalities were classified as abnormal if results of both methodof-limits and method-of-levels were abnormal. ${ }^{20}$

\section{SCNgA sequence analysis}

Genomic DNA isolation and amplification/analysis of SCNgA-coding exons and flanking intronic sequences, and exons encoding $5^{\prime}$ and $3^{\prime}$ untranslated sequences within complementary DNA, were described previously. ${ }^{21}$ Exon sequences were compared with reference $\mathrm{Na}_{\mathrm{v}} 1.7$ complementary DNA (NM $002977.3)^{22}$ to identify variations using Alamut mutation interpretation software (Interactive-Biosoftware). DNA from 1000 Caucasian control subjects (2000 chromosomes) from the same geographical region as the index patient was analysed for $\mathrm{C} .554 \mathrm{G}>\mathrm{A}$ and $\mathrm{C.2215} \mathrm{A}>\mathrm{C}$ substitutions by $\mathrm{PCR}$ and high-resolution melting curve analysis. Primer pairs $3^{\prime} A T G G T C G T T G T A T T C T T T T C A 5^{\prime} / 3^{\prime}-$ 
AACACTGTGCTGCCTGAG5' and 3'TTTTATTGTAATGGATCCTTTTGTAG5'/3'AT TGGGTCGTGTT CCATAGC5', and fluorescent dye EvaGreen (C) (Biotium) were used to amplify the variant exons; presence of variant alleles was determined from melting curves $\left(65^{\circ} \mathrm{C}\right.$ to $95^{\circ} \mathrm{C}$; ramp rate $0.1^{\circ} \mathrm{C} / 10 \mathrm{~s}$; CFX96TM RealTime PCR Detection System), analysed using Precision Melt AnalysisTM software (Bio-Rad). We interrogated two online databases (the 1000 genome project; www.10oogenomes.org) and the Exome Variant Database (http:// evs.gs.washington.edu/EVS) for the presence of detected variants in control populations.

\section{Functional analysis}

Transfection of dorsal root ganglion and superior cervical ganglion neurons and voltage-clamp recordings

All protocols for care and sacrifice of animals were approved by the Veterans Administration Connecticut IACUC. Since initial voltage-clamp assessment did not reveal biophysical changes in properties of R185 $\mathrm{H}$ channels expressed within HEK293 cells, voltage-clamp analysis was performed after biolistic (Helios (C) Gene Gun, Bio-Rad) transfection of DRG cultured from 4 - to 8-weekold $\mathrm{Na}_{\mathrm{v}} 1.8$-null mice ${ }^{23}$ as described previously. ${ }^{24}$ Wild-type or $\mathrm{R}_{1} 85 \mathrm{H}$ plasmids were mixed with green fluorescent protein (GFP) plasmid DNA (channel:GFP ratio 3:1) using $1.0 \mu \mathrm{m}$ gold particles, discharged into cells at a pressure of $\sim 120$ psi $(\sim 827 \mathrm{kPa})$. Absence of $\mathrm{Na}_{\mathrm{v}} 1.8$ tetrodotoxin-resistant current in these DRG neurons facilitated analysis of wild-type and $\mathrm{R}_{1} 85 \mathrm{H} \mathrm{Na}_{\mathrm{v}} 1.7$ channels, which were rendered tetrodotoxin resistant. ${ }^{25}$

Adult $\mathrm{Na}_{\mathrm{v}} 1.8$-null mice or rats did notyield SCG neurons that could be reproducibly transfected with sodium channel constructs. Therefore, we used rat pups as the source for SCG neurons for transfection. SCG were harvested from o- to 5-dayold Sprague-Dawley rats, and neurons were isolated as previously described. ${ }^{\circ}$ SCG neurons were maintained under standard tissue culture conditions for $\sim 8 \mathrm{~h}$ before biolistic transfections. Tetrodotoxin resistant versions of wild-type, $\mathrm{R} 185 \mathrm{H}$ or $1739 \mathrm{~V}$ plasmids were mixed with GFP plasmid DNA (channel:GFP ratio 3:1) using $1.0 \mu \mathrm{m}$ gold particles, discharged into cells at a pressure of $\sim 240$ psi $(\sim 1654 \mathrm{kPa})$. Since tetrodotoxin resistant sodium currents are absent in SCG neurons, the tetrodotoxin resistant ${ }^{25}$ versions of $\mathrm{Na}_{\mathrm{v}} 1.7$ wild-type, $\mathrm{R}_{1} 85 \mathrm{H}$ or $1739 \mathrm{~V}$ facilitated the functional analysis of these channels.

Whole-cell patch-clamp recordings in DRG or SCG neurons were obtained in voltage-clamp mode at room temperature $\left(\sim 21^{\circ} \mathrm{C}\right), 4 \mathrm{O}-48 \mathrm{~h}$ after transfection using 0.8-1.5M $\Omega$ electrodes and EPC-9 amplifier and Pulse 8.5 (HEKA). Cells transfected with wild-type or variant $\mathrm{Na}_{\mathrm{v}} 1.7$ channels were selected using identical criteria, which required that cells be GFP-fluorescent, isolated, round with 20-30 $\mu \mathrm{m}$ diameter and one or fewer neurites (to reduce space- 
clamp artefact), smooth membranes, peak current $>\ln A$. Voltage-errors were minimized using 80-90\% series resistance-compensation and linear leak subtraction; capacitance artefact was cancelled using computer-controlled circuitry. The pipette solution contained (mM): $140 \mathrm{CsF} / 1 \mathrm{EGTA} / 10 \mathrm{NaCl} / 10$ HEPES/ pH 7.3 with $\mathrm{CsOH}$ (adjusted to $315 \mathrm{mmol} / \mathrm{L}$ with dextrose). The bath solution was $(\mathrm{mM}) 140 \mathrm{NaCl} / 3 \mathrm{KCl} / 1 \quad \mathrm{MgCl}_{2} / 1 \mathrm{CaCl}_{2} / 20 \mathrm{TEA}-\mathrm{Cl} / 5 \mathrm{CsCl} / 0.1$ $\mathrm{CdCl}_{2} / 0.0003$ tetrodotoxin/10 HEPES, $\mathrm{pH} 7.3$ with $\mathrm{NaOH}$ (adjusted to 320 $\mathrm{mmol} / \mathrm{L}$ with dextrose).

Recordings were started 5 min after establishing whole-cell configuration. DRG or SCG neurons were held at $-100 \mathrm{mV}$ and stepped to a range of potentials $(-80$ to $+60 \mathrm{mV}$ in $5 \mathrm{mV}$ increments) for $100 \mathrm{~ms}$. Peak inward currents $(I)$ were plotted as a function of depolarization potential to generate $I-V$ curves. Activation curves were obtained by converting I to conductance $(G)$ at each voltage $(V)$ using the equation $G=I /\left(V-V_{\text {rev }}\right)$, where $V_{\text {rev }}$ is the reversal potential which was determined for each cell individually. Activation curves were then fit with Boltzmann functions in the form of $G=G_{\max } /\left(1+\exp \left[\left(V_{1 / 2, a c t-v}\right) / k\right]\right\}$, where $G_{\text {max }}$ is the maximal sodium conductance, $V_{1 / 2, a c t}$ is the potential at which activation is half-maximal, $\mathrm{V}$ is the test potential and $k$ is the slope factor. Steady-state fastinactivation was examined using a series of $500 \mathrm{~ms}$ prepulses from -150 to omV followed by test pulses to $-10 \mathrm{mV}$. Steady-state slow-inactivation was assessed using a $20 \mathrm{~ms}$ pulse to $-10 \mathrm{mV}$ after a $30 \mathrm{~s}$ prepulse to potentials ranging from -130 to $10 \mathrm{mV}$ followed by a $100 \mathrm{~ms}$ pulse to $-120 \mathrm{mV}$ to remove fast-inactivation. Peak inward currents obtained from steady-state fast inactivation and slow inactivation protocols were normalized to the maximal peak current (Imax) and fit with Boltzmann functions:

$\left.I / I_{\max }=1 /\left(1+\exp \left[V-V_{1 / 2, \text { inact }}\right) / \mathrm{k}\right]\right\}$ for fast inactivation, and $I / I_{\max }=R_{\text {in }}+\left(1-R_{\text {in }}\right) /$ $\left.\left\{1+\exp \left[V-V_{1 / 2, \text { inact }}\right) / k\right]\right\}$ for slow inactivation, where $V$ represents the inactivating prepulse potential, $V_{1 / 2}$, inact represents the midpoint of the inactivation curve and $R_{\text {in }}$ is the fraction of channels that are resistant to inactivation. To assess deactivation, cells were held at $-100 \mathrm{mV}$ and tail currents generated by $0.5 \mathrm{~ms}$ depolarization to $-20 \mathrm{mV}$ followed by a series of repolarizations (-100 to $-40 \mathrm{mV}$ ). Deactivation kinetics was measured using single exponential fits to estimate current decay time at potentials from -100 to $-40 \mathrm{mV}$.

Resurgent currents were assessed with a two-step protocol that initially depolarized the membrane to $+30 \mathrm{mV}$ for $20 \mathrm{~ms}$ before testing for resurgent sodium currents by hyperpolarizing the membrane potential in $-5 \mathrm{mV}$ increments from o to $-80 \mathrm{mV}$ for $100 \mathrm{~ms}$, then returning to the holding potential of -100 $\mathrm{mV}$. Recordings for resurgent current reveal two components: transient peak current evoked by the $30 \mathrm{mV}$ depolarization and the following resurgent current by repolarization. The peak current may display a small outward current at the end of $20 \mathrm{~ms}$ recording, which is due to leakage. The relative amplitude 
of the resurgent current was calculated as a percentage of the peak current evoked by a $+30 m V$ depolarization. Quantification and analysis of resurgent sodium current were carried out as described previously. ${ }^{26}$ Under our recording conditions, we were able to reliably detect resurgent currents with amplitude $>100 \mathrm{pA}$ and could detect resurgent currents as small as $50 \mathrm{pA}$ if they displayed typical waveform in cells with low noise.

Transfection of dorsal root ganglion and superior cervical ganglion neurons: currentclamp recordings

SCG neurons were isolated and cultured from o - to 5-day-old Sprague-Dawley rats as described previously. ${ }^{10}$ DRG neurons from animals of the same age were cultured using a protocol as described by Dib-hajj et al. (2009). Wild-type $\mathrm{Na}_{\mathrm{V}} 1.7_{\mathrm{R}}, \mathrm{R} 185 \mathrm{H}$, or $1739 \mathrm{~V}$ variant channels and GFP constructs (channel:GFP ratio 5:1) were electroporated into DRG or SCG neurons using Rat Neuron Nucleofector @ Solution (Lonza) as described previously. ${ }^{3}$ Whole-cell currentclamp recordings were obtained from transfected small diameter $(<25 \mu \mathrm{m})$ DRG or SCG neurons with robust GFP fluorescence, within $40-48 \mathrm{~h}$ by using an EPC-9 amplifier and Pulse 8.5 (HEKA). Electrodes $(1-3 \mathrm{M} \Omega)$ were filled with pipette solution $(\mathrm{mM}): 140 \mathrm{KCl} / 0.5 \mathrm{EGTA} / 5 \mathrm{HEPES} / 3 \mathrm{Mg}-\mathrm{ATP} / \mathrm{pH} 7.3$ with $\mathrm{KOH}$ (adjusted to $315 \mathrm{mmol} / \mathrm{L}$ with dextrose). The extracellular solution contained (mM): $140 \mathrm{NaCl} / 3 \mathrm{KCl} / 2 \mathrm{MgCl}_{2} / 2 \mathrm{CaCl}_{2} / 10 \mathrm{HEPES}, \mathrm{pH} 7.3$ with $\mathrm{NaOH}$ (adjusted to $320 \mathrm{mmol} / \mathrm{L}$ with dextrose). Whole-cell configuration was obtained in voltage-clamp mode before proceeding to current-clamp. Cells with stable resting membrane potentials were used for data collection by PulseFit 8.74 (HEKA Electronics) software. Threshold was determined by the first action potential elicited by a series of depolarizing current injections (5 pA increments). Responses to sustained depolarization were assessed using 500 ms depolarization (25 pA increments for DRG or $10 \mathrm{pA}$ for SCG).

\section{Data analysis}

Electrophysiological data were analysed using PulseFit 8.74 (HEKA Electronics) and Origin 8.5.1 (Microcal), and were presented as means \pm standard error. Statistical significance was determined by unpaired Student's t-test or two proportions Z-test (comparison of proportion of cells producing resurgent currents). For multi-group statistical analysis, we used one-way ANOVA followed by Tukey post hoc test or Kruskal-Wallis followed by Dunn procedure depending on whether data showed a normal distribution. 


\section{Results}

\section{Clinical description}

Patients with variant c. $554 \mathrm{G}>\mathrm{A} ; \mathrm{p} . \mathrm{R} 185 \mathrm{H}$

Patient 1

A 54-year-old male was referred to the neurology outpatient clinic because of unpleasant paraesthesias in his feet and hands. The paraesthesias started in his feet at the age of 24 years and had a gradual progressive course. At age 52 years, the patient developed burning pain in his feet, especially in the morning. He also complained of 'electric current' in his soles and reddening of his feet during exercise. These complaints interfered with walking. There were no other autonomic symptoms. Short-term treatment with pregabalin and amitriptyline was ineffective. Similar complaints were reported in a brother. The patient's grandfather had difficulty in walking at advanced age. He was reported to have diminished heat sensation in his hands, and experienced painless burns.

Neurological examination was unremarkable with no signs of large fiber involvement. Extensive laboratory investigations and nerve conduction studies revealed no abnormalities, whereas quantitative sensory testing showed abnormal temperature thresholds for warmth and cold sensation. Skin biopsy showed markedly reduced intraepidermal nerve fiber density $(1.0 / \mathrm{mm})$, compared with age- and gender-specific normative values $(\geq 3.5 / \mathrm{mm})$. The patient was diagnosed as having small fiber neuropathy and DNA analysis showed a variant in $\mathrm{Na}_{\mathrm{v}} 1.7$ : c. $554 \mathrm{G}>\mathrm{A} ; \mathrm{p} . \mathrm{R} 185 \mathrm{H}$.

\section{Patient 2}

This 24-year-old female presented with severe pain in both feet. Her symptoms began at the age of 23 years with tingling in both feet, and gradually increased to involve the legs and hands, and became severe. Pain was described as maximal during rest, especially in the evening and during the nights, leading to a sleep disturbance. Drinking alcohol was reported to aggravate the pain. This patient reported only minor autonomic symptoms of occasional dizziness and dry mouth. Medical history was unremarkable, except for migraine since the age of 12 years. The family history was negative. Treatment with acetaminophen, antidepressants, anti-convulsants, mexiletine and opioids did not provide relief. Neurological examination demonstrated severe allodynia at the feet. There were no skin colour abnormalities. No signs of large fiber involvement were found. Extensive laboratory investigations showed no abnormalities. Nerve conduction studies were normal. Quantitative sensory testing showed abnormal temperature thresholds for warmth and cold sensation. Intraepidermal nerve fiber density was reduced $(4.9 / \mathrm{mm})$, compared to age- and gender-matched controls $(\geq 8.4 /$ 
$\mathrm{mm})$. The patient was diagnosed as having small fiber neuropathy and DNA analysis showed a variant in $\mathrm{Na}_{\mathrm{v}} 1.7$ : C.554G>A; p.R185 H.

Patients with variant $c .2215 A>G, p .1739 \mathrm{~V}$

Patient 3

This 51-year-old female, previously described in detail by Han et al. (2012), presented with a 37-year history of episodic burning pain, flushing and itching of the face, lower legs and feet, triggered by exercise or rising temperature, warm water or lying under a blanket and relieved by cooling. The patient had severe dysautonomic symptoms, which included dry mouth and eyes, blurred vision, orthostatic dizziness, alternating constipation and diarrhea, hyperhydrosis, heart rhythm palpitations and episodic swallowing difficulties. Treatment with amitriptyline did not provide relief. Physical examination demonstrated patchy allodynia at the feet and lower legs in a symmetrical pattern, and purple colored skin of the hands. Family history revealed similar symptoms in two sons and a sister, who did not consent to DNA analysis. DNA was obtained from an unaffected brother, and did not carry the $\mathrm{Na}_{\mathrm{v}} 1.7$ variant found in the patient.

Blood and chest $\mathrm{X}$-ray examinations were normal. Normal nerve conduction studies excluded large fiber involvement. Temperature thresholds were abnormal as measured by quantitative sensory testing. ${ }^{16} \mathrm{~A}$ skin biopsy revealed that intraepidermal nerve fiber density in the lower leg was $3.4 / \mathrm{mm}$, which was lower than the corresponding reported normative values $(4.3 / \mathrm{mm})$. The patient was diagnosed as having small fiber neuropathy, and DNA analysis showed a variant in $\mathrm{Na}_{\mathrm{v}}$. .7 : c.2215A>C; p.I739V.

\section{Patient 4}

This 66-year-old female with an unremarkable family history presented with sensory disturbances and severe autonomic dysfunction leading to disability. Her complaints began at the age of 64 years with numbness of the toes of the right foot. Six months later the numbness expanded to both feet and lower legs, making it difficult to maintain balance and the hands became affected. The patient experienced penetrating, stabbing pain, cramps and a feeling of 'electric shocks' in the feet, aggravated by exercise, walking or cold temperature. Resting or an electric heating blanket gave some relief. It became impossible to move without aids, due to excruciating pain.

The patient also suffers from severe dysautonomic symptoms (cardiac dysrhythmias, blood pressure fluctuations, diarrhea, urge incontinence, abdominal discomfort, hot flashes and hyperhidrosis). Because of arrhythmias during the night, the patient used continuous positive airway pressure. The patient is totally disabled and homebound due to these symptoms.

Carbamazepine, duloxetine and tramadol did not provide any relief, and were 
subsequently stopped. Pregabalin caused unacceptable side effects. Gabapentin, morphine and transcutaneous electrical nerve stimulation provided partial pain relief.

Physical examination showed allodynia and numbness in a stocking-glove distribution, with normal muscle strength and tendon reflexes. Laboratory tests, nerve conduction studies and a chest X-ray revealed no abnormalities. Quantitative sensory testing showed abnormal temperature thresholds for warmth and cold sensation. Skin biopsy showed an intraepidermal nerve fiber density of $2.3 / \mathrm{mm}$, which is reduced compared to normative values (fifth percentile: $3.2 / \mathrm{mm}$ ). The patient was diagnosed as having small fiber neuropathy, and DNA analysis showed a variant in $\mathrm{Na}_{\mathrm{v}}$ 1.7: c.2215A>C; p.I739V.

\section{Patient 5}

This 72-year-old female was referred to the neurological outpatient clinic because of a 5-year history of orthostatic intolerance, numbness and tingling of the feet and severe pain of the feet and ankles. Orthostatic dizziness, worst in the morning, interfered with getting out of bed or standing from a chair. She also complained of severe palpitations, for which the cardiologist could find no other explanation, and episodes of swelling and red discoloration of the feet that interfered with walking. She described the pain as burning, and noted that cooling of the feet or morphine, prescribed by a physician, tended to relieve it. Physical examination showed erythema and pitting edema of both feet and ankles. Light touch sense and vibration sense were abnormal up to the knees. Both Achilles tendon reflexes were absent. Nerve conduction studies revealed signs of a severe axonal sensorimotor polyneuropathy. Quantitative sensory testing showed abnormal temperature thresholds for cold sensation in the right foot, despite being incomplete due to severe pain during examination. Skin biopsy showed an intraepidermal nerve fiber density of $4.8 / \mathrm{mm}$, which is normal compared to normative values (fifth percentile: $2.2 / \mathrm{mm}$ ). The patient was diagnosed as having peripheral polyneuropathy with small and large fiber involvement, and DNA analysis revealed a variant in $\mathrm{Na}_{\mathrm{v}}$ 1.7: c.2215 A>C; p. $1739 \mathrm{~V}$.

\section{Molecular genetic analysis}

Sequence analysis of $S C N_{9} A$-coding exons from Patients 1 and 2 demonstrated a $\mathrm{G}$ to A substitution (c.554G>A) in both patients. This variant substitutes arginine 185 with histidine $(\mathrm{R} 185 \mathrm{H})$ in the linker between $\mathrm{DI} / \mathrm{S}_{2}$ and $\mathrm{DI} / \mathrm{S}_{3} . \mathrm{R} 185$ is highly conserved in all human voltage-gated sodium channels (Fig. 1A). The c.554G $>$ A substitution ( $r 573969684$ ) has been reported as a single nucleotide polymorphism with allele frequency of $0.6 \%$ (heterozygote frequency of $1.2 \%$ ) in the 1000 Genomes Project; and with $1.2 \%$ heterozygote frequency (55 
heterozygotes among 4700 individuals) reported in the Exome Variant Database (http://evs.gs.washington.edu/EVS). Screening a panel of 1000 ethnically matched (Dutch nationals of European ancestry) control population, we report the $\mathrm{c} .554 \mathrm{G}>\mathrm{A}$ in $0.4 \%$ of 1000 control subjects (0.4\% heterozygote frequency, $0.2 \%$ allele frequency).

As reported previously, ${ }^{16}$ sequencing of $S C N$ gA-coding exons from Patient 3 revealed the c.2215A>C, $\mathrm{Na}_{\mathrm{v}} 1.7 / 1739 \mathrm{~V}$ substitution. This substitution was also found in Patients 4 and 5 . The 1739 residue, located within the first transmembrane segment in domain II, is conserved in all human voltage-gated sodium channels (Fig. 1B), and in all mammalian $\mathrm{Na}_{\mathrm{v}}$ 1.7 orthologues reported to date. ${ }^{16}$

Sequencing of DNA from an asymptomatic brother of Patient 3 did not reveal any $\mathrm{SCN}_{9 A}$ variants. The C.2215A>C substitution in exon 13 of $S C N_{9} A$ has not been reported as a variant in the 1000 Genome Database (release 9 September 2011 ), but was reported with $0.5 \%$ frequency in the general population (25 heterozygotes among 4255 individuals) in the Exome Variant Database, and was found in $14(1.4 \%)$ of 1000 Dutch control subjects (all heterozygous; $0.7 \%$ of 2000 chromosomes).

Figure 1.

A

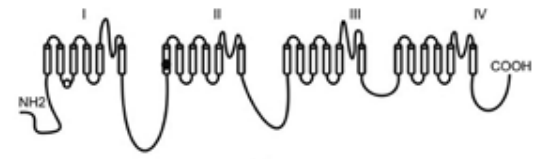

Na 1.1 FTFLRDPWNWLDF

$\mathrm{Na}_{\mathrm{y}}$ 1.2 FTFLRDPWNWLDF

$\mathrm{Na}_{\mathrm{v}} 1.3$ FTFLRDPWNWLDF

$\mathrm{Na}_{\mathrm{y}}$ 1.4 FTFLRDPWNWLDF

$\mathrm{Na}_{v} 1.5 \quad$ FTFLRDPWNWLDF

$\mathrm{Na}_{\mathrm{y}} 1.6 \quad$ FTFLRDPWNWLDF

Na,1.7 FTFLRDPWNWLDF

$\mathrm{Na}_{\mathrm{v}} 1.8$ FYFLRDPWNWLDF

$\mathrm{Na}_{4} 1.9$ FSFLRDPWNWLDS

$\mathrm{Na}_{\mathrm{y}} 1.7_{\mathrm{R} 185 \mathrm{H}}$ FTFLHDPWNWLDF

B

Na,1.1 VDLAITICIVLNT

$\mathrm{Na}_{\mathrm{v}} 1.2$ VDLAITICIVLNT

$\mathrm{Na}_{\mathrm{v}} 1.3$ VDLAITICIVLNT

$\mathrm{Na}_{\mathrm{v}} 1.4$ VDLGITICIVLNT

$\mathrm{Na}_{\mathrm{v}}$ 1.5 TDLTITMCIVLNT

$\mathrm{Na}_{\mathrm{v}} 1.6$ VDLAITICIVLNT

$\mathrm{Na}^{1} 1.7$ VDLAITICIVLNT

$\mathrm{Na}$ 1.8 AELTITLCIVVNT

$\mathrm{Na}_{\mathrm{v}} 1.9$ TELAITICIIINT

$\mathrm{Na}_{\mathrm{v}} 1.7_{1 \mathrm{r} 3 \mathrm{~g}}$ VDLAVTICIVLNT
Legend to figure 1. Schematic of a voltage-gated sodium channel showing the locations of the $\mathrm{R} 185 \mathrm{H}$ (open circle) and $1739 \mathrm{~V}$ (solid circle) substitutions and the aligned sequences for the linker between Nav1 DIS2 and DIS3 $(A)$ and for the relevant part of Nav1 DIIS1 (B). R185 (bold) and 1739 (bold) are both conserved in all known human voltage-gated sodium channels. 


\section{$\mathrm{R} 185 \mathrm{H}$ enhances resurgent currents and increases excitability in dorsal root ganglion neurons; $1739 \mathrm{~V}$ impairs slow-inactivation and increases excitability in ganglion neurons}

\section{Voltage-clamp analysis}

Figure $2 \mathrm{~A}$ shows representative $\mathrm{Na}_{\mathrm{v}} 1.7$ sodium currents recorded from DRG neurons (from $\mathrm{Na}_{\mathrm{v}} 1.8$ null mice) expressing wild-typechannels, and Fig. 2B from neurons expressing $\mathrm{R} 185 \mathrm{H}$ variant channels. Peak current densities were not significantly different (wild-type: $523 \pm 53 \mathrm{pA} / \mathrm{pF}, \mathrm{n}=48$; R185 H: $543 \pm 59$ $\mathrm{pA} / \mathrm{pF}, \mathrm{n}=46)$. As Fig. $2 \mathrm{C}$ shows, the voltage-dependence of channel activation was not significantly different between wild-type and $\mathrm{R} 185 \mathrm{H}$. The activation midpoints determined from fitting the data with a Boltzmann function were: $-20.8 \pm 1.0 \mathrm{mV}(\mathrm{n}=17)$ for wild-type and $-22.5 \pm 1.2 \mathrm{mV}(n=18)$ for $\mathrm{R} 185 \mathrm{H}$. The midpoints of fast-inactivation (from Boltzmann function fits of data) were not significantly different between wild-type $(-71.3 \pm 1.8 \mathrm{mV}, \mathrm{n}=15)$ and $\mathrm{R} 185 \mathrm{H}$ channels $(-72.1 \pm 1.4 \mathrm{mV}, \mathrm{n}=16)$ (Fig. 2D). Kinetics of deactivation were estimated from measurements of current decay at potentials from -100 to $-40 \mathrm{mV}$ after briefly activating the channels at $-20 \mathrm{mV}$ for $0.5 \mathrm{~ms}$. Figure $2 \mathrm{E}$ shows the rates of current decay for wild-type and $\mathrm{R}_{1} 85 \mathrm{H}$ variant channels. The deactivation rates of $\mathrm{R} 185 \mathrm{H}$ channels were not significantly different from those of wild-type channels across all voltages tested. The voltage-dependence of slow-inactivation (Fig. $2 \mathrm{~F}$ ) was not significantly different between $\mathrm{R} 185 \mathrm{H}$ and wild-type channels. When fitted with a Boltzmann function, the midpoints of slow-inactivation curves were $-54.5 \pm 2.1 \mathrm{mV}(\mathrm{n}=13)$ for wild-type and $-53.6 \pm$ $1.9 \mathrm{mV}(\mathrm{n}=14)$ for $\mathrm{R} 185 \mathrm{H}$ channels, and the component of non-inactivating channels measured at $10 \mathrm{mV}$ was not significantly different between wild-type and $\mathrm{R}_{1} 85 \mathrm{H}$ channels (wild-type: $15.6 \pm 1.9 \%, \mathrm{n}=13$; $\mathrm{R} 185 \mathrm{H}: 15.3 \pm 1.4 \%, \mathrm{n}=14$ ). To assess resurgent currents, DRG neurons were depolarized to $+30 \mathrm{mV}$ for 20 $\mathrm{ms}$ from a holding potential of -10omV followed by series of hyperpolarizations from omV to $-80 \mathrm{mV}$ for $100 \mathrm{~ms}$. Figure $2 \mathrm{G}$ and $\mathrm{H}$ show representative resurgent currents recorded from DRG neurons expressing wild-type or $\mathrm{R} 185 \mathrm{H}$ channels, respectively. As described previously, ${ }^{26}$ not all small DRG neurons produce resurgent current. In this study, we found that the percentage of DRG neurons that generate resurgent current was significantly higher for cells expressing $\mathrm{R} 185 \mathrm{H}$ (9 out 28 cells, $32 \%, \mathrm{P}<0.05$ ) than for $\mathrm{DRG}$ neurons expressing wild-type channels (3 out 29 cells, 10\%) (Fig. $2 \mathrm{H}$, inset). The amplitudes of resurgent current were $5.3 \% \pm 0.6 \%(n=3)$ and $6.6 \% \pm 1.3 \%(n=9)$ for DRG neurons expressing wild-type or $\mathrm{R} 185 \mathrm{H}$ channels, respectively.

As a comparator we assessed the $1739 \mathrm{~V}$ variant, which was identified in our previous series $^{8}$ in the patient with the most severe autonomic dysfunction and was subsequently found in two additional patients with small fiber neuropathy 
that included disabling autonomic symptoms (Patients 3, 4 and 5). As reported previously, ${ }^{16}$ the $1739 \mathrm{~V}$ variant impaired slow-inactivation, depolarizing the slowinactivation midpoint by $5.6 \mathrm{mV}$, and significantly increased the non-inactivating component at $10 \mathrm{mV}$ in DRG neurons. $1739 \mathrm{~V}$ did not alter activation, steady-state fast-inactivation, kinetics of deactivation or resurgent currents in DRG neurons.

Figure 2.

A

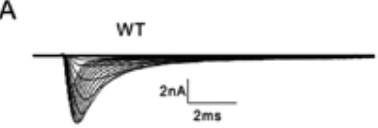

C

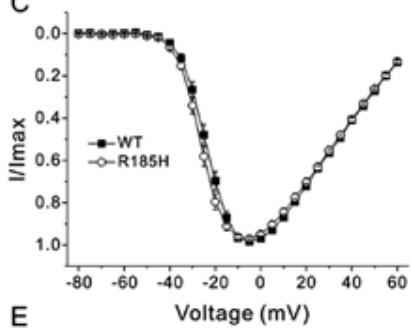

E

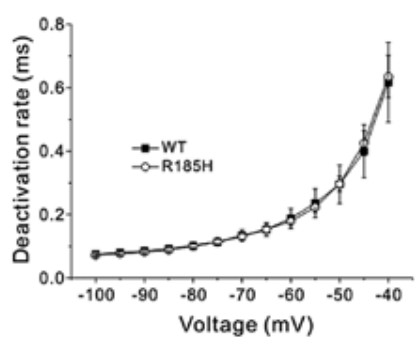

G

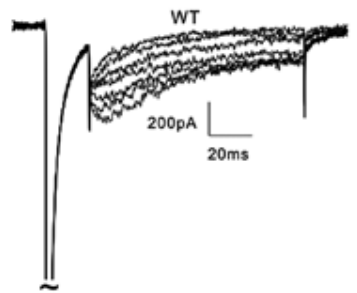

B
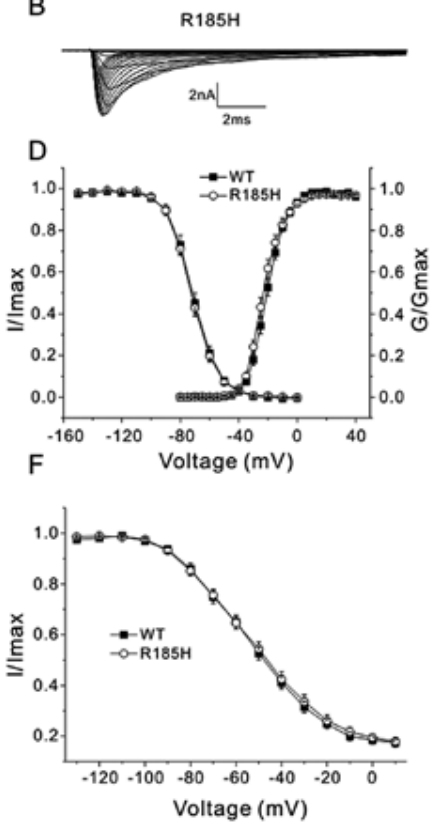

$\mathrm{H}$

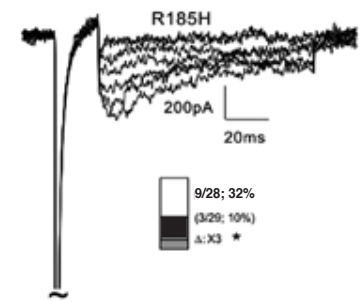

Chapter

6.

Legend to figure 2. Voltage-clamp analysis of wild-type (WT) and R $185 \mathrm{H}$ channels in DRG neurons. Representative current traces recorded from DRG neurons from $\mathrm{Na}_{v} 1.8$-null mice expressing wildtype $(A)$ or $\mathrm{R} 185 \mathrm{H}(\mathrm{B})$, evoked by voltage steps $(100 \mathrm{~ms})$ from -80 to $60 \mathrm{mV}$ in 5 - $\mathrm{mV}$ increments, from a holding potential of $-100 \mathrm{mV}$. (C) Normalized peak current-voltage relationship curves for wild-type and $\mathrm{R} 185 \mathrm{H}$ channels. (D) Comparison of voltage-dependent activation and steadystate fast inactivation for wild-type and $\mathrm{R} 185 \mathrm{H}$ channels. $\mathrm{R} 185 \mathrm{H}$ does not alter activation or fast-inactivation. (E) Comparison of time constants for deactivation. $\mathrm{R} 185 \mathrm{H}$ displays similar deactivation rates as wild-type channels. (F) Comparison of steady-state slow-inactivation curves between wild-type and $\mathrm{R} 185 \mathrm{H}$ variant channels. $\mathrm{R} 185 \mathrm{H}$ does not alter steady-state slowinactivation. ( $\mathrm{G}$ and $\mathrm{H}$ ) Representative resurgent current traces recorded from DRG neurons expressing wild-type $(\mathrm{G})$ or $\mathrm{R} 185 \mathrm{H}(\mathrm{H})$ channels. Inset bar graph showing a higher percentage of DRG neurons expressing $\mathrm{R} 185 \mathrm{H}$ (9 of 28 cells, $32 \%$ ) (black) compared to cells expressing wildtype channels ( 3 of 29 cells, $10 \%$ ) (grey) produce resurgent currents. $* \mathrm{P}<0.05$. 


\section{Current-clamp analysis: both $\mathrm{R} 185 \mathrm{H}$ and $\mathrm{I} 739 \mathrm{~V}$ render dorsal root ganglion neurons hyperexcitable}

Patients 1 and 2, who carried the R185 $\mathrm{H}$ variant, experienced pain, displayed abnormal temperature thresholds and exhibited reduced intraepidermal nerve fiber density, all symptoms of DRG neuron dysfunction. We therefore assessed the effect of the $\mathrm{R} 185 \mathrm{H}$ channels on DRG neuron excitability, using current-clamp recording after expressing wild-type or $\mathrm{R} 185 \mathrm{H}$ variant channels in small DRG neurons from rat pups. Patients 3,4 and 5 , who carried the ${ }^{7} 739 \mathrm{~V}$ variant, also experienced pain, displayed abnormal temperature thresholds and exhibited reduced intraepidermal nerve fiber density. Therefore, $1739 \mathrm{~V}$ channels were also transfected into DRG neurons (o-5 days old) as a comparator for assessment by current-clamp recording (Fig. 3). Input resistance was not significantly different among DRG neurons expressing wild-type channels $(1.0 \pm 0.1 \mathrm{G} \Omega, \mathrm{n}=$ 35), $\mathrm{R} 185 \mathrm{H}$ variant channels $(1.1 \pm 0.1 \mathrm{G} \Omega, \mathrm{n}=38)$ and $1739 \mathrm{~V}$ variant channels (1.1 $\pm 0.1 \mathrm{G} \Omega, \mathrm{n}=30$ ). The resting membrane potential of DRG neurons expressing $\mathrm{R} 185 \mathrm{H}$ variant channels $(-55.3 \pm 0.7 \mathrm{mV}, \mathrm{n}=38)$ was not significantly different from that of DRG neurons expressing wild-type channels $(-56.9 \pm 0.8 \mathrm{mV}, \mathrm{n}=$ 35), whereas $1739 \mathrm{~V}$ variant channels depolarized resting membrane potential of DRG neurons by $4.7 \mathrm{mV}(-52.2 \pm 0.6 \mathrm{mV}, \mathrm{n}=30, \mathrm{P}<0.001)$ (Fig. 3D).

To assess the effect of variant channels on current threshold of DRG neurons, we injected series of depolarization currents to determine the current threshold for producing the first all-or-none action potential. DRG neurons expressing $\mathrm{R} 185 \mathrm{H}$ and DRG neurons expressing $1739 \mathrm{~V}$ variant channels both displayed significantly reduced current threshold compared to DRG neurons expressing wild-type channels. Figure $3 \mathrm{~A}$ shows traces from a representative DRG neuron expressing wild-type channels. For this neuron, the current threshold to generate first action potential was $100 \mathrm{pA}$, and a $95 \mathrm{pA}$ current injection only evoked a small, graded membrane potential depolarization. Figure $3 B$ shows recordings from a representative DRG neuron expressing $\mathrm{R} 185 \mathrm{H}$ channels, in which a lower current injection of $65 \mathrm{pA}$ could produce an overshoot action potential. Figure ${ }_{3} \mathrm{C}$ shows recordings from a representative DRG neuron expressing $1739 \mathrm{~V}$ channels, in which a $55 \mathrm{pA}$ current input could elicit an action potential. As indicated in Fig. $3 \mathrm{E}$, average current threshold was significantly decreased for DRG neurons expressing R $185 \mathrm{H}$ variant channels $(63 \pm 7 \mathrm{pA}, \mathrm{n}=38, \mathrm{P}<0.001)$ and $1739 \mathrm{~V}$ variant channels $(61 \pm 7 \mathrm{pA}, \mathrm{n}=30, \mathrm{P}<0.001)$ compared with $\mathrm{DRC}$ neurons expressing wild-type channels $(111 \pm 11 \mathrm{pA}, \mathrm{n}=35)$. In contrast, action potential amplitude was not significantly different among DRG neurons expressing wild-type $(114.8 \pm 1.6 \mathrm{mV}, \mathrm{n}=35), \mathrm{R} 185 \mathrm{H}(114.8 \pm 1.5 \mathrm{mV}, \mathrm{n}=38)$ and $1739 \mathrm{~V}$ channels (111.7 $\pm 1.8 \mathrm{mV}, \mathrm{n}=30$ ) (Fig. $3 \mathrm{~F}$ ).

We next investigated the responses of DRG neurons expressing wild-type, $R 185 \mathrm{H}$ and $1739 \mathrm{~V}$ channels to series of $500 \mathrm{~ms}$ sustained depolarizations. Compared 
to DRG neurons expressing wild-type channels, both DRG neurons expressing $\mathrm{R} 185 \mathrm{H}$ channels and DRG neurons expressing $1739 \mathrm{~V}$ channels generated more action potentials in response to sustained depolarizing stimuli. Figure $4 \mathrm{~A}$ shows the responses from three representative neurons which expressed wild-type, $\mathrm{R} 185 \mathrm{H}$ or $1739 \mathrm{~V}$ channels, respectively, evoked by 500 ms current steps at one, two and three times the current threshold. DRG neurons expressing wild-type channels produced only two or three action potentials in response to current inputs at two or three times the current threshold, whereas DRG neurons expressing $\mathrm{R} 185 \mathrm{H}$ channels and DRG neurons expressing $1739 \mathrm{~V}$ channels both produced more action potentials. As summarized in Fig. 4B, DRG neurons expressing $\mathrm{R} 185 \mathrm{H}$ and $1739 \mathrm{~V}$ channels generated significantly more action potentials, compared with DRG neurons expressing wild-type channels, at all stimulus intensities $>75 \mathrm{pA}$.

Figure 3.
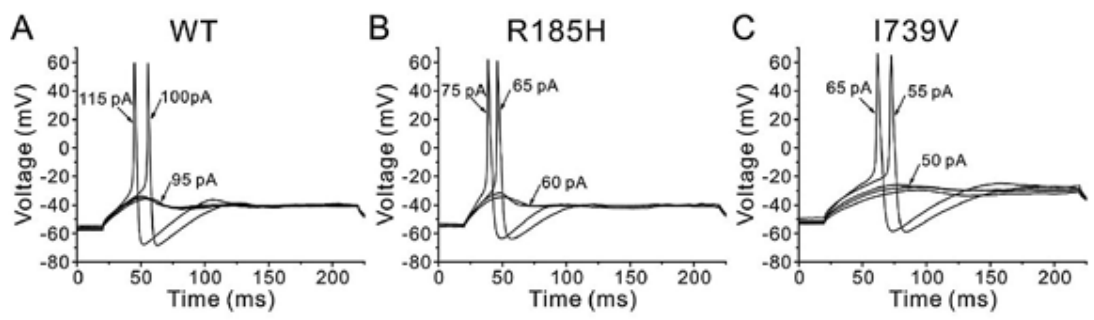

D

$\mathrm{E}$

$\mathrm{F}$
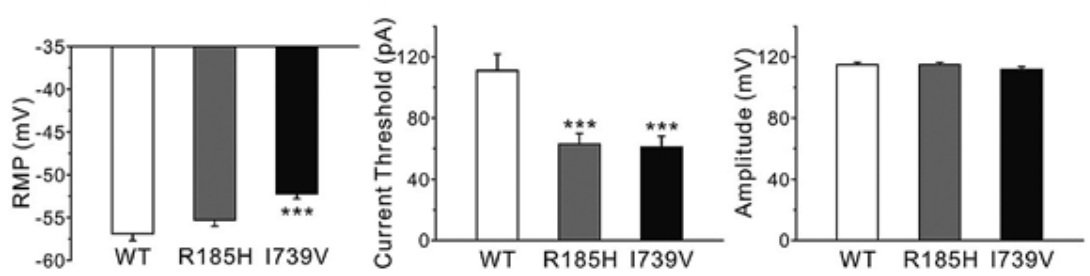

Legend to figure 3. R185 H and I739V both render DRG neurons hyperexcitable. (A) Representative traces from a DRG neuron (0 - to 5-day-old rat) expressing wild-type (WT) channels, showing sub-threshold response to $95 \mathrm{pA}$ current injection and subsequent action potentials evoked by injections of $100 \mathrm{pA}$ (current threshold for this neuron) and $115 \mathrm{pA}$. (B) Representative traces from a DRG neuron expressing $\mathrm{R} 185 \mathrm{H}$ variant channels, showing a lower current threshold $(65$ pA for this neuron) for action potential generation. (C) Representative traces from a DRG neuron expressing $1739 \mathrm{~V}$ variant channels, showing a lower current threshold (55 pA for this neuron) for action potential generation. (D) $\mathrm{R} 185 \mathrm{H}$ does not alter resting membrane potential (RMP) of DRG neurons, but $1739 \mathrm{~V}$ significantly depolarizes RMP by $4.7 \mathrm{mV}$. $* * * * \mathrm{P}<0.001$ versus wild-type. (E) Comparison of current threshold among DRG neurons expressing wild-type, $\mathrm{R} 185 \mathrm{H}$ or $1739 \mathrm{~V}$ channels. Current thresholds of DRG neurons are significantly reduced after expression $\mathrm{R} 185 \mathrm{H}$ or $1739 \mathrm{~V}$ variant channels. $* * \cdots \mathrm{P}<0.001$ versus wild-type. (F) Comparison of action potential amplitude among DRG neurons expressing wild-type, $\mathrm{R} 185 \mathrm{H}$ and $1739 \mathrm{~V}$ channels. $\mathrm{R} 185 \mathrm{H}$ and $1739 \mathrm{~V}$ do not have an effect on action potential amplitude of DRG neurons. 
Figure 4.

A

WT

$\mathrm{R} 185 \mathrm{H}$

$1739 \mathrm{~V}$
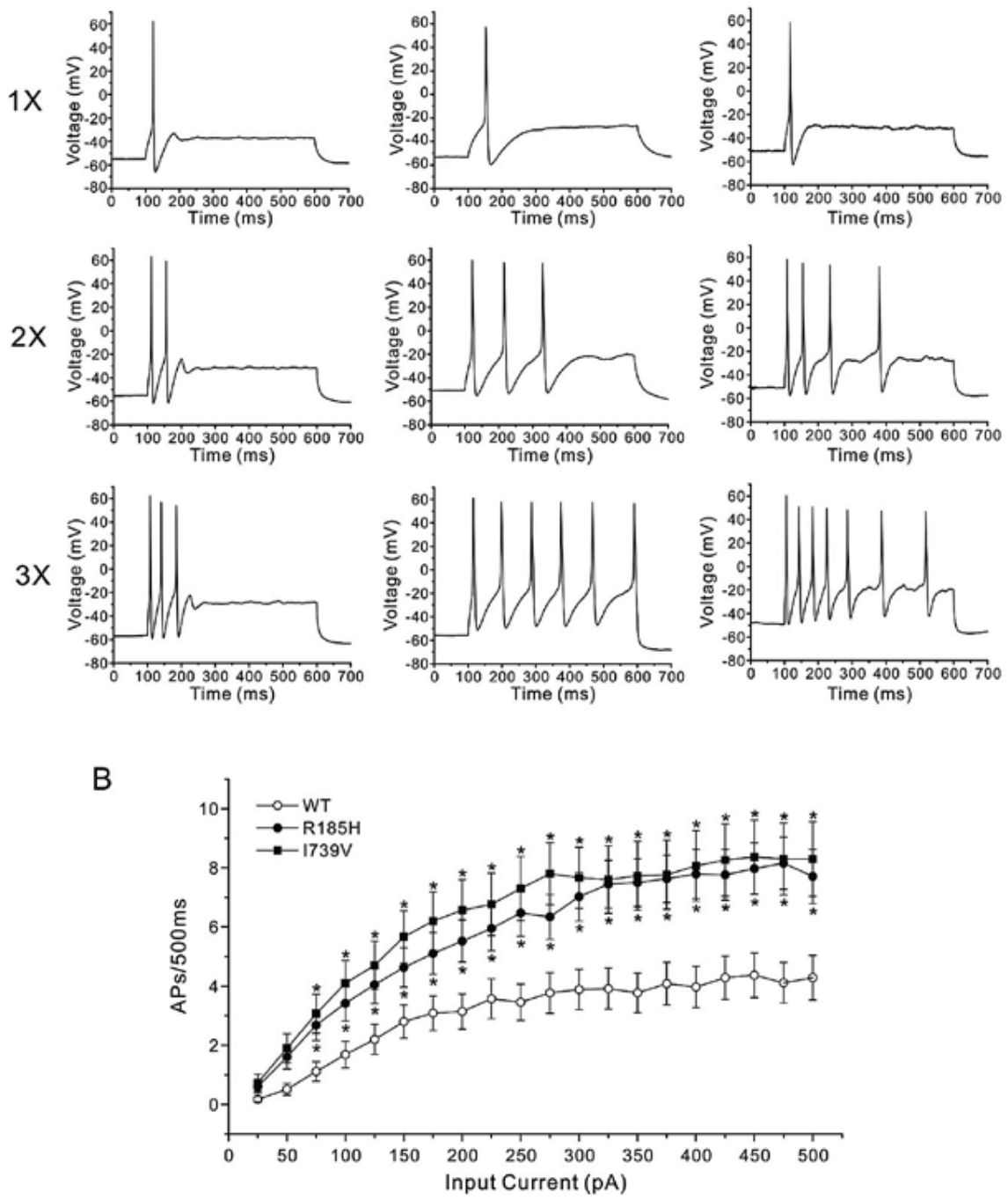

Legend to figure 4. $\mathrm{R} 185 \mathrm{H}$ and $1739 \mathrm{~V}$ both increase repetitive firing in DRG neurons. (A) Representative responses of DRG neurons ( 0 - to 5-day-old rat) expressing wild-type (WT), $\mathrm{R} 185 \mathrm{H}$ and $1739 \mathrm{~V}$ channels, respectively, to $500 \mathrm{~ms}$ depolarizing current steps that are one, two and three times (top, middle and bottom traces, respectively) the current threshold for action potential generation. (B) Comparison of mean action potential (AP) spike numbers among DRC neurons expressing wild-type, $\mathrm{R} 185 \mathrm{H}$ and $1739 \mathrm{~V}$ channels across a range of $500 \mathrm{~ms}$ step current injections from 25 to $500 \mathrm{pA}$. $* \mathrm{P}<0.05$ versus wild type. $\mathrm{R} 185 \mathrm{H}$ and $\mathrm{I} 739 \mathrm{~V}$ variant channels both increase spike numbers compared to wild-type. 


\section{$\mathrm{R} 185 \mathrm{H}$ does not alter biophysical properties of $\mathrm{Na}_{\mathrm{v}} 1.7$, while $1739 \mathrm{~V}$ impairs slow-inactivation in superior cervical ganglion neurons}

Sodium channel $\mathrm{Na}_{\mathrm{v}} 1.7$ is known to be expressed in both DRG and SCC neurons. ${ }^{9}, 10$ The two patients carrying the $\mathrm{R} 185 \mathrm{H}$ variant displayed minimal autonomic symptoms. In contrast, Patients 3,4 and 5 , who carried the $1739 \mathrm{~V}$ variant, displayed severe autonomic dysfunction. We therefore asked whether the variant $\mathrm{R} 185 \mathrm{H}$ or $1739 \mathrm{~V}$ channels change the biophysical properties of $\mathrm{Na}_{\mathrm{v}} \mathrm{T} .7$ within sympathetic ganglion neurons. To address these questions we transfected wild-type, $\mathrm{R} 185 \mathrm{H}$ or $1739 \mathrm{~V}$ channels into SCG neurons of rat pups by biolistic transfections and performed voltage-clamp analysis.

Figure $5 \mathrm{~A}-\mathrm{C}$ shows representative $\mathrm{Na}_{\mathrm{v}} 1.7$ sodium currents recorded from small SCG neurons (diameter $\leq 25 \mu \mathrm{m}$ ) expressing wild-type channels, $\mathrm{R} 185 \mathrm{H}$ variant, or $1739 \mathrm{~V}$ variant channels, respectively. Peak current densities were not significantly different among cells transfected with wild-type $(708 \pm 115 \mathrm{pA} / \mathrm{pF}$, $\mathrm{n}=36), \mathrm{R} 185 \mathrm{H}(694 \pm 110 \mathrm{pA} / \mathrm{pF}, \mathrm{n}=26)$ and $1739 \mathrm{~V}$ channels $(650 \pm 90 \mathrm{pA} / \mathrm{pF}$, $n=34)$. As the $I-V$ curves in Fig. $5 D$ demonstrate, the voltage-dependence of activation was not significantly different among wild-type, $\mathrm{R} 185 \mathrm{H}$ and $1739 \mathrm{~V}$. The activation midpoints determined from fitting the data with a Boltzmann function were $-23.7 \pm 1.6 \mathrm{mV}(n=22)$ for wild-type, $-22.0 \pm 1.6 \mathrm{mV}(n=14)$ for $\mathrm{R} 185 \mathrm{H}$ and $-23.2 \pm 1.9 \mathrm{mV}(\mathrm{n}=18)$ for $1739 \mathrm{~V}$. The midpoints of fast-inactivation (from Boltzmann function fits of data) were not significantly different among wildtype $(-75.2 \pm 1.3 \mathrm{mV}, \mathrm{n}=22), \mathrm{R} 185 \mathrm{H}(-72.8 \pm 1.4 \mathrm{mV}, \mathrm{n}=14)$ and $1739 \mathrm{~V}$ channels $(-74.1 \pm 1.6 \mathrm{mV}, \mathrm{n}=18)$ (Fig. $5 \mathrm{E})$. The rates of current decay for wild-type, $\mathrm{R} 185 \mathrm{H}$ and $1739 \mathrm{~V}$ variant channels, as shown in Fig. $5 \mathrm{~F}$, were not significantly different across all voltages tested.

Slow-inactivation was impaired in $1739 \mathrm{~V}$, but not $\mathrm{R} 185 \mathrm{H}$ channels within the SCG cell background. The voltage-dependence of slow-inactivation (Fig. ${ }_{5} \mathrm{G}$ ) was not significantly different between $\mathrm{R} 185 \mathrm{H}$ and wild-type channels within SCG neurons. The midpoints of slow-inactivation were $-57.8 \pm 1.3 \mathrm{mV}(n=13)$ for wild-type and $-54.6 \pm 1.7 \mathrm{mV}(n=13)$ for $\mathrm{R} 185 \mathrm{H}$ channels. In contrast, the voltagedependence of slow-inactivation was significantly impaired in $1739 \mathrm{~V}$ channels compared with wild-type channels, and the midpoint of slow-inactivation (from Boltzmann function fits of data) was $-51.7 \pm 1.9 \mathrm{mV}(\mathrm{n}=12, \mathrm{P}<0.05)$ for $1739 \mathrm{~V}$ channels, $-6 \mathrm{mV}$ shifted in the depolarizing direction. The component of noninactivating channels measured at $10 \mathrm{mV}$ was not significantly different between wild-type and $\mathrm{R} 185 \mathrm{H}$ channels (wild-type: $10.4 \pm 1.3 \%, \mathrm{n}=13$; $\mathrm{R} 185 \mathrm{H}: 11.1 \pm$ $1.5 \%, n=13$ ), whereas for $1739 \mathrm{~V}$ channels the non-inactivated component was significantly increased (17.4 $\pm 1.4 \%, n=12, \mathrm{P}<0.01$ ) (Fig. 5G). Resurgent current protocols did not evoke resurgent currents in SCG neurons transfected with either wild-type, $\mathrm{R} 185 \mathrm{H}$ or $1739 \mathrm{~V}$ variant channels (Fig. $5 \mathrm{H}-J$ ). 
Figure 5.
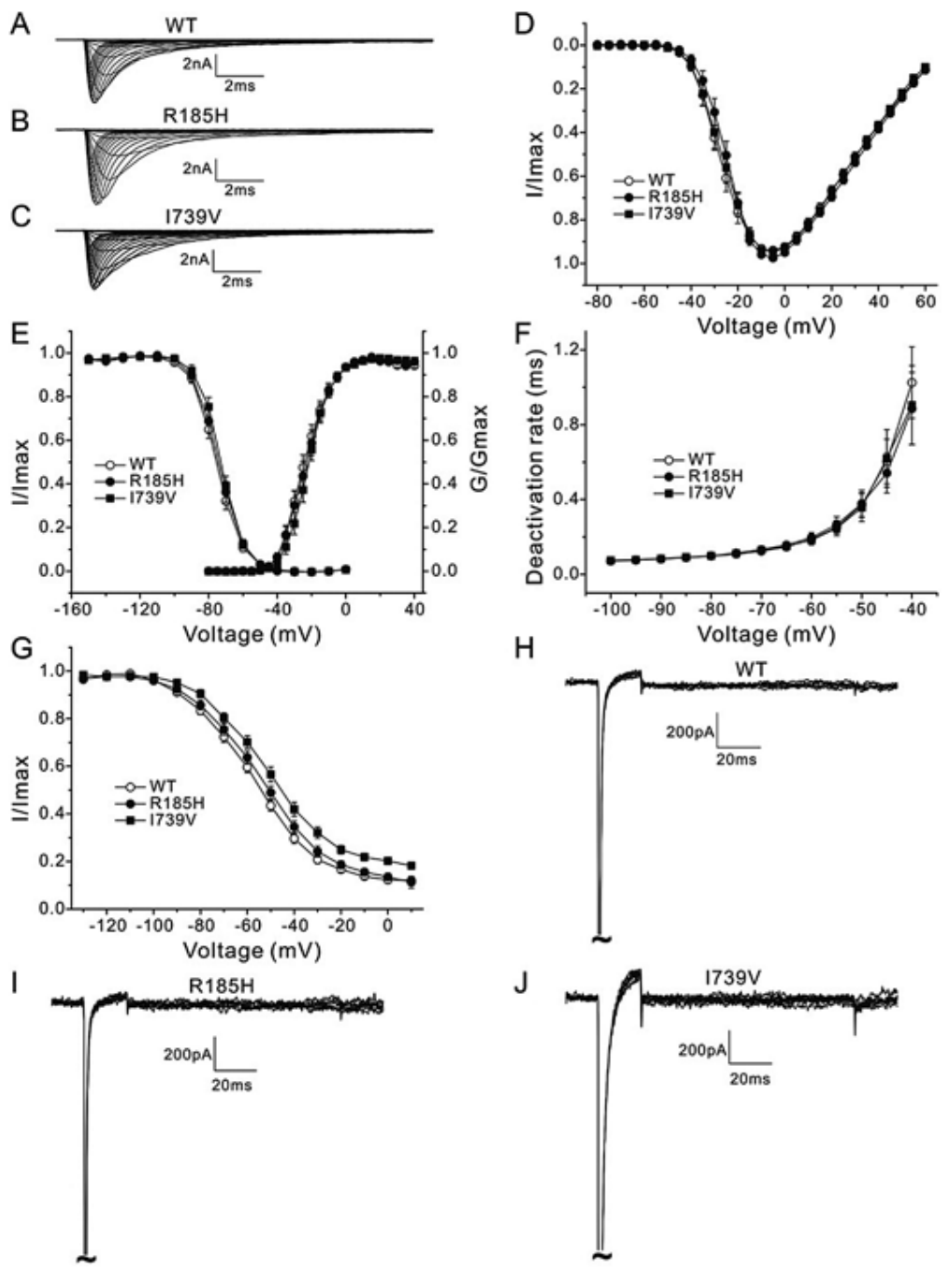

Legend to figure 5. Voltage-clamp analysis of wild-type (WT), R $185 \mathrm{H}$ and $1739 \mathrm{~V}$ channels in SCG neurons. Representative current traces recorded from SCG neurons ( 0 - to 5 -day-old rat) expressing wild-type (A), R185H (B) or $1739 \mathrm{~V}$ channels (C), evoked by voltage steps (100 ms) from $-80 \mathrm{mV}$ to $60 \mathrm{mV}$ in $5-\mathrm{mV}$ increments, from a holding potential of $-100 \mathrm{mV}$. (D) Normalized peak current-voltage relationship curves for wild-type, $\mathrm{R} 185 \mathrm{H}$ and $\mathrm{I} 739 \mathrm{~V}$ channels. (E) Comparison of voltage dependent activation and steady-state fast-inactivation for wild-type, $\mathrm{R} 185 \mathrm{H}$ and ${ }_{1739} \mathrm{~V}$ channels. $\mathrm{R} 185 \mathrm{H}$ and $1739 \mathrm{~V}$ do not alter activation or fast-inactivation. (F) Comparison of time constants for deactivation. $\mathrm{R} 185 \mathrm{H}$ and $1739 \mathrm{~V}$ both display similar deactivation rates as wild-type channels. (G) Comparison of steady-state slowinactivation curves among wild type, $\mathrm{R} 185 \mathrm{H}$ and $1739 \mathrm{~V}$ variant channels. $\mathrm{R} 185 \mathrm{H}$ does not alter steady-state slow-inactivation. $1739 \mathrm{~V}$ shifts the steady-state slow-inactivation to the right by $6.1 \mathrm{mV}$ and increases the non-inactivated current at $10 \mathrm{mV}$. (H-J) SCG neurons expressing wild-type $(\mathrm{H}), \mathrm{R} 185 \mathrm{H}(\mathrm{I})$ or $1739 \mathrm{~V}(\mathrm{~J})$ channels do not produce resurgent currents. 


\section{$\mathrm{R} 185 \mathrm{H}$ does not alter excitability of superior cervical ganglion neurons whereas $1739 \mathrm{~V}$ renders superior cervical ganglion neurons hypoexcitable}

To assess the effect of the $\mathrm{R} 185 \mathrm{H}$ and $1739 \mathrm{~V}$ variant channels on SCG neuron excitability, we performed current-clamp recording after expressing wild-type, $\mathrm{R} 185 \mathrm{H}$ and $1739 \mathrm{~V}$ variant channels in SCG neurons from rat pups. We assessed cells displaying strong green fluorescence, which provided a marker for transfected cells, ${ }^{24}$ and confirmed the presence of the variant channels within SCG neurons by voltage-clamp.

Input resistance was not significantly different among SCG neurons expressing wild-type $(1.2 \pm 0.1 \mathrm{G} \Omega, \mathrm{n}=28), \mathrm{R} 185 \mathrm{H}(1.1 \pm 0.1 \mathrm{G} \Omega, \mathrm{n}=18)$ and $1739 \mathrm{~V}$ channels $(1.0 \pm 0.1 \mathrm{G} \Omega, \mathrm{n}=23)$. The resting membrane potential of SCG neurons expressing $\mathrm{R} 185 \mathrm{H}$ variant channels $(-52.1 \pm 0.9 \mathrm{mV}, \mathrm{n}=18)$ was not significantly different from that of SCG neurons expressing wild-type channels $(-50.8 \pm 0.6$ $\mathrm{mV}, \mathrm{n}=28)$. In contrast, $1739 \mathrm{~V}$ channels produced a significant depolarizing shift of $4 \mathrm{mV}$ in resting membrane potential of SCG neurons $(-46.8 \pm 0.8 \mathrm{mV}, \mathrm{n}$ $=23, \mathrm{P}<0.001$ ) (Fig. 6D).

While, as described earlier, the $\mathrm{R} 185 \mathrm{H}$ and $1739 \mathrm{~V}$ variants both reduced the current threshold in DRG neurons, the effects of these two variants on the current threshold of SCG neurons were dramatically different. Figure 6A show traces recorded from a representative SCG neuron transfected with wild-type channels. In contrast to the high threshold of DRG neurons expressing wildtype channels, SCG neurons expressing wild-type channels displayed a much lower current threshold; for this SCG neuron, 15 pA current injection evoked the first all-or-none action potential. As shown in Fig. 6B, recordings from a representative SCG neuron that expressed $\mathrm{R} 185 \mathrm{H}$ channels showed that the same depolarizing current stimulus (15 pA) could evoke an all-or-none action potential. Figure $6 \mathrm{C}$ shows typical traces recorded from a SCG neuron expressing $1739 \mathrm{~V}$ channels, where a larger current injection of $30 \mathrm{pA}$ was required to produce the first overshooting action potential. As shown in Fig. $6 \mathrm{E}$, the average current threshold of SCG neurons expressing $\mathrm{R} 185 \mathrm{H}$ variant channels $(18.6 \pm 2.4 \mathrm{pA}, \mathrm{n}$ $=18$ ) was not significantly different from that of SCG neurons expressing wildtype channels $(17.9 \pm 1.5 \mathrm{pA}, \mathrm{n}=28)$. In contrast, $1739 \mathrm{~V}$ channels $(27.8 \pm 2.8 \mathrm{pA}$, $\mathrm{n}=23, \mathrm{P}<0.01$ ) significantly increased the current threshold of SCG neurons. The effects of $\mathrm{R} 185 \mathrm{H}$ and $1739 \mathrm{~V}$ channels on the action potential amplitude of SCG weremarkedly different. As shown in Fig. $6 \mathrm{~F}$, the action potential amplitude of SCG neurons expressing R185H channels $(100.5 \pm 3.8 \mathrm{pA}, \mathrm{n}=18)$ was not significantly different from that of SCG neurons expressing wild-type channels ( $98.2 \pm 2.6 \mathrm{pA}, \mathrm{n}=28)$. In contrast, the action potential amplitude of SCC neurons $(81.0 \pm 3.8 \mathrm{pA}, \mathrm{n}=23, \mathrm{P}<0.001)$ was significantly reduced following expression of $1739 \mathrm{~V}$ channels. 
Figure 6.
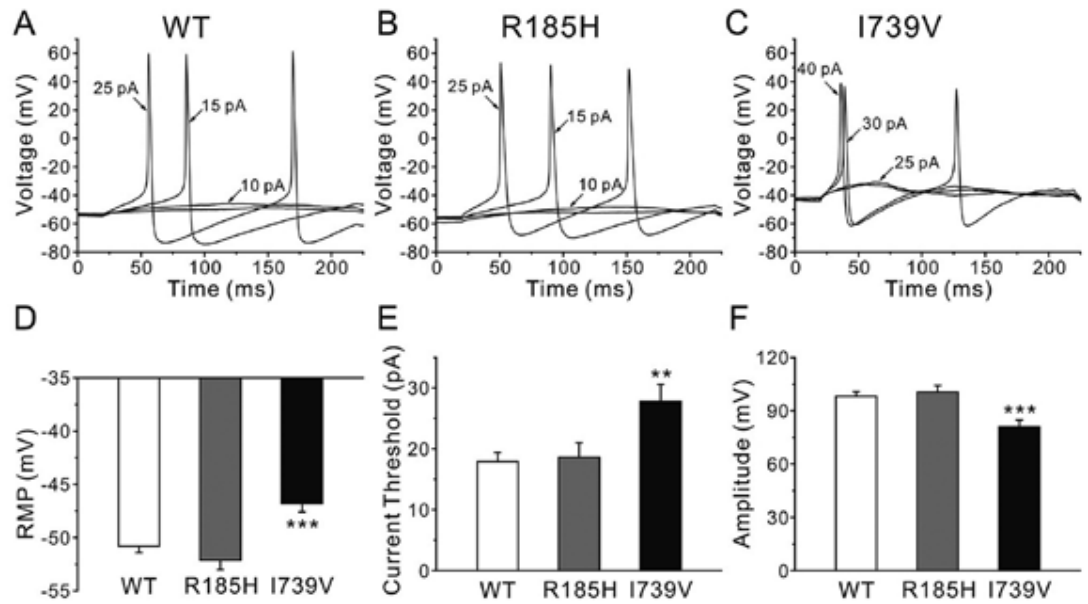

Legend to figure 6. $\mathrm{R} 185 \mathrm{H}$ does not alter SCG neurons excitability, but I739V renders SCG neurons (0 - to 5-day-old rat) hypoexcitable. (A) Representative traces from a SCG neuron expressing wildtype (WT) channels show sub-threshold responses to $10 \mathrm{pA}$ current injection and subsequent all-or-none action potentials elicited by current inputs of $15 \mathrm{pA}$ and $25 \mathrm{pA}$. (B) Representative traces from a SCG neuron expressing $\mathrm{R} 185 \mathrm{H}$ channels, showing similar current threshold for action potential generation as SCG neuron expressing wild-type channels. (C) Representative traces from a SCG neuron expressing $1739 \mathrm{~V}$ channels, showing that a higher current injection of $30 \mathrm{pA}$ is required to generate the first all-or-none action potential. (D) $\mathrm{R} 185 \mathrm{H}$ does not alter the RMP of SCG neurons, but $1739 \mathrm{~V}$ causes a significantly depolarizating shift in RMP by 4.0 $\mathrm{mV}$. $* * * \mathrm{P}<0.001$ versus wild-type. (E) Average current threshold of SCG neurons expressing $\mathrm{R} 185 \mathrm{H}$ channels is not significantly different from SCG neurons expressing wild-type channels, whereas it is significantly increased after expression of $1739 \mathrm{~V}$ variant channels. $* * \mathrm{P}<0.01$ versus wild-type. (F) SCG neurons expressing R $185 \mathrm{H}$ channels display similar action potential amplitude as neurons expressing wild-type channels, whereas the amplitude is significantly smaller after expression $1739 \mathrm{~V}$ channels. $* * * \mathrm{P}<0.001$ versus wild-type.

As with DRG neurons, we also investigated the responses of SCG neurons to series of 500 ms sustained depolarizations after expressing wild-type, $\mathrm{R}_{1} 8{ }_{5} \mathrm{H}$ and $1739 \mathrm{~V}$ channels. Because current threshold was lower in SCG neurons, the span of injected currents was narrowed to 10-200 pA for these cells. Figure $7 \mathrm{~A}$ shows responses from three representative SCG neurons which expressed wild type, $\mathrm{R} 185 \mathrm{H}$ or $1739 \mathrm{~V}$ channels, respectively, to $500 \mathrm{~ms}$ current steps at one, two and three times the current threshold. SCG neurons expressing $\mathrm{R}_{1} 85 \mathrm{H}$ channels displayed a pattern of repetitive action potentials similar to that in SCG neurons expressing wild-type channels and, in response to stimuli at three times the threshold, produced repetitive firing (seven action potentials in $500 \mathrm{~ms}$ ) that was sustained throughout the $500 \mathrm{~ms}$ depolarizing stimulus. In contrast, SCG neurons expressing $1739 \mathrm{~V}$ channels generated fewer action potentials, and 
could not sustain firing throughout the 500 ms stimulus. Figure $7 \mathrm{~B}$ summarizes the different effects of the $\mathrm{R} 185 \mathrm{H}$ and $1739 \mathrm{~V}$ variants on the response of SCG neurons to prolonged stimuli, and shows that in SCG neurons, the $\mathrm{R} 185 \mathrm{H}$ variant does not affect repetitive firing, whereas the $1739 \mathrm{~V}$ variant attenuates it. Taken together, these results show that $\mathrm{R} 185 \mathrm{H}$ has no effects on the excitability of SCG neurons, while in contrast, $1739 \mathrm{~V}$ renders SCG neurons hypoexcitable.

Figure 7.

A

WT
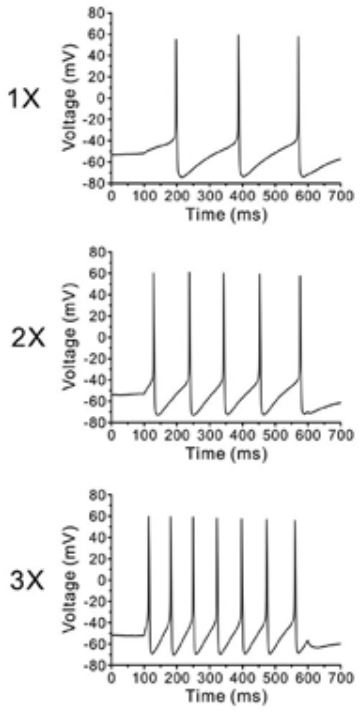

$\mathrm{R} 185 \mathrm{H}$
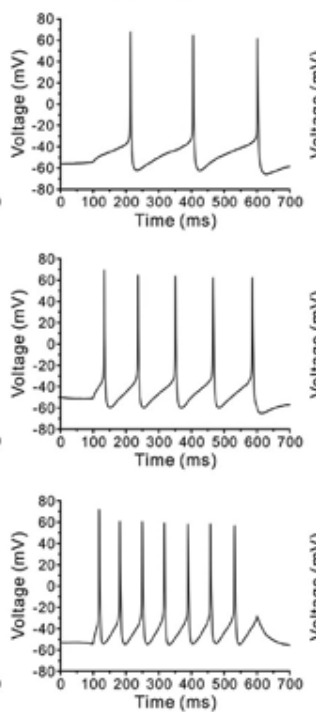

$1739 \mathrm{~V}$
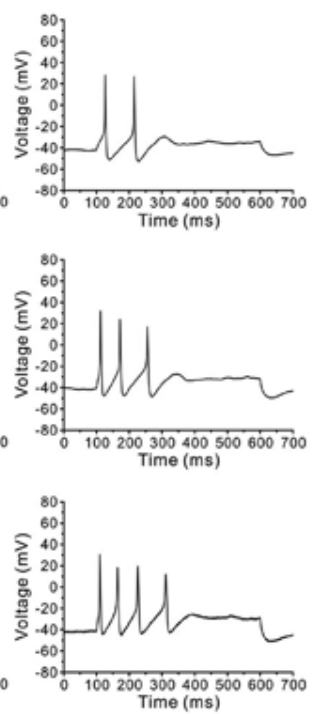

B

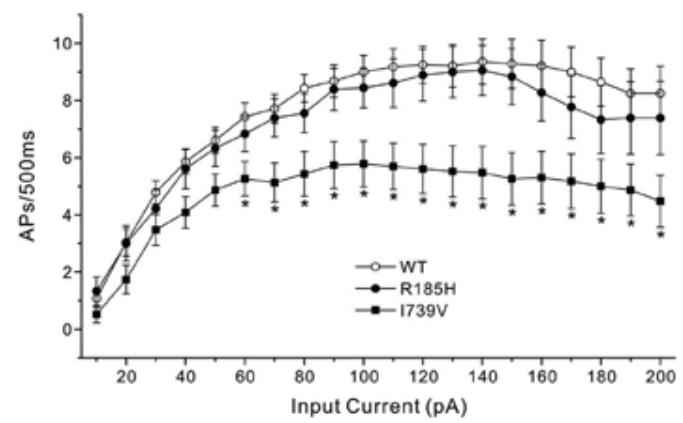

Legend to figure 7. $1739 \mathrm{~V}$ attenuates repetitive firing in SCG neurons, while $\mathrm{R} 185 \mathrm{H}$ does not affect repetitive firing. (A) Representative responses of SCG neurons (0 - to 5-day-old rat) expressing wild-type (WT), R185 H and $1739 \mathrm{~V}$ channels, respectively, to $500 \mathrm{~ms}$ depolarizing current steps that are one, two and three times (top, middle and bottom traces, respectively) the current threshold for action potential (AP) generation. (B) Comparison of mean action potential spike numbers between SCG neurons expressing wild-type, $\mathrm{R} 185 \mathrm{H}$ and $1739 \mathrm{~V}$ channels across a range of 500 ms step current injections from 10 to 200 pA. Compared to wild-type, $1739 \mathrm{~V}$ attenuates spike numbers, while $\mathrm{R} 185 \mathrm{H}$ does not. $* \mathrm{P}<0.05$ versus wild-type. 


\section{Discussion}

While small fiber neuropathy usually presents a clinical picture of pain, sensory loss and autonomic symptoms, occasionally patients display pain and sensory loss with only minimal autonomic dysfunction. We have reported the presence of functional $\mathrm{Na}_{\mathrm{v}} 1.7$ variants in 8 of a series of 28 patients with biopsy-confirmed small fiber neuropathy, with all except two of these patients manifesting autonomic dysfunction. ${ }^{8}$ One of the patients in this series (Patient 3 in this study) with the most severe autonomic symptoms, and two additional patients reported here (Patients 4 and 5) with severe autonomic dysfunction as well as limb pain, carry the single amino acid substitution $1739 \mathrm{~V}$. In contrast, two unrelated patients with pain but with minimal autonomic symptoms (Patients 1 and 2) carry the single amino acid substitution $\mathrm{R} 185 \mathrm{H}$, presenting the opportunity to compare variants associated with the extreme ends of a spectrum from minimal to severe autonomic dysfunction. In this study, we hypothesized that the difference in autonomic symptoms in patients carrying the two different $\mathrm{Na}_{\mathrm{v}} 1.7$ variants reflects a neuron type-specific effect of the variant channels. Because primary sensory (DRG) and sympathetic ganglion neurons express different ensembles of channels, ${ }^{10}$ we hypothesized that $\mathrm{R} 185 \mathrm{H}$ and $1739 \mathrm{~V}$ channels might differentially alter excitability in these two neuronal types. We show here that the $\mathrm{R} 185 \mathrm{H}$ variant affects biophysical properties of the channels by enhancing resurgent current within DRG, but not SCG neurons, while the $1739 \mathrm{~V}$ variant produces a similar change in channel function of impaired slow-inactivation, in both cell types (Tables 1 and 2). We also show here that $1739 \mathrm{~V}$ renders DRG neurons hyperexcitable while rendering SCG neurons hypoexcitable, consistent with the clinical picture of pain accompanied by autonomic dysfunction in patients carrying this variant. In contrast, we show that the $\mathrm{R} 185 \mathrm{H}$ variant channel renders DRG neurons hyperexcitable without affecting the excitability of SCG neurons, consistent with the clinical picture predominated by pain with only minimal autonomic dysfunction in patients carrying this variant. Our data suggest a correlation between effects of these two $\mathrm{Na}_{\mathrm{v}} \mathrm{T} .7$ variants on sensory and sympathetic neurons and the clinical manifestations in patients carrying these variants.

The conservation of the $\mathrm{R} 185$ and the 1739 residues among $\mathrm{Na}_{\mathrm{v}} 1.7$ mammalian orthologues and the human channel paralogues is consistent with an important role for these residues, and with altered gating of the $\mathrm{R} 185 \mathrm{H}$ and $1739 \mathrm{~V}$ channel variants. The 1739 residue is highly conserved within the middle of DII/S1, and the $1739 \mathrm{~V}$ substitution impairs slow-inactivation. ${ }^{16}$ Missense substitutions of residues corresponding to $\mathrm{R}_{1} 85$ in $\mathrm{DI} / \mathrm{S}_{2}-3$ of $\mathrm{Na}_{\mathrm{v}} 1.2$ and $\mathrm{Na}_{\mathrm{v}} 1.5$ have been linked to epilepsy and $\mathrm{LQT}_{3}$ excitability disorders, respectively. $\mathrm{Na}_{\mathrm{v}}{ }^{1.5} / \mathrm{R} 190 \mathrm{Q}$ has been identified in a patient with $\mathrm{LQT}_{3}$ cardiac disorder, ${ }^{27}$ although the 
effect of this substitution on the gating properties of $\mathrm{Na}_{\mathrm{v}} \mathrm{T} .5$ have not been determined. $\mathrm{Na}_{\mathrm{v}} 1.2 / \mathrm{R} 187 \mathrm{~W}$ has been identified in a patient with seizures and the mutant channel has been shown to inactivate more slowly than wild-type channels. ${ }^{28}$ Our observations show that $\mathrm{R} 185 \mathrm{H}$ enhances resurgent currents. Changes produced by both $1739 \mathrm{~V}$ and $\mathrm{R} 185 \mathrm{H}$ variant channels are predicted to induce a hyperexcitability phenotype in DRG neurons.

Table 1. Summary functional characterization of $\mathrm{Na}_{v} 1.7 \mathrm{SFN}$ variants in DRG neurons $†$

\begin{tabular}{|c|c|c|c|c|c|}
\hline Neuronal Type & \multicolumn{4}{|c|}{ DRG } \\
\hline Channel Variant & \multicolumn{2}{|c|}{ Voltage-Clamp } & \multicolumn{3}{|c|}{ Channel Variant } \\
\hline & Resurgent Current & Slow-Inactivation & RMP & Threshold & Repetitive Firing \\
\hline $\mathrm{R} 185 \mathrm{H}$ & Increased & No Change & No Change & Reduced & Increased \\
\hline $1739 \mathrm{~V}$ & $*$ No Change & $*$ Impaired & Depolarized & Reduced & Increased \\
\hline
\end{tabular}

$\dagger$ Compared to WT channels expressed in DRG neurons *Han et al, 2012. ${ }^{16}$ RMP = resting membrane potential

Table 2. Summary functional characterization of $\mathrm{Na}_{\mathrm{v}} 1.7 \mathrm{SFN}$ variants in DRG neurons ${ }^{\dagger}$

\begin{tabular}{|c|c|c|c|c|c|}
\hline Neuronal Type & \multicolumn{4}{|c|}{ SCG } \\
\hline Channel Variant & \multicolumn{2}{|c|}{ Voltage-Clamp } & \multicolumn{2}{c|}{ Channel Variant } \\
\hline & Resurgent Current & Slow-Inactivation & RMP & Threshold & Repetitive Firing \\
\hline R $185 \mathrm{H}$ & Not Detectable & No Change & No Change & No Change & No Change \\
\hline $1739 \mathrm{~V}$ & Not Detectable & Impaired & Depolarized & Increased & Decreased \\
\hline
\end{tabular}

$\uparrow$ Compared to WT channels expressed in SCG neurons

The expression of $\mathrm{Na}_{\mathrm{v}} 1.7 / 1739 \mathrm{~V}$ in neonatal DRG neurons (this study) depolarizes the resting membrane potential, lowers threshold for action potential firing and increase repetitive firing in response to suprathreshold stimuli, effects that are similar to those we recently reported in adult DRG neurons. ${ }^{16}$ Expression of $\mathrm{R} 185 \mathrm{H}$ channels in neonatal DRG neurons also increases excitability of these cells. Although the enhanced resurgent currents in the $\mathrm{R} 185 \mathrm{H}$ variants would be predicted to increase repetitive firing, ${ }^{29,30}$ our biophysical profiling of the $\mathrm{R} 185 \mathrm{H}$ channels does not provide an explanation for the reduced action potential threshold in DRG neurons transfected with this channel variant. We detected resurgent currents in $32 \%$ of DRG neurons transfected with $\mathrm{R} 185 \mathrm{H}$, while most of these cells were hyperexcitable. It is possible that differences in age and species of the expression platform in assessment of resurgent currents (voltage-clamp in DRG neurons from 4 - to 8-week-old $\mathrm{Na}_{\mathrm{v}} 1.8 \%$ mice) and excitability (current-clamp in DRG neurons from 0 - to 5 -day-old rats) may have 
contributed to the discrepancy. Alternatively, under our recording conditions, we were able to reliably detect resurgent currents with amplitude $>100 \mathrm{pA}$ and could detect resurgent currents as small as $50 \mathrm{pA}$ if they displayed typical wave form in cells with low noise, but we could not reliably detect smaller resurgent currents. Thus, the possibility exists that the $\mathrm{R} 185 \mathrm{H}$ variant induced small but functional resurgent currents in cells where we could notdetect them, so that the estimate of resurgent current presence within $32 \%$ of cells may be an underestimate. Finally, it is possible that the $\mathrm{R} 185 \mathrm{H}$ variant alters some aspect of channel function that was not assessed by our recording protocol. Irrespective of the underlying explanation, the effects of $1739 \mathrm{~V}$ and $\mathrm{R} 185 \mathrm{H}$ channels on the excitability of small DRG neurons, which includes nociceptors, are consistent with the severe pain experienced by the patients carrying these substitutions. Expression of these two variant $\mathrm{Na}_{\mathrm{v}} \mathrm{T} .7$ channels in neonatal SCG neurons resulted in different effects on neuronal firing. Similar to DRG neurons, expression of $1739 \mathrm{~V}$ channels in SCG neurons depolarizes the resting membrane potential. However, I739V expression in SCG neurons increases threshold for action potential firing and reduces overshoot amplitude and number of action potentials fired in response to a sustained stimulus, thus rendering SCG neurons hypoexcitable. The differential effect of $1739 \mathrm{~V}$, which renders DRG neurons hyperexcitable and SCG neurons hypoexcitable, is similar to the effect of the inherited erythromelalgia mutation $\mathrm{L} 858 \mathrm{H}$, which we have previously described..$^{\circ}$ Both $1739 \mathrm{~V}$ and $\mathrm{L} 858 \mathrm{H}$ depolarize resting membrane potential of DRG and SCG neurons. This depolarization at rest appears to differentially affect the excitability of these neurons because of the presence of $\mathrm{Na}_{\mathrm{v}} 1.8$, which is relatively resistant to inactivation by depolarization, ${ }^{31-33}$ in DRG neurons and its absence in SCG neurons. ${ }^{10}$ In support of this hypothesis, we have previously shown that firing of SCG neurons expressing mutant $\mathrm{Na}_{\mathrm{v}} 1.7 / \mathrm{L} 858 \mathrm{H}$ channels can be rescued by the co-expression of $\mathrm{Na}_{\mathrm{v}} 1.8 . .^{10}$

$\mathrm{R} 185 \mathrm{H}$ channels did not produce resurgent currents or any other changes in sodium currents when expressed in SCG neurons and did not alter the resting membrane potential of either DRG or SCG neurons, and thus would not be expected to alter the excitability of SCG neurons. Indeed, $\mathrm{R} 185 \mathrm{H}$ expression did not lower the threshold or increase repetitive firing in response to sustained stimuli in SCG neurons. Enhanced resurgent current by the $\mathrm{R}_{1} 85 \mathrm{H}$ channels within DRG neurons is consistent with the hyperexcitability of DRG neurons, as has recently been shown for $\mathrm{Na}_{\mathrm{v}} \mathrm{T} .7$ mutations from patients with paroxysmal extreme pain disorder. ${ }^{26}$ Our demonstration of resurgent currents in $32 \%$ of DRG neurons expressing the $\mathrm{R} 185 \mathrm{H}$ channel is consistent with previous studies which reported that roughly half of small DRG neurons transfected with $\mathrm{Na}_{\mathrm{v}} 1.6$ channels ${ }^{34}$ or $\mathrm{Na}_{\mathrm{v}} 1.7$ channels ${ }^{26}$ produce resurgent currents. The enhanced resurgent current produced by the $\mathrm{R} 185 \mathrm{H}$ channels in DRG neurons 
may underlie increased firing of these neurons, since it has been shown that resurgent sodium currents contribute to the high frequency firing of Purkinje neurons. ${ }^{29},{ }^{30}$. Importantly, resurgent current has been shown to be critically dependent on cell background and the same sodium channel that produces a robust resurgent current in one neuronal type may not generate this current in a different neuronal type. ${ }^{29,30,34}$ Our data suggest that SCC neurons do not support the production of resurgent current, unlike DRG neurons, a difference that would contribute to the differential effect of $\mathrm{R} 185 \mathrm{H}$ on these two neuronal types.

Although our results demonstrate the $\mathrm{R} 185 \mathrm{H}$ and $1739 \mathrm{~V}$ variants in multiple patients with idiopathic small fiber neuropathy, we would stress that a causative role has not been definitively demonstrated. In contrast to rare sodium channel variants associated with disease (some unique to one family), the $\mathrm{R} 185 \mathrm{H}$ and $1739 \mathrm{~V}$ variants both appear to be present in the general population at frequencies that may be higher than the frequency of idiopathic small fiber neuropathy. Therefore, some unaffected individuals may be heterozygous for these variants and have the same genotype as the patients. Importantly, clinical manifestations of idiopathic small fiber neuropathy are usually not recognizable until adulthood, and there can be variability in age of clinical onset for patients carrying the same variant, as illustrated for the patients carrying the $1739 \mathrm{~V}$ variant reported here and by patients carrying other $\mathrm{Na}_{\mathrm{y}} 1.7$ variants. ${ }^{35}$ These considerations raise the possibility that these variants may in fact be 'risk factors' that are not sufficient to cause disease on their own, but may contribute to development of idiopathic small fiber neuropathy in combination with other genetic or environmental factors/stressors.

We show here that while the $1739 \mathrm{~V}$ channels depolarize resting membrane potential in both cell types, they render DRG neurons hyperexcitable and SCG neurons hypoexcitable. The opposite functional effects of the $1739 \mathrm{~V}$ on neuronal excitability are likely to be caused by the selective expression of $\mathrm{Na}_{\mathrm{v}} 1.8$ in DRG but not SCG neurons. In contrast, $\mathrm{R} 185 \mathrm{H}$ enhances resurgent current and renders DRG neurons hyperexcitable, but does not alter excitability of SCC neurons, perhaps due to inability of these neurons to produce the resurgent current. Our observations on the differential effects of the $\mathrm{R} 185 \mathrm{H}$ and $1739 \mathrm{~V}$ $\mathrm{Na}_{\mathrm{v}}$. 7 variants on DRG and SCG neurons provide a demonstration that sodium channel variants can have a spectrum of cell-background-dependent effects in different types of neurons. Our results suggest that these differential effects in different types of neurons can contribute to clinical phenotype, in this case explaining the presence of profound autonomic dysfunction in patients carrying the $1739 \mathrm{~V}$ variant, and minimal autonomic dysfunction in patients carrying the $\mathrm{R} 185 \mathrm{H}$ variant. 


\section{References}

1. Stewart, J.D., Low, P.A. \& Fealey, R.D. Distal small fiber neuropathy: results of tests of sweating and autonomic cardiovascular reflexes. Muscle Nerve 15, 661-5 (1992).

2. Gorson, K.C. \& Ropper, A.H. Idiopathic distal small fiber neuropathy. Acta Neurol Scand 92, 376-82 (1995).

3. Holland, N.R. et al. Small-fiber sensory neuropathies: clinical course and neuropathology of idiopathic cases. Ann Neurol 44, 47-59 (1998).

4. Lacomis, D. Small-fiber neuropathy. Muscle Nerve 26, 173-88 (2002).

5. Lauria, G. Small fibre neuropathies. Curr Opin Neurol 18, 591-7 (2005).

6. Devigili, G. et al. The diagnostic criteria for small fibre neuropathy: from symptoms to neuropathology. Brain 131, 1912-25 (2008).

7. Bednarik, J. et al. Etiology of small-fiber neuropathy. J Peripher Nerv Syst 14, 177-83 (2009).

8. Faber, C.G. et al. Gain of function $\mathrm{Na}(\mathrm{V}) 1.7$ mutations in idiopathic small fiber neuropathy. Ann Neurol 71, 26-39 (2012).

9. Toledo-Aral, J.). et al. Identification of $\mathrm{PN}_{1}$, a predominant voltage-dependent sodium channel expressed principally in peripheral neurons. Proc Natl Acad Sci U S A 94, 1527-32 (1997).

10. Rush, A.M. et al. A single sodium channel mutation produces hyper- or hypoexcitability in different types of neurons. Proc Natl Acad Sci U S A 103, 8245-50 (2006).

11. Persson, A.K. et al. Sodium-calcium exchanger and multiple sodium channel isoforms in intra-epidermal nerve terminals. Mol Pain 6, 84 (2010).

12. Cummins, T.R., Howe, J.R. \& Waxman, S.G. Slow closed-state inactivation: a novel mechanism underlying ramp currents in cells expressing the hNE/PNi sodium channel. J Neurosci 18, 9607-19 (1998).

13. Dib-Hajj, S.D. et al. Gain-of-function mutation in Nav1.7 in familial erythromelalgia induces bursting of sensory neurons. Brain 128, 1847-54 (2005).

14. Dib-Hajj, S.D., Cummins, T.R., Black, J.A. \& Waxman, S.G. Sodium channels in normal and pathological pain. Annu Rev Neurosci 33, 325-47 (2010).

15. Fertleman, C.R. et al. SCNgA mutations in paroxysmal extreme pain disorder: allelic variants underlie distinct channel defects and phenotypes. Neuron 52, 767-74 (2006).

16. Han, C. et al. Nav1.7-related small fiber neuropathy: impaired slow-inactivation and DRG neuron hyperexcitability. Neurology 78, 1635-43 (2012).

17. Lauria, G. et al. European Federation of Neurological Societies/Peripheral Nerve Society Guideline on the use of skin biopsy in the diagnosis of small fiber neuropathy. Report of a joint task force of the European Federation of Neurological Societies and the Peripheral Nerve Society. Eur J Neurol 17, 903-12, e44-9 (2010).

18. Shy, M.E. et al. Quantitative sensory testing: report of the Therapeutics and Technology Assessment Subcommittee of the American Academy of Neurology. Neurology 60, 898-904 (2003).

19. Yarnitsky, D. \& Sprecher, E. Thermal testing: normative data and repeatability for various test algorithms. J Neurol Sci 125, 39-45 (1994).

20. Hoitsma, E. et al. Abnormal warm and cold sensation thresholds suggestive of small-fibre neuropathy in sarcoidosis. Clin Neurophysiol 114, 2326-33 (2003).

21. Drenth, J.P. et al. SCNgA mutations define primary erythermalgia as a neuropathic disorder of voltage gated sodium channels. J Invest Dermatol 124, 1333-8 (2005).

22. Klugbauer, N., Lacinova, L., Flockerzi, V. \& Hofmann, F. Structure and functional expression of a new member of the tetrodotoxin-sensitive voltage-activated sodium channel family from human neuroendocrine cells. EMBOJ 14, 1084-90 (1995).

23. Stirling, L.C. et al. Nociceptor-specific gene deletion using heterozygous NaV1.8-Cre recombinase mice. Pain 113, 27-36 (2005).

24. Dib-Hajj, S.D. et al. Transfection of rat or mouse neurons by biolistics or electroporation. Nat Protoc 4 $1118-26$ (2009).

25. Herzog, R.I., Cummins, T.R., Ghassemi, F., Dib-Hajj, S.D. \& Waxman, S.G. Distinct repriming and closedstate inactivation kinetics of Nav1. 6 and Nav1.7 sodium channels in mouse spinal sensory neurons. J Physiol 551, 741-50 (2003). 
26. Jarecki, B.W., Piekarz, A.D., Jackson, J.O., 2nd \& Cummins, T.R. Human voltage-gated sodium channel mutations that cause inherited neuronal and muscle channelopathies increase resurgent sodium currents. J Clin Invest 120, 369-78 (2010).

27. Chung, S.K. et al. Long QT and Brugada syndrome gene mutations in New Zealand. Heart Rhythm 4, 1306-14 (2007).

28. Sugawara, T. et al. A missense mutation of the $\mathrm{Na}$ + channel alpha II subunit gene $\mathrm{Na}(\mathrm{v}) 1.2$ in a patient with febrile and afebrile seizures causes channel dysfunction. Proc Natl Acad Sci U S A 98, 6384-9 (2001).

29. Raman, I.M. \& Bean, B.P. Resurgent sodium current and action potential formation in dissociated cerebellar Purkinje neurons. J Neurosci 17, 4517-26 (1997).

30. Raman, I.M., Sprunger, L.K., Meisler, M.H. \& Bean, B.P. Altered subthreshold sodium currents and disrupted firing patterns in Purkinje neurons of Scn8a mutant mice. Neuron 19, 881-91 (1997).

31. Akopian, A.N., Sivilotti, L. \& Wood, J.N. A tetrodotoxin-resistant voltage-gated sodium channel expressed by sensory neurons. Nature 379, 257-62 (1996).

32. Akopian, A.N. et al. The tetrodotoxin-resistant sodium channel SNS has a specialized function in pain pathways. Nat Neurosci 2, 541-8 (1999).

33. Sangameswaran, L. et al. Structure and function of a novel voltage-gated, tetrodotoxin-resistant sodium channel specific to sensory neurons. J Biol Chem 271, 5953-6 (1996).

34. Cummins, T.R., Dib-Hajj, S.D., Herzog, R.I. \& Waxman, S.G. Nav1.6 channels generate resurgent sodium currents in spinal sensory neurons. FEBS Lett 579, 2166-70 (2005).

35. Estacion, M. et al. Intra- and interfamily phenotypic diversity in pain syndromes associated with a gainof-function variant of $\mathrm{NaV}$ 1.7. Mol Pain 7, 92 (2011) 

Chapter 7

\title{
Small nerve fibers, small hands
} and small feet: new syndrome of pain, dysautonomia and acromesomelia in a kindred with a novel $\mathrm{Na}_{\mathrm{v}} \mathrm{1} .7$ mutation

\author{
J.G.J. Hoeijmakers ${ }^{a *}$, C. Han ${ }^{b, c *}$, I.S.J. Merkies ${ }^{a, d}$, L.J. Macala ${ }^{b, c}$, \\ G. Lauria ${ }^{e}$, M.M. Gerrits, S.D. Dib-Hajb,c, C.G. Faber, S.G. Waxman ${ }^{b, c}$. \\ * co-first authorship
}

aDepartment of Neurology, Maastricht University Medical Center, Maastricht ${ }^{b}$ Department of Neurology, Yale University School of Medicine, New Haven, CT, USA 'Center for Neuroscience and Regeneration Research, Veterans Affairs Medical Center,

West Haven, CT, USA

${ }^{\mathrm{d} D e p a r t m e n t}$ of Neurology, Spaarne Hospital, Hoofddorp eNeuromuscular Diseases Unit, IRCCS Foundation, Carlo Besta Neurological Institute, Milan, Italy fDepartment of Clinical Genomics, Maastricht University Medical Center, Maastricht

Brain. 2012;135(Pt 2):345-58; Published with permission from Oxford Journals 


\section{Abstract}

The $\mathrm{Na}_{\mathrm{v}} 1.7$ sodium channel is preferentially expressed within dorsal root ganglion and sympathetic ganglion neurons and their small-diameter peripheral axons. Gain-of-function variants of $\mathrm{Na}_{\mathrm{v}} \mathrm{\gamma} .7$ have recently been described in patients with painful small fiber neuropathy and no other apparent cause. Here, we describe a novel syndrome of pain, dysautonomia, small hands and small feet in a kindred carrying a novel $\mathrm{Na}_{\mathrm{v}} 1.7$ mutation. A 35-year-old male presented with erythema and burning pain in the hands since early childhood, later disseminating to the feet, cheeks and ears. He also experienced progressive muscle cramps, profound sweating, bowel disturbances (diarrhea or constipation), episodic dry eyes and mouth, hot flashes, and erectile dysfunction. Neurological examination was normal. Physical examination was remarkable in revealing small hands and feet (acromesomelia). Blood examination and nerve conduction studies were unremarkable. Intraepidermal nerve fiber density was significantly reduced compared to age- and sex-matched normative values. The patient's brother and father reported similar complaints including distal extremity redness and pain, and demonstrated comparable distal limb under-development. Quantitative sensory testing revealed impaired warmth sensation in the proband, father and brother. Genetic analysis revealed a novel missense mutation in the SCN9A gene encoding sodium channel $\mathrm{Na}_{\mathrm{v}} 1.7$ (G856D; C.2567G>A) in all three affected subjects, but not in unaffected family members. Functional analysis demonstrated that the mutation hyperpolarizes $(-9.3 \mathrm{mV})$ channel activation, depolarizes $(+6.2 \mathrm{mV})$ steady-state fast-inactivation, slows deactivation and enhances persistent current and the response to slow ramp stimuli by 10- to 11fold compared with wild-type $\mathrm{Na}_{\mathrm{v}} \mathrm{T} .7$ channels. Current-clamp analysis of dorsal root ganglion neurons transfected with G856D mutant channels demonstrated depolarized resting potential, reduced current threshold, increased repetitive firing in response to suprathreshold stimulation and increased spontaneous firing. Our results demonstrate that the G856D mutation produces DRG neuron hyperexcitability which underlies pain in this kindred, and suggest that small peripheral nerve fiber dysfunction due to this mutation may have contributed to distal limb under-development in this novel syndrome. 


\section{Introduction}

Small fiber neuropathy is a disorder of thinly myelinated and unmyelinated nerve fibers and is clinically characterized by burning pain and autonomic complaints. ${ }^{1-4}$ The diagnosis of pure small fiber neuropathy, in which smalldiameter nerve fibers are affected but large-diameter fibers are spared, is usually made on the basis of the clinical picture, together with preservation of large fiber functions (normal strength, tendon reflexes and vibration sense) and normal nerve conduction studies, and is confirmed by demonstration of reduced intraepidermal nerve fiber density or abnormal quantitative sensory testing. ${ }^{.}$We have recently described the presence of novel functional variants in $\mathrm{SCNgA}$, the gene encoding for the $\mathrm{Na}_{\mathrm{v}} \mathrm{l} .7$ sodium channel, in $\sim 30 \%$ of patients with biopsy confirmed idiopathic small fiber neuropathy. ${ }^{6} \mathrm{Na}_{\mathrm{v}}{ }^{1.7}$ is preferentially and abundantly expressed within dorsal root ganglion (DRG) and sympathetic ganglion neurons $\mathrm{s}^{7,8}$ and their small diameter peripheral axons ${ }^{9}$ where it amplifies small depolarizations and modulates excitability. ${ }^{10}$ The biophysical changes in $\mathrm{Na}_{\mathrm{v}} \mathrm{T}$.7 channels produced by $\mathrm{SCN}$ gA-related small fiber neuropathy variants published to date include impaired slow-inactivation, impaired slow- and fastinactivation, or enhanced resurgent currents. ${ }^{6}$ In this report, we describe a kindred with clinical features of small fiber neuropathy that produced distal extremity pain, together with a remarkable phenotype of small forearms, hands, lower legs and feet (acromesomelia). Each of the three affected subjects harboured a novel mutation in $\mathrm{SCNgA}(\mathrm{c} .2567 \mathrm{G}>\mathrm{A}$; $\mathrm{G} 856 \mathrm{D}$ ) that produces an ensemble of functional changes including enhanced activation, ramp current and persistent current. The mutant channels increase DRG neuron excitability. Our results demonstrate that the G856D mutation produces DRG neuron hyperexcitability that underlies pain in this kindred, and suggest the hypothesis that this $\mathrm{Na}_{\mathrm{v}} \mathrm{l} .7$ mutation produces peripheral nerve dysfunction that may contribute to limb underdevelopment.

\section{Materials and methods}

\section{Anthropometrics}

Twelve anthropometric body dimensions were measured in the index patient and family members (father, mother and two brothers) and compared with reported age- and gender-matched Dutch healthy controls (see Supplementary Fig. 1 for definitions of the measurements). ${ }^{11,12}$ All measurements were performed twice to assure accuracy. Measurements were completed by one observer (J.G.J.H.). Stature was measured using a wall-attached ruler $(\mathrm{cm})$. The subject was requested to stand straight-up against the wall, feet together and barefooted. 
A tape measure $(\mathrm{cm})$ was used for all other variables. Shoulder height sitting, elbow height sitting, elbow-grip length, buttock-popliteal distance, buttockknee distance and popliteal height were measured with the subject sitting straight-up, with the posterior parts of the buttocks against the back of a chair, the popliteal fossa against the front of the chair, knees $90^{\circ}$ flexed and the feet resting on the floor (patient sitting in a vertical plane adjustable chair). The shoulders were relaxed with the upper arms hanging alongside the body, and forearms in a horizontal plane (Supplementary Fig. 1). Hand length and hand width (with and without thumb) were measured while the hand was extended by the subject and resting on an examination couch. Foot length and foot width were measured with the subject standing straight-up, the feet slightly apart and weight evenly distributed. All findings were compared with published normative values. ${ }^{11,} 12$

\section{Quantitative sensory testing}

Quantitative sensory testing was performed as previously described.6, 13-16 In brief, quantitative sensory testing thresholds for warm, cool and heat pain modalities were assessed using a TSH-2001 device (Medoc) at the dorsum of both feet and thenar eminences, and a modality was classified as abnormal if results of both method-of-limits and method-of-levels were abnormal. ${ }^{13,14}$

\section{Intraepidermal nerve fiber density}

For assessment of intraepidermal nerve fiber density, a punch biopsy was taken $10 \mathrm{~cm}$ above the lateral malleolus. The number of individual nerve fibers crossing the dermal-epidermal junction was counted in three randomly taken sections $(50 \mu \mathrm{m})$ after fixing the biopsy by subjecting it to cryoprotective solution and immunostaining with polyclonal rabbit anti-protein-gene-product-9.5 antibody (PGP9.5; Ultraclone), using bright-field microscopy with a stereology workstation (Olympus BX50, PlanApo oil-objective $\mathrm{X}_{40} / \mathrm{NA}=1.0$ ). ${ }^{17}$ Intraepidermal nerve fiber density findings were compared with published normative data. ${ }^{7}$

\section{SCNgA mutation analysis}

Genomic DNA was extracted from blood using the Puregene (C) genomic DNA isolation kit (Gentra-Systems). All coding exons and flanking intronic sequences, and exons encoding $5^{\prime}$ and $3^{\prime}$ untranslated sequences within the complementary DNA, were amplified and sequenced as described previously. ${ }^{18}$ Genomic sequences were compared with reference $\mathrm{Na}_{\mathrm{v}} 1.7$ complementary DNA (NM_002977. $3^{19}$ ) to identify sequence variations, using Alamut MutationInterpretation Software (Interactive-Biosoftware). A control panel of DNA from 100 healthy Dutch (Caucasian) individuals (200 chromosomes) was screened for all new mutations. The NCBI SNP database, the Human Gene Mutation 
Database (HGMDC) and the 1000 genomes project (www.10oogenomes.org/ data) were also screened.

\section{Functional analysis}

Voltage-clamp analysis

The plasmid carrying the tetrodotoxin-resistant version of human $\mathrm{Na}_{\mathrm{v}} 1.7$ complementary DNA $\left(\mathrm{hNa}_{\mathrm{v}} 1.7_{\mathrm{R}}\right)$ was described previously. ${ }^{10}$ The $\mathrm{G} 856 \mathrm{D}$ mutation was introduced into $h \mathrm{ha}_{\mathrm{V}} 1 \cdot 7_{\mathrm{R}}$ using QuickChange $(\mathrm{C} \mathrm{XL}$ site-directed mutagenesis (Stratagene). Wild-type or G856D mutant $h \mathrm{ha}_{\mathrm{v}} 1.7_{R}$ channels were co-transfected with the human $\beta_{1}$ and $\beta_{2}$ subunits into HEK293 cells, grown under standard culture conditions $\left(5 \% \mathrm{CO}_{2}, 37^{\circ} \mathrm{C}\right)$ in Dulbecco's Modified Eagle's Medium supplemented with $10 \%$ foetal bovine serum, by Optifect ${ }^{\mathrm{TM}}$ transfection reagent (Invitrogen).

Whole-cell patch-clamp recordings were obtained at room temperature $\left(\sim 21^{\circ} \mathrm{C}\right)$, $24 \mathrm{~h}$ after transfection using an EPC-9 amplifier and Pulse 8.5 (HEKA) with $0.8-1.5 \mathrm{M} \Omega$ electrodes. Voltage errors were minimized using $80-90 \%$ series resistance compensation and linear leak subtraction; capacitance artefact was cancelled using computer-controlled circuitry. Recordings were started 5 min after establishing whole-cell configuration. The pipette solution contained: $140 \mathrm{mM}$ CsF, $1 \mathrm{mM}$ EGTA, $10 \mathrm{mM} \mathrm{NaCl}$ and $10 \mathrm{mM}$ HEPES, pH 7.3 with $\mathrm{CsOH}$ (adjusted to $315 \mathrm{mOsm}$ with dextrose). The bath solution was $140 \mathrm{mM} \mathrm{NaCl}$, $3 \mathrm{mM} \mathrm{KCl}, 1 \mathrm{mM} \mathrm{MgCl}, 1 \mathrm{mM} \mathrm{CaCl}$ and $10 \mathrm{mM}$ HEPES, $\mathrm{pH} 7.3$ with $\mathrm{NaOH}$ (adjusted to $320 \mathrm{mOsm}$ with dextrose).

\section{Transfection of dorsal root ganglion neurons and current-clamp recordings}

The protocol for care and sacrifice of rats used in the study was approved by the Veterans Administration Connecticut Healthcare system IACUC. DRG from 4- to 8-week-old Sprague-Dawley rats were harvested and dissociated as previously described. ${ }^{20}$ Sodium channel and Green Flourescent Protein (GFP) constructs (channel:GFP ratio of 5:1) were electroporated into DRG neurons using Rat Neuron Nucleofector Solution (Amaxa) with wild-type $\mathrm{Na}_{\mathrm{v}} 1.7_{\mathrm{R}}$, and G856D mutant derivative as described previously. ${ }^{21}$ Transfected DRG neurons were incubated at $37^{\circ} \mathrm{C}$ in $\mathrm{Ca}^{2+}$-free culture medium (Dulbecco's Modified Eagle's Medium) for 5 min to increase cell viability. The cell suspension was then diluted in culture medium supplemented with nerve growth factor and glial cell line-derived neurotrophic factor $(50 \mathrm{ng} / \mathrm{ml})$, plated on $12 \mathrm{~mm}$ circular coverslips coated with laminin and poly-lysine and incubated at $37^{\circ} \mathrm{C}$ in $5 \% \mathrm{CO}_{2}$. Current-clamp recordings were obtained from small (22-28 $\mu \mathrm{m}$ diameter) GFPlabelled DRG neurons $40-48 \mathrm{~h}$ after transfection. Electrodes had a resistance of $1-3 \mathrm{M} \Omega$ when filled with the pipette solution, which contained the following (in $\mathrm{mM}$ ): $140 \mathrm{KCl}$, 0.5 EGTA, 5 HEPES and $3 \mathrm{Mg}$-ATP, pH 7.3 with $\mathrm{KOH}$ (adjusted to 
$315 \mathrm{mOsm}$ with dextrose). The extracellular solution contained the following (in $\mathrm{mM}$ ): $140 \mathrm{NaCl}, 3 \mathrm{KCl}, 2 \mathrm{MgCl}_{2}, 2 \mathrm{CaCl}_{2}, 10 \mathrm{HEPES}, \mathrm{pH} 7.3$ with $\mathrm{NaOH}$ (adjusted to $320 \mathrm{mOsm}$ with dextrose). Whole-cell configuration was obtained in voltageclamp mode before proceeding to the current-clamp recording mode. Cells with stable ( $<10 \%$ variation) resting membrane potentials more negative than $-35 \mathrm{mV}$ were used for data collection. Threshold was determined by the first action potential elicited by a series of depolarizing current injections that increased in $5 \mathrm{pA}$ increments.

\section{Study design}

The study was approved by medical ethical committees at Yale University and Maastricht University Medical Center. All aspects of the study were explained to the patient and family members and written informed consent and permission for use of photographs were obtained.

\section{Data analysis}

Electrophysiological data were analysed using PulseFit 8.74 (HEKA Electronics) and Origin 8.1 (Microcal), and presented as means \pm standard error. Statistical significance was determined by unpaired Student's $t$-tests (current-clamp except firing frequency and spontaneous activity), Mann-Whitney test (firing frequency) or two proportion z-test (comparison of proportion of cells producing spontaneous activity).

\section{Results}

\section{Clinical description}

A 35-year-old male presented with episodic cramps, erythema and burning pain. At the age of 10 years, he experienced episodes of redness of the skin in both hands that lasted for hours. A few years later, he developed burning pain in his hands. These symptoms were triggered by warmth, exercise or taking a warm bath, and were relieved by cold water. In adulthood, pain episodes were also triggered by ingestion of alcohol. Initially, the episodes occurred in the evening. However, there was a gradual increase in frequency and duration of pain episodes, which could occur at any time during the day. The redness of the skin and the pain also expanded to the feet, cheeks and ears. The intensity and hotness ('burning') of pain were scored 8 and 9, respectively, on a 10-point scale. ${ }^{22}$ The patient also experienced episodic spontaneous sweating, bowel disturbances (diarrhea or constipation), episodic dry eyes and mouth, hot flashes, and erectile dysfunction. The patient did not use any drugs that are known to cause neuropathy. 
Treatment with aspirin, a beta-blocker and pregabalin were ineffective.

On physical examination, no signs of large nerve fiber involvement were found. As shown in Fig. 1 A, the patient had profound redness of the distal forearms and hands. Remarkably, his hands, lower legs and feet were small when compared to age- and gender-matched normative values (Figs $1 B$ and 2; Table 1; see Supplementary Fig. 1 for explanation of anthropometric measurements). ${ }^{\text {" }}$ 12 Nerve conduction studies were normal. The patient was given a clinical diagnosis of small fiber neuropathy and was subsequently subjected to additional investigations. Tables 2 and 3 summarize the clinical findings of the index patient and family members.

The proband's father (aged 68 years at time of examination) experienced redness of the feet and hands until his early twenties. After that age, the complaints resolved and never re-appeared. At the time of work-up, he noted only occasional restless legs. The proband's brother (aged 32 years at the time of examination; indexed as Brother A) complained of redness of the extremities, hands and feet with painful episodes triggered by exposure to high temperatures. He had intolerance to socks and sheets over his feet and complained of intermittent dry mouth and eyes, postural dizziness and episodic palpitations (Tables 2 and 3 ). Physical examination was notable for small distal forearms, hands, distal legs and feet in the proband's father and Brother A (Fig. 3 and Table 1). Neurological examination was unremarkable. The proband's mother and another brother (indexed as Brother B) had no complaints and no physical abnormalities (including normal anthropometrics). 
Table 1. Body proportions: findings of index patient and family members with G856D SCNgA mutation.

\begin{tabular}{|c|c|c|c|c|}
\hline $\begin{array}{l}\text { Anthropometric } \\
\text { measurements } \\
\text { (see figure e-1 } \\
\text { for explanation) }\end{array}$ & Measure & $\begin{array}{l}\text { Mean }(\mathrm{mm}) \\
(31-60 \text { years })\end{array}$ & $\begin{array}{c}5^{\text {th }} \text { percentile cutoff } \\
\text { (31-60 years) }\end{array}$ & $\begin{array}{c}\text { Index patient }(\mathrm{mm}) / \\
\% \text { value }\end{array}$ \\
\hline 1 & Stature & 1770 & 1645 & $1665 / 8.1 \%$ \\
\hline 2 & Shoulder height sitting & 612 & 561 & $580 / 15.1 \%$ \\
\hline 3 & Elbow height sitting & 253 & 207 & $235 / 26.5 \%$ \\
\hline 4 & Elbow-grip length & 352 & 318 & $340 / 28.5 \%$ \\
\hline 5 & Hand length & 194 & 178 & $160 / 0.1 \%$ \\
\hline 6 & Hand width (with thumb) & 119 & 111 & $90 / 0.1 \%$ \\
\hline 7 & Hand width without thumb & 91 & 84 & $70 / 0.1 \%$ \\
\hline 8 & Buttock-popliteal distance & 503 & 459 & $450 / 2.4 \%$ \\
\hline 9 & Buttock-knee distance & 631 & 579 & $510 / 0.1 \%$ \\
\hline 10 & Popliteal height & 481 & 429 & $370 / 0.1 \%$ \\
\hline 11 & Foot length & 266 & 241 & $180 / 0.1 \%$ \\
\hline 12 & Foot width & 101 & 91 & $70 / 0.1 \%$ \\
\hline
\end{tabular}

Legend to table 1 . The numbers in bold are lower than the $5^{\text {th }}$ percentile cut-off normative values ${ }^{12}$.

Table 2. Clinical description of index patient and family members with G856D SCN9A mutation.

\begin{tabular}{|c|c|c|c|c|c|}
\hline $\begin{array}{c}\text { Age at } \\
\text { referral/ } \\
\text { Gender }\end{array}$ & $\begin{array}{c}\text { Age at } \\
\text { onset } \\
\text { symptoms } \\
\text { patient }\end{array}$ & $\begin{array}{c}\text { Initial } \\
\text { symptom(s) } \\
\text { + location }\end{array}$ & Later symptoms & \\
\hline Brother A & $35 /$ male & 10 & $\begin{array}{c}\text { Redness hands } \\
\text { and wrists, } \\
\text { burning pain }\end{array}$ & $\begin{array}{c}\text { Beginning at age } 25 \text { years, redness skin } \\
\text { expanded to the feet, cheeks and } \\
\text { ear region and became painful }\end{array}$ & \\
\hline Father & $68 /$ male & $\begin{array}{c}\text { Childhood } \\
\text { Cntil age } 20\end{array}$ & $\begin{array}{c}\text { Redness hands } \\
\text { and feet } \\
\text { and feet }\end{array}$ & Recent onset moderate burning pain & \\
\hline
\end{tabular}

Legend to table 2. Quantitative sensory testing scores were compared with normative values. ${ }^{23} \mathrm{~A}$ sensory modality was classified as abnormal if the results of both method-of-limits and methodof-levels were abnormal. Note: the mother and a second brother (indexed as Brother B) did not have complaints, had no physical abnormalities (normal anthropometrics) and did not harbour the G856D mutation. $L=$ left; $R=$ right; $\uparrow=$ increase; $\downarrow=$ reduced. 


\begin{tabular}{|c|c|c|c|c|}
\hline & $\begin{array}{c}\text { Proband's brother }(\mathrm{mm}) / \\
\% \text { value }\end{array}$ & $\begin{array}{c}\text { Mean }(\mathrm{mm}) \\
(>60 \text { years })\end{array}$ & $\begin{array}{c}5^{\text {th }} \text { percentile cutoff } \\
(>60 \text { years })\end{array}$ & $\begin{array}{c}\text { Proband's father (mm) / } \\
\% \text { value }\end{array}$ \\
\hline & $1760 / 45.3 \%$ & 1729 & 1619 & $1610 / 3.9 \%$ \\
\hline & $550 / 2.2 \%$ & 596 & 545 & $510 / 0.3 \%$ \\
\hline & $180 / 0.5 \%$ & 237 & 189 & $170 / 1 \%$ \\
\hline & $340 / 28.5 \%$ & 347 & 317 & $330 / 17.1 \%$ \\
\hline & $175 / 2.8 \%$ & 191 & 175 & $180 / 13.8 \%$ \\
\hline & $90 / 0.1 \%$ & 111 & 103 & $110 / 42 \%$ \\
\hline & $70 / 0.1 \%$ & 90 & 82 & $80 / 2.2 \%$ \\
\hline & $500 / 44.7 \%$ & 499 & 458 & $470 / 12.5 \%$ \\
\hline & $590 / 10.4 \%$ & 625 & 579 & $570 / 2.4 \%$ \\
\hline & $430 / 5.4 \%$ & 473 & 427 & $440 / 11.7 \%$ \\
\hline & $210 / 0.1 \%$ & 267 & 246 & $240 / 1.8 \%$ \\
\hline & $80 / 0.1 \%$ & 100 & 90 & $90 / 4.8 \%$ \\
\hline
\end{tabular}

Precipitating factors/ Relieving factors

Medication

IENFD +

(corresponding normative value)

QST impaired modality

SCNgA

mutation

\begin{tabular}{|c|c|c|c|c|c|}
\hline & & 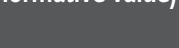 & Thenar & Foot & \\
\hline $\begin{array}{c}\uparrow \text { with warmth, exercise, } \\
\text { or drinking alcohol. } \\
\downarrow \text { by cooling. }\end{array}$ & $\begin{array}{c}\text { No effect } \\
\text { Aspirin, } \\
\text { beta-blocker } \\
\text { and } \\
\text { Pregabalin }\end{array}$ & $\begin{array}{c}5.0 / \mathrm{mm} \\
(\geq 5.2 / \mathrm{mm})\end{array}$ & $\begin{array}{l}\text { Warmth-R } \\
\text { Warmth-L }\end{array}$ & & $\begin{array}{c}\text { c. } 2567 \mathrm{G}>\mathrm{A}, \\
\mathrm{G} 856 \mathrm{D}\end{array}$ \\
\hline$\uparrow$ rising temperatures & - & $\begin{array}{c}11.1 / \mathrm{mm} \\
(\geq 5.2 / \mathrm{mm})\end{array}$ & Warmth-L & - & $\begin{array}{c}c .2567 \mathrm{G}>\mathrm{A}, \\
\mathrm{G} 856 \mathrm{D}\end{array}$ \\
\hline- & - & $\begin{array}{c}3.7 / \mathrm{mm} \\
(\geq 2.8 / \mathrm{mm})\end{array}$ & $\begin{array}{l}\text { Warmth-R } \\
\text { Warmth-L } \\
\text { Cold-L }\end{array}$ & Cold-R & $\begin{array}{c}c .2567 \mathrm{G}>A, \\
G 856 \mathrm{D}\end{array}$ \\
\hline
\end{tabular}


Table 3. Small nerve fiber neuropathy symptoms inventory questionnaire (SFN-SIQ) findings

\begin{tabular}{|c|c|c|c|c|c|c|c|}
\hline & sweating & diarrhea & constipation & $\begin{array}{c}\text { micturation } \\
\text { problems }\end{array}$ & dry eyes & dry mouth & \\
\hline Index patient & 1 & 1 & 1 & 1 & 1 & 1 & \\
\hline Brother A & 1 & 0 & 0 & 0 & 1 & 1 & \\
\hline Father & 0 & 0 & 0 & 0 & 0 & 0 & \\
\hline
\end{tabular}

Legend to table 3. A green indicates absence (score o) of corresponding SFN related complaint; a red box indicates the presence of SFN related symptom, with variable intensity (score 1: sometimes present; score 2: often, and a score 3: always present). Note: The proband's father (current age 68 years) had experienced redness of the feet and hands with pain until his early twenties.

Figure 1.

A
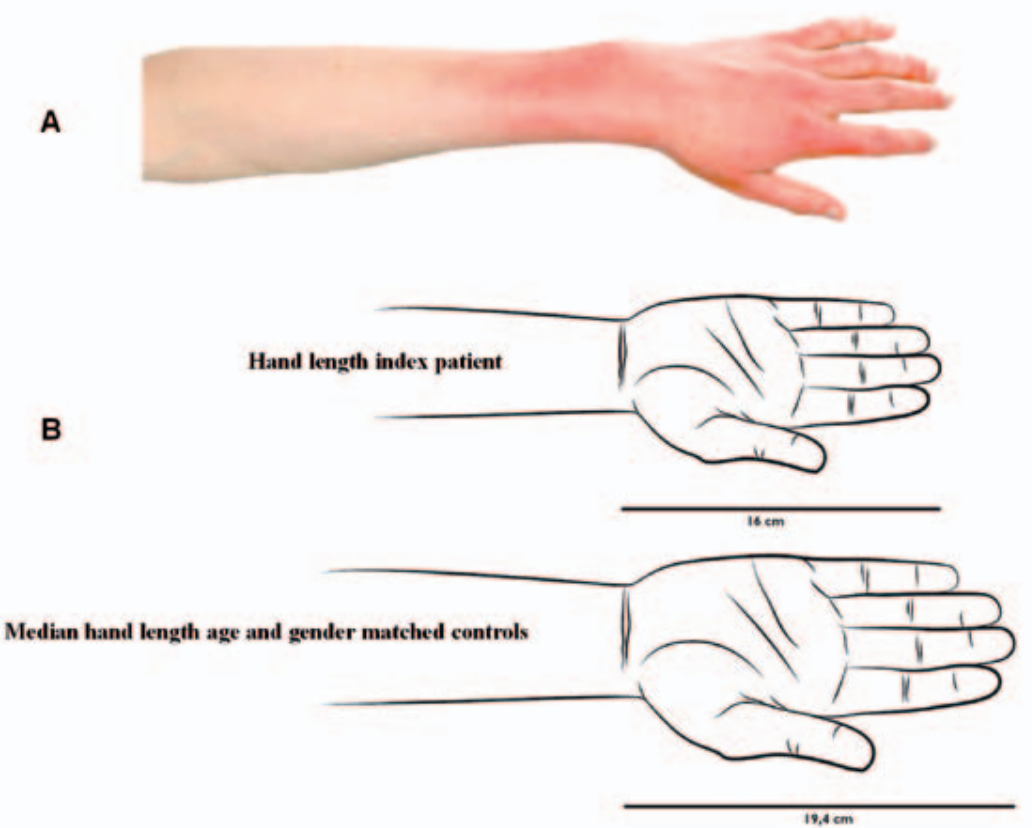

Legend to figure 1. Index patient's hand. Note the small size and distal redness of the hand (A). (B) Line drawing showing, on the same scale, the patient's hand, juxtaposed to an ageand gendermatched hand. Hand length index was assessed from the wrist crease on the palmar surface to the tip of the middle finger, parallel to the fingers. See Table 1 and Supplementary Fig. 1 for additional anthropometric findings ${ }^{12}$. 


\begin{tabular}{|c|c|c|c|c|c|c|c|}
\hline & $\begin{array}{c}\text { orthostatic } \\
\text { dizziness }\end{array}$ & palpitations & hot flashes & $\begin{array}{c}\text { skin } \\
\text { hyperesthesia }\end{array}$ & burning feet & $\begin{array}{c}\text { sheet } \\
\text { intolerance }\end{array}$ & restless legs \\
\hline & 0 & 0 & 2 & 1 & 1 & 1 & 2 \\
\hline & 1 & 1 & 0 & 1 & 2 & 1 & 2 \\
\hline
\end{tabular}

\section{Supplementary figure 1.}
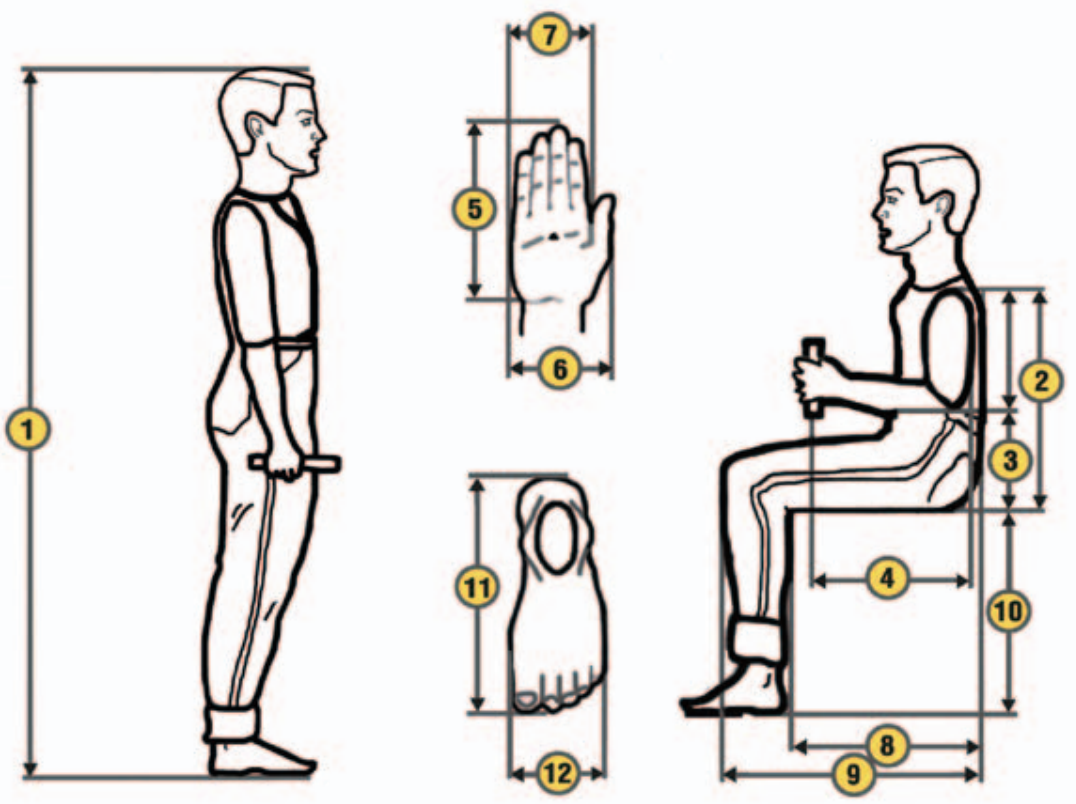

Legend to supplementary figure 1. 1:Stature (vertical distance from the floor to the top of the head using a wall-attached ruler), 2: Shoulder height sitting (vertical distance from the seat to the acromion), 3: Elbow height sitting (vertical distance from the sitting surface to the lowest bony point of the elbow, while forearm in horizontal plane), 4: Elbow-grip length (horizontal distance between the lowest bony point of the flexed elbow and the middle of the grip device), 5: Hand length (distance from the wrist crease to the tip of the middle finger, parallel to the fingers, hand stretched and resting on examination couch), 6: Hand width (with thumb) (distance from the radial to the ulnar side of the hand, measured at the distal interphalangeal joint of the thumb), 7 : Hand width without thumb (distance from the radial to the ulnar side of the hand, measured at the distal extremities of the metacarpal bones), 8: Buttock-popliteal distance (horizontal distance from the back of the chair to the popliteal fossa of the knee parallel to the long axis of the upper leg), 9: Buttock-knee distance (horizontal distance from the posterior surface of the buttock to the anterior surface of the knee parallel to the long axis of the upper leg), 10: Popliteal height (vertical distance from the floor to the popliteal fossa of the knee), 11: Foot length (maximum horizontal distance from the heel to the longest toe of the foot, parallel to the long axis of the foot), 12: Foot width (maximum width across the ball of the foot barefooted). Modified with permission by Dr. Johan F.M. Molenbroek, Technical University Delft, the Netherlands (www.dined.nl) ${ }^{12}$. 
Figure 2.

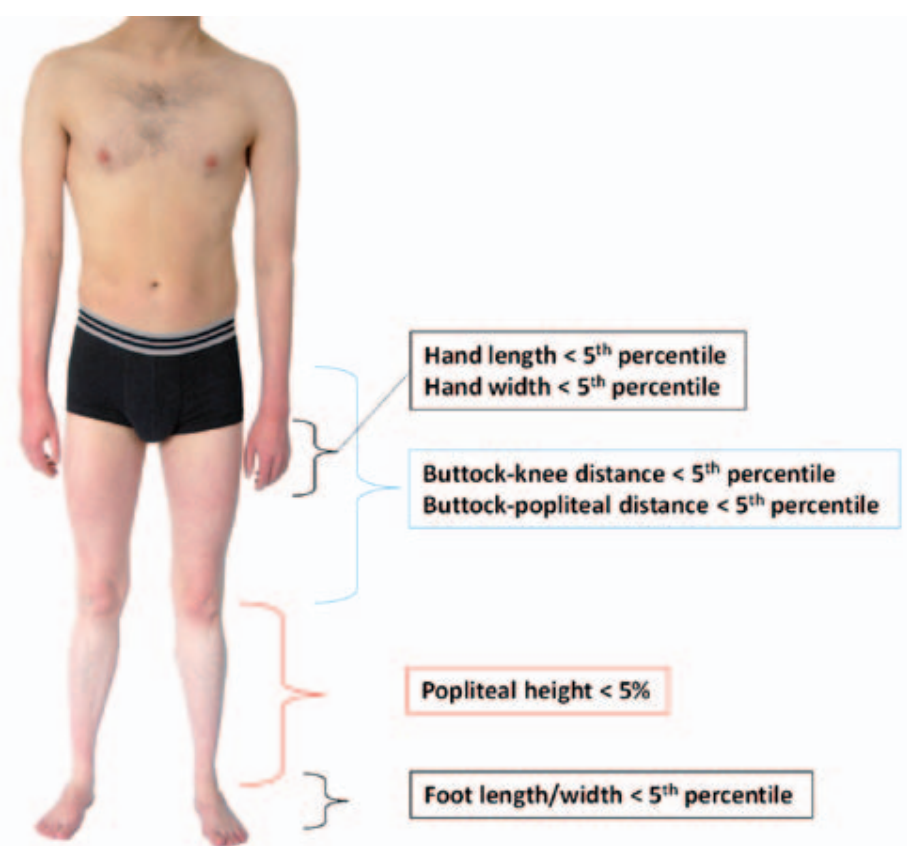

Legend to figure 2. Body habitus of index patient.

Table 4. Quantitative sensory testing (QST), findings and corresponding normative data

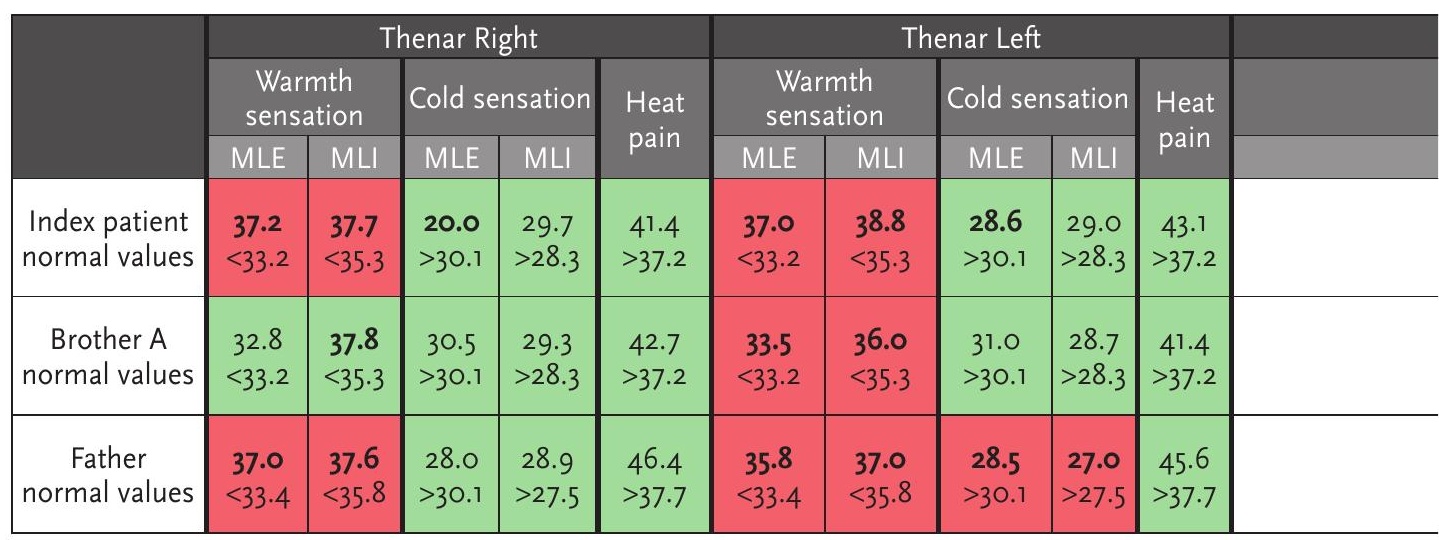

Legend to Table 4. Thresholds were assessed at the dorsum of the foot and thenar eminence bilaterally, using ascending (warm) and descending (cool) thermal energy ramp stimuli delivered through a thermode. Heat pain modality was also examined. Obtained values were compared with reported normative values ${ }^{23}$. A measurement was considered abnormal (bold) when its $Z$ value exceeded 2.5 (see Table for cut-off normative values). A sensory modality was classified as abnormal if the results of both method-of-limits and method-of-levels were abnormal (red). 
Figure 3

Index patient

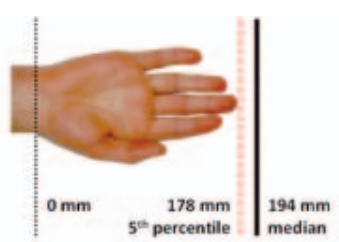

Brother A

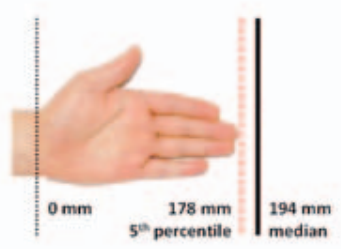

Legend to figure 3. Hands of index patient and family members juxtaposed to age- and gendermatched Dutch community normative values for hand length (indicated by bars to right of hands). The hand and fingers of the father could not be extended completely due to focal arthrosis. Anthropometric findings, and ageand gender-matched normative ranges, are presented in Table 1.

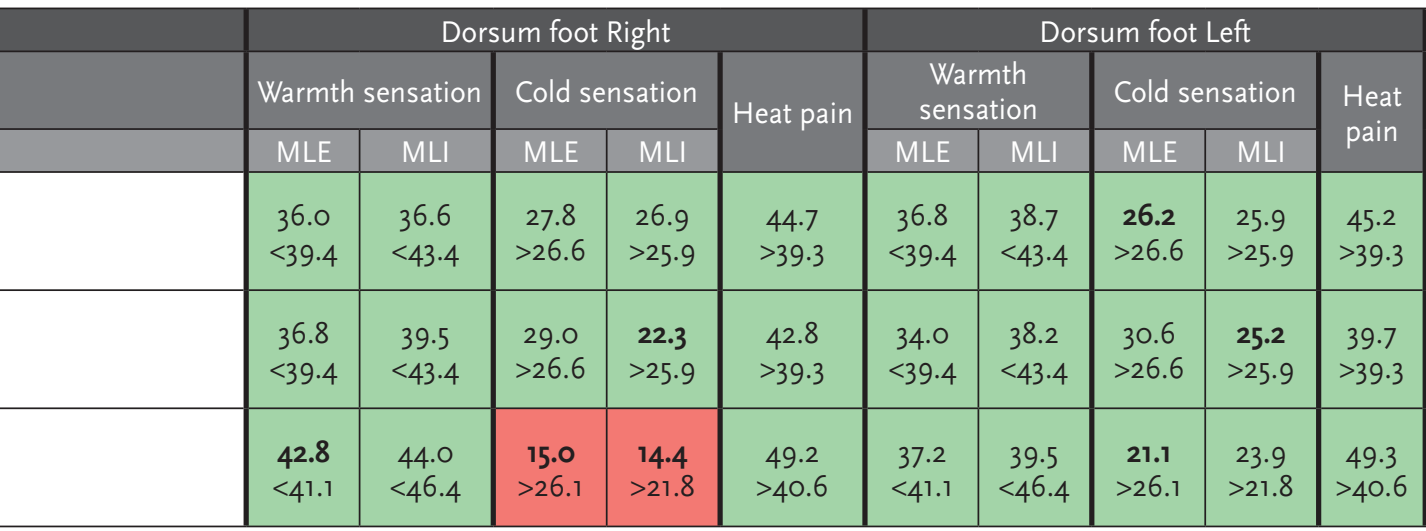




\section{Blood tests}

Blood tests were performed in the index patient, his father and Brother A. The results excluded underlying known causes of small fiber neuropathy, such as impaired glucose tolerance, diabetes mellitus, Fabry's disease, coeliac disease, HIV, sarcoidosis and other systematic illnesses., 4, 24

Quantitative sensory testing and intraepidermal nerve fiber density measurements in the index patient

Quantitative sensory testing showed impaired warmth sensation at the thenar eminence bilaterally (Table 4 ). Intraepidermal nerve fiber density at the lateral malleolus was reduced (intraepidermal nerve fiber density: $5.0 / \mathrm{mm}$ ) compared to age- and gender-matched normative values (median 10.3/ $\mathrm{mm}$, 5 th percentile $\geq 5.2 / \mathrm{mm}),{ }^{16}$ confirming the diagnosis of small fiber neuropathy.

\section{DNA analysis in the index patient}

Sequence analysis ${ }^{6}$ showed a novel mutation (c.2567G>A; G856D) in the $\mathrm{SCN}_{9} \mathrm{~A}$ gene. This mutation substitutes a negatively charged aspartic acid for a highly conserved non-polar glycine within the $\mathrm{S}_{4}-\mathrm{S}_{5}$ linker of domain II of the channel (Fig. 4B). The mutation was not found in a control panel of DNA from 100 healthy Dutch controls (Caucasian) individuals (200 chromosomes), and has not been reported in the NCBI SNP database, the HGMD database, or in the 1000 genomes project.

Findings in proband's family members

The pedigree of this family with G856D mutation is presented in Fig. 4A. Tables 2-4 summarize the findings in the father and Brother $A$ of the index patient, who displayed symptoms that were less severe than in the proband (Tables 2 and 3 ). Quantitative sensory testing was abnormal in both family members (warmth sensation was impaired at the thenar eminence for both; in addition, the father demonstrated impaired cold sensation at the thenar and foot) (Table 4). The intraepidermal nerve fiber density on the other hand was normal. Remarkably, anthropometric data in both the father and brother demonstrated findings quite similar to those in the index patient, with acral and distal limb underdevelopment (Table 1). SCNوA analysis in these two family members with small fiber neuropathy demonstrated the same mutation (c.2567G>A; G856D) as in the index patient (Fig. 4). The unaffected mother and Brother B, without small fiber neuropathy complaints and normal posture, did not harbor the mutation. 
Figure 4.A

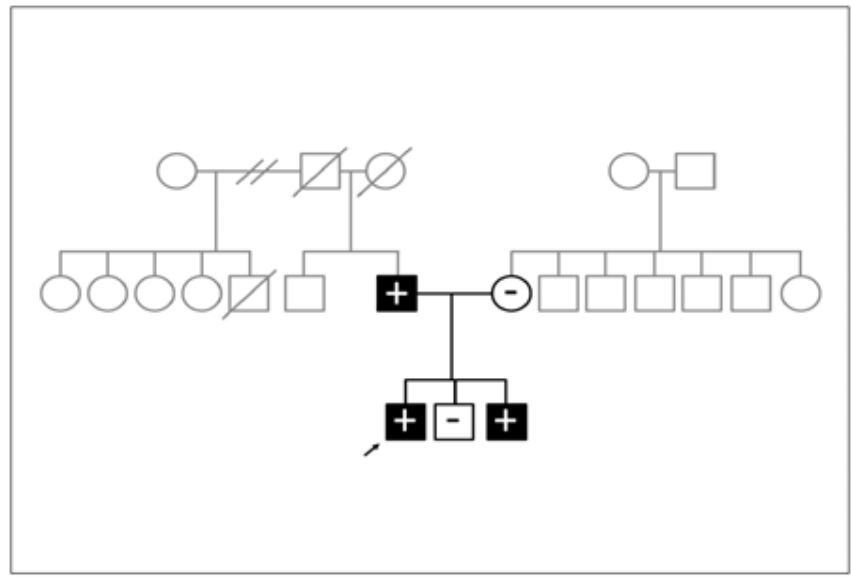

Figure 4.B

Nav1.1

Nav1.2

Nav1.3

Nav1.4

Nav1.5

Nav1.6

Nav1.7

Nav1.8

Nav1.9

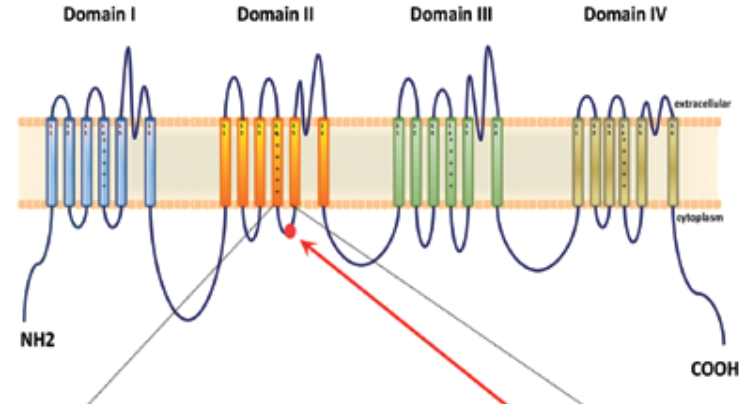

Legend to figure 4. (A) Pedigree showing index patient (arrow) and family members. Family members that underwent $S C N_{9} A$ analysis are depicted in bold. Filled symbols represent affected family members (index patient, father and Brother A) with small fiber neuropathy complaints and novel $S C N 9 A$ mutation $6856 \mathrm{D}$. Open symbols represent the mother and Brother B having no small fiber neuropathy complaints, normal anthropometrics, and no SCNgA mutation (square = male; circle = female; $+=$ G856D mutation present; - $=$ G856D mutation absent). (B) The G856D mutation substitutes an amino acid at a site that is conserved in all mammalian sodium channels (human sodium channels shown). 


\section{Functional analyses}

Functional characterization of $\mathrm{Na}_{\mathrm{v}} \mathrm{T} .7$ mutations

Effects of the mutation on channel properties were assessed by whole-cell voltage clamp after transient transfection of HEK293 cells with wild-type $h \mathrm{Na}_{\mathrm{v}} 1.7_{R}$ or the mutant channel G856D together with $h \beta_{1}$ and $h \beta_{2}$ subunits. Figure ${ }_{5} \mathrm{~A}(1)$ shows representative $\mathrm{Na}_{\mathrm{v}} 1.7$ sodium currents recorded from cells expressing wild-type channels, and Fig. 5A(2) from cells expressing G856D mutant channels. Current density of G856D mutant channel (118 $\pm 13 \mathrm{pA} / \mathrm{pF} ; n$ $=28)$ was significantly smaller $(\mathrm{P}<0.001)$ than that of wild-type $(504 \pm 61 \mathrm{pA} /$ $\mathrm{pF} ; \mathrm{n}=25)$.

The voltage-dependence of channel activation was examined using depolarizing test pulses to $+60 \mathrm{mV}$ from a holding potential of $-120 \mathrm{mV}$. The threshold for activation was $10-15 \mathrm{mV}$ more negative for G856D mutant channels than for wild-type channels (Fig. 5B). The midpoint of activation for G856D (estimated by fitting with a Boltzmann function) was significantly shifted by $-9.3 \mathrm{mV}$ to -32.0 $\pm 0.6 \mathrm{mV}(n=27)$ compared with wild-type channels $(-22.7 \pm 0.8 \mathrm{mV}, \mathrm{n}=23$; $\mathrm{P}<0.001$ ). We measured the kinetics of deactivation, which reflect the transition from the open to the closed state, using single exponential fits to estimate the time of current decay at potentials from -120 to $\pm 40 \mathrm{mV}$ after briefly activating the channels at $-20 \mathrm{mV}$ for $0.5 \mathrm{~ms}$. As shown in Fig. ${ }_{5} \mathrm{C}$, the rates of current decay of G856D mutant channels were significantly slower than those of wild-type channels across all deactivation potentials tested.

Steady-state fast-inactivation was measured using a series of 500-ms prepulses from -150 to omV followed by $40-\mathrm{ms}$ test pulses at $-10 \mathrm{mV}$. The midpoint of fast inactivation for $6856 \mathrm{D}$ mutant channels $(-72.8 \pm 0.8 \mathrm{mV}, \mathrm{n}=27)$ was shifted $6.2 \mathrm{mV}$ in a depolarizing direction compared to that of wild-type channels (-79.0 $\pm 1.0 \mathrm{mV}, \mathrm{n}=22 ; \mathrm{P}<0.001$ ) (Fig. $5 \mathrm{D}$ ). The combination of the depolarizing shift in fast-inactivation and the hyperpolarizing shift in activation results in increased overlap between activation and fast-inactivation, which predicts a large window current.

We measured slow-inactivation with 30-s prepulses, followed by 100-ms pulses to $-120 \mathrm{mV}$ to allow recovery from fast-inactivation, and then a $20-\mathrm{ms}$ test pulse to omV to determine the fraction of available channels. The steady-state slowinactivation curve of G856D mutant channels was shifted in a hyperpolarizating direction (Fig. $5 \mathrm{E})$. The midpoint was $-62.9 \pm 1.3 \mathrm{mV}$ for wild-type $(\mathrm{n}=13)$ and $78.0 \pm 1.1 \mathrm{mV}$ for $\mathrm{G} 856 \mathrm{D}$ mutant channels ( $\mathrm{n}=16$; $\mathrm{P}<0.001$ ).

Recovery from fast-inactivation (repriming) was assessed using pairs of pulses with an incrementally increasing recovery time at six different recovery potentials $(-120,-110,-100,-90,-80$ and $-70 \mathrm{mV})$. G856D mutation did not change the repriming kinetics at any of these six potentials (data not shown). The development of closed-state inactivation was slower for G856D mutant 
channels, and the time constants were significantly longer than those of wildtype channels at $-80,-70$ and $-60 m V$ (Fig. 5 F).

Ramp currents, reflecting the channel's response to the small, slow depolarizations, were evaluated by a slow ramp protocol with a depolarization from -120 to omV over $600 \mathrm{~ms}$. The recorded current was expressed as a percentage of the peak inward current obtained during the activation protocol. For cells expressing wild-type channels, the amplitude of ramp current was $0.73 \pm 0.1 \%(n=10)$. In contrast, for cells expressing G856D mutant channels, the ramp current amplitude was $8.42 \pm 0.49 \%(n=19 ; \mathrm{P}<0.001)$, an 11 -fold increase compared to wild-type. Compared with wild-type channels $(-39.6 \pm 1.3$ $\mathrm{mV}, \mathrm{n}=10$ ), the peak ramp current of G856D mutant channels occurred at more negative potentials $(-50.6 \pm 1.1 \mathrm{mV}, \mathrm{n}=19$; $\mathrm{P}<0.001)$ (Fig. $\left.{ }_{5} \mathrm{G}\right)$, a similar shift as was observed for step depolarization.

We assessed the effect of the mutation on persistent currents, i.e. currents that are not inactivated at the end of 100-ms depolarization pulses, expressed as a percentage of peak current amplitude elicited by step depolarizations. As shown in Fig. $5 \mathrm{H}$ [traces elicited by pulses to $-20 \mathrm{mV}$, from the same cells as shown in Fig. $5 \mathrm{~A}(1)$ and (2)], mutant channels produced markedly larger persistent currents than wild-type channels. The average persistent current amplitude of G856D at -20mV (normalized to peak of transient current) was $3.51 \pm 0.91 \%$ $(n=9)$, >10 times larger than that of wild-type channels $(0.32 \pm 0.11 \%, n=11$; $\mathrm{P}<0.01$ ) (Fig. 5l). Notably, cells expressing G856D mutant channels produced enhanced persistent currents $[1.21 \pm 0.25 \%(n=9)$, compared with $0.13 \pm$ $0.06 \%(n=11)$ for wild-type; $P<0.01]$ even around $-55 \mathrm{mV}$, close to the resting membrane potential of DRG neurons (Fig. $5 \mathrm{I})$. These changes would be expected to increase the excitability of neurons expressing mutant G856D channels.

To assess the effect of the G856D mutation on DRG neuron excitability, we expressed wild-type and G856D mutant channels in small DRG neurons (22-28 $\mathrm{mm}$ diameter) and performed current-clamp recordings. Input resistance was similar between DRG neurons expressing wild-type channels $(1164 \pm 82 \mathrm{M} \Omega$, $n$ = 32) and DRG neurons expressing G856D mutant channels $(1246 \pm 129 \mathrm{M} \Omega$, $\mathrm{n}=21$; P>0.05). However, resting membrane potential of DRG neurons, which expressed G856D mutant channels $(-49.7 \pm 1.1 \mathrm{mV}, \mathrm{n}=21)$ was significantly depolarized by $6.8 \mathrm{mV}$ compared with $\mathrm{DRG}$ neurons expressing wild-type channels $(-56.5 \pm 1.0 \mathrm{mV}, \mathrm{n}=32 ; \mathrm{P}<0.001)$.

Current threshold, the injection stimulus required to produce a single all-ornone action potential, was reduced in DRG neurons expressing G856D. Figure $6 \mathrm{~A}$ shows traces from a representative DRG neuron expressing wild-type channels. In response to $\leq 220 \mathrm{pA}$ sub-threshold current injections, the neuron only generated small, graded membrane potential depolarization. The first all-or-none action potential required a stimulus of $225 \mathrm{pA}$ (current threshold 
for this neuron). Figure $6 \mathrm{~B}$ shows recordings from a representative $\mathrm{DRG}$ neuron, which expressed G856D mutant channels. For this neuron, the current injection required to produce the first all-or-none action was $100 \mathrm{pA}$. Figure 6C presents a comparison of current threshold between these two groups of neurons. Expression of G856D (109 $\pm 16 \mathrm{pA}, \mathrm{n}=21)$ reduced current threshold significantly compared with wild-type $\mathrm{Na}_{\mathrm{v}} 1.7(222 \pm 19 \mathrm{pA}, \mathrm{n}=32 ; \mathrm{P}<0.001)$. There were no significant differences of either voltage threshold (the voltage at which action potential take-off occurs) (wild-type: -21.2 $\pm 1.2 \mathrm{mV}, \mathrm{n}=32$; G856D: $-23.9 \pm 1.3 \mathrm{mV}, \mathrm{n}=21 ; \mathrm{P}>0.05$ ) or action potential amplitude (wild-type: 107.1 $\pm 2.3 \mathrm{mV}, \mathrm{n}=32$; G856D:104.4 $\pm 2.0 \mathrm{mV}, \mathrm{n}=21 ; \mathrm{P}>0.05$ ) between these two groups of DRG neurons.

To evaluate repetitive action potential firing, we injected DRG neurons with a series of 500-ms current stimuli from 25 to 500 pA in 25 pA increments. DRG neurons expressing G856D mutant channels fired more action potentials compared with neurons expressing wild-type channels at all stimulus levels. Figure 6D-I show the responses of two representative DRG neurons that expressed wild-type and G856D mutant channels, respectively, to 500-ms current steps at $1 x, 2 x$ and $3 x$ current thresholds for the respective neuron. DRC neurons expressing wild-type channels generated a single spike in response to current injections at both 1 - and 2-fold current threshold, and generated two spikes in response to stimuli at 3 -fold current threshold. In contrast, neurons expressing G856D mutant channels tended to fire with multiple action potentials in response to stimuli at 2- and 3-fold threshold. Figure 6/ compares the responses of DRG neurons expressing G856D mutant channels and wildtype $\mathrm{Na}_{\mathrm{v}} 1.7$ channels over a range of graded suprathreshold stimulation levels. We also found that G856D mutation produced an increase in the proportion of spontaneously firing DRG neurons. Figure $6 \mathrm{~K}$ shows an example of sustained spontaneous firing from a representative DRG neuron expressing G856D mutant channels. Figure $6 \mathrm{~L}$ displays $2 \mathrm{~s}$ of spontaneous firing from this neuron on an expanded time-base. As the bar graph in Fig. $6 \mathrm{~L}$ shows, only $3 \%(1 / 33)$ of DRG neurons expressing wild-type channels displayed spontaneous firing. In contrast, $36 \%$ of DRG neurons expressing G856D displayed spontaneous firing (12/33 cells, $36.4 \%$ ), significantly more than for wild-types ( $P<0.05$, z-test).

Taken together, the current-clamp results show that the G856D mutation depolarizes resting membrane potential of DRG neurons, increases excitability of these cells as evidenced by reduced current threshold, increases firing rates in response to suprathreshold stimulation and increases the proportion of cells that fire spontaneously in the absence of stimulation. 
Figure 5.
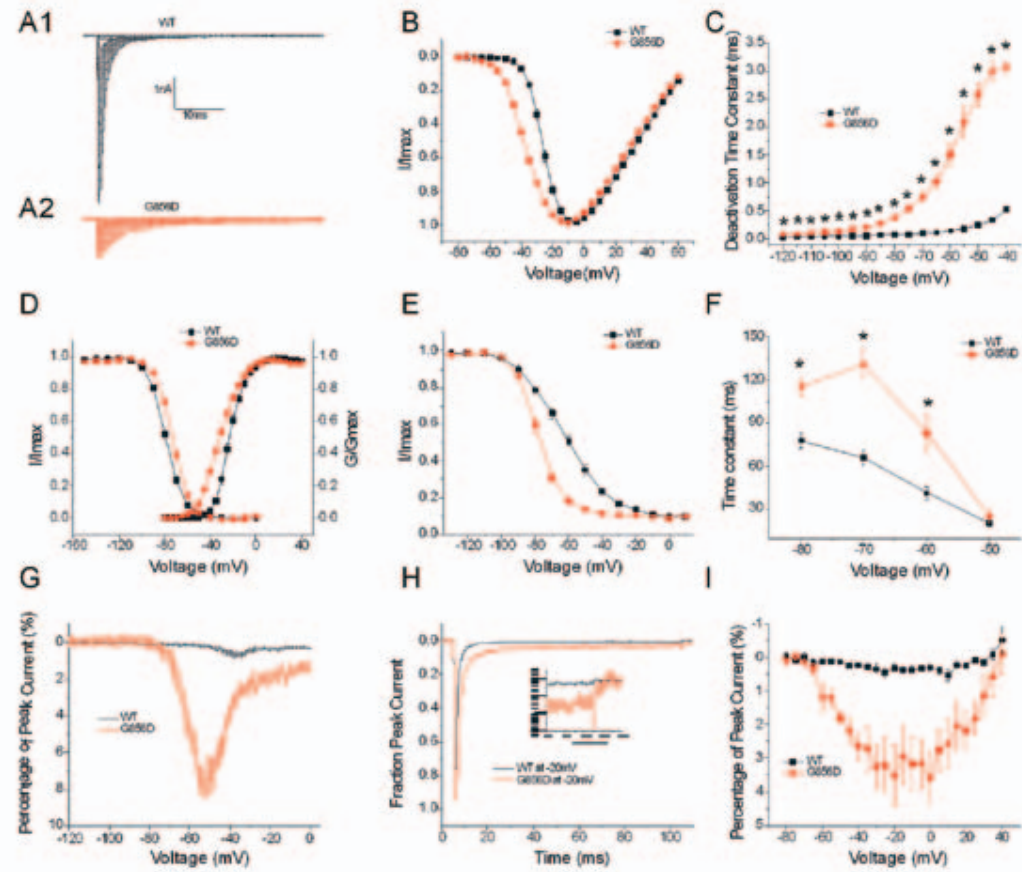

Legend to figure 5. Voltage-clamp analysis of $\mathrm{Na}_{\mathrm{v}} 1.7 / \mathrm{G} 856 \mathrm{D}$ mutant channels. (A) Representative current traces recorded from HEK293 cells expressing wild-type (WT) ( $A_{1}$ ) or G856D (A2) channels, evoked by voltage steps $(100 \mathrm{~ms})$ from -80 to $60 \mathrm{mV}$ in $5 \mathrm{mV}$ increments, from a holding potential of -120 mV. (B) Normalized peak current-voltage relationship curves for wild-type (black squares, $n=23$ ) and G856D (red circles, $n=27$ ) channels. G856D shifts threshold for channel activation by $10-15 \mathrm{mV}$ in a hyperpolarizing direction. (C) Time constants for tail current deactivation. Cells were held at $-120 \mathrm{mV}$ and tail currents were generated by a brief $0.5-\mathrm{ms}$ depolarization to $-20 \mathrm{mV}$ followed by a series of repolarizations ranging from -120 to $-40 \mathrm{mV}, * \mathrm{P}<0.05$. G856D channels (red circles, $n=19$ ) deactivate significantly slower than wild-type channels (black squares, $n$ $=13$ ). (D) Steady-state fast inactivation for wild-type (black squares, $n=22$ ) and G856D (red circles, $n=27$ ) channels, examined using a series of 500-ms prepulses from -150 to omV followed by test pulses to $-10 \mathrm{mV}$. G856D mutation shifts $\mathrm{V}_{1 / 2}$ for fast-inactivation by $6.2 \mathrm{mV}$ in a depolarized direction. The voltage-dependence of activation was obtained by converting current to conductance, and fitting Boltzmann functions to data shown in B. G856D mutation shifts $V_{1 / 2}$ of activation by $-9.3 \mathrm{mV}$. (E) Steady-state slow-inactivation curves for wild-type (black squares, $\mathrm{n}=$ 13) and G856D (red circles, $n=16$ ), assessed using a 20-ms pulse to -10mV after a 30-s prepulse to potentials ranging from -130 to $10 \mathrm{mV}$ followed by a $100-\mathrm{ms}$ pulse to $-120 \mathrm{mV}$ to remove fastinactivation. G856D mutation enhances steady-state slow-inactivation with the $V_{1 / 2}$ shifting by $-15.1 \mathrm{mV}$. (F) Time constants for development of closed-state inactivation, measured by holding at $-120 \mathrm{mV}$, prepulsing membrane potential to the inactivation potential for increasing durations, then stepping to $-10 \mathrm{mV}$ to determine the fraction of current inactivated during the prepulse. Data were fit with single exponential. G856D mutation (red circles) slows the development of closedstate inactivation significantly compared with wild-type (black squares) from -80 to $-60 \mathrm{mV}$. (G) Representative ramp currents, elicited with 600-ms ramp depolarization from -120 to omV for wild-type (black) and G856D mutant (red). G856D mutation increases ramp current amplitude and shifts the voltage at which the peak of ramp current occurs. $(H)$ The traces elicited by pulses 
to $-20 \mathrm{mV}$ (the same two cells as in A), and the currents were normalized to peak inward currents. G856D-expressing (red line) cell produces larger persistent current than wild-type-expressing (black line) cell. Inset: The end of the 100-ms trace and vertical axis were expanded to show the difference in persistent current between wild-type and G856D mutant channels at the end of 100ms recording. (I) Comparison of persistent currents between cells expressing wild-type channels $(n=11)$ and cells expressing G856D mutant channels $(n=9)$ for activation depolarization step pulses from -80 to $40 \mathrm{mV}$.

Figure 6.

A

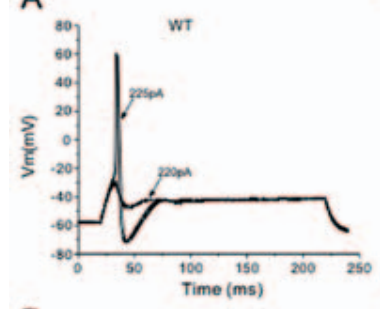

D

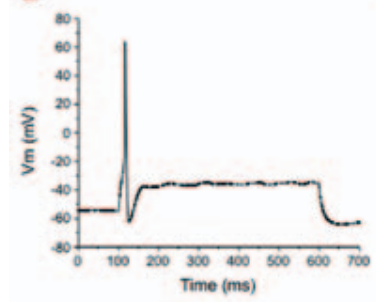

G

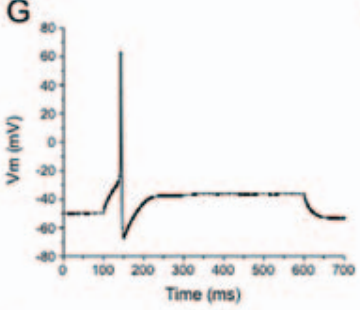

$J$

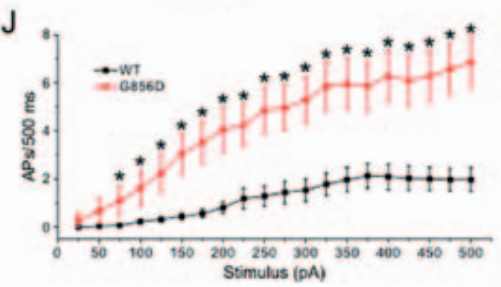

B

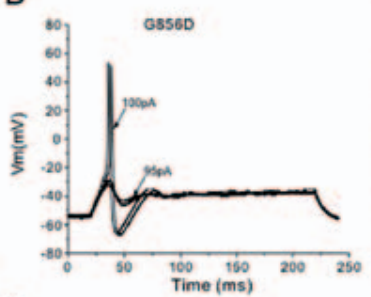

$\mathrm{E}$

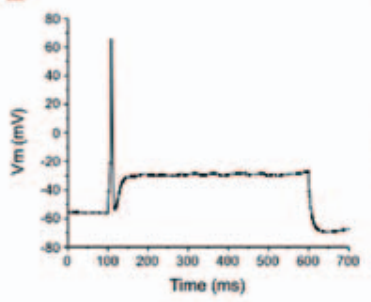

$\mathrm{H}$

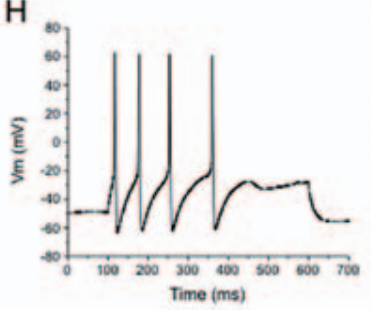

K

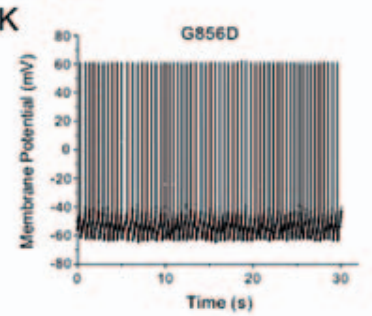

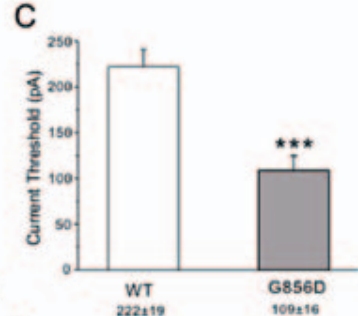

$\mathrm{F}$

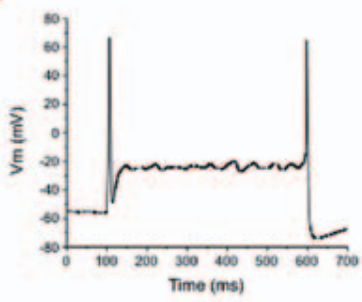

I

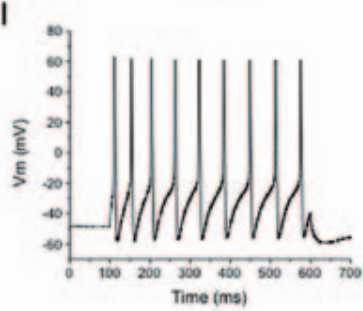

L

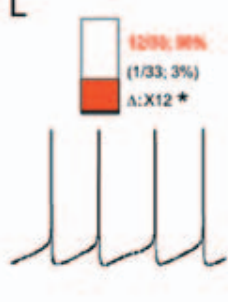

Legend to figure 6. Current-clamp analysis of effects of C856D mutant channels on DRG neuron excitability. ( $A$ and $B$ ) Representative action potential (AP) traces recorded from DRG neuron expressing wild-type (WT) (A) or G856D mutant (B) channels. Action potentials were elicited by 
200-ms step depolarizing current injections from resting membrane potential. (C) Comparison of current threshold for DRG neurons expressing wild-type and G856D mutant channels. Expression of G856D channels reduces current threshold significantly. Current threshold: wild-type: $222 \pm 19$ $\mathrm{pA}, \mathrm{n}=32$; G856D: $109 \pm 16 \mathrm{pA}, \mathrm{n}=21$. $* \mathrm{w}^{\prime} *$ P50.001. (D-F) Responses of a representative DRG neuron expressing wild-type channels to 500-ms depolarization current steps that are $1 x(D), 2 x$ $(\mathrm{E})$ and $3 \times(F)$ the current threshold of this neuron. $(\mathrm{G}-\mathrm{I})$ Responses of a representative DRG neuron expressing G856D mutant channels to 500-ms depolarization current steps that are $1 \mathrm{x}$ $(\mathrm{G}), 2 \mathrm{x}(\mathrm{H})$ and $3 \times(\mathrm{I})$ the current threshold of this neuron. $(\mathrm{J})$ Comparison of responses (number of impulses evoked by a 500-ms stimulus) in DRG neurons expressing wild-type (black square, $n=32$ ) and G856D channels (red circle, $n=21$ ) across a range of step current injections from $25-500 \mathrm{pA}, * \mathrm{P}<0.05$. (K) representative recording showing spontaneous firing of DRG neuron expressing G856D mutant channels. Trace was recorded for $30 \mathrm{~s}$ without any current injection. (L) Top: Bar graph showing the proportion of spontaneously firing DRG neurons expressing G856D (red) and wild-type channels (black); numbers to the right of the bar graph show values for wildtype (black font) and G856D (orange font); $\mathrm{x} 12$ indicates 12 -fold increase; $* \mathrm{P}<0.05$. Bottom: $2 \mathrm{~s}$ of spontaneous firing for the same neuron as in (K), shown on an expanded time-base.

\section{Discussion}

The $\mathrm{Na}_{\mathrm{v}} 1.7$ sodium channel is abundantly expressed within DRG and sympathetic ganglion neurons $\mathrm{s}^{7,8}$ and in their small-diameter axons within peripheral nerves. ${ }^{9}$ Single amino acid substitutions of $\mathrm{Na}_{\mathrm{v}} 1.7$ that produce gain-of-function have been associated with three classes of disorders: (i) $\mathrm{Na}_{\mathrm{v}} 1.7$ mutations that enhance activation have been shown to produce inherited erythromelalgia; ${ }^{20}$ ${ }_{21,25-27}$ (ii) mutations of $\mathrm{Na}_{\mathrm{v}} 1.7$ that impair fast-inactivation produce paroxysmal extreme pain disorder; ${ }^{28}$ and (iii) single amino acid substitutions of $\mathrm{Na}_{\mathrm{v}} 1.7$ that impair slow-inactivation, impair fast- and slow-inactivation, or enhance resurgent currents have been found in $\sim 30 \%$ of patients with idiopathic small fiber neuropathy. ${ }^{6}$

In the present article, we describe a novel syndrome of painful small fiber neuropathy, small hands and small feet, in a family housing a $\mathrm{Na}_{\mathrm{v}} \mathrm{l} .7$ mutation that enhances activation, impairs fast-inactivation and markedly enhances persistent current and the response to slow ramp stimuli. The patient described here complained of burning pain and redness of the hands and feet, triggered by warmth and relieved by cold, similar to inherited erythromelalgia ${ }^{26}$ and to one of the previously described patients with idiopathic small fiber neuropathy associated with a $\mathrm{Na}_{\mathrm{v}} \mathrm{r} .7$ variant. ${ }^{6}$ However, unlike most patients with inherited erythromelalgia, ${ }^{18}$ the present patient also displayed profound autonomic dysfunction, a common clinical feature of small fiber neuropathy. ${ }^{1-4}$ Demonstration of decreased intraepidermal nerve fiber density on skin biopsy and abnormal quantitative sensory testing confirmed the diagnosis of small fiber neuropathy in the index patient, who also displayed under-development of the hands and feet. Intraepidermal nerve fiber density was normal in the 
proband's father and brother; however, they also displayed distal extremity pain and redness, met diagnostic criteria for small fiber neuropathy on quantitative sensory testing and had small distal extremities (acromesomelia). Our analysis of $S C N g A$ in these three patients and two unaffected family members revealed a novel $\mathrm{Na}_{\mathrm{v}} 1.7$ (G856D) mutation that increases DRG neuron excitability, which segregated with disease.

The G856D mutation substitutes a negatively charged aspartic acid for a highly conserved non-polar glycine within the $\mathrm{S}_{4}-\mathrm{S}_{5}$ linker of channel domain II, close to the sites of several mutations that cause inherited erythromelalgia. ${ }^{25,27}$ Like most inherited erythromelalgia mutations that have been described to date, ${ }^{26}$ the G856D mutation hyperpolarizes the voltage dependence of activation, slows deactivation and enhances the channel's response to slow ramp stimuli. Current-clamp analysis demonstrates that G856D, like other $\mathrm{Na}_{\mathrm{v}} 1.7$ gain-offunction mutations, increases the excitability of DRG neurons, a change that underlies pain. ${ }^{26}$ In contrast to inherited erythromelalgia mutations, and to $\mathrm{Na}_{\mathrm{v}} 1.7$ variants previously reported in small fiber neuropathy, ${ }^{6,26}$ the G856D mutation produced a dramatic increase in persistent current (11-fold at -20mV and 9 -fold at $\pm 55 \mathrm{mV}$, close to the resting potential of DRG neurons), a change that would be expected to increase excitability of DRG neurons. ${ }^{29,30}$

The patients described in this article are notable in displaying acromesomelia, or under-development of distal hands and feet. ${ }^{31}$ Limb morphogenesis is a complex process involving multiple signalling cascades that have been partially explicated at the molecular and genetic levels. Several gene mutations have been associated with syndromal and non-syndromal limb defects. ${ }^{32-35}$ The role of sodium channels in limb development is not understood, and no relationship between mutations in sodium channels and small limbs has previously been described.

$\mathrm{Na}_{\mathrm{v}} 1.7$ is co-expressed with $\mathrm{Na}_{\mathrm{v}} 1.6, \mathrm{Na}_{\mathrm{v}} 1.8$ and $\mathrm{Na}_{\mathrm{v}} 1.9$ and the sodium-calcium exchanger $\mathrm{NCX}_{2}$ in small-diameter DRG neurons and their distal terminals, ${ }^{9}$ where a high surface-to-volume ratio and high input impedance are expected to enhance the effect of sodium channel activity. ${ }^{6,37}$ In non-myelinated axons, the energetic cost of action potential conduction per impulse per unit length is $\sim 10$-fold higher than in larger myelinated axons..$^{38}$ Sodium channel blockers have been shown to have a protective effect, limiting the degree of axonal degeneration, in multiple axon injury model. ${ }^{39-43}$ It remains to be determined whether the hyperexcitability produced in DRG neurons by $\mathrm{G} 856 \mathrm{D}$ poises small axons to degenerate, or whether the robust persistent current of G856D mutant channels can trigger reverse sodium-calcium exchange, which injures smalldiameter axons, as has been demonstrated in CNS white matter. ${ }^{44,45}$

Anthropometric assessments have not been carried out in individuals harboring $\mathrm{Na}_{\mathrm{v}}$ 1.7 mutations that substitute other amino acids within the domain II S4- 
$\mathrm{S}_{5}$ linker, ${ }^{25,27,30}$ close to $\mathrm{G} 856$, or more generally in patients harboring $\mathrm{Na}_{\mathrm{v}} 1.7$ variants. ${ }^{6,}{ }^{46}$ While we cannot rule out the possibility that acromesomelia in the three affected individuals is the result of a second mutation or chance association, the segregation of the G856D mutation with acromesomelia, and the similar topographic pattern of distal limb pain and redness and distal limb under-development in this kindred suggest that the G856D mutation may possibly contribute to acromesomelia.

Although $\mathrm{Na}_{\mathrm{v}} \mathrm{T} .7$ has not been reported in bone or cartilage cells at any stage of development, $\mathrm{Na}_{\mathrm{v}} \mathrm{T} .2$ sodium channels are expressed in osteoblasts during embryogenesis, ${ }^{47}$ and we cannot exclude an effect of the G856D mutation on limb development due to transient expression of $\mathrm{Na}_{\mathrm{v}} 1.7$ within non-neuronal cells at some stage of ontogenesis. Alternatively, our observations of small nerve fiber loss in the index patient, and of abnormal function of small-diameter axons innervating the limb as evidenced by the clinical picture and abnormal quantitative sensory testing in all three affected patients in the context of a sodium channel mutation that markedly enhances persistent current and DRG neuron excitability, suggest the possibility that dysfunction or injury of peripheral nerve fibers may have interfered with normal limb development.

Impaired limb growth has been reported following denervation (brachial plexus neurectomy) in newborn rabbits. ${ }^{8}$ Reddening of the skin in the present patient might be interpreted as suggesting altered vasomotor function, possibly due to an effect of the mutation on sympathetic ganglion neurons that express $\mathrm{Na}_{\mathrm{v}} 1.7 .{ }^{8}$ Innervation of bone vasculature ${ }^{49-52}$ raises the possibility that altered vascular perfusion may have affected limb morphogenesis during development. Bones are innervated by small myelinated and unmyelinated sensory and sympathetic nerves, ${ }^{49-51,53-58}$ some of which contain peptides such as calcitonin gene-related peptide. ${ }^{57,58}$ Calcitonin gene-related peptide has been implicated as a modulator of the metabolism of bone cells, since it regulates osteoclast and osteoblast function, thus modulating bone formation and resorption. ${ }^{51,52,54.58}$ In addition, nerve fibers are frequently located in the epiphyseal region, a location that has been interpreted as suggesting that they may participate in the regulation of the epiphyseal growth plate. ${ }^{51,55}$

Several disorders are known to cause both neuropathy and limb defects in the embryo. An example is the teratogenic effect of thalidomide, a sedative and hypnotic drug formerly used as an anti-emetic in pregnancy, which has been linked to severe limb deformities in children, especially bone deformities such as phocomelia.59 Several models have been postulated to explain the limb teratogenesis produced by thalidomide. One model suggests a direct effect on sensory neurons or neural crest, which may produce an embryonic peripheral neuropathy resulting in impaired trophic function of the nerves with consequent under-development of the limbs. ${ }^{60-62}$ Another example is the teratogenicity of 
maternal varicella-zoster infection, which may lead to a peripheral neuropathy that is associated with hypoplastic limbs. ${ }^{63}$ A similar mechanism has been suggested in congenital malformations in infants of diabetic mothers. ${ }^{64,65}$ Taken together with these earlier studies, the coexistence of small fiber neuropathy and acromesomelia in this kindred with a gain-of-function $\mathrm{Na}_{\mathrm{v}} 1.7$ mutation leads us to speculate that dysfunction of small nerve fibers innervating the limbs may have adversely affected limb morphogenesis at some stage during development.

In conclusion, we describe a novel syndrome of distal pain, dysautonomia, small hands and small feet in a kindred with a novel missense SCNgA mutation that causes multiple gain-of-function changes in $\mathrm{Na}_{\mathrm{v}} \mathrm{1} .7$, including markedly enhanced persistent current. Future studies will be needed to test the hypothesis that peripheral nerve dysfunction due to this mutation can contribute to impaired limb development. 


\section{References}

1. Gorson, K.C. \& Ropper, A.H. Idiopathic distal small fiber neuropathy. Acta Neurol Scand 92, 376-82 (1995).

2. Holland, N.R. et al. Small-fiber sensory neuropathies: clinical course and neuropathology of idiopathic cases. Ann Neurol 44, 47-59 (1998).

3. Lacomis, D. Small-fiber neuropathy. Muscle Nerve 26, 173-88 (2002).

4. Devigili, G. et al. The diagnostic criteria for small fibre neuropathy: from symptoms to neuropathology. Brain 131, 1912-25 (2008).

5. Tesfaye, S. et al. Diabetic neuropathies: update on definitions, diagnostic criteria, estimation of severity, and treatments. Diabetes Care 33, 2285-93 (2010).

6. Faber, C.G. et al. Gain of function $\mathrm{Na}(\mathrm{V}) 1.7$ mutations in idiopathic small fiber neuropathy. Ann Neurol 71, 26-39 (2012).

7. Toledo-Aral, J.). et al. Identification of $\mathrm{PN}$, a predominant voltage-dependent sodium channel expressed principally in peripheral neurons. Proc Natl Acad Sci U S A 94, 1527-32 (1997).

8. Rush, A.M. et al. A single sodium channel mutation produces hyper- or hypoexcitability in different types of neurons. Proc Natl Acad Sci U S A 103, 8245-50 (2006).

9. Persson, A.K. et al. Sodium-calcium exchanger and multiple sodium channel isoforms in intra-epidermal nerve terminals. Mol Pain 6, 84 (2010).

10. Herzog, R.I., Cummins, T.R., Ghassemi, F., Dib-Hajj, S.D. \& Waxman, S.G. Distinct repriming and closedstate inactivation kinetics of Nav1.6 and Nav1.7 sodium channels in mouse spinal sensory neurons. J Physiol 551, 741-50 (2003).

11. Steenbekkers, L.P.A. \& van Beijsterveldt, C.E.M. (eds.) Design Relevant characteristics of ageing users. (Delft University Press, TU Delft, Delft, 1998).

12. Molenbroek, J.F. (2004).

13. Hoitsma, E. et al. Abnormal warm and cold sensation thresholds suggestive of small-fibre neuropathy in sarcoidosis. Clin Neurophysiol 114, 2326-33 (2003).

14. Reulen, J.P., Lansbergen, M.D., Verstraete, E. \& Spaans, F. Comparison of thermal threshold tests to assess small nerve fiber function: limits vs. levels. Clin Neurophysiol 114, 556-63 (2003).

15. Shy, M.E. et al. Quantitative sensory testing: report of the Therapeutics and Technology Assessment Subcommittee of the American Academy of Neurology. Neurology 60, 898-904 (2003).

16. Lauria, G. et al. European Federation of Neurological Societies/Peripheral Nerve Society Guideline on the use of skin biopsy in the diagnosis of small fiber neuropathy. Report of a joint task force of the European Federation of Neurological Societies and the Peripheral Nerve Society. Eur J Neurol 17, 903-12, e44-9 (2010).

17. Lauria, G. et al. Intraepidermal nerve fiber density at the distal leg: a worldwide normative reference study. J Peripher Nerv Syst 15, 202-7 (2010).

18. Drenth, J.P. et al. SCNgA mutations define primary erythermalgia as a neuropathic disorder of voltage gated sodium channels. J Invest Dermatol 124, 1333-8 (2005).

19. Klugbauer, N., Lacinova, L., Flockerzi, V. \& Hofmann, F. Structure and functional expression of a new member of the tetrodotoxin-sensitive voltage-activated sodium channel family from human neuroendocrine cells. EMBOJ 14, 1084-90 (1995).

20. Choi, J.S. et al. Alternative splicing may contribute to time-dependent manifestation of inherited erythromelalgia. Brain 133, 1823-35 (2010).

21. Dib-Hajj, S.D. et al. Gain-of-function mutation in Nav1.7 in familial erythromelalgia induces bursting of sensory neurons. Brain 128, 1847-54 (2005).

22. Galer, B.S. \& Jensen, M.P. Development and preliminary validation of a pain measure specific to neuropathic pain: the Neuropathic Pain Scale. Neurology 48, 332-8 (1997).

23. Yarnitsky, D. \& Sprecher, E. Thermal testing: normative data and repeatability for various test algorithms. J Neurol Sci 125, 39-45 (1994).

24. Lauria, G. Small fibre neuropathies. Curr Opin Neurol 18, 591-7 (2005).

25. Cummins, T.R., Dib-Hajj, S.D. \& Waxman, S.G. Electrophysiological properties of mutant Nav1.7 sodium channels in a painful inherited neuropathy. J Neurosci 24, 8232-6 (2004).

26. Dib-Hajj, S.D., Cummins, T.R., Black, J.A. \& Waxman, S.G. Sodium channels in normal and pathological pain. Annu Rev Neurosci 33, 325-47 (2010). 
27. Han, C. et al. Sporadic onset of erythermalgia: a gain-of-function mutation in Nav1.7. Ann Neuro/ 59, 553-8 (2006).

28. Fertleman, C.R. et al. SCNgA mutations in paroxysmal extreme pain disorder: allelic variants underlie distinct channel defects and phenotypes. Neuron 52, 767-74 (2006).

29. Dib-Hajj, S.D. et al. Paroxysmal extreme pain disorder M1627K mutation in human Nav1.7 renders DRG neurons hyperexcitable. Mol Pain 4, 37 (2008).

30. Estacion, M. et al. NaV1.7 gain-of-function mutations as a continuum: A $1632 \mathrm{E}$ displays physiological changes associated with erythromelalgia and paroxysmal extreme pain disorder mutations and produces symptoms of both disorders. J Neurosci 28, 11079-88 (2008).

31. Langer, L.O. \& Garrett, R.T. Acromesomelic dysplasia. Radiology 137, 349-55 (1980).

32. Gurrieri, F., Kjaer, K.W., Sangiorgi, E. \& Neri, G. Limb anomalies: Developmental and evolutionary aspects. Am J Med Genet 115, 231-44 (2002).

33. Barham, G. \& Clarke, N.M. Genetic regulation of embryological limb development with relation to congenital limb deformity in humans. J Child Orthop 2, 1-9 (2008).

34. Lyons, K. \& Ezaki, M. Molecular regulation of limb growth. J Bone Joint Surg Am 91 Suppl 4, 47-52 (2009).

35. Decker, E. et al. FGFR3 is a target of the homeobox transcription factor SHOX in limb development. Hum Mol Genet 20, 1524-35 (2011).

36. Waxman, S.G., Black, J.A., Kocsis, J.D. \& Ritchie, J.M. Low density of sodium channels supports action potential conduction in axons of neonatal rat optic nerve. Proc Natl Acad Sci U S A 86, 1406-10 (1989).

37. Donnelly, D.F. Spontaneous action potential generation due to persistent sodium channel currents in simulated carotid body afferent fibers. J Appl Physiol 104, 1394-401 (2008).

38. Wang, S.S. et al. Functional trade-offs in white matter axonal scaling. J Neurosci 28, 4047-56 (2008).

39. Stys, P.K., Ransom, B.R. \& Waxman, S.G. Tertiary and quaternary local anesthetics protect CNS white matter from anoxic injury at concentrations that do not block excitability. J Neurophysiol 67, 236-40 (1992).

40. Kapoor, R., Davies, M., Blaker, P.A., Hall, S.M. \& Smith, K.J. Blockers of sodium and calcium entry protect axons from nitric oxide-mediated degeneration. Ann Neurol 53, 174-80 (2003).

41. Lo, A.C., Saab, C.Y., Black, J.A. \& Waxman, S.G. Phenytoin protects spinal cord axons and preserves axonal conduction and neurological function in a model of neuroinflammation in vivo. J Neurophysiol 90 , 3566-71 (2003).

42. Bechtold, D.A., Kapoor, R. \& Smith, K.J. Axonal protection using flecainide in experimental autoimmune encephalomyelitis. Ann Neurol 55, 607-16 (2004)

43. Bechtold, D.A. et al. Axonal protection in experimental autoimmune neuritis by the sodium channel blocking agent flecainide. Brain 128, 18-28 (2005).

44. Stys, P.K., Waxman, S.G. \& Ransom, B.R. Ionic mechanisms of anoxic injury in mammalian CNS white matter: role of $\mathrm{Na}+$ channels and $\mathrm{Na}(+)-\mathrm{Ca}+$ exchanger. J Neurosci 12, 430-9 (1992).

45. Stys, P.K., Sontheimer, H., Ransom, B.R. \& Waxman, S.G. Noninactivating, tetrodotoxin-sensitive Na+ conductance in rat optic nerve axons. Proc Natl Acad Sci U S A 90, 6976-80 (1993).

46. Dib-Hajj, S.D. \& Waxman, S.G. Isoform-specific and pan-channel partners regulate trafficking and plasma membrane stability; and alter sodium channel gating properties. Neurosci Lett 486, 84-91 (2010).

47. Black, J.A., Westenbroek, R.E., Catterall, W.A. \& Waxman, S.G. Type II brain sodium channel expression in non-neuronal cells: embryonic rat osteoblasts. Brain Res Mol Brain Res 34, $89-98$ (1995).

48. Alharby, S.W. Anterior cruciate ligament injuries in growing skeleton. Int J Health Sci (Qassim) 4, 71-9 (2010).

49. Hurrell, D.J. The Nerve Supply of Bone. J Anat 72, 54-61 (1937).

50. Sherman, M. Sarcomas of bone. Med Times 91, 229-42 (1963).

51. Bjurholm, A., Kreicbergs, A., Brodin, E. \& Schultzberg, M. Substance P- and CGRP-immunoreactive nerves in bone. Peptides 9, 165-71 (1988).

52. Hukkanen, M. et al. Rapid proliferation of calcitonin gene-related peptide-immunoreactive nerves during healing of rat tibial fracture suggests neural involvement in bone growth and remodelling. Neuroscience 54, 969-79 (1993).

53. Cooper, R.R. Nerves in cortical bone. Science 160, 327-8 (1968).

54. Hohmann, E.L., Elde, R.P., Rysavy, J.A., Einzig, S. \& Gebhard, R.L. Innervation of periosteum and bone by sympathetic vasoactive intestinal peptide-containing nerve fibers. Science 232, 868-71 (1986). 
55. Hill, E.L. \& Elde, R. Distribution of CGRP-, VIP-, D beta H-, SP-, and NPY-immunoreactive nerves in the periosteum of the rat. Cell Tissue Res 264, 469-80 (1991).

56. Mach, D.B. et al. Origins of skeletal pain: sensory and sympathetic innervation of the mouse femur. Neuroscience 113, 155-66 (2002).

57. Jimenez-Andrade, J.M. et al. A phenotypically restricted set of primary afferent nerve fibers innervate the bone versus skin: therapeutic opportunity for treating skeletal pain. Bone 46, 306-13 (2010).

58. Castaneda-Corral, G. et al. The majority of myelinated and unmyelinated sensory nerve fibers that innervate bone express the tropomyosin receptor kinase A. Neuroscience 178, 196-207 (2011).

59. Vargesson, N. Thalidomide-induced limb defects: resolving a 50-year-old puzzle. Bioessays 31, 1327-36 (2009).

6o. McCredie, J. \& McBride, W.G. Some congenital abnormalities: possibly due to embryonic peripheral neuropathy. Clin Radiol 24, 204-11 (1973).

61. McCredie, J. \& McLeod, J.G. Letter: Thalidomide and embryonic neuropathy. Lancet 1, 111 (1974).

62. McCredie, J., North, K. \& de longh, R. Thalidomide deformities and their nerve supply. J Anat 139 ( Pt 3), 397-410 (1984).

63. Savage, M.O., Moosa, A. \& Gordon, R.R. Maternal varicella infection as a cause of fetal malformations. Lancet 1, 352-4 (1973).

64. Rajbhandari, S.M., Jenkins, R.C., Davies, C. \& Tesfaye, S. Charcot neuroarthropathy in diabetes mellitus. Diabetologia 45, 1085-96 (2002).

65. Botek, G., Anderson, M.A. \& Taylor, R. Charcot neuroarthropathy: An often overlooked complication of diabetes. Cleve Clin J Med 77, 593-9 (2010). 



\section{Chapter 8}

\section{Genetic aspects of sodium channelopathy in small}

fiber neuropathy

J.G.J. Hoeijmakers, I.S.J. Merkies ${ }^{a, b}$, M.M. Gerrits, ${ }^{c}$ S.G. Waxman ${ }^{d, e}$, Faber CG ${ }^{a}$.

aDepartment of Neurology, Maastricht University Medical Center, Maastricht bDepartment of Neurology, Spaarne Hospital, Hoofddorp 'Department of Clinical Genomics, Maastricht University Medical Center, Maastricht ${ }^{d}$ Department of Neurology, Yale University School of Medicine, New Haven, CT, USA ${ }^{e}$ Center for Neuroscience and Regeneration Research, Veterans Affairs Medical Center, West Haven, CT, USA

Clinical Genetics. 2012;82(4):351-8. Published with permission from John Wiley and Sons 


\begin{abstract}
Small fiber neuropathy (SFN) is a disorder typically dominated by neuropathic pain and autonomic dysfunction, in which the thinly myelinated $A \delta$-fibers and unmyelinated C-fibers are selectively injured. The diagnosis SFN is based on a reduced intraepidermal nerve fiber density and/or abnormal thermal thresholds in quantitative sensory testing. The etiologies of SFN are diverse, although no apparent cause is frequently seen.

Recently, SCNgA-gene variants (single amino acid substitutions) have been found in $\sim 30 \%$ of a cohort of idiopathic SFN patients, producing gain-offunction changes in sodium channel $\mathrm{Na}_{\mathrm{v}} \mathrm{1} \cdot 7$, which is preferentially expressed in small diameter peripheral axons. Functional testing showed that these variants altered fast inactivation, slow inactivation or resurgent current and rendered dorsal root ganglion neurons hyperexcitable. In this review, we discuss the role of $\mathrm{Na}_{\mathrm{v}} \mathrm{T} .7$ in pain and highlight the molecular genetics and pathophysiology of SCN9A-gene variants in SFN. With increasing knowledge regarding the underlying pathophysiology in SFN, the development of specific treatment in these patients seems a logical target for future studies.
\end{abstract}


Small fiber neuropathy (SFN) is a disorder of the thinly myelinated $A \delta$-fibers and unmyelinated C-fibers and is typically dominated by neuropathic pain and autonomic dysfunction. ${ }^{1-6}$ Clinical signs of small-fiber damage include loss of pinprick sensation, thermal sensory loss, allodynia or hyperalgesia. In pure SFN, large diameter fibers are spared, reflected by preservation of muscle strength, tendon reflexes, light touch, proprioceptive and vibratory sense in combination with normal nerve conduction studies.-7 In addition to the clinical picture, the diagnosis of SFN can be confirmed by demonstration of a reduced intraepidermal nerve fiber density in skin biopsy and/or abnormal thermal thresholds in quantitative sensory testing. ${ }^{7} 8$ After the diagnosis is made, an underlying cause for SFN has to be searched for, as some of these are potentially treatable. Potential causes for SFN are, among others, diabetes mellitus, impaired glucose tolerance, drugs and other toxins, Fabry disease, celiac disease, sarcoidosis, human immunodeficiency virus (HIV), and other systemic illnesses $4,6,7$

There is growing evidence for a genetic origin in various pain syndromes: an autosomal dominant inheritance has been shown in family members with burning feet syndrome, suggesting a genetic base for the small nerve fiber involvement in this disorder, although the locus responsible for the neuropathy has not been elucidated yet.9, 10 Gain-of-function mutations in the SCN9Agene, encoding the $\mathrm{Na}_{\mathrm{v}} \mathrm{\gamma} .7$ sodium channel, have been found in inherited erythromelalgia (IEM), ${ }^{11},{ }^{12}$ paroxysmal extreme pain disorder (PEPD), ${ }^{13}$ and recently in a substantial proportion of patients diagnosed with idiopathic SFN. ${ }^{14}$ These findings imply a pivotal role of $\mathrm{Na}_{\mathrm{v}} 1.7$ mutations in painful diseases and suggest an etiological basis for idiopathic SFN, whereby expression of gain-offunction sodium channel variants in small diameter peripheral axons produces pain and may cause these fibers to degenerate. In this review, the significance of $\mathrm{Na}_{\mathrm{V}}{ }^{1.7}$ in pain is discussed focusing on the role of $\mathrm{SCNgA-gene} \mathrm{variants} \mathrm{in}$ patients diagnosed with SFN, highlighting the molecular genetics and known pathophysiological evidence. In the near future, this new knowledge will result in the development of specific treatment for these patients.

\section{Voltage-gated sodium channels}

Just 60 years ago, Hodgkin and Huxley discovered the role of sodium channels in action potentials. ${ }^{15}$ In the decades that followed the primary structure and functional characteristics of these channels have been elucidated. ${ }^{16}$ These channels are large integral membrane polypeptides that are comprised of a large a subunit, which forms the voltage-sensitive and ion-selective pore, and smaller auxiliary $\beta$ subunit(s) that can regulate channel density in the cell 
membrane, and modulate the kinetics and voltage dependence of channel gating. ${ }^{16,17}$ The a subunit folds into four domains (I-IV), each of which contain six transmembrane segments, linked by three loops. In mammals, nine distinct voltage-gated sodium channels (VGSCs) a isoforms have been identified $\left(\mathrm{Na}_{\mathrm{v}}{ }^{1} \cdot 1-\mathrm{Na}_{\mathrm{v}}{ }^{1.9}\right)$, encoded by SCN1A-SCN $5 A$ and SCN8A-SCN11A genes. Besides their kinetics and voltage-dependent properties, they can be defined by their sensitivity to the sodium-channel blocker tetrodotoxin (TTX). VGSCs play a fundamental role in different types of excitable cells, including nerve, muscle and neuroendocrine cells. ${ }^{16,18}$

\section{Voltage-gated sodium channels and pain}

Dorsal root ganglia (DRG) and trigeminal neurons are the primary sensory neurons. Small DRG neurons give rise to the thinly myelinated $A \delta$-fibers and unmyelinated C-fibers which extend to the cutaneous layer. They provide the cold and warm sense, nociception and autonomic functions. ${ }^{19,20}$ In SFN these fibers are specifically involved.

More than one century ago, Charles Sherrington was the first who described the existence of nociceptors. ${ }^{21}$ Physiologically, in response to noxious stimuli the nociceptive neurons transmit pain signals to the central nervous system. Usually these cells are relatively quiescent and have high thresholds for activation. ${ }^{20}$ The excitability of the nociceptors seems to be controlled by different types of ion channels. ${ }^{22,}{ }^{23} \mathrm{Na}_{\mathrm{v}} 1.3, \mathrm{Na}_{\mathrm{v}} 1.7, \mathrm{Na}_{\mathrm{v}} 1.8$ and $\mathrm{Na}_{\mathrm{v}} 1.9$ are preferentially expressed in peripheral sensory neurons, whereby the last three are particularly located in nociceptive neurons. ${ }^{12,18,24-26}$ Dysregulated expression of the channels initiated by trauma, inflammation or metabolic disorders has been linked to chronic pain. ${ }^{27-33}$

$\mathrm{Na}_{\mathrm{V}} \mathrm{T} \cdot 3$ is only expressed in embryonic DRG, but is upregulated following axotomy in adults. ${ }^{34}$ Knockdown of $\mathrm{Na}_{\mathrm{v}} \mathrm{l} .3$ with antisense oligonucleotides and targeted shRNA has been reported to abrogate pain in rodent nerve injury models. ${ }^{35}, 36$ On the other hand, knockout of $\mathrm{Na}_{\mathrm{v}}{ }^{1} .3$ has been reported not to influence pain behavior after nerve injury. ${ }^{37}$ Despite clear upregulation of $\mathrm{Na}_{\mathrm{v}} \mathrm{l} \cdot 3$ expression in DRG, dorsal horn, and thalamic neurons after axonal injury, the role of $\mathrm{Na}_{\mathrm{v}} 1.3$ in pain remains controversial.

$\mathrm{Na}_{\mathrm{v}} 1.7, \mathrm{Na}_{\mathrm{v}} 1.8$, and $\mathrm{Na}_{\mathrm{v}} 1.9$ may show changes in the level of expression within injured human DRG neuron, although accumulation of $\mathrm{Na}_{\mathrm{v}} 1.7$ and $\mathrm{Na}_{\mathrm{v}} 1.8$ within injured axons in painful human neuromas was also described. ${ }^{88-43}$ The biophysical properties of $\mathrm{Na}_{\mathrm{v}} 1.8$, its critical role in repetitive firing, ${ }^{44}$ and its presence in free nerve endings, ${ }^{45}$ where painsignaling is initiated, suggest that $\mathrm{Na}_{\mathrm{v}} 1.8$ can significantly influence nociceptor excitability, thus contributing to pain. ${ }^{12}$ Experimental evidence supports a role for $\mathrm{Na}_{\mathrm{v}} 1.9$ in inflammatory and diabetic neuropathy pain, although a role in neuropathic pain is less clear. ${ }^{12}$ 
Expression of $\mathrm{Na}_{\mathrm{v}} 1.9$ has been shown to increase in DRG neurons innervating inflamed rat hindpaw..$^{46}$ Although expression levels of $\mathrm{Na}_{\mathrm{v}} \mathrm{T} .9$ do not appear to be altered in small DRG neurons from diabetic rats, increased $\mathrm{Na}_{\mathrm{v}} \mathrm{l} .9$ levels in large-diameter neurons suggest a contribution to painful diabetic neuropathy. ${ }^{47}$ In contrast, $\mathrm{Na}_{\mathrm{v}}$. 9 mRNA and protein levels and current density are downregulated in several animal models of neuropathic pain..$^{48-52}$ An early study using $\mathrm{Na}_{\mathrm{v}} 1.9$ antisense oligodeoxynucleotide (ODN) treatment did not report amelioration of neuropathic pain. ${ }^{53}$

\section{Voltage-gated sodium channel $\mathrm{Na}_{\mathrm{v}} \mathrm{1.7}$}

$\mathrm{Na}_{\mathrm{v}} \mathrm{T} .7$ was first isolated in 1995 as human neuroendocrine sodium channel (hNE-Na) from a human medullary thyroid carcinoma cell line. ${ }^{54}$ The suggestion that the channel is solely expressed in neuroendocrine cells was soon rejected by the isolation of the channel from rabbit Schwann cells (NaS). ${ }^{55}$ Subsequently, the rat homologue, also known as peripheral nerve type 1 or $\mathrm{PN} 1$, was cloned and showed to be preferentially expressed in DRG and sympathetic ganglion neurons. ${ }^{56,57} \mathrm{Na}_{\mathrm{v}} 1.7$ is encodedby the SCNgA-gene on chromosome $2 .{ }^{58}$ The human SCN9A-gene promoter has been identified and is located $\sim 64,000$ nucleotides upstream to the ATC translation start site. There is a high degree of sequence conservation between human and mouse. ${ }^{59} \mathrm{Na}_{\mathrm{v}} 1.7$ produces a fast activating and inactivating current that is TTX-sensitive (TTX-S $)^{54,56}$ and is slowrepriming from fast inactivation. ${ }^{60,}{ }^{61}$ Another electrophysiological feature of $\mathrm{Na}_{\mathrm{v}}{ }^{1.7}$ is the slow closed-state inactivation that generates a substantial current (ramp current) in response to small, slow depolarization. ${ }^{60,61}$ This ability to respond to ramp stimuli permits the channel to act as a threshold channel for firing action potentials, setting the gain in nociceptive neurons. ${ }^{62,63}$

\section{$\mathrm{Na}_{\mathrm{v}} 1.7$ and pain in animal studies}

Several animal models have been performed to study the contribution of $\mathrm{Na}_{\mathrm{v}} \mathrm{T} .7$ to acquired channelopathies. In rats, peripheral tissue inflammation induced by carrageenan or Freund's complete adjuvant, showed upregulation of $\mathrm{Na}_{\mathrm{v}} \mathrm{T} .7$ transcripts and protein, parallel with a significant increase in the level of sodium current in small DRG neurons. ${ }^{64-66}$ Inflammatory cytokines, such as nerve growth factor (NGF), also appear to upregulate $\mathrm{Na}_{\mathrm{v}} \mathrm{1} .7$ and might contribute to neuronal hyperexcitability.57, 67, 68 In accordance with these results, knockdown of $\mathrm{Na}_{\mathrm{v}} 1.7$ in primary afferents or DRG-neurons counteract the inflammationinduced mechanical and thermal hyperalgesia ${ }^{69,70}$ and plays a major role in pain after burn injury. ${ }^{71}$ In a recent study in experimental rat sciatic nerve neuromas, 2 weeks after nerve ligation and transaction, increased levels of $\mathrm{Na}_{\mathrm{v}} \mathrm{T} .7$ were shown and elevated levels of phosphorylated ERK $1 / 2$ within individual neuroma axons that exhibit $\mathrm{Na}_{\mathrm{v}} 1.7$ accumulation were shown. ${ }^{45}$ Neuropathic pain seems 
to persist after knockdown of $\mathrm{Na}_{\mathrm{v}} 1.7$ within DRG neurons, ${ }^{72}$ but is attenuated after knockdown of $\mathrm{Na}_{\mathrm{v}} 1.7$ in both DRG and sympathetic ganglion neurons. ${ }^{73}$ Alterations of sodium channel expression have also been linked to the development of painful diabetic neuropathy.47, 74-76 One study showed dysregulation of several sodium channels in streptozotocin (STZ)-induced diabetic neuropathy, although without change of $\mathrm{Na}_{\mathrm{v}} 1.7$ expression. ${ }^{47}$ In contrast, other studies just reported increased levels of $\mathrm{Na}_{\mathrm{v}} 1.7$ protein..$^{74,76}$ In addition, normalization of $\mathrm{Na}_{\mathrm{v}} \mathrm{\gamma} .7$ levels in STZ-diabetic rats, achieved by a non-replicating herpes simplex virus-based vector, substantially reduced painrelated behavior. ${ }^{77}$

\section{$\mathrm{Na}_{\mathrm{v}} 1.7$ and pain in human studies}

The contribution of $\mathrm{Na}_{\mathrm{v}} 1.7$ in traumatic injury has also been studied in human patients. Peripheral nerve injury showed reduction of $\mathrm{Na}_{v} 1.7$ in DRG neurons. ${ }^{39}$ Upregulation has been shown in painful neuromas. ${ }^{40,41,43}$

Much more is known about inherited pain disorders linked to mutations in the $\mathrm{SCN}_{9} A$-gene. Before the discovery of $S C \mathrm{NgA}_{9}$-gene variants in $\mathrm{SFN}, \mathrm{Na}_{\mathrm{v}} 1.7$ mutations have been identified in three pain syndromes (Fig. 1) of which the IEM is the most widely known. The clinical picture of IEM was first described in $1878^{78}$ and is characterized by a red discoloration of the extremities, aggravated by warmth and exercise. In 2004, missense mutations in the SCN9A-gene leading to gain-of-function of $\mathrm{Na}_{\mathrm{v}} \mathrm{T} .7$ were identified in two Chinese families. ${ }^{79}$ Since then multiple additional cases have been described. Most of the mutations show hyperpolarized activation, slow deactivation and increase the ramp response of the channel, ${ }^{80-84}$ which contributes to DRG neuron hyperexcitability. The second inherited human pain syndrome with gain-offunction mutations of $\mathrm{Na}_{\mathrm{v}} \mathrm{T} .7$ is PEPD. This autosomal dominant condition was first described in $1959 .{ }^{85}$ The clinical features consist of paroxysms of excruciating rectal, ocular and submaxillary pain associated with flushing of the buttocks, eyelid, periorbital skin and legs. ${ }^{13,85}$ Two years after the identification of the molecular basis of IEM, mutant $\mathrm{Na}_{\mathrm{v}} 1.7$ channels were found in PEPD.13 In addition to the difference in phenotype, the two conditions show differences in channel dysfunction, whereby mutant channels in PEPD show impaired fast inactivation, leading to prolonged action potentials and repetitive neuron firing in response to provoking stimuli, such as stretching and experiencing cold. ${ }^{13}$ In contrast with IEM and PEPD, the third syndrome, congenital insensitivity to pain (CIP) is an autosomal recessive condition characterized by loss-of-function $\mathrm{Na}_{\mathrm{v}} 1.7$ mutations leading to insensitivity to pain. ${ }^{86,87} \mathrm{CIP}$ is a disorder linked to different genetically targets. ${ }^{88}$ The patients who show a SCN9A-gene mutation form a subset that was first described in $2006,{ }^{86}$ and can be differentiated from other underlying causes by anosmia and absence of autonomic dysfunction. ${ }^{87}$ 
SFN shows some clinical similarities with IEM and PEPD. Burning pain is a common characteristic. Most SFN patients experience pain in the distal extremities such as IEM, ${ }^{14,79}$ but pain throughout the entire body or facial pain has also been described, the latter also seen in PEPD. 13, 14, 89 Despite the similarities, thus far SFN is considered a distinctive entity. For example, autonomic dysfunction is a prominent feature of SFN, whereas it is nearly absent in IEM.7, 11,90 In addition, skin reddening is typical for IEM, but is only occasionally present in SFN. ${ }^{14,91}$ The same applies for aggravation of symptoms by warmth and relief by cold in IEM. This is seen in some cases of SFN, ${ }^{14,91,92}$ but sometimes the opposite pattern is reported. ${ }^{14,89}$

Figure 1.

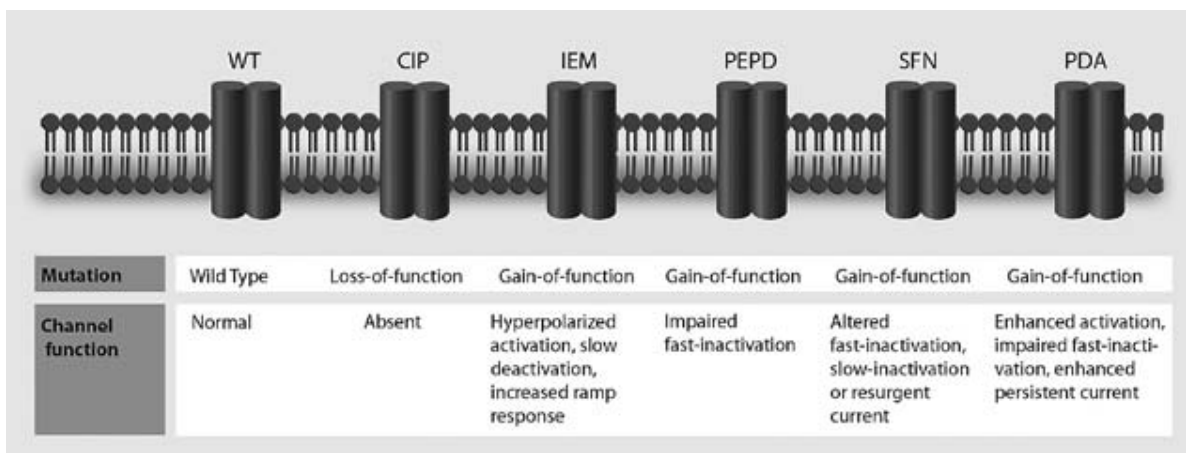

Legend to figure 1. $\mathrm{Na}_{\mathrm{v}} 1.7$ variants associated with clinical phenotype, mutation type and channel function. WT, wild type; CIP, congenital insensitivity to pain; IEM, inherited erythromelalgia; PEPD, paroxysmal extreme pain disorder; SFN, small fiber neuropathy; PDA, pain, dysautonomia and acromesomelia.

Given the shared characteristics of SFN with IEM and PEPD and the results of animal studies of VGSCs in pain as described above, the $S C N$ gA-gene was considered a potential candidate gene to be involved in idiopathic SFN. Recent research has confirmed this assumption. In a cohort of 28 Dutch Caucasian patients who met the strict criteria for idiopathic SFN based on reduced intraepidermal nerve fiber density plus abnormal quantitative sensory testing, eight showed gain-of-function SCNgA-gene variants. ${ }^{14}$ Subsequently, in a kindred with SFN and acromesomelia (small hands and feet) a gain-of-funtion SCNgA-gene variant was also shown. ${ }^{91}$

Increasing knowledge regarding pathophysiology may lead to novel therapeutic options. 


\section{Molecular genetics}

Up to now, in idiopathic SFN g SCN9A missense variants (single amino acid substitutions) have been reported ${ }^{14,89-92}$ (Table 1; Fig. 2). All variants substitute a highly conserved residue. Except for variant $\mathrm{M} 1532$ I which is located in domain IV of the channel, all other variants are situated in domain I or II. ${ }^{14,91}$ Two familial cases have been described, ${ }^{89,91}$ suggesting an autosomal dominant inheritance. However, there appears to be a variable penetrance as the complaints vary in severity between affected family members. Three variants have been reported in unrelated cases $\left(\mathrm{R} 185 \mathrm{H}, \mathrm{I} 739 \mathrm{~V}\right.$ and I228M). ${ }^{14}, 89$, 90 Variant I228M has been described in three patients, two being family members. ${ }^{14,89}$ While carrying the same mutation, the three patients present with different complaints, one with severe facial pain, the second with distal (feet, hands) pain, and the last with scalp discomfort. These patients show intra- and interfamily phenotypic diversity in patients with SFN produced by a gain-of-function variant of $\mathrm{Na}_{\mathrm{v}} 1.7$. One variant (G856D) not only resulted in SFN, but also caused a new syndrome of pain, dysautonomia and acromesomelia. ${ }^{91}$

Figure 2.

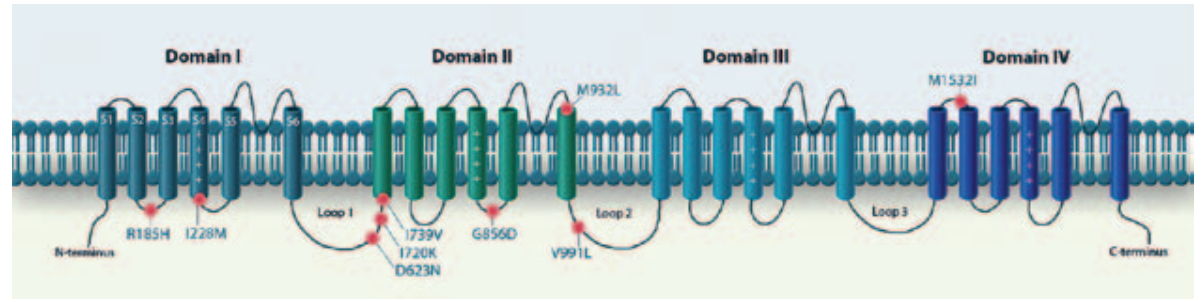

Legend to figure 2. Schematic sodium channel showing the locations of $\mathrm{Na}_{\mathrm{v}} 1.7$ variants found in patients with small fiber neuropathy.

\section{Molecular pathophysiology}

Voltage clamp and current clamp studies have been carried out to profile the effects of $\mathrm{Na}_{\mathrm{v}} 1.7$ variants in SFN on channel function and on DRG neuron firing properties. ${ }^{14,93,94}$ Because functional properties of sodium channel can best be studied within the cells where they are normally expressed in, voltage clamp analysis was carried out after transfection into adult small DRG neurons, ${ }^{14}$ for some of the variants, when biophysical changes were not detected in a HEK293 cell background. ${ }^{14,93,94}$ Current clamp analysis was carried out after transfection in DRG neurons. ${ }^{93,94}$ 
Table 1. SCN9A-gene variants in patients diagnosed with small fiber neuropathy

\begin{tabular}{|c|c|c|c|c|c|c|c|}
\hline \multirow[b]{2}{*}{$\begin{array}{l}\text { Nucleotide } \\
\text { change }\end{array}$} & \multirow[b]{2}{*}{ Protein } & \multirow[b]{2}{*}{ Domain } & \multirow[b]{2}{*}{$\begin{array}{l}\text { Number } \\
\text { of patients }\end{array}$} & \multirow[b]{2}{*}{ Reference(s) } & \multicolumn{3}{|c|}{ Allele frequency } \\
\hline & & & & & dbSNP & $\begin{array}{c}1000 \text { genomes } \\
\text { project }\end{array}$ & EVS \\
\hline c. $554 \mathrm{G}>\mathrm{A}$ & $\mathrm{R} 185 \mathrm{H}$ & I & 2 & $(14,90)$ & $\begin{array}{c}0.5 \% \\
(n=3 / 585)\end{array}$ & $\begin{array}{c}0.6 \% \\
(n=13 / 2184)\end{array}$ & $\begin{array}{c}0.6 \% \\
(n=68 / 11908)\end{array}$ \\
\hline$c .1867 \mathrm{G}>\mathrm{A}$ & $\mathrm{D} 623 \mathrm{~N}$ & II & 1 & (14) & - & - & - \\
\hline c. $2215 A>C$ & I739V & II & 3 & $(14,90,92)$ & $\begin{array}{c}0.2 \% \\
(n=1 / 571)\end{array}$ & $\begin{array}{c}0.09 \% \\
(n=2 / 2184)\end{array}$ & $\begin{array}{c}0.3 \% \\
(n=36 / 11838)\end{array}$ \\
\hline c. $2159 \mathrm{~T}>\mathrm{A}$ & I720K & II & 1 & (14) & - & $\begin{array}{c}0.01 \% \\
(n=1 / 9464)\end{array}$ & $\begin{array}{c}0.2 \% \\
(n=2 / 11762)\end{array}$ \\
\hline c. $4596 \mathrm{G}>\mathrm{A}$ & M1532I & IV & 1 & (14) & - & - & - \\
\hline c. $2794 A>C$ & M932L"\# & II & 1 & (14) & $\begin{array}{c}3.7 \% \\
(n=82 / 2188)\end{array}$ & $\begin{array}{c}3.7 \% \\
(n=81 / 2184)\end{array}$ & $\begin{array}{c}0.3 \% \\
(n=44 / 13006)\end{array}$ \\
\hline$c .2971 G>T$ & $V_{991} L^{\#}$ & II & 1 & (14) & $\begin{array}{c}3.7 \% \\
(n=82 / 2188)\end{array}$ & $\begin{array}{c}3.7 \% \\
(n=81 / 2184)\end{array}$ & $\begin{array}{c}0.4 \% \\
(n=42 / 11764)\end{array}$ \\
\hline c. $684 C>C$ & $1228 \mathrm{M}$ & I & $3^{\%}$ & $(14,89)$ & $\begin{array}{c}50 \% \\
(n=1 / 2) *\end{array}$ & $\begin{array}{c}0.05 \% \\
(n=1 / 2184)\end{array}$ & $\begin{array}{c}0.1 \% \\
(n=15 / 12988)\end{array}$ \\
\hline c. $2567 \mathrm{G}>\mathrm{A}$ & G856D & II & $3^{5}$ & (91) & - & - & - \\
\hline
\end{tabular}

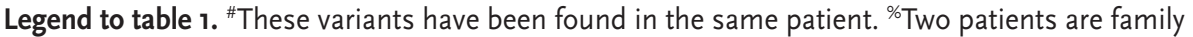
members. ${ }^{\$}$ All patients are of the same kindred. *This variant has only been found in one patient in this database.

Voltage clamp analysis of the first published variant channels in idiopathic SFN showed that they were all gain-of-function mutations and impaired slow inactivation (I720K, M1532I, I228M, I739V), depolarized slow and fast inactivation (D623N), or enhanced resurgent currents (M932L/V991L, $\mathrm{R} 185 \mathrm{H}) .{ }^{14}$ They did not display the hyperpolarized activation and enhanced ramp responses characteristic of IEM mutations or the incomplete fast inactivation characteristic of PEPD mutations." After current clamp analysis all of these variants produced hyperexcitability and abnormal spontaneous activity within DRG neurons. ${ }^{14}$

Several mutations show a distinct phenotype. Strikingly, the two patients housing variant $\mathrm{R} 185 \mathrm{H}$ showed minimal autonomic dysfunction compared to the other patients. ${ }^{14,90}$ Therefore, the biophysical effects of this variant were also assessed by voltage clamp in sympathetic ganglion neurons (superior cervical ganglia). A variant associated with severe autonomic symptoms (1739V) was 
used as comparator. ${ }^{14,90}$ As would be expected on the basis of the clinic, the $\mathrm{R} 185 \mathrm{H}$ variant did not produce detectable changes in properties of sympathetic ganglion neurons, in contrast with the $1739 \mathrm{~V}$ variant which had a profound effect on excitability of sympathetic ganglion neurons by increasing their current threshold and impairing their ability to generate repetitive activity, ${ }^{90}$ providing a biophysical basis for the clinical picture.

Current clamp analysis has also been carried out for some $\mathrm{Na}_{\mathrm{v}} 1.7$ variants in trigeminal ganglion neurons. One of the patients carrying variant $1228 \mathrm{M}$ complained of severe facial pain. ${ }^{14,89}$ As predicted, the $1228 \mathrm{M}$ variant produced hyperexcitability in trigeminal ganglion neurons..$^{89}$

Mechanisms by which some $\mathrm{Na}_{\mathrm{v}} 1.7$ variants produce, or increase risk for, axonal degeneration are under study, and may involve calcium loading, perhaps in response to a double hit by an additional stressor.

As noted above, variant G856D underlies an extraordinary phenotype consisting of SFN and acromesomelia. The $\mathrm{Na}_{\mathrm{v}} \mathrm{T} .7$ variant enhances activation, impairs fast inactivation and markedly enhances persistent current and the response to slow ramp stimuli.91 Limb underdevelopment has previously not been associated with $\mathrm{Na}_{\mathrm{v}}$ 1.7. Multiple hypotheses might be considered to explain the small limbs in this kindred. $\mathrm{Na}_{\mathrm{v}} 1.7$ channel might be involved in the bone ontogenesis, similar to $\mathrm{Na}_{\mathrm{v}} 1.2$ sodium channels which are expressed in osteoblasts during embryogenesis. ${ }^{95}$ Another hypothesis suggests that dysfunction or injury of peripheral nerve fibers due to DRG hyperexcitability may have interfered with normal limb development. Consistent with this suggesting, brachial plexus neurectomy in newborn rabbits has been shown to result in impaired limb growth. ${ }^{96}$ Altered vasomotor function, as shown by the skin reddening in the G586D kindred, may also play a role. The variant may have an effect on sympathetic ganglion neurons. ${ }^{77}$ In addition, bones are innervated by small nerve fibers, some of which contain peptides which have been implicated as modulators of the metabolism of bone cells. ${ }^{98,99}$ Moreover, nerve fibers are often located in the epiphysial region suggesting a regulatory role for the epiphysial growth plate. ${ }^{100}$ Finally, some conditions are known to cause both neuropathy and limb defects in embryo. One of the most wellknown examples is the limb teratogenesis produced by thalidomide. ${ }^{101}$ There is evidence suggesting that maternal varicella-zoster infection ${ }^{102}$ or maternal diabetic ${ }^{103}$ may also be teratogenic. Further work will be needed to delineate the role for the G856 $\mathrm{D} \mathrm{Na}_{\mathrm{v}} 1.7$ variant in this syndrome. 


\section{Conclusions}

SCN9A-gene variants (single amino acid substitutions) have recently been found in patients with SFN. These variants produce gain-of-function changes in sodium channel $\mathrm{Na}_{\mathrm{v}} \mathrm{T} .7$, which is preferentially expressed in small diameter peripheral axons. These variants alter fast inactivation, slow inactivation or resurgent current and render the dorsal root ganglion neurons hyperexcitable. Selective $\mathrm{Na}_{\mathrm{v}} \mathrm{T} .7$ blockers are currently under development. Given the profound changes seen in DRG neurons carrying these $\mathrm{Na}_{\mathrm{v}} 1.7$ variants, targeted block of $\mathrm{Na}_{\mathrm{v}} 1.7$ may prove to be effective in SFN, especially in patients with $S C \mathrm{NgA}_{9}$ mutations. 


\section{References}

1. Gorson, K.C. \& Ropper, A.H. Idiopathic distal small fiber neuropathy. Acta Neurol Scand 92, 376-82 (1995).

2. Holland, N.R. et al. Small-fiber sensory neuropathies: clinical course and neuropathology of idiopathic cases. Ann Neurol 44, 47-59 (1998).

3. Stewart, J.D., Low, P.A. \& Fealey, R.D. Distal small fiber neuropathy: results of tests of sweating and autonomic cardiovascular reflexes. Muscle Nerve 15, 661-5 (1992).

4. Lacomis, D. Small-fiber neuropathy. Muscle Nerve 26, 173-88 (2002).

5. Devigili, G. et al. The diagnostic criteria for small fibre neuropathy: from symptoms to neuropathology. Brain 131, 1912-25 (2008).

6. Lauria, G. Small fibre neuropathies. Curr Opin Neurol 18, 591-7 (2005).

7. Hoeijmakers, J.G., Faber, C.G., Lauria, G., Merkies, I.S. \& Waxman, S.G. Small-fibre neuropathies-advances in diagnosis, pathophysiology and management. Nat Rev Neurol 8, 369-79 (2012).

8. Tesfaye, S. et al. Diabetic neuropathies: update on definitions, diagnostic criteria, estimation of severity, and treatments. Diabetes Care 33, 2285-93 (2010).

9. Stogbauer, F. et al. Autosomal dominant burning feet syndrome. J Neurol Neurosurg Psychiatry 67, 78-81 (1999).

10. Kuhlenbaumer, G. et al. A second family with autosomal dominant burning feet syndrome. Ann N Y Acad Sci 883, 445-8 (1999).

11. Drenth, J.P. \& Waxman, S.G. Mutations in sodium-channel gene SCNgA cause a spectrum of human genetic pain disorders. J Clin Invest 117, 3603-9 (2007).

12. Dib-Hajj, S.D., Cummins, T.R., Black, J.A. \& Waxman, S.G. Sodium channels in normal and pathological pain. Annu Rev Neurosci 33, 325-47 (2010).

13. Fertleman, C.R. et al. SCNgA mutations in paroxysmal extreme pain disorder: allelic variants underlie distinct channel defects and phenotypes. Neuron 52, 767-74 (2006).

14. Faber, C.G. et al. Gain of function $\mathrm{Na}(\mathrm{V}) 1.7$ mutations in idiopathic small fiber neuropathy. Ann Neurol 7 , 26-39 (2012).

15. Hodgkin, A.L. \& Huxley, A.F. A quantitative description of membrane current and its application to conduction and excitation in nerve.J Physiol 117, 500-44 (1952).

16. Catterall, W.A. From ionic currents to molecular mechanisms: the structure and function of voltage-gated sodium channels. Neuron 26, 13-25 (2000).

17. Yu, F.H. \& Catterall, W.A. Overview of the voltage-gated sodium channel family. Genome Biol 4, 207 (2003).

18. Catterall, W.A., Goldin, A.L. \& Waxman, S.G. International Union of Pharmacology. XLVII. Nomenclature and structure-function relationships of voltage-gated sodium channels. Pharmacol Rev 57, 397-409 (2005).

19. McGlone, F. \& Reilly, D. The cutaneous sensory system. Neurosci Biobehav Rev 34, 148-59 (2010).

20. Lawson, S.N. Phenotype and function of somatic primary afferent nociceptive neurones with C-, Adeltaor Aalpha/beta-fibres. Exp Physiol 87, 239-44 (2002).

21. Sherrington, C.S. (ed.) The integrative action of the nervous sytem. (Yale University Press, New Haven, CT, 1906).

22. McCleskey, E.W. \& Gold, M.S. Ion channels of nociception. Annu Rev Physiol 61, 835-56 (1999).

23. Lee, Y., Lee, C.H. \& Oh, U. Painful channels in sensory neurons. Mol Cells 20, 315-24 (2005).

24. Djouhri, L. et al. The TTX-resistant sodium channel Nav1.8 (SNS/PN3): expression and correlation with membrane properties in rat nociceptive primary afferent neurons. J Physiol 550, 739-52 (2003).

25. Fang, $\mathrm{X}$. et al. The presence and role of the tetrodotoxin-resistant sodium channel $\mathrm{Na}(\mathrm{v}) 1.9(\mathrm{NaN})$ in nociceptive primary afferent neurons. J Neurosci 22, 7425-33 (2002).

26. Djouhri, L. et al. Sensory and electrophysiological properties of guinea-pig sensory neurones expressing Nav 1.7 (PN1) Na+ channel alpha subunit protein.J Physiol 546, 565-76 (2003).

27. Zhang, J.M., Donnelly, D.F., Song, X.J. \& Lamotte, R.H. Axotomy increases the excitability of dorsal root ganglion cells with unmyelinated axons.J Neurophysiol 78, 2790-4 (1997).

28. Wood, J.N., Boorman, J.P., Okuse, K. \& Baker, M.D. Voltage-gated sodium channels and pain pathways. J Neurobiol 61, 55-71 (2004). 
29. Waxman, S.C. Transcriptional channelopathies: an emerging class of disorders. Nat Rev Neurosci 2, 652-9 (2001).

30. Waxman, S.G. \& Hains, B.C. Fire and phantoms after spinal cord injury: $\mathrm{Na}+$ channels and central pain. Trends Neurosci 29, 207-15 (2006).

31. Lai, J., Porreca, F., Hunter, J.C. \& Gold, M.S. Voltage-gated sodium channels and hyperalgesia. Annu Rev Pharmacol Toxicol 44, 371-97 (2004).

32. Kajander, K.C., Wakisaka, S. \& Bennett, G.J. Spontaneous discharge originates in the dorsal root ganglion at the onset of a painful peripheral neuropathy in the rat. Neurosci Lett 138, 225-8 (1992).

33. Wall, P.D. \& Devor, M. Sensory afferent impulses originate from dorsal root ganglia as well as from the periphery in normal and nerve injured rats. Pain 17, 321-39 (1983).

34. Waxman, S.G., Kocsis, J.D. \& Black, J.A. Type III sodium channel mRNA is expressed in embryonic but not adult spinal sensory neurons, and is reexpressed following axotomy.J Neurophysiol 72, 466-70 (1994).

35. Hains, B.C., Saab, C.Y., Klein, J.P., Craner, M.J. \& Waxman, S.G. Altered sodium channel expression in second-order spinal sensory neurons contributes to pain after peripheral nerve injury. J Neurosci 24, 48329 (2004).

36. Samad, O.A. et al. Virus-mediated shRNA knockdown of $\mathrm{Na}(\mathrm{v}) 1.3$ in rat dorsal root ganglion attenuates nerve injury-induced neuropathic pain. Mol Ther 21, 49-56 (2013).

37. Nassar, M.A. et al. Nerve injury induces robust allodynia and ectopic discharges in Nav1.3 null mutant mice. Mol Pain 2, 33 (2006).

38. Coward, K. et al. Immunolocalization of $\mathrm{SNS}_{\mathrm{PN}} 3$ and $\mathrm{NaN} / \mathrm{SNS}_{2}$ sodium channels in human pain states. Pain 85, 41-50 (2000).

39. Coward, K. et al. Plasticity of TTX-sensitive sodium channels $\mathrm{PN}_{1}$ and brain III in injured human nerves. Neuroreport 12, 495-500 (2001).

40. Kretschmer, $\mathrm{T}$. et al. Accumulation of $\mathrm{PN}_{1}$ and $\mathrm{PN}_{3}$ sodium channels in painful human neuroma-evidence from immunocytochemistry. Acta Neurochir (Wien) 144, 803-10; discussion 810 (2002).

41. Bird, E.V., Robinson, P.P. \& Boissonade, F.M. Na(v)1.7 sodium channel expression in human lingual nerve neuromas. Arch Oral Biol 52, 494-502 (2007).

42. Black, J.A. et al. Upregulation of a silent sodium channel after peripheral, but not central, nerve injury in DRG neurons. J Neurophysiol 82, 2776-85 (1999).

43. Black, J.A., Nikolajsen, L., Kroner, K., Jensen, T.S. \& Waxman, S.G. Multiple sodium channel isoforms and mitogen-activated protein kinases are present in painful human neuromas. Ann Neurol 64, 644-53 (2008)

44. Renganathan, M., Cummins, T.R. \& Waxman, S.G. Contribution of $\mathrm{Na}(\mathrm{v}) 1.8$ sodium channels to action potential electrogenesis in DRG neurons. J Neurophysiol 86, 629-40 (2001).

45. Persson, A.K., Gasser, A., Black, J.A. \& Waxman, S.G. Nav1.7 accumulates and co-localizes with phosphorylated $\mathrm{ERK}_{1} / 2$ within transected axons in early experimental neuromas. Exp Neurol 230, 273-9 (2011).

46. Tate, S. et al. Two sodium channels contribute to the TTX-R sodium current in primary sensory neurons. Nat Neurosci 1, 653-5 (1998).

47. Craner, M.J., Klein, J.P., Renganathan, M., Black, J.A. \& Waxman, S.G. Changes of sodium channel expression in experimental painful diabetic neuropathy. Ann Neurol 52, 786-92 (2002).

48. Cummins, T.R. \& Waxman, S.G. Downregulation of tetrodotoxin-resistant sodium currents and upregulation of a rapidly repriming tetrodotoxin-sensitive sodium current in small spinal sensory neurons after nerve injury. J Neurosci 17, 3503-14 (1997).

49. Dib-Hajj, S.D., Tyrrell, L., Black, J.A. \& Waxman, S.G. NaN, a novel voltage-gated Na channel, is expressed preferentially in peripheral sensory neurons and down-regulated after axotomy. Proc Natl Acad Sci U S A 95, 8963-8 (1998).

50. Cummins, T.R., Black, J.A., Dib-Hajj, S.D. \& Waxman, S.G. Glial-derived neurotrophic factor upregulates expression of functional SNS and $\mathrm{NaN}$ sodium channels and their currents in axotomized dorsal root ganglion neurons. J Neurosci 20, 8754-61 (2000).

51. Decosterd, I., Ji, R.R., Abdi, S., Tate, S. \& Woolf, C.). The pattern of expression of the voltage-gated sodium channels $\mathrm{Na}(\mathrm{v}) 1.8$ and $\mathrm{Na}(\mathrm{v}) 1.9$ does not change in uninjured primary sensory neurons in experimental neuropathic pain models. Pain 96, 269-77 (2002).

52. Sleeper, A.A. et al. Changes in expression of two tetrodotoxin-resistant sodium channels and their currents in dorsal root ganglion neurons after sciatic nerve injury but not rhizotomy.J Neurosci 20, 7279 89 (2000). 
53. Porreca, F. et al. A comparison of the potential role of the tetrodotoxin-insensitive sodium channels, $\mathrm{PN}_{3} / \mathrm{SNS}$ and NaN/SNS2, in rat models of chronic pain. Proc Natl Acad Sci U S A 96, $7640-4$ (1999).

54. Klugbauer, N., Lacinova, L., Flockerzi, V. \& Hofmann, F. Structure and functional expression of a new member of the tetrodotoxin-sensitive voltage-activated sodium channel family from human neuroendocrine cells. EMBOJ 14, 1084-90 (1995).

55. Belcher, S.M., Zerillo, C.A., Levenson, R., Ritchie, J.M. \& Howe, J.R. Cloning of a sodium channel alpha subunit from rabbit Schwann cells. Proc Natl Acad Sci U S A 92, 11034-8 (1995).

56. Sangameswaran, L. et al. A novel tetrodotoxin-sensitive, voltage-gated sodium channel expressed in rat and human dorsal root ganglia.J Biol Chem 272, $14805-9$ (1997).

57. Toledo-Aral, J.). et al. Identification of $\mathrm{PN}_{1}$, a predominant voltage-dependent sodium channel expressed principally in peripheral neurons. Proc Natl Acad Sci U S A 94, 1527-32 (1997).

58. Beckers, M.C. et al. A new sodium channel alpha-subunit gene (Scnga) from Schwann cells maps to the Scn1a, Scn2a, Scn3a cluster of mouse chromosome 2. Genomics 36, 202-5 (1996).

59. Diss, J.K., Calissano, M., Gascoyne, D., Djamgoz, M.B. \& Latchman, D.S. Identification and characterization of the promoter region of the Nav1.7 voltage-gated sodium channel gene (SCNgA). Mol Cell Neurosci 37, 537-47 (2008).

6o. Cummins, T.R., Howe, J.R. \& Waxman, S.G. Slow closed-state inactivation: a novel mechanism underlying ramp currents in cells expressing the hNE/PN1 sodium channel. J Neurosci 18, 9607-19 (1998).

61. Herzog, R.I., Cummins, T.R., Ghassemi, F., Dib-Hajj, S.D. \& Waxman, S.G. Distinct repriming and closedstate inactivation kinetics of Nav1.6 and Nav1.7 sodium channels in mouse spinal sensory neurons. $J$ Physiol 551, 741-50 (2003).

62. Rush, A.M., Cummins, T.R. \& Waxman, S.G. Multiple sodium channels and their roles in electrogenesis within dorsal root ganglion neurons. J Physiol 579, 1-14 (2007).

63. Waxman, S.G. Neurobiology: a channel sets the gain on pain. Nature 444, 831-2 (2006).

64. Strickland, I.T. et al. Changes in the expression of $\mathrm{NaV}_{1.7}, \mathrm{NaV}_{1} .8$ and $\mathrm{NaV}_{1} .9$ in a distinct population of dorsal root ganglia innervating the rat knee joint in a model of chronic inflammatory joint pain. Eur J Pain 12, 564-72 (2008).

65. Black, J.A., Liu, S., Tanaka, M., Cummins, T.R. \& Waxman, S.G. Changes in the expression of tetrodotoxinsensitive sodium channels within dorsal root ganglia neurons in inflammatory pain. Pain 108, 237-47 (2004).

66. Gould, H.J., 3rd et al. Ibuprofen blocks changes in $\mathrm{Na} v 1.7$ and 1.8 sodium channels associated with complete Freund's adjuvant-induced inflammation in rat. J Pain 5, 270-80 (2004).

67. Toledo-Aral, J.J., Brehm, P., Halegoua, S. \& Mandel, G. A single pulse of nerve growth factor triggers longterm neuronal excitability through sodium channel gene induction. Neuron 14, 607-11 (1995).

68. Gould, H.J., 3rd et al. A possible role for nerve growth factor in the augmentation of sodium channels in models of chronic pain. Brain Res 854, 19-29 (2000)

69. Yeomans, D.C. et al. Decrease in inflammatory hyperalgesia by herpes vector-mediated knockdown of Nav1.7 sodium channels in primary afferents. Hum Gene Ther 16, 271-7 (2005).

70. Nassar, M.A. et al. Nociceptor-specific gene deletion reveals a major role for $\mathrm{Nav1}_{\text {.7 }}(\mathrm{PN} 1)$ in acute and inflammatory pain. Proc Natl Acad Sci U S A 101, 12706-11 (2004).

71. Shields, S.D. et al. Sodium channel $\mathrm{Na}(\mathrm{v}) 1.7$ is essential for lowering heat pain threshold after burn injury. J Neurosci 32, 10819-32 (2012).

72. Nassar, M.A., Levato, A., Stirling, L.C. \& Wood, J.N. Neuropathic pain develops normally in mice lacking both $\mathrm{Na}(\mathrm{v}) 1.7$ and $\mathrm{Na}(\mathrm{v})$ 1.8. Mol Pain 1, 24 (2005).

73. Minett, M.S. et al. Distinct Nav1.7-dependent pain sensations require different sets of sensory and sympathetic neurons. Nat Commun 3, 791 (2012).

74. Sun, W. et al. Reduced conduction failure of the main axon of polymodal nociceptive C-fibres contributes to painful diabetic neuropathy in rats. Brain 135, 359-75 (2012).

75. Okuse, K. et al. Regulation of expression of the sensory neuron-specific sodium channel SNS in inflammatory and neuropathic pain. Mol Cell Neurosci 10, 196-207 (1997).

76. Hong, S., Morrow, T.J., Paulson, P.E., Isom, L.L. \& Wiley, J.W. Early painful diabetic neuropathy is associated with differential changes in tetrodotoxin-sensitive and -resistant sodium channels in dorsal root ganglion neurons in the rat. J Biol Chem 279, 29341-50 (2004).

77. Chattopadhyay, M., Zhou, Z., Hao, S., Mata, M. \& Fink, D.J. Reduction of voltage gated sodium channel protein in DRG by vector mediated miRNA reduces pain in rats with painful diabetic neuropathy. $\mathrm{Mol}$ Pain 8, 17 (2012). 
78. Mitchell, S.W. On a rare vaso-motor neurosis of the extremities, and on the maladies with which it may be confounded. Am J Med Sci 17-36 (1878).

79. Yang, Y. et al. Mutations in $\mathrm{SCN} 9 \mathrm{~A}$, encoding a sodium channel alpha subunit, in patients with primary erythermalgia.J Med Genet 41, 171-4 (2004).

80. Cummins, T.R., Dib-Hajj, S.D. \& Waxman, S.G. Electrophysiological properties of mutant Nav1.7 sodium channels in a painful inherited neuropathy. J Neurosci 24, 8232-6 (2004).

81. Dib-Hajj, S.D. et al. Gain-of-function mutation in Nav1.7 in familial erythromelalgia induces bursting of sensory neurons. Brain 128, 1847-54 (2005).

82. Han, C. et al. Sporadic onset of erythermalgia: a gain-of-function mutation in Nav1.7. Ann Neuro/ 59, 553-8 (2006).

83. Harty, T.P. et al. $\mathrm{Na}(\mathrm{V}) 1.7$ mutant $\mathrm{A} 86{ }_{3} \mathrm{P}$ in erythromelalgia: effects of altered activation and steady-state inactivation on excitability of nociceptive dorsal root ganglion neurons. J Neurosci 26, 12566-75 (2006).

84. Lampert, A., Dib-Hajj, S.D., Tyrrell, L. \& Waxman, S.G. Size matters: Erythromelalgia mutation S241T in Nav1.7 alters channel gating. J Biol Chem 281, 36029-35 (2006).

85. Hayden, R. \& Grossman, M. Rectal, ocular, and submaxillary pain; a familial autonomic disorder related to proctalgia fugaz: report of a family. AMAJ Dis Child 97, 479-82 (1959).

86. Cox, J.J. et al. An SCNgA channelopathy causes congenital inability to experience pain. Nature $444,894-8$ (2006).

87. Goldberg, Y.P. et al. Loss-of-function mutations in the Nav1.7 gene underlie congenital indifference to pain in multiple human populations. Clin Genet 71, 311-9 (2007).

88. Rotthier, A. et al. Genes for hereditary sensory and autonomic neuropathies: a genotype-phenotype correlation. Brain 132, 2699-711 (2009).

89. Estacion, M. et al. Intra- and interfamily phenotypic diversity in pain syndromes associated with a gainof-function variant of $\mathrm{NaV}$ 1.7. Mol Pain 7, 92 (2011).

90. Han, C. et al. Functional profiles of $\mathrm{SCN}_{9} \mathrm{~A}$ variants in dorsal root ganglion neurons and superior cervical ganglion neurons correlate with autonomic symptoms in small fibre neuropathy. Brain 135, 2613-28 (2012).

91. Hoeijmakers, J.G. et al. Small nerve fibres, small hands and small feet: a new syndrome of pain, dysautonomia and acromesomelia in a kindred with a novel $\mathrm{NaV}$.7 mutation. Brain 135, 345-58 (2012).

92. Han, C. et al. Nav1.7-related small fiber neuropathy: impaired slow-inactivation and DRG neuron hyperexcitability. Neurology 78, 1635-43 (2012).

93. Dib-Hajj, S.D. et al. Transfection of rat or mouse neurons by biolistics or electroporation. Nat Protoc 4, 1118-26 (2009).

94. Cummins, T.R., Rush, A.M., Estacion, M., Dib-Hajj, S.D. \& Waxman, S.G. Voltage-clamp and currentclamp recordings from mammalian DRG neurons. Nat Protoc 4, 1103-12 (2009).

95. Black, J.A., Westenbroek, R.E., Catterall, W.A. \& Waxman, S.G. Type II brain sodium channel expression in non-neuronal cells: embryonic rat osteoblasts. Brain Res Mol Brain Res 34, 89-98 (1995).

96. Alharby, S.W. Anterior cruciate ligament injuries in growing skeleton. Int J Health Sci (Qassim) 4, 71-9 (2010).

97. Rush, A.M. et al. A single sodium channel mutation produces hyper- or hypoexcitability in different types of neurons. Proc Natl Acad Sci U S A 103, 8245-50 (2006).

98. Jimenez-Andrade, J.M. et al. A phenotypically restricted set of primary afferent nerve fibers innervate the bone versus skin: therapeutic opportunity for treating skeletal pain. Bone 46, 306-13 (2010).

99. Castaneda-Corral, G. et al. The majority of myelinated and unmyelinated sensory nerve fibers that innervate bone express the tropomyosin receptor kinase A. Neuroscience 178, 196-207 (2011).

100. Hill, E.L. \& Elde, R. Distribution of CGRP-, VIP-, D beta H-, SP-, and NPY-immunoreactive nerves in the periosteum of the rat. Cell Tissue Res 264, 469-80 (1991).

101. Vargesson, N. Thalidomide-induced limb defects: resolving a 50-year-old puzzle. Bioessays 31, 1327-36 (2009).

102. Savage, M.O., Moosa, A. \& Gordon, R.R. Maternal varicella infection as a cause of fetal malformations. Lancet 1, 352-4 (1973).

103. Botek, G., Anderson, M.A. \& Taylor, R. Charcot neuroarthropathy: An often overlooked complication of diabetes. Cleve Clin J Med 77, 593-9 (2010). 

Chapter 9

\section{Summary, general discussion and future perspectives}


Small fiber neuropathy (SFN) is a challenging condition in terms of diagnosis, underlying pathophysiology and treatment.' In the last two decades, considerable progress has been made to establish the diagnosis SFN more reliably, which is of eminent importance for both clinical practice and research. Although several pathophysiological mechanisms have been proposed to cause SFN, the exact pathogenesis remains unclear, especially in the group of patients diagnosed with idiopathic SFN (I-SFN). As a result, appropriate treatment is generally lacking.

In this thesis, the possible role of voltage gated sodium channel (VCSC) mutations in the pathogenesis of SFN is described, which may serve as a starting point in the development of targeted therapeutic anti-neuropathic agents.

\section{Summary and general discussion}

Chapter 1 is a general introduction describing SFN and the role and function of sodium channels. The aims and outline of the thesis are provided.

Chapter $\mathbf{2}$ is a review on SFN, focusing on diagnosis, pathophysiology and management. SFN is a disorder of thinly myelinated $A \delta$-fibers and unmyelinated C-fibers, and is clinically characterized by neuropathic pain symptoms and autonomic complaints.' Diagnosis of SFN is challenging as the clinical picture can be difficult to interpret and results from nerve conduction studies are often normal. In cases of suspected SFN, measurement of intraepidermal nerve fiber density (IENFD) and/or analysis of quantitative sensory testing (QST) can enable diagnosis. New diagnostic techniques (including measurement of nerve fiber density using corneal confocal microscopy, and nociceptive evoked potentials) may contribute to the diagnostic work-up. SFN can be associated with systemic diseases such as immune-mediated disorders, but remains idiopathic in a substantial proportion of patients. Improved understanding of the pathogenesis of the disorder will contribute to better patient management.

In chapter 3 missense mutations in the $\mathrm{SCN}_{9 A}$-gene, which encodes $\mathrm{Na}_{\mathrm{v}}{ }^{1.7}$, are described in 8 of $28(28.6 \%)$ patients with skin biopsy- and QST- confirmed I-SFN. ${ }^{2}$ Functional analyses showed gain-of-function changes of the mutant channels, resulting in impaired slow inactivation, depolarized slow and fast inactivation or enhanced resurgent currents. All mutations rendered the dorsal root ganglion (DRG) neurons hyperexcitable.

The $\mathrm{Na}_{\mathrm{v}} \mathrm{\gamma} .7$ mutations and their specific characteristics, can explain the clinical picture of a substantial part of patients diagnosed with I-SFN. Although these findings will not alter the treatment strategy immediately, it has been shown that 
just certainty about the origin of symptoms is of great significance for patients as well ("Knowing for the sake of knowing"). ${ }^{3}$ Advice about lifestyle modifications can be as much a treatment as medication or surgical interventions. ${ }^{3}$ Moreover, in chronic pain studies it has been demonstrated that when symptom certainty is high, supported by a medical cause for the pain, the doctor-relationship is better and clinical judgments and medical decisions are more straightforward. 4,5 In our study cohort, the mean duration of symptoms before presentation was 14.5 years. $^{2}$ A considerable number of the patients we studied had already been referred to the psychiatrist because of unexplained pain symptoms, unaltered physical examination and standard additional investigations, which had been interpreted as suggesting a psychosomatic disorder. Besides these findings, patients were labeled and stigmatized with an inaccurate psychiatric or behavioral diagnosis.

The finding of $\mathrm{Na}_{\mathrm{v}} 1.7$ mutations in SFN is also important for family members of affected patients, since they are at risk for harboring the mutated gene. Adequate genetic counseling is therefore mandatory. Family research in two of our studies suggested an autosomal dominant inheritance with a variable penetrance. 6,7 Currently, the clinical value of presymptomatic DNA-testing is questionable, because relatively little is known about the long-term course of the disease. Furthermore, therapy that can influence the progression of the condition is not yet available. However, providing the opportunity to examine family members with possible complaints of SFN is the focus of near future aims. Hopefully these future studies will determine whether presymptomatic testing is clinically useful.

In chapter 4 to 6 neuron type-specific effects of mutant channels have been demonstrated.

The $1228 \mathrm{M}$ mutation showed intra- and interfamilial variability (chapter 4). ${ }^{6}$ Current clamp analyses demonstrated hyperexcitability in trigeminal ganglion neurons, thus following the clinical location and complaints of the patients with severe facial pain. ${ }^{6}$

Chapter 5 describes the functional characteristics of mutation $1739 \mathrm{~V},{ }^{8}$ whereas differences in two mutations between voltage clamp and current clamp recordings in transfected superior cervical ganglion (SCC) neurons are described in chapter $6 .{ }^{9}$ Mutation $\mathrm{R} 185 \mathrm{H}$, found in patients with hardly any autonomic complaints, did not alter excitability of SGC neurons, whereas mutation $1739 \mathrm{~V}$, identified in patients with severe autonomic dysfunction, rendered SGC neurons hypoexcitable (therefore resulting in loss-of-function in the autonomic nervous system), thus again validating the clinical findings seen in these two mutations. ${ }^{9}$

The cell electrophysiological studies of the $\mathrm{Na}_{\mathrm{v}} \mathrm{T} .7$ mutations in I-SFN showed 
distinct profiles of DRG hyperexcitability and firing compared with the mutations described in inherited erythromelalgia (IEM) and paroxysmal extreme pain disorder (PEPD). ${ }^{2,8,10}$ Besides, for some mutations neuron type-specific effects have been demonstrated in trigeminal ganglion neurons and SCC neurons. ${ }^{6}$, ${ }_{9}$ Both the variety of biophysiological changes in DRG, and the differences in expression in specific types of neurons may cause variances in genotypes resulting into different phenotypes. However, intra- and interfamilial variability within one mutation, as shown for example in mutation I228M, cannot be explained solely by this phenomenon. ${ }^{6,7}$

Differences in clinical pattern between patients with a similar SCNgA mutation have been described before. In a kindred with IEM based on mutation G616R, the age of onset of symptoms differed between family members." Functional analyses indicated that developmental switching of splice isoforms of the sodium channel contributed to delayed onset of disease with symptoms appearing only after expression of a sufficient proportion of channels in the permissive splice variant. In addition, SCN9A mutation $\mathrm{W}_{1538 \mathrm{R}}$ has been described in two studies, in which one patient showed a late-onset chronic nonparoxysmal neuropathic pain phenotype and another patient the clinical picture of erythermalgia. ${ }^{2,13}$

Although SFN, IEM and PEPD have clear discriminating clinical features, they also show similarities (Table 1). In some patients diagnosed with a SCN9A mutation, the distinction between the three pain disorders may be difficult.

Figure 1. Spectrum of $\mathrm{SCN} g \mathrm{~A}$ related pain disorders

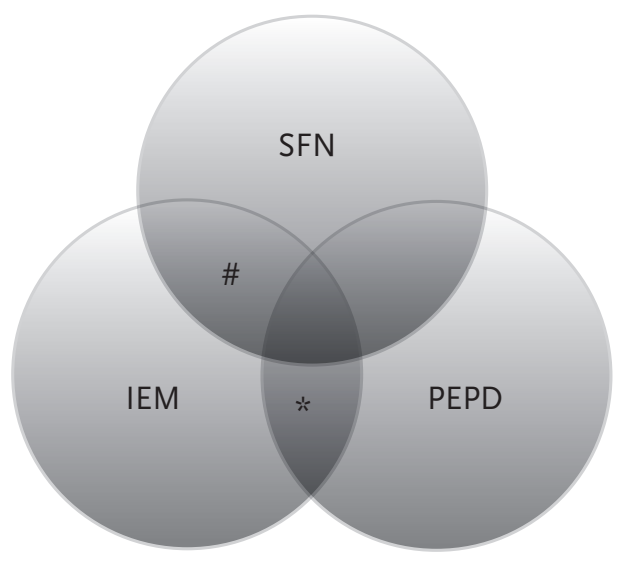

Legend to figure 1. SFN = small fiber neuropathy, IEM = inherited erythromelalgia, PEPD = paroxysmal extreme pain disorder. Two examples of $S C N g A$ mutations with overlapping syndromes: \# = SCNgA mutation $1228 \mathrm{M}$ has been described to cause both SFN as IEM symptoms ${ }^{6}, *=S C N 9 A$ mutation $\mathrm{A}_{1} 632 \mathrm{E}$ has been demonstrated to result in a mixed IEM and PEPD phenotype. ${ }^{14}$ 
For example, mutation $\mathrm{A}_{1} 632 \mathrm{E}$ is associated with a mixed clinical phenotype that includes features of both IEM and PEPD. Interestingly, also the electrophysiological properties of this mutation are characteristic for both

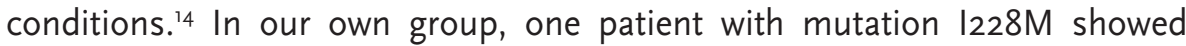
autonomic complaints compatible with SFN, but also burning red discolored extremities triggered by rising temperature and exercise, characteristic for IEM. ${ }^{6}$ It seems that the three $\mathrm{SCN}_{9}$ A gain-of-function-related pain disorders could be considered as part of a clinical and biophysical spectrum (Figure 1).

Table 1. Characteristics $S C N g A$ gain-of-function related pain disorders

\begin{tabular}{|c|c|c|c|}
\hline & SFN & IEM & PEPD \\
\hline Age of onset symptoms & $\begin{array}{c}\text { at any age, sometimes } \\
\text { even }>50 \text { years }\end{array}$ & $\begin{array}{l}\text { usually }<6 \text { years, } \\
\text { late onset } 2^{\text {nd }} \text { decade }\end{array}$ & $\begin{array}{l}\text { at birth or } \\
\text { at childhood }\end{array}$ \\
\hline Burning pain & +++ & +++ & +++ \\
\hline $\begin{array}{l}\text { Pain location } \\
\text { Feet } \\
\text { Hands } \\
\text { Proximal extremities } \\
\text { Myalgia } \\
\text { Jaw/Facial pain } \\
\text { Ocular } \\
\text { Rectal }\end{array}$ & $\begin{array}{l}++( \pm) \\
++ \\
+( \pm) \\
+( \pm) \\
+( \pm) \\
- \\
-\end{array}$ & $\begin{array}{l}+++ \\
+( \pm) \\
- \\
- \\
- \\
- \\
-\end{array}$ & $\begin{array}{c}+ \\
- \\
+ \\
- \\
+++ \\
+++ \\
+++\end{array}$ \\
\hline Duration pattern & $\begin{array}{l}\text { continuous with } \\
\text { exacerbations }\end{array}$ & episodic in general & episodic \\
\hline Skin colorization & $+( \pm)$ & +++ & +++ \\
\hline Dry mouth and/or eyes & ++ & - & - \\
\hline Visual complaints & $+( \pm)$ & - & - \\
\hline Bowel complaints & ++ & - & - \\
\hline Palpitations & + & - & - \\
\hline Bradycardia/aystole & - & - & + \\
\hline Dizziness & + & $( \pm)$ & - \\
\hline Hot flashes & + & - & ++ \\
\hline Non epileptic seizures & - & - & ++ \\
\hline Warmth & $\downarrow$ pain or $\uparrow$ pain & $\uparrow$ pain & - \\
\hline Exercise & $\begin{array}{c}\text { no pain during exercise; } \\
+( \pm) \text {, pain may at rest } \\
\text { after exercise }\end{array}$ & $\uparrow$ pain & $\begin{array}{c}\uparrow \text { pain during } \\
\text { chewing }\end{array}$ \\
\hline Cold & $\downarrow$ pain or $\uparrow$ pain & $\downarrow$ pain & $\uparrow$ pain \\
\hline
\end{tabular}

Legenda to table 1. SFN = small fiber neuropathy, IEM = inherited erythromelalgia, PEPD $=$ paroxysmal extreme pain disorder,$+=$ present,$-=$ absent,$\pm=$ variable present or absent, $\downarrow=$ decrease, $\uparrow=$ increase 
Also, in other sodium channel dysfunction related diseases, such as $\mathrm{SCN}_{5} \mathrm{~A}$ associated cardiac disorders ${ }^{15}$ or $\mathrm{SCN} 1 \mathrm{~A}$-related epilepsy, ${ }_{1}^{16}$ phenotypic variability within one mutation has been demonstrated. This implicates that other factors may contribute to the differences seen in clinical picture between family members, such as particular modifier genes. Common $\mathrm{SCN}$ gA polymorphisms have been described to be associated with pain perception in a range of pathological conditions. ${ }^{17-19}$ Besides sodium channel genes, the gene encoding catechol-O-methyltransferase $(C O M T)^{20}$ and the CTP cyclohydrolase gene $\left(G C H_{1}\right)^{21}$ have been shown to influence pain sensitivity.

In SCN8A the disease modifier gene SCNM1 influences disease severity through an effect on splicing. ${ }^{22}$ Furthermore, molecules that modulate the physiological properties of sodium-channel isoforms, like fibroblast growth factor homologous factors do in SCN8A, may be of importance. ${ }^{23,}{ }^{24}$ Another point of view on phenotypic variability comes from the growing area of epigenetics. In chronic pain states environmental factors, such as pain experience, stress and memory of pain, could modulate genomic expression via DNA methylation, histone modifications, RNAi, and chromatin dynamics. ${ }^{25-27}$ This type of research is still in its infancy, but may be of great value in the future for a better understanding of the factors that play a role in the phenotypic characteristics of pain perception. Taken the above-mentioned information into account, possibly the existence of additional modifier genes, other molecules or epigenetic factors may play a role in the modulation of these variants into a pathogenetic state.

A striking phenotype has been shown in a kindred with the novel $\mathrm{Na}_{\mathrm{v}} 1.7$ mutation G856D in chapter 7, which enhances activation, impairs fast inactivation and markedly enhances persistent current and the response to slow ramp stimuli.? The combination of SFN, acromesomelia and autonomic dysfunction was only present in family members carrying this mutation. ${ }^{7}$ It is suggested that the mutation results in peripheral nerve dysfunction, which most probably contributes to the impaired limb development.

Chapter 8 is a review on the genetic aspects of SFN. The role of $\mathrm{Na}_{\mathrm{v}} 1.7$ in pain is discussed and the molecular genetics and pathophysiology of SCNgAgene variants in SFN is highlighted. With increasing knowledge regarding the underlying pathophysiology in SFN, the development of specific treatment in these patients seems a logical target for future studies. 


\section{Future perspectives}

In addition to the above, the section 'future perspectives' will mainly focus on two cardinal features, namely i: pathogenesis and related issues and ii: treatment options. These subdomains will be highlighting the need for future studies in SFN and in sodium channelopathies.

\section{Pathogenesis}

\section{Loss of intraepidermal nerve fibers}

The molecular substrates for axonal injury and loss of IENF in I-SFN are not known. The spontaneous firing and increased evoked firing frequency of transfected DRG neurons in $\mathrm{Na}_{\mathrm{v}} 1.7$ mutations, may intuitively explain the clinical picture of SFN with spontaneous and evoked pain. ${ }^{2}$ However, loss of IENF, which is an important feature in diagnosing SFN, is not always seen and may not be directly explained by spontaneous firing of the neuron.

\section{Intraepidermal nerve fiber loss related to SCN9A mutations}

Besides $\mathrm{Na}_{\mathrm{v}} 1.7$, also $\mathrm{Na}_{\mathrm{v}} 1.6, \mathrm{Na}_{\mathrm{v}} 1.8, \mathrm{Na}_{\mathrm{v}} 1.9$ and the sodium-calcium exchanger NCX are expressed in the intraepidermal nerve terminals. ${ }^{28}$ In anoxic and ischemic situations it has been shown that the intracellular sodium concentration increases and causes peripheral nerve axon injury via an excess of axoplasmic calcium induced by calcium-importing reverse sodium-calcium exchange. ${ }^{29-31}$ Since $\mathrm{Na}_{\mathrm{v}} 1.7$ mutations in SFN also increase the sodium influx, the same process may cause IENF damage. In addition to some of the studies reported in this thesis, possible nerve axon injury was recently studied in vitro for the mutations $1228 \mathrm{M}, \mathrm{M} 932 \mathrm{~L} / \mathrm{V} 991 \mathrm{~L}$ and $1720 \mathrm{~K} .{ }^{32}$ The DRG transfected with I228M (Figure 2), showed a $20 \%$ reduced neurite length compared to wild-type channels. The other mutations demonstrated a trend toward reduced neurite length. In addition, carbamazepine and a reverse $\mathrm{Na}^{+}-\mathrm{Ca}^{2+}$ exchange blocker ameliorated this reduction in neurite length. Future studies should aim to demonstrate this mechanism in vivo, via the assessment of IENFD changes under treatment..$^{32}$ Such a model could also serve to test potential drugs in particular mutations before the steps taken to clinical evaluation.

\section{Functional analysis of mutations}

All $\mathrm{Na}_{\mathrm{v}} 1.7$ mutations described in this thesis were functional analyzed with voltage clamp and current clamp studies to prove their pathogenicity, and explain their pathophysiologic properties. ${ }^{2,6-9}$ However, these techniques are very time consuming and expensive. Because it is expected that the number of possible pathogenic mutations will rapidly increase, a more easily applicable 
Figure 2. Reduced neurite lengths in transfected dorsal root ganglion with $1228 \mathrm{M}$

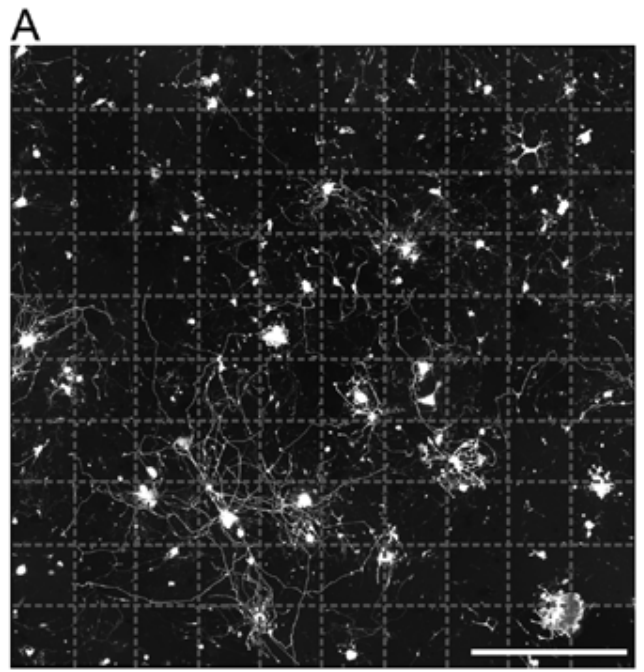

C

B
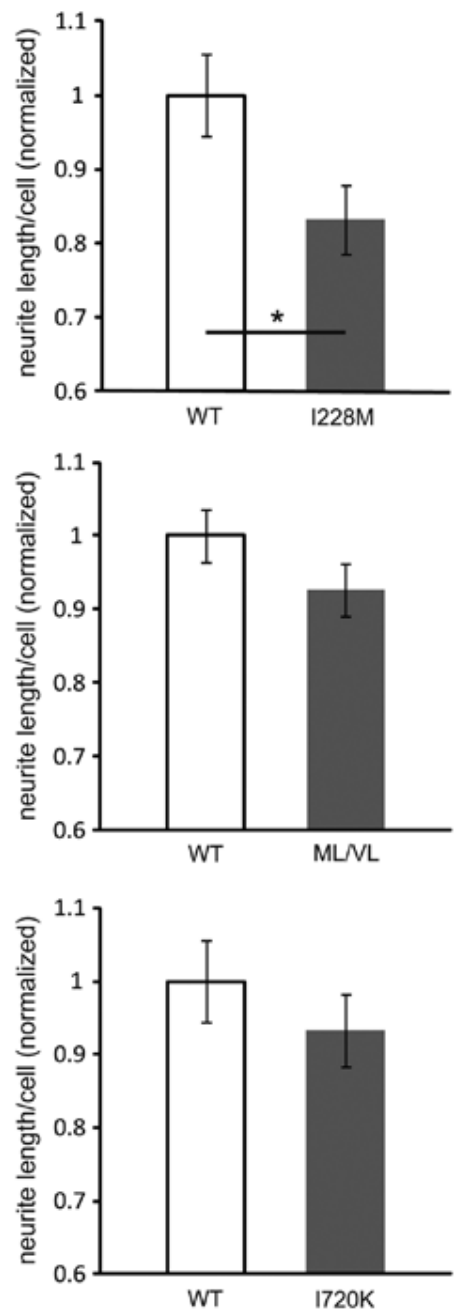

Legend to figure 2.Neurite length of neurons expressing $\mathrm{Na}_{\mathrm{v}} 1.7$ wild-type (WT) and I228M, M932L/V991 L (ML/VL), and I720K channels. (A) Large-field montage image consisting of a 10 310 field-of-view montage image of a dorsal root ganglion culture 3 days after transfection with $\mathrm{Na}_{\mathrm{v}} 1.7$ WT 1 green fluorescent protein (GFP) constructs, with GFP signal as white. Dotted lines distinguish individual field-of-view captures. Scale bar 5 1,00olM. (B) Increased magnification

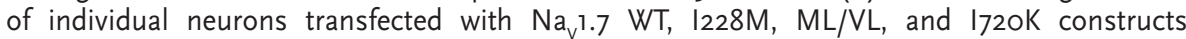
demonstrates reduced neurite length of $1228 \mathrm{M}$-transfected neuron compared to WT. Scale bar 5 250lM. (C) Quantifications of the total neurite length/neuron calculated from large-field images and averaged for each condition. Pairwise comparisons between neurites from neurons expressing WT channels and channel variants $1228 \mathrm{M}, \mathrm{ML} / \mathrm{VL}$, and $1720 \mathrm{~K}$ are presented. Data are normalized to WT values and presented as mean 6 standard error of the mean. $* p<0.05$. Published with permission from John Wiley and Sons. 
screening tool is needed. Zebrafish have been shown to be useful for modeling human diseases and for drug research, because of the high anatomic similarity with humans and rapid development of offsprings. ${ }^{33,34}$ Moreover, within the course of sodium channel studies, SCNA gene expression has been demonstrated. 35 Zebrafish larvae can detect a variety of thermal, chemical, and mechanical stimuli, including pain. Specially developed devices to assess movement and behavior reactions on these stimuli have already been used in other studies evaluating the role of ion channel mutations in nociception. ${ }^{36}$ In addition to the behavioral parameters, also morphological characteristics can be determined. In a study about the effects of vincristine and bortezomib in chemotherapy-induced polyneuropathy, reduction of the sensory axon density in the skin was observed. ${ }^{37}$ These features of the zebrafish model appear to be applicable for mutation analysis in SFN. Therefore, the zebrafish model may improve reliable screening of candidate pain-inducing gene variants as well as the possibility to examine the efficacy and toxicity of potential new drug substances.

\section{Other voltage gated sodium channels of interest}

SCNgA mutations were found in $28.6 \%$ of patients diagnosed with I-SFN. However, about $70 \%$ of the cases remain genetically unexplained. As already described in chapter $1, \mathrm{Na}_{\mathrm{v}} 1.8, \mathrm{Na}_{\mathrm{v}} 1.9, \mathrm{Na}_{\mathrm{v}} 1.6$ and $\mathrm{Na}_{\mathrm{v}} 1.3$ are also expressed in the small diameter DRG neurons. It is possible that mutations in these channels may play a role in a subset of the remaining $70 \%$. The identification of more genetic target sites and tailored pharmacologic molecules will contribute to better understanding of this illness and development of appropriate treatment.

$\mathrm{Na} a_{\mathrm{v}} 1.8$

$\mathrm{Na}_{\mathrm{v}} 1.8$ is encoded by the $S C \mathrm{~N}_{10} \mathrm{~A}$ gene..$^{38}$ The channel provides the major part of the current underlying the depolarization of the action potential in the cells in which the channel is expressed. ${ }^{39}, 40$ Besides DRG neurons, $\mathrm{Na}_{\mathrm{v}} 1.8$ is also present in trigeminal ganglia, ${ }^{41}$ peripheral axons shafts, ${ }^{42}$ free nerve terminals in the $\mathrm{skin}^{43}$ and cornea. ${ }^{44} \mathrm{Na}_{\mathrm{v}} 1.8$ and $\mathrm{Na}_{\mathrm{v}}$ 1.7 show interactions, in such way that changes in expression of both channels provide a regulatory mechanism that tunes the excitability of the DRG neurons. ${ }^{45}, 46$ Several studies have indicated the involvement of the $\mathrm{Na}_{\mathrm{v}} 1.8$ channel in the pathophysiology of pain. $\mathrm{Na}_{\mathrm{v}} 1.8$-knockout models proved that the channel is essential for mechanical, cold, and inflammatory pain sensation. ${ }^{47-49}$ In addition, antisense-mediated knock-down of $\mathrm{Na}_{\mathrm{v}} 1.8$ in rats, reversed neuropathic pain behavior after $L 5 / L 6$ spinal nerve ligation. ${ }^{50,51} \mathrm{An}$ upregulation of $\mathrm{Na}_{\mathrm{v}} 1.8$ has been shown in damaged rat sciatic nerve axons, ${ }^{52}$ but also in sciatic nerve axons after injury of L4 ganglia..$^{17}$ In humans, accumulation of the channel has been demonstrated in neuromas. ${ }^{53,54}$ In streptozocin-induced diabetic rats $\mathrm{Na}_{\mathrm{v}} 1.8$ plays a role in hyperalgesia, and systemic or local blockade of $\mathrm{Na}_{\mathrm{v}} 1.8$ channels was effective against hyperalgesia and allodynia. .556 
Based on these observations, following the studies in this thesis, our research group has performed SCN10A genomic analysis in 104 SCNgA negative painful predominantly SFN patients..$^{57}$ Seven novel $\mathrm{Na}_{\mathrm{v}} 7.8$ mutations were found in nine patients, which support further research in this field.

$N a_{v} 1 \cdot 9$

The role of $\mathrm{Na}_{\mathrm{v}} \mathrm{T} .9$ in neuropathic pain has not been established unequivocally so far. The channel is encoded by the $S C N 11 A$ gene ${ }^{38}$ and is next to DRG neurons, also expressed in trigeminal ganglion and myenteric neurons, ${ }^{58}$ and together with $\mathrm{Na}_{\mathrm{v}} 1.6, \mathrm{Na}_{\mathrm{v}} 1.7$ and $\mathrm{Na}_{\mathrm{v}} 1.8$ within free nerve terminals in skin and cornea. ${ }^{44}$ The kinetics of $\mathrm{Na}_{\mathrm{v}} 1.9$ implicate that it does not contribute to the action potential upstroke, but serves as a threshold channel. $59,60 \mathrm{~A}$ role in inflammatory and diabetic neuropathy pain has been shown for $\mathrm{Na}_{\mathrm{v}}$ 1.9. For example, in an inflamed hind paw, upregulation of $\mathrm{Na}_{\mathrm{v}}$. 9 has been demonstrated. ${ }^{61}$ In addition, a study with $\mathrm{Na}_{\mathrm{v}}$.9 knock-out mice showed that expression of $\mathrm{Na}_{\mathrm{v}} 1.9$ contributes to the persistent thermal hypersensitivity and spontaneous pain behaviour after peripheral inflammation. ${ }^{62}$ Increased $\mathrm{Na}_{\mathrm{v}}{ }^{1} .9$ levels have been found only in large diameter DRG neurons in painful diabetic neuropathy. ${ }^{63}$ According to these data, SCN11A may also be an appropriate candidate gene in I-SFN. In our SFN series, a group of $S C N g A_{\text {neg }}$ and $S C N 10 A_{\text {neg }}$ patients have demonstrated mutations in $S C N 11 A$ gene, and these findings are currently being evaluated to determine possible pathogenicity.

$\mathrm{Na} a_{\mathrm{v}} 1.6$

$\mathrm{Na}_{\mathrm{v}} 1.6$ is an important VGCS in the central nervous system and is encoded by the SCN8A gene..$^{8}$ This channel may play a role in SFN and other painful neuropathies, since it is also expressed in small and large-size DRG neurons and at the nodes of Ranvier and unmyelinated C-fibers. ${ }^{64,}{ }^{65}$ Accumulation of $\mathrm{Na}_{\mathrm{v}}{ }^{1.6}$ has been demonstrated in a damaged imfraorbital nerve of the rat. ${ }^{64}$ Besides, $\mathrm{Na}_{\mathrm{v}} \mathrm{r} .6$ plays a central role in mediating acute cooling-exacerbated symptoms following administration of the drug oxaliplatin. ${ }^{66}$ Moreover, considering the expression of the channels in the nodes of Ranvier, $\mathrm{Na}_{\mathrm{v}} \mathrm{\gamma} .6$ could be part of the pathophysiological mechanism in inflammatory neuropathies, since the lesions in these conditions are primary located at the nodes of Ranvier.

$\mathrm{Na} a_{v} \cdot 3$

Finally, $\mathrm{Na}_{\mathrm{v}} 1.3$, encoded by the $\mathrm{SCN}_{3} A$ gene,,$^{38}$ may also be of interest in I-SFN. $\mathrm{Na}_{\mathrm{v}} \mathrm{1} \cdot 3$ seems not only to be expressed in embryonic DRG, but may also be upregulated following axotomy. ${ }^{67}$ In addition, increased expression after nerve injury has been demonstrated in dorsal horn neurons ${ }^{68,69}$ and in supraspinal sensory neurons in the thalamus, ${ }^{70}$ which are all involved in pain signaling. 


\section{Other candidate genes}

Although the above mentioned VGSC genes all appear to be appropriate candidate genes for targeted sequencing in SFN, more pain-related genes have been described. ${ }^{11}$ Besides sodium channelopathies, also voltage gated potassium and calcium channelopathies have been associated with altered pain sensation. An amino acid-changing allele in the potassium channel KCNS 1 has been demonstrated to be a prognostic indicator of chronic pain risk. ${ }^{72}$ The calcium channel gene $\mathrm{CACNG}_{2}$ affects susceptibility to chronic pain following nerve injury. ${ }^{73}$ In addition, different members of the transient receptor potential (TRP) family of cation channels studied in animal models have demonstrated a role in neuropathic pain..$^{74,75}$

However, it is conceivable that pain-related genes exist that are not yet discovered. Another approach to reveal new candidate genes would be nextgeneration sequencing or whole exome sequencing. This technique allows unbiased identification of a genetic defect. It is an efficient strategy to selectively sequence the coding regions of the genome as a cheaper, but still effective alternative to whole genome sequencing. ${ }^{76-78}$ With this approach, several new loci in different non-neurological and neurological diseases have been identified. WES has already proven its value in particular movement disorders. ${ }^{76,78,79}$ Currently, neuropathies appear to be a suitable target for screening with WES. ${ }^{80}$

${ }^{87}$ For the patients in our cohort with an onset of symptoms $<40$ years or with affected family members, whole exome sequencing seems potentially useful when VCSC gene analyses are negative.

\section{Sodium channels and other pain conditions}

Painful peripheral neuropathies

SFN is a subtype of peripheral neuropathies. In this thesis only patients diagnosed with I-SFN have been examined, which is an even smaller and more specified subgroup. It would be interesting to investigate whether the results of this thesis can be extrapolated to patients with other (painful) peripheral neuropathies, which covers a large group of patients. Peripheral neuropathy in general affects approximately $2-3 \%$ of the world population; $88-91$ the prevalence increases with age, up to $10 \% .89,91-94$ With the global aging this will have important consequences on healthcare and social costs.

Approximately a quarter of the patients with diabetes mellitus suffer from painful neuropathy, 95,96 whereas the prevalence of cardiovascular autonomic neuropathy is around $20 \%$, increasing up to $65 \%$ with rising age and diabetes duration. ${ }^{97} \mathrm{~A}$ condition like diabetes seems not likely to be solely the cause of SFN. A potential genetic predisposition has also been suggested in diabetic painful neuropathies, since over more than half of the patients reported a family member with the same condition and complaints. ${ }^{98} \mathrm{It}$ is not far-fetched 
to hypothesize that VCSCs also may play a role in diabetic neuropathy, since changes of sodium channel expression have been described in diabetes. ${ }^{55}, 63,99$, 100 Moreover, recently a $\mathrm{Na}_{\mathrm{v}} 1.8$ mutation has been demonstrated in a diabetes type 1 patient with predominantly SFN..$^{57}$

In patients with cancer, chemotherapy-induced neuropathy is a debilitating and often dose-limiting side effect. ${ }^{101,} 102$ However, like in diabetes mellitus, not every patient will develop a neuropathy after treatment with chemotherapy. Depending on the dosage, it may occur in up to $50 \%$ of patients. ${ }^{103}$ Although the exact pathophysiological mechanism is unknown, there is some evidence for sodium channel dysfunction in this type of neuropathy. ${ }^{104,105}$

Therefore, it can be speculated that VGSC mutations might be a risk factor for the development of neuropathy, in which the associated conditions may function as a "traumatic" trigger. Hence, it seems logical to investigate the role of VGSC in the pathophysiology of other peripheral neuropathies. In diabetes, a large group of patients may possibly benefit from this approach, since its prevalence is expected to increase from 371 million individuals in 2012 to 552 million by the year 2030. ${ }^{106}$ In patients treated with chemotherapy, the presence of a pathogenic sodium channel mutation might increase the risk for developing painful neuropathy, and thereby may lead to adaptations of the treatment regimen.

\section{Other neuropathic pain conditions}

Trigeminal neuralgia is defined by the IASP as 'sudden, usually unilateral, severe, brief, stabbing, recurrent episodes of pain in the distribution of one or more branches of the trigeminal nerve.' 107 The condition can be subdivided into classical trigeminal neuralgia and symptomatic trigeminal neuralgia, in which the latter is caused by a demonstrable structural lesion other than vascular compression. ${ }^{108}$ The clinical pattern of trigeminal neuralgia shows similarities with the symptomatology of PEPD. Typical for both are the facial pain attacks evoked by non-noxious stimuli. ${ }^{108,} 109$ In our cohort, two patients diagnosed with $S C N_{9} A$ related SFN, had complaints of severe facial pain., ${ }^{2,6}$ The $^{N{ }_{\mathrm{v}} 1.7}$ mutation seen in these patients (I228M) demonstrated hyperexcitability in both DRG neurons and in trigeminal neurons. ${ }^{6}$ In addition, it is notable that the sodium channel blockers carbamazepine and oxcarbazepine are recommended as firstline medications in the current guidelines for trigeminal neuralgia treatment. ${ }^{110}$ These observations may suggest a possible pathophysiological relationship with sodium channels in trigeminal neuralgia. ${ }^{10}$ Recently, supporting evidence for this hypothesis has been demonstrated in a study in which the expression of $\mathrm{Na}_{\mathrm{v}}{ }^{1.3}, \mathrm{Na}_{\mathrm{v}} \mathrm{r} .7$ and $\mathrm{Na}_{\mathrm{v}} 1.8$ in the trigeminal nerve of trigeminal neuralgia patients was investigated." ${ }^{11} \mathrm{~A}$ reduction in the expression of $\mathrm{Na}_{\mathrm{v}} 1.7$, and an increase in the expression of $\mathrm{Na}_{\mathrm{v}} \mathrm{\gamma} .3$ compared to controls were demonstrated. As a result, 
studies on VGSC mutations in this group of patients may be considered. A special subtype of trigeminal neuralgia is burning mouth syndrome. ${ }^{122}$ Actually, this syndrome appears to be a trigeminal small fiber neuropathy. ${ }^{13}$ Tongue biopsies of patients showed a significantly lower density of epithelial nerve fibers than controls. ${ }^{113}$ The role of $\mathrm{Na}_{\mathrm{v}} 1.7$ in burning mouth syndrome has been investigated, but no significant alterations of expression could be demonstrated in a group of 7 patients. ${ }^{114}$ However, the $S C N_{9} A$ gene has never been screened for mutations in this condition. Also, in classical trigeminal neuralgia and burning mouth syndrome a role for other types of channelopathies, like TRPV 1 have been suggested. ${ }^{115,116}$

\section{Possible peripheral neuropathic pain condition}

In some pain conditions, like complex regional pain syndrome type I (CRPS-I) and fibromyalgia, evidence for a lesion or primary disease of the somatosensory system is not established.

Findings in CRPS-I like diffuse pain, skin hypersensitivity, microvascular degeneration and inflammatory changes, edema, abnormal sweating, skin and bone abnormalities, and reduced IENFD, have been linked to SFN. ${ }^{117-121}$ CRPS-I has also been addressed having a clinical overlap with IEM. Based on this, in a recent paper four familial cases of CRPS-I underwent $S C N_{9} A$ analysis, but no mutation was found in the $S C N_{9} A$ gene. However, no study has systematically examined whether a proportion of patients with CRPS-I may have a SCNgA mutation.

Fibromyalgia is often considered a functional disorder, but also central and peripheral nervous system involvement as part of the pathophysiology has been described. ${ }^{122-126}$ In our study cohort, some I-SFN patients were initially carrying the diagnosis of fibromyalgia before visiting our department. Recently, a study in 25 fibromyalgia patients confirmed small nerve fiber dysfunction in this condition with QST, skin biopsy and pain-related evoked potentials being abnormal. ${ }^{127}$ Previously, a link with SCNgA pathology has also been reported..$^{128}$ In a study among 73 Mexican women suffering from fibromyalgia, SCN9A analysis was performed, and an association with the rs6754031 polymorphism was found. ${ }^{128}$ These findings give rise to the thought that there might be a subgroup of patients diagnosed with fibromyalgia, who in fact have small fiber neuropathy. More structured research in larger groups of fibromyalgia patients is needed to unravel whether fibromyalgia belongs to the spectrum of small fiber neuropathy and whether VGSC mutations may play a role in its pathophysiology.

\section{Treatment}

The general aim of this thesis was to bring novel insights into the pathophysiology of SFN, with the final aim to create better therapeutic options. Neuropathic pain 
is one of the main symptoms of SFN. Worldwide neuropathic pain is estimated to affect $6-8 \%$ of the general population. ${ }^{129}$ It is an independent contributor to quality-of-life impairment in patients. ${ }^{130-132}$ Current treatments for painful neuropathies are largely inadequate. Less than $50 \%$ of patients achieve $50 \%$ of pain relief with commercially available drugs. This disappointing effect in painful neuropathies is due to the lack of drugs acting on target sites for which there is strong evidence of pathogenicity and the inability to identify responder patients. Elucidating the presence of $\mathrm{Na}_{\mathrm{v}} \mathrm{\gamma} .7$ mutations in SFN raises the question which drug should be considered as potentially appropriate to target SFN. However, the perfect sodium channel blocker will have to be developed.

As long as sixty years ago, it was demonstrated that the anticonvulsants phenytoin and carbamazepine could provide pain relief in trigeminal neuralgia. ${ }^{133}$ Subsequently, intravenous administration of the local anesthetic lidocaine proved also to be effective in this condition. ${ }^{134}$ Lidocaine also showed pain reduction in postoperative pain. ${ }^{35}$ The clinical utility of these drugs, however, is limited by side effects such as sedation, confusion, ataxia and diplopia which limit the dosages at which they can be given.

In the current guidelines on the pharmacological treatment of neuropathic pain, tricyclic antidepressants and serotonin-norepinephrine reuptake inhibitors are recommended as first-line treatment. ${ }^{136}$ However, only the first group has a sodium channel blocking effect in the range of therapeutic plasma concentrations. ${ }^{137}$ Amitriptyline is the oldest antidepressant drug with nonspecific sodium channel blocking activity. In the last decades, none of the new drugs, which have entered the market, have proven to be more effective than amitriptyline. ${ }^{36}$ In clinical practice the use of amitriptyline is limited by systemic anticholinergic side effects, particularly in elderly patients.

In IEM and PEPD patients carrying SCNgA mutations, the above-mentioned drugs are also frequently prescribed. Successful treatment with carbamazepine has been reported in PEPD, which could be explained by the characteristics of the drug. ${ }^{109,} 138,139$ Carbamazepine is a state-dependent sodium channel blocker that acts on the inactivated state of the channel. ${ }^{140}$ The impaired fastinactivation and persistent current in PEPD mutations would be tackled by the drug. ${ }^{109}$ In IEM, an inferior effect of carbamazepine is seen, because the drug does not act on the lowered activation thresholds that are the cause of the pain in IEM. However, in a family with IEM harboring mutation V400M, carbamazepine was successful, probably due to the specific localization of the mutation, which is close to the local anesthetic binding region. ${ }^{141}$ In another IEM patient, the lidocaine derivate mexiletine caused pain relief, possibly because of the enhanced lidocaine use-dependent block of the channel. ${ }^{142}$

However, none of these drugs are sodium channel-isoform specific, which probably explains their cardiovascular and central nervous system adverse 
events. ${ }^{143}, 144$ These drugs in turn have demonstrated some therapeutic window, which could be an explanation for the partial pain relief that generally is seen in SFN.

\section{Development of targeted treatment}

In order to avoid central nervous system and cardiac side effects, selective $\mathrm{Na}_{\mathrm{v}} \mathrm{T} .7$ blockers would be the ideal treatment, since in humans the channel has mainly been demonstrated in the peripheral and autonomic nervous system. ${ }^{38}$ Animal studies have shown efficacy of some potential $\mathrm{Na}_{\mathrm{v}} 1.7$ selective sodium channel blockers..$^{145-148}$ However, the efficacy and safety in humans remains to be demonstrated.

Another possible therapeutic modality would be the relatively new sodium channel blocker lacosamide, which has been registered for treating epilepsy. Lacosamide displays a unique mechanism of action in that it seemingly selectively stabilizes channels into the slow- inactivated state. ${ }^{149}$ It inhibits currents from $\mathrm{Na}_{\mathrm{v}} \mathrm{T} .3, \mathrm{Na}_{\mathrm{v}} \mathrm{1} .7$, and $\mathrm{Na}_{\mathrm{v}} 1.8$, but only after prolonged depolarisations, consistent with an enhancement in slow-inactivation with no effect on fast-inactivation. ${ }^{150}$ Since most of the demonstrated $\mathrm{Na}_{\mathrm{v}} \mathrm{T} .7$ mutations in SFN showed impaired slow-inactivation, pain relief in patients with $S C N g A$ mutations could be expected. ${ }^{2}$ Future studies are needed to determine the effect of this potentially specific drug in patients with SCNgA mutations-related SFN.

\section{Conclusions}

In this thesis, $\mathrm{Na}_{\mathrm{v}} \mathrm{T} .7$ mutations in I-SFN have been demonstrated providing patients with more certainty regarding the origin of their symptoms. The findings in this thesis contribute to a different thinking in chronic neuropathic pain, which may open new doors for translational research and create more new possibilities for therapeutic developments.

Through functional analyses the effects of the mutations on channel function and DRG neuron firing properties have been shown. These results explain the clinical picture of pain and autonomic dysfunction in selected SFN patients. There is evidence that the mutations contribute to IENF loss, explaining one of the diagnostic criteria of SFN. With the rapid pace of identification of new mutations, more efficient techniques are needed to analyze the pathogenicity of the mutations. Zebrafish models may be a good additional tool to the voltage clamp and current clamp studies and may be useful in testing the effect and toxicity of developmental treatment. Additional research is needed to unravel which other genes are involved in the pathophysiology of SFN. It can be of great interest to investigate possible contribution of sodium channel mutations 
in other neuropathies, such as painful diabetic neuropathy or chemotherapy induced neuropathy, or other neuropathic pain conditions or unexplained pain conditions with neuropathic features. The increasing knowledge regarding the underlying pathophysiology in SFN provides a clear target on which drugs should act for optimal treatment.

In conclusion, the discovery of sodium channel mutations in I-SFN has brought a new vision on neuropathic pain syndromes and peripheral neuropathies, wrinkling our thoughts to a more "out of the box" way of looking at painful conditions. 


\section{References}

1. Hoeijmakers, J.G., Faber, C.G., Lauria, G., Merkies, I.S. \& Waxman, S.G. Small-fibre neuropathies-advances in diagnosis, pathophysiology and management. Nat Rev Neurol 8, 369-79 (2012).

2. Faber, C.G. et al. Gain of function Nanu1.7 mutations in idiopathic small fiber neuropathy. Ann Neurol 7 , 26-39 (2012).

3. Asch, D.A., Patton, J.P. \& Hershey, J.C. Knowing for the sake of knowing: the value of prognostic information. Med Decis Making 10, 47-57 (1990).

4. Williams de, A.C. \& Cella, M. Medically unexplained symptoms and pain: misunderstanding and myth. Curr Opin Support Palliat Care 6, 201-6 (2012).

5. Tait, R.C., Chibnall, J.T. \& Kalauokalani, D. Provider judgments of patients in pain: seeking symptom certainty. Pain Med 10, 11-34 (2009).

6. Estacion, M. et al. Intra- and interfamily phenotypic diversity in pain syndromes associated with a gain of-function variant of $\mathrm{NaV}$ 1.7. Mol Pain 7, 92 (2011)

7. Hoeijmakers, J.G. et al. Small nerve fibres, small hands and small feet: a new syndrome of pain, dysautonomia and acromesomelia in a kindred with a novel $\mathrm{NaV}_{1}$.7 mutation. Brain 135, 345-58 (2012).

8. Han, C. et al. Nav1.7-related small fiber neuropathy: impaired slow-inactivation and DRG neuron hyperexcitability. Neurology 78, 1635-43 (2012).

9. Han, C. et al. Functional profiles of $\mathrm{SCN}_{9} \mathrm{~A}$ variants in dorsal root ganglion neurons and superior cervical ganglion neurons correlate with autonomic symptoms in small fibre neuropathy. Brain 135, 2613-28 (2012).

10. Hoeijmakers, J.G., Merkies, I.S., Gerrits, M.M., Waxman, S.G. \& Faber, C.G. Genetic aspects of sodium channelopathy in small fiber neuropathy. Clin Genet 82, 351-8 (2012).

11. Choi, J.S. et al. Alternative splicing may contribute to time-dependent manifestation of inherited erythromelalgia. Brain 133, 1823-35 (2010).

12. Dabby, R. et al. Chronic non-paroxysmal neuropathic pain - Novel phenotype of mutation in the sodium channel SCNgA gene.J Neurol Sci 301, 90-2 (2011).

13. Cregg, R. et al. Novel Mutations Mapping to the Fourth Sodium Channel Domain of Nav1.7 Result in Variable Clinical Manifestations of Primary Erythromelalgia. Neuromolecular Med (2013).

14. Estacion, M. et al. $\mathrm{NaV}_{1} .7$ gain-of-function mutations as a continuum: A1632E displays physiological changes associated with erythromelalgia and paroxysmal extreme pain disorder mutations and produces symptoms of both disorders. J Neurosci 28, 11079-88 (2008).

15. Wilde, A.A. \& Brugada, R. Phenotypical manifestations of mutations in the genes encoding subunits of the cardiac sodium channel. Circ Res 108, 884-97 (2011).

16. Guerrini, R. et al. Variable epilepsy phenotypes associated with a familial intragenic deletion of the $S_{C N} N_{1}$ gene. Epilepsia 51, 2474-7 (2010).

17. Estacion, M. et al. A sodium channel gene $\mathrm{SCNgA}$ polymorphism that increases nociceptor excitability. Ann Neurol 66, 862-6 (2009).

18. Reimann, F. et al. Pain perception is altered by a nucleotide polymorphism in SCNgA. Proc Natl Acad Sci US A 107, 5148-53 (2010).

19. Waxman, S.G. Polymorphisms in ion channel genes: emerging roles in pain. Brain 133, 2515-8 (2010).

20. Diatchenko, L. et al. Genetic basis for individual variations in pain perception and the development of a chronic pain condition. Hum Mol Genet 14, 135-43 (2005).

21. Tegeder, I. et al. GTP cyclohydrolase and tetrahydrobiopterin regulate pain sensitivity and persistence. Nat Med 12, 1269-77 (2006).

22. Howell, V.M. et al. Evidence for a direct role of the disease modifier $\mathrm{SCNM}_{1}$ in splicing. Hum Mol Genet 16, 2506-16 (2007)

23. Rush, A.M. et al. Differential modulation of sodium channel $\mathrm{Na}(\mathrm{v}) 1.6$ by two members of the fibroblast growth factor homologous factor 2 subfamily. Eur J Neurosci 23, 2551-62 (2006).

24. Wittmack, E.K. et al. Fibroblast growth factor homologous factor 2B: association with Nav1.6 and selective colocalization at nodes of Ranvier of dorsal root axons. J Neurosci 24, 6765-75 (2004).

25. Seo, S. et al. Epigenetics: A Promising Paradigm for Better Understanding and Managing Pain. J Pain (2013).

26. Sibille, K.T., Witek-Janusek, L., Mathews, H.L. \& Fillingim, R.B. Telomeres and epigenetics: potential relevance to chronic pain. Pain 153, 1789-93 (2012). 
27. Denk, F. \& McMahon, S.B. Chronic pain: emerging evidence for the involvement of epigenetics. Neuron 73, 435-44 (2012).

28. Persson, A.K. et al. Sodium-calcium exchanger and multiple sodium channel isoforms in intra-epidermal nerve terminals. Mol Pain 6, 84 (2010).

29. Lehning, E.J., Doshi, R., Isaksson, N., Stys, P.K. \& LoPachin, R.M., Jr. Mechanisms of injury-induced calcium entry into peripheral nerve myelinated axons: role of reverse sodium-calcium exchange. $J$ Neurochem 66, 493-500 (1996).

30. Petrescu, N., Micu, I., Malek, S., Ouardouz, M. \& Stys, P.K. Sources of axonal calcium loading during in vitro ischemia of rat dorsal roots. Muscle Nerve 35, 451-7 (2007).

31. Ransom, B.R. \& Philbin, D.M., Jr. Anoxia-induced extracellular ionic changes in CNS white matter: the role of glial cells. Can J Physiol Pharmacol 70 Suppl, S181-9 (1992).

32. Persson, A.K. et al. Neuropathy-associated $\mathrm{Na}(\mathrm{V}) 1.7$ variant $1228 \mathrm{M}$ impairs integrity of dorsal root ganglion neuron axons. Ann Neurol 73, 140-5 (2013).

33. Howe, K. et al. The zebrafish reference genome sequence and its relationship to the human genome. Nature 496, 498-503 (2013).

34. Lieschke, G.J. \& Currie, P.D. Animal models of human disease: zebrafish swim into view. Nat Rev Genet 8, 353-67 (2007).

35. Novak, A.E. et al. Gene duplications and evolution of vertebrate voltage-gated sodium channels. J Mol Evol 63, 208-21 (2006).

36. Prober, D.A. et al. Zebrafish TRPA channels are required for chemosensation but not for thermosensation or mechanosensory hair cell function.J Neurosci 28, 10102-10 (2008).

37. Khan, T.M. et al. Vincristine and bortezomib cause axon outgrowth and behavioral defects in larval zebrafish. J Peripher Nerv Syst 17, 76-89 (2012).

38. Catterall, W.A., Goldin, A.L. \& Waxman, S.G. International Union of Pharmacology. XLVII. Nomenclature and structure-function relationships of voltage-gated sodium channels. Pharmacol Rev 57, 397-409 (2005).

39. Renganathan, M., Cummins, T.R. \& Waxman, S.G. Contribution of $\mathrm{Na}(\mathrm{v}) 1.8$ sodium channels to action potential electrogenesis in DRG neurons. J Neurophysiol 86, 629-40 (2001).

40. Blair, N.T. \& Bean, B.P. Roles of tetrodotoxin (TTX)-sensitive Na+ current, TTX-resistant $\mathrm{Na}+$ current, and $\mathrm{Ca2}+$ current in the action potentials of nociceptive sensory neurons. J Neurosci 22, 10277-90 (2002).

41. Akopian, A.N., Sivilotti, L. \& Wood, J.N. A tetrodotoxin-resistant voltage-gated sodium channel expressed by sensory neurons. Nature 379, 257-62 (1996).

42. Rush, A.M. et al. Contactin regulates the current density and axonal expression of tetrodotoxin-resistant but not tetrodotoxin-sensitive sodium channels in DRG neurons. Eur J Neurosci 22, 39-49 (2005).

43. Zhao, P. et al. Voltage-gated sodium channel expression in rat and human epidermal keratinocytes: evidence for a role in pain. Pain 139, 90-105 (2008).

44. Black, J.A. \& Waxman, S.G. Molecular identities of two tetrodotoxin-resistant sodium channels in corneal axons. Exp Eye Res 75, 193-9 (2002)

45. Rush, A.M. et al. A single sodium channel mutation produces hyper- or hypoexcitability in different types of neurons. Proc Natl Acad Sci U S A 103, 8245-50 (2006).

46. Choi, J.S. \& Waxman, S.G. Physiological interactions between $\mathrm{Na}(\mathrm{v}) 1.7$ and $\mathrm{Na}(\mathrm{v}) 1.8$ sodium channels: a computer simulation study. J Neurophysiol 106, 3173-84 (2011).

47. Akopian, A.N. et al. The tetrodotoxin-resistant sodium channel SNS has a specialized function in pain pathways. Nat Neurosci 2, 541-8 (1999).

48. Zimmermann, K. et al. Sensory neuron sodium channel Nav1.8 is essential for pain at low temperatures. Nature 447, 855-8 (2007)

49. Abrahamsen, B. et al. The cell and molecular basis of mechanical, cold, and inflammatory pain. Science 321, 702-5 (2008).

50. Lai, J. et al. Inhibition of neuropathic pain by decreased expression of the tetrodotoxin-resistant sodium channel, NaV1.8. Pain 95, 143-52 (2002).

51. Gold, M.S. et al. Redistribution of $\mathrm{Na}(\mathrm{V}) 1.8$ in uninjured axons enables neuropathic pain. J Neurosci 23 , 158-66 (2003).

52. Novakovic, S.D. et al. Distribution of the tetrodotoxin-resistant sodium channel $\mathrm{PN}_{3}$ in rat sensory neurons in normal and neuropathic conditions. J Neurosci 18, 2174-87 (1998).

53. Coward, K. et al. Immunolocalization of SNS/PN 3 and $\mathrm{NaN}_{\mathrm{S}} \mathrm{SNS} 2$ sodium channels in human pain states. Pain 85, 41-50 (2000). 
54. Kretschmer, $\mathrm{T}$. et al. Accumulation of $\mathrm{PN}_{1}$ and $\mathrm{PN}_{3}$ sodium channels in painful human neuroma-evidence from immunocytochemistry. Acta Neurochir (Wien) 144, 803-10; discussion 810 (2002).

55. Bierhaus, A. et al. Methylglyoxal modification of Nav1.8 facilitates nociceptive neuron firing and causes hyperalgesia in diabetic neuropathy. Nat Med 18, 926-33 (2012).

56. Mert, T. \& Gunes, Y. Antinociceptive activities of lidocaine and the nav1.8 blocker a803467 in diabetic rats. J Am Assoc Lab Anim Sci 51, 579-85 (2012).

57. Faber, C.G. et al. Gain-of-function Nav1.8 mutations in painful neuropathy. Proc Natl Acad Sci U S A 109, 19444-9 (2012).

58. Dib-Hajj, S., Black, J.A., Cummins, T.R. \& Waxman, S.G. NaN/Navı.9: a sodium channel with unique properties. Trends Neurosci 25, 253-9 (2002)

59. Herzog, R.I., Cummins, T.R. \& Waxman, S.G. Persistent TTX-resistant Na+ current affects resting potential and response to depolarization in simulated spinal sensory neurons.J Neurophysiol 86, 1351-64 (2001).

6o. Copel, C. et al. Activation of neurokinin 3 receptor increases $\mathrm{Na}(\mathrm{v}) 1.9$ current in enteric neurons.J Physiol 587, 1461-79 (2009).

61. Tate, S. et al. Two sodium channels contribute to the TTX-R sodium current in primary sensory neurons. Nat Neurosci 1, 653-5 (1998).

62. Priest, B.T. et al. Contribution of the tetrodotoxin-resistant voltage-gated sodium channel NaV1.9 to sensory transmission and nociceptive behavior. Proc Natl Acad Sci U S A 102, 9382-7 (2005).

63. Craner, M.J., Klein, J.P., Renganathan, M., Black, J.A. \& Waxman, S.G. Changes of sodium channel expression in experimental painful diabetic neuropathy. Ann Neurol 52, 786-92 (2002).

64. Henry, M.A., Freking, A.R., Johnson, L.R. \& Levinson, S.R. Sodium channel Nav1.6 accumulates at the site of infraorbital nerve injury. BMC Neurosci 8, 56 (2007).

65. Gasser, A. et al. An ankyrinG-binding motif is necessary and sufficient for targeting Nav1.6 sodium channels to axon initial segments and nodes of Ranvier. J Neurosci 32, 7232-43 (2012).

66. Sittl, R. et al. Anticancer drug oxaliplatin induces acute cooling-aggravated neuropathy via sodium channel subtype $\mathrm{Na}(\mathrm{V}) 1.6$-resurgent and persistent current. Proc Natl Acad Sci U S A 109, 6704-9 (2012).

67. Waxman, S.G., Kocsis, J.D. \& Black, J.A. Type III sodium channel mRNA is expressed in embryonic but not adult spinal sensory neurons, and is reexpressed following axotomy.J Neurophysiol 72, 466-70 (1994).

68. Hains, B.C., Saab, C.Y., Klein, J.P., Craner, M.J. \& Waxman, S.G. Altered sodium channel expression in second-order spinal sensory neurons contributes to pain after peripheral nerve injury. J Neurosci $\mathbf{2 4}, 4832$ 9 (2004).

69. Lampert, A., Hains, B.C. \& Waxman, S.G. Upregulation of persistent and ramp sodium current in dorsal horn neurons after spinal cord injury. Exp Brain Res 174, 660-6 (2006).

70. Zhao, P., Waxman, S.G. \& Hains, B.C. Sodium channel expression in the ventral posterolateral nucleus of the thalamus after peripheral nerve injury. Mol Pain 2, 27 (2006).

71. Young, E.E., Lariviere, W.R. \& Belfer, I. Genetic basis of pain variability: recent advances. J Med Genet 49, 1-9 (2012).

72. Costigan, M. et al. Multiple chronic pain states are associated with a common amino acid-changing allele in KCNS1. Brain 133, 2519-27 (2010).

73. Nissenbaum, J. et al. Susceptibility to chronic pain following nerve injury is genetically affected by CACNG2. Genome Res 20, 1180-90 (2010).

74. Cregg, R., Momin, A., Rugiero, F., Wood, J.N. \& Zhao, J. Pain channelopathies. J Physiol 588, 1897-904 (2010).

75. Alessandri-Haber, N. et al. Transient receptor potential vanilloid 4 is essential in chemotherapy-induced neuropathic pain in the rat. J Neurosci 24, 4444-52 (2004).

76. Bras, J., Guerreiro, R. \& Hardy, J. Use of next-generation sequencing and other whole-genome strategies to dissect neurological disease. Nat Rev Neurosci 13, 453-64 (2012).

77. Do, R., Kathiresan, S. \& Abecasis, G.R. Exome sequencing and complex disease: practical aspects of rare variant association studies. Hum Mol Genet 21, R1-9 (2012).

78. Pittman, A. \& Hardy, J. Genetic Analysis in Neurology: The Next 10 Years. JAMA Neurol, 1-7 (2013).

79. Gerards, M. et al. Exome sequencing reveals a novel Moroccan founder mutation in SLC19A3 as a new cause of early-childhood fatal Leigh syndrome. Brain 136, 882-90 (2013).

80. Kennerson, M.L. et al. A new locus for X-linked dominant Charcot-Marie-Tooth disease (CMTX6) is caused by mutations in the pyruvate dehydrogenase kinase isoenzyme 3 (PDK3) gene. Hum Mol Genet 22, 1404-16 (2013). 
81. Ylikallio, E. et al. Deficiency of the $\mathrm{E}_{3}$ ubiquitin ligase TRIM2 in early-onset axonal neuropathy. Hum $\mathrm{Mol}$ Genet (2013).

82. Lee, S.S. et al. Proximal Dominant Hereditary Motor and Sensory Neuropathy With Proximal Dominance Association With Mutation in the TRK-Fused Gene. JAMA Neurol, 1-9 (2013).

83. Auranen, M. et al. Dominant GDAP1 founder mutation is a common cause of axonal Charcot-MarieTooth disease in Finland. Neurogenetics (2013).

84. Lee, H.J. et al. Two novel mutations of GARS in Korean families with distal hereditary motor neuropathy type V.J Peripher Nerv Syst 17, 418-21 (2012).

85. Choi, B.O. et al. Clinical and histopathological study of Charcot-Marie-Tooth neuropathy with a novel SgoW mutation in BSCL2. Neurogenetics 14, 35-42 (2013).

86. Pyle, A. et al. Prominent sensorimotor neuropathy due to SACS mutations revealed by whole-exome sequencing. Arch Neurol 69, 1351-4 (2012).

87. Beetz, C. et al. Exome sequencing identifies a REEP1 mutation involved in distal hereditary motor neuropathy type V. Am J Hum Genet 91, 139-45 (2012).

88. Martyn, C.N. \& Hughes, R.A. Epidemiology of peripheral neuropathy. J Neurol Neurosurg Psychiatry 62 , 310-8 (1997).

89. England, J.D. \& Asbury, A.K. Peripheral neuropathy. Lancet 363, 2151-61 (2004).

90. Gregg, E.W. et al. Prevalence of lower-extremity disease in the US adult population $>=40$ years of age with and without diabetes: 1999-2000 national health and nutrition examination survey. Diabetes Care 27, 1591-7 (2004).

91. Mold, J.W., Vesely, S.K., Keyl, B.A., Schenk, J.B.\& Roberts, M. The prevalence, predictors, and consequences of peripheral sensory neuropathy in older patients. J Am Board Fam Pract 17, $309-18$ (2004)

92. Beghi, E. \& Monticelli, M.L. Chronic symmetric symptomatic polyneuropathy in the elderly: a field screening investigation of risk factors for polyneuropathy in two Italian communities. Italian General Practitioner Study Group (IGPST). J Clin Epidemiol 51, 697-702 (1998).

93. Repetto, L. Greater risks of chemotherapy toxicity in elderly patients with cancer. J Support Oncol 1, 18-24 (2003).

94. Baldereschi, M. et al. Epidemiology of distal symmetrical neuropathies in the Italian elderly. Neurology 68 , 1460-7 (2007).

95. Davies, M., Brophy, S., Williams, R. \& Taylor, A. The prevalence, severity, and impact of painful diabetic peripheral neuropathy in type 2 diabetes. Diabetes Care 29, 1518-22 (2006).

96. Abbott, C.A., Malik, R.A., van Ross, E.R., Kulkarni, J. \& Boulton, A.J. Prevalence and characteristics of painful diabetic neuropathy in a large community-based diabetic population in the U.K. Diabetes Care 34, 2220-4 (2011)

97. Spallone, V. et al. Cardiovascular autonomic neuropathy in diabetes: clinical impact, assessment, diagnosis, and management. Diabetes Metab Res Rev (2011).

98. Galer, B.S., Gianas, A. \& Jensen, M.P. Painful diabetic polyneuropathy: epidemiology, pain description, and quality of life. Diabetes Res Clin Pract 47, 123-8 (2000).

99. Hong, S., Morrow, T.J., Paulson, P.E., Isom, L.L. \& Wiley, J.W. Early painful diabetic neuropathy is associated with differential changes in tetrodotoxin-sensitive and -resistant sodium channels in dorsal root ganglion neurons in the rat. J Biol Chem 279, 29341-50 (2004).

100. Hirade, M., Yasuda, H., Omatsu-Kanbe, M., Kikkawa, R. \& Kitasato, H. Tetrodotoxin-resistant sodium channels of dorsal root ganglion neurons are readily activated in diabetic rats. Neuroscience 90, 933-9 (1999).

101. Markman, M. Chemotherapy-associated neurotoxicity: an important side effect-impacting on quality, rather than quantity, of life. J Cancer Res Clin Oncol 122, 511-2 (1996).

102. Cavaletti, G., Alberti, P., Frigeni, B., Piatti, M. \& Susani, E. Chemotherapy-induced neuropathy. Curr Treat Options Neurol 13, 180-90 (2011).

103. Kautio, A.L., Haanpaa, M., Kautiainen, H., Kalso, E. \& Saarto, T. Burden of chemotherapy-induced neuropathy--a cross-sectional study. Support Care Cancer 19, 1991-6 (2011).

104. Park, S.B. et al. Oxaliplatin-induced neurotoxicity: changes in axonal excitability precede development of neuropathy. Brain 132, 2712-23 (2009).

105. Krishnan, A.V., Goldstein, D., Friedlander, M. \& Kiernan, M.C. Oxaliplatin and axonal Na+ channel function in vivo. Clin Cancer Res 12, 4481-4 (2006).

106. http://www.idf.org/diabetesatlas/5e/Update2012. 
107. Merskey, H. \& Bogduk, N. Classification of Chronic Pain. Descriptors of Chronic Pain Syndromes and Definitions of Pain Terms. (Seattle: IASP Press, 1994).

108. Rosenbaum, T., Gordon-Shaag, A., Munari, M. \& Gordon, S.E. Ca2+/calmodulin modulates TRPV1 activation by capsaicin. J Gen Physiol 123, 53-62 (2004).

109. Fertleman, C.R. et al. SCNgA mutations in paroxysmal extreme pain disorder: allelic variants underlie distinct channel defects and phenotypes. Neuron 52, 767-74 (2006).

110. Cruccu, G. et al. AAN-EFNS guidelines on trigeminal neuralgia management. Eur J Neurol 15, 1013-28 (2008)

111. Siqueira, S.R., Alves, B., Malpartida, H.M., Teixeira, M.J. \& Siqueira, J.T. Abnormal expression of voltagegated sodium channels Nav1.7, Nav1.3 and Nav1.8 in trigeminal neuralgia. Neuroscience 164, 573-7 (2009).

112. Jaaskelainen, S.K. Pathophysiology of primary burning mouth syndrome. Clin Neurophysiol 123, 71-7 (2012).

113. Lauria, G. et al. Trigeminal small-fiber sensory neuropathy causes burning mouth syndrome. Pain 115 332-7 (2005).

114. Beneng, K., Renton, T., Yilmaz, Z., Yiangou, Y. \& Anand, P. Sodium channel Na v 1.7 immunoreactivity in painful human dental pulp and burning mouth syndrome. BMC Neurosci 11, 71 (2010).

115. Urano, H., Ara, T., Fujinami, Y. \& Hiraoka, B.Y. Aberrant TRPV 1 expression in heat hyperalgesia associated with trigeminal neuropathic pain. Int J Med Sci 9, 690-7 (2012).

116. Yilmaz, Z. et al. Burning mouth syndrome as a trigeminal small fibre neuropathy: Increased heat and capsaicin receptor TRPV in nerve fibres correlates with pain score. J Clin Neurosci 14, 864-71 (2007).

117. Oaklander, A.L. \& Fields, H.L. Is reflex sympathetic dystrophy/complex regional pain syndrome type I a small-fiber neuropathy? Ann Neurol 65, 629-38 (2009).

118. Oaklander, A.L. et al. Evidence of focal small-fiber axonal degeneration in complex regional pain syndrome-I (reflex sympathetic dystrophy). Pain 120, 235-43 (2006).

119. Chemali, K.R. \& Zhou, L. Small fiber degeneration in post-stroke complex regional pain syndrome I. Neurology 69, 316-7 (2007)

120. de Mos, M., Sturkenboom, M.C. \& Huygen, F.J. Current understandings on complex regional pain syndrome. Pain Pract 9, 86-99 (2009).

121. Maihofner, C., Seifert, F. \& Markovic, K. Complex regional pain syndromes: new pathophysiological concepts and therapies. EurJ Neurol 17, 649-60 (2010).

122. Klauenberg, S. et al. Depression and changed pain perception: hints for a central disinhibition mechanism. Pain 140, 332-43 (2008).

123. Staud, R. Brain imaging in fibromyalgia syndrome. Clin Exp Rheumatol 29, S109-17 (2011).

124. Hurtig, I.M., Raak, R.I., Kendall, S.A., Gerdle, B. \& Wahren, L.K. Quantitative sensory testing in fibromyalgia patients and in healthy subjects: identification of subgroups. Clin J Pain 17, 316-22 (2001).

125. Blumenstiel, K. et al. Quantitative sensory testing profiles in chronic back pain are distinct from those in fibromyalgia. Clin J Pain 27, 682-90 (2011).

126. Gibson, S.J., Littlejohn, G.O., Gorman, M.M., Helme, R.D. \& Granges, G. Altered heat pain thresholds and cerebral event-related potentials following painful $\mathrm{CO}_{2}$ laser stimulation in subjects with fibromyalgia syndrome. Pain 58, 185-93 (1994).

127. Uceyler, N. et al. Small fibre pathology in patients with fibromyalgia syndrome. Brain (2013).

128. Vargas-Alarcon, G. et al. A SCNgA gene-encoded dorsal root ganglia sodium channel polymorphism associated with severe fibromyalgia. BMC Musculoskelet Disord 13, 23 (2012).

129. Bouhassira, D., Lanteri-Minet, M., Attal, N., Laurent, B. \& Touboul, C. Prevalence of chronic pain with neuropathic characteristics in the general population. Pain 136, 380-7 (2008)

130. Attal, N., Lanteri-Minet, M., Laurent, B., Fermanian, J. \& Bouhassira, D. The specific disease burden of neuropathic pain: results of a French nationwide survey. Pain 152, 2836-43 (2011).

131. Gormsen, L., Rosenberg, R., Bach, F.W. \& Jensen, T.S. Depression, anxiety, health-related quality of life and pain in patients with chronic fibromyalgia and neuropathic pain. Eur J Pain 14, 127 e1-8 (2010).

132. Bakkers, M., Faber, C.G., Hoeijmakers, J.G., Lauria, G. \& Merkies, I.S. Small fibers, large impact: quality of life in small fiber neuropathy. Muscle Nerve In press (2013).

133. Blom, S. Trigeminal neuralgia: its treatment with a new anticonvulsant drug (G-32883). Lancet 1, 839-40 (1962). 
134. Kugelberg, E. \& Lindblom, U. The mechanism of the pain in trigeminal neuralgia. J Neurol Neurosurg Psychiatry 22, 36-43 (1959).

135. Bartlett, E.E. \& Hutserani, O. Xylocaine for the relief of postoperative pain. Anesth Analg 40, 296-304 (1961).

136. Attal, N. et al. EFNS guidelines on the pharmacological treatment of neuropathic pain: 2010 revision. Eur J Neurol 17, 1113-e88 (2010).

137. Dick, I.E. et al. Sodium channel blockade may contribute to the analgesic efficacy of antidepressants. J Pain 8, 315-24 (2007).

138. Ragsdale, D.S. \& Avoli, M. Sodium channels as molecular targets for antiepileptic drugs. Brain Res Brain Res Rev 26, 16-28 (1998).

139. Rush, A.M. \& Elliott, J.R. Phenytoin and carbamazepine: differential inhibition of sodium currents in small cells from adult rat dorsal root ganglia. Neurosci Lett 226, 95-8 (1997).

140. Song, J.H., Nagata, K., Huang, C.S., Yeh, J.Z. \& Narahashi, T. Differential block of two types of sodium channels by anticonvulsants. Neuroreport 7, 3031-6 (1996).

141. Fischer, T.Z. et al. A novel Nav1.7 mutation producing carbamazepine-responsive erythromelalgia. Ann Neurol 65, 733-41 (2009).

142. Choi, J.S. et al. Mexiletine-responsive erythromelalgia due to a new $\mathrm{Na}(\mathrm{v}) 1.7$ mutation showing usedependent current fall-off. Exp Neurol 216, 383-9 (2009).

143. Sindrup, S.H. \& Jensen, T.S. Are sodium channel blockers useless in peripheral neuropathic pain? Pain 128, 6-7 (2007).

144. Gerner, P. \& Strichartz, G.R. Sensory and motor complications of local anesthetics. Muscle Nerve 37, 421-5 (2008)

145. Chowdhury, S. et al. Discovery of XEN907, a spirooxindole blocker of $\mathrm{NaV}_{1.7}$ for the treatment of pain. Bioorg Med Chem Lett 21, 3676-81 (2011).

146. Bregman, $\mathrm{H}$. et al. Identification of a potent, state-dependent inhibitor of Nav1.7 with oral efficacy in the formalin model of persistent pain.J Med Chem 54, 4427-45 (2011).

147. London, C. et al. Imidazopyridines: a novel class of hNav1.7 channel blockers. Bioorg Med Chem Lett 18, 1696-701 (2008).

148. Williams, B.S. et al. Characterization of a new class of potent inhibitors of the voltage-gated sodium channel Nav1.7. Biochemistry 46, 14693-703 (2007).

149. Errington, A.C., Stohr, T., Heers, C. \& Lees, G. The investigational anticonvulsant lacosamide selectively enhances slow inactivation of voltage-gated sodium channels. Mol Pharmacol 73, 157-69 (2008).

150. Sheets, P.L., Heers, C., Stoehr, T. \& Cummins, T.R. Differential block of sensory neuronal voltage-gated sodium channels by lacosamide [(2R)-2-(acetylamino)-N-benzyl-3-methoxypropanamide], lidocaine, and carbamazepine. J Pharmacol Exp Ther 326, 89-99 (2008). 
Chapter

9. 

Chapter 10

Nederlandse samenvatting 


\section{Samenvatting}

Dunnevezelneuropathie (DVN) is een aandoening waarbij selectief of overwegend de dunne zenuwvezels ( $A \delta$ en $C$ ) zijn aangedaan. Het klinisch beeld wordt gekenmerkt door neuropathische pijn (pijn vanuit het zenuwstelsel) en autonome stoornissen; dit zijn stoornissen in automatisch verlopende functies zoals hartritme, zweetpatroon en problemen met urine en ontlasting (incontinentie, diarree, obstipatie). Bij neurologisch onderzoek worden vrijwel geen afwijkingen gevonden, behalve in het registreren van pijn en temperatuur. De diagnose wordt gesteld op basis van de symptomen, in combinatie met een verlaagd aantal intraepidermale zenuwvezels (zenuwuiteinden in de opperhuid) in het huidbiopt en/of een afwijkend temperatuurdrempelonderzoek. Nadat de diagnose DVN is gesteld moet er worden gezocht naar een onderliggende oorzaak, aangezien sommige van deze oorzaken behandelbaar zijn.

Er kunnen vele aandoeningen ten grondslag liggen aan DVN, maar in $24 \%$ tot 93\% van de gevallen kan er geen onderliggende oorzaak worden aangetoond. Bij deze groep patiënten bestaat de therapie uit symptoombestrijding, wat vaak onvoldoende werkzaam is. Om betere en meer doelgerichte therapieën te kunnen ontwikkelen, is het nodig het mechanisme voor het ontstaan van DVN (de pathofysiologie) te ontrafelen. Er zijn verschillende theorieën over de pathofysiologie van DVN. Opvallend is dat veel verschillende aandoeningen tot hetzelfde klinisch beeld bij DVN kunnen leiden. Een verklaring hiervoor zou kunnen zijn dat er sprake is van een 'final common pathway', oftewel een gezamenlijk mechanisme dat uiteindelijk tot min of meer eenzelfde klinisch beeld leidt. Er zijn steeds meer aanwijzingen voor een erfelijke onderliggende (genetische) oorzaak.

In dit proefschrift is op basis van dit achterliggende idee verder onderzoek gedaan naar een kandidaatgen: het SCNgA-gen, dat codeert voor het spanningsafhankelijke natriumkanaal $\mathrm{Na}_{\mathrm{v}}$ 1.7. Spanningsafhankelijke natriumkanalen zijn van belang voor het ontstaan en de geleiding van actiepotentialen in onder andere het zenuwstelsel. Bij de mens zijn $\mathrm{Na}_{\mathrm{v}} 1.6, \mathrm{Na}_{\mathrm{v}} 1.7, \mathrm{Na}_{\mathrm{v}} 1.8$ en $\mathrm{Na}_{\mathrm{v}} \mathrm{T} .9$ aanwezig in de cellichamen (dorsale ganglioncellen) van de dunne $A \delta$ vezels en C-vezels. Van $\mathrm{Na}_{\mathrm{v}} 1.7, \mathrm{Na}_{\mathrm{v}} 1.8$ en $\mathrm{Na}_{\mathrm{v}} 1.9$ is uit onderzoek gebleken dat ze een rol spelen bij pijn, één van de belangrijkste symptomen van DVN. Bij de mens zijn er mutaties in het $S C N$ gA-gen aangetoond in drie verschillende pijnsyndromen. Bij congenitale ongevoeligheid voor pijn werkt door de mutatie het kanaal niet meer (loss-of-function) en voelen patiënten helemaal geen pijn. Tegenovergesteld is er bij twee andere aandoeningen (primaire erythermalgie en paroxysmale-extreme-pijnsyndroom) juist een toename van de werking van 
het kanaal (gain-of-function), waardoor spontane pijn ontstaat. DVN vertoont in bepaalde opzichten overeenkomsten met deze twee ziektebeelden.

Om te bepalen of een verandering in een gen ook daadwerkelijk verantwoordelijk is voor de symptomen van de patiënt, kan gebruik worden gemaakt van functionele testen: 'patch-clamp' en 'current-clamp'- studies. Hiermee kunnen op celniveau de eigenschappen van het kanaal worden gemeten. Verschillende mutaties in het natriumkanaal-gen blijken tot verschillende veranderingen van de eigenschappen van dit natriumkanaal te leiden, waardoor de cellen verhoogd prikkelbaar zijn, langer geopend blijven of overmatig vuren (hyperexcitabiliteit). Mogelijk verklaart dit een deel van de verschillen in het klinisch beeld tussen de pijnsyndromen. Ook is gebleken dat het effect van een mutatie op de functie van het kanaal afhangt van de cellen waarin deze zich bevinden. Het is daarom belangrijk om mutaties te onderzoeken in de cellen waar ze klachten lijken te veroorzaken.

Hoofdstuk 1 geeft een overzicht van het ziektebeeld DVN en een uiteenzetting van het doel van dit proefschrift: onderzoeken of er mutaties in het bijzonder in het $\mathrm{SCN}_{9} A$-gen $\left(\mathrm{Na}_{\mathrm{v}} \mathrm{T} .7\right)$ voorkomen bij patiënten met DVN en nagaan of gevonden mutaties een rol kunnen spelen in de pathofysiologie van dit ziektebeeld. Dit om het klinische beeld beter te begrijpen en om mogelijkheden voor doelgerichte therapie voor de toekomst te creëren.

In hoofdstuk 2 wordt een uitgebreid literatuuroverzicht gegeven van de huidige kennis over DVN op het gebied van het stellen van de diagnose, de pathofysiologische mechanismen en de behandelopties. Er is nog geen gouden standaard voor het stellen van diagnose. Nieuwe diagnostische testen zouden het stellen van de diagnose in de toekomst kunnen optimaliseren, zoals bijvoorbeeld cornea confocale miscroscopie (tellen van zenuwvezels in het hoornvlies van de ogen met behulp van een microscoop) of nociceptieve evoked potentials (meten van geleiding van pijnprikkels naar de hersenen). $\mathrm{Er}$ wordt een overzicht gegeven van de ziektebeelden die zijn gerelateerd aan DVN. Ook komen nieuwe ontwikkelingen aan bod, die in de volgende hoofdstukken in detail worden beschreven.

Hoofdstuk 3 beschrijft het onderzoek dat is verricht bij een groep van 28 patiënten die voldeden aan de strikte criteria van idiopathische DVN (DVN zonder bekende oorzaak) naar afwijkingen in het $S C N_{9} A$-gen. Bij 8 patiënten $(29 \%)$ werd een (nieuwe) mutatie in het SCNgA-gen gevonden. Van deze 8 hadden 2 patiënten dezelfde mutatie. Deze mutaties werden vervolgens ingebouwd in menselijke embryonale niercellen en dorsale ganglioncellen voor elektrofysiologisch onderzoek. Het bleken 'gain-of-function' mutaties te zijn, 
waarbij overmatig vuren van de dorsale ganglioncellen werd aangetoond. Dit overmatige vuren kan pijnklachten en autonome klachten veroorzaken en zou daarnaast axonale degeneratie tot gevolg kunnen hebben, wat de afname van het aantal zenuwvezels in het huidbiopt bij deze patiënten zou kunnen verklaren.

In hoofdstuk 4 tot en met hoofdstuk 6 wordt aangetoond dat het effect van de mutatie op de functie van het kanaal afhangt van de cellen waarin deze onderzocht wordt.

Het klinisch beeld van 3 patiënten uit 2 (niet gerelateerde) families met DVN met $\mathrm{Na}_{\mathrm{v}} 1.7$ mutatie 1228M wordt beschreven in hoofdstuk 4 . Bij een van deze patiënten begonnen de klachten met pijn in het gelaat en bij een ander met pijn op de schedel. Naast functioneel onderzoek van de mutatie in menselijke embryonale niercellen en dorsale ganglioncellen, werden ook trigeminale ganglioncellen (verantwoordelijk voor het gevoel van het gezicht) onderzocht. Zowel in dorsale ganglioncellen als trigeminale ganglioncellen leidt de mutatie tot overmatig vuren, wat in relatie lijkt te staan tot het klachtenpatroon. Dit betekent ook dat het adagium van neuropathie als zenuwlengte-afhankelijke aandoening, begint in voeten en handen, niet altijd klopt.

De resultaten van de functionele studies van mutatie $1739 \mathrm{~V}$ komen in hoofdstuk 5 en hoofdstuk 6 aan bod. Deze mutatie blijkt niet alleen tot overmatig vuren van de dorsale ganglioncellen te leiden, maar daarnaast ook tot verlaagd vuren (hypopexcitatbiliteit) van bepaalde zenuwcellen van het autonome zenuwstelsel (sympathische neuronen van het bovenste cervicale ganglion). Dit heeft een 'loss-of-function' van het autonome zenuwstelsel tot gevolg. Dit is in overeenstemming met de uitgebreide autonome klachten die gezien worden bij de patiënten waarbij deze mutatie is aangetoond. Ter vergelijking is ook de functie van mutatie $\mathrm{R} 185 \mathrm{H}$ in de cervicale ganglioncellen onderzocht. Patiënten met deze mutatie hadden vrijwel geen autonome klachten. Zoals verwacht bracht deze mutatie geen veranderingen teweeg in dit type zenuwcellen.

In een familie waarbij drie gezinsleden $\mathrm{Na}_{\mathrm{v}} 1.7$ mutatie $\mathrm{G} 856 \mathrm{D}$ bleken te hebben, werden opvallende uiterlijke kenmerken gezien. Dit wordt beschreven in hoofdstuk 7. De patiënt, een broer en de vader hadden alle drie verkorte uiteinden van de ledematen (acromesomelia: kleine handen en voeten), roodheid van de extremiteiten, autonome klachten en pijn. De moeder en een andere broer zonder klachten waren beiden geen drager van de mutatie. Ook deze mutatie blijkt een verhoogde prikkelbaarheid en overmatig vuren van de cellen tot gevolg te hebben. Hoewel in het verleden nooit een specifiek verband tussen natriumkanaalmutaties en onderontwikkeling van de ledematen is beschreven, zijn er in de literatuur aanwijzingen dat botvorming en groei afhankelijk zijn van een goede functie van de dunne zenuwvezels. Bij deze mutatie is sprake van 
een functiestoornis in de dunne zenuwvezels die zeer vermoedelijk verstoorde botvorming en groei tot gevolg heeft.

Hoofdstuk 8 geeft een samenvatting van wat er tot op heden bekend is over de rol van $\mathrm{Na}_{\mathrm{v}}$.7 bij pijn. Het hoofdstuk beschrijft de resultaten uit dierenstudies en mensenstudies en besteedt in het bijzonder aandacht aan de effecten op celniveau van de gevonden mutaties bij de DVN-patiënten.

In hoofdstuk $\mathbf{9}$ worden de resultaten uit dit proefschrift ter discussie gesteld en aanbevelingen gedaan voor toekomstig onderzoek.

Het aantonen van $\mathrm{Na}_{\mathrm{v}} 1.7$ mutaties bij patiënten met idiopathische DVN zorgt ervoor dat patiënten meer zekerheid krijgen over de oorsprong van hun symptomen en geeft erkenning van hun ziektebeeld. Daarnaast dragen de bevindingen bij aan een verschil in denken over chronische neuropathische pijn. Het blijkt dat de uitkomsten van het onderzoek op celniveau vertaald kunnen worden naar het klinische beeld van patiënten. Dit is van belang bij het ontwikkelen van nieuwe doelgerichte therapieën.

Inmiddels zijn er op celniveau aanwijzingen gevonden dat de mutaties bijdragen aan een afname van het aantal dunne zenuwvezels in het huidbiopt, wat een van de diagnostische criteria van DVN is.

Met de toename van het aantonen van nieuwe mutaties zijn er efficiëntere technieken nodig om te kunnen analyseren of deze ook echt tot klachten kunnen leiden. Een model waarbij de zebravis wordt gebruikt, zou hiervoor mogelijk een goed middel kunnen zijn.

Verder onderzoek is nodig om na te gaan of er nog andere genen betrokken zijn bij het ontstaan van DVN. Daarnaast zouden natriumkanaalmutaties ook een rol kunnen spelen bij andere pijnlijke zenuwaandoeningen (neuropathieën), zoals diabetische neuropathie of chemotherapie-gerelateerde neuropathie. Maar ook bij andere aandoeningen met neuropathische pijn of ziektebeelden met onverklaarbare pijn is er mogelijk een plaats voor natriumkanaalmutaties. 


\section{Dankwoord}

Op de kaft van dit proefschrift staat alleen mijn naam. Dit boekje zou echter nooit tot stand zijn gekomen zonder het werk en de steun van vele anderen. Mijn dank is hiervoor groot. Ik wil graag een aantal personen in het bijzonder bedanken.

Allereerst gaat mijn dank uit naar mijn copromotoren Dr. I.S.J. Merkies en Dr. C.G. Faber. Jullie enthousiasme en betrokkenheid is ontzettend groot. Een fijner team om bij te mogen promoveren had ik me niet kunnen wensen. Ik hoop dat onze samenwerking nog lang mag blijven voortbestaan.

Beste Ingemar, bij jou liep ik mijn oudste coschap neurologie. Jij zag iets in mij, wat ik zelf nog niet had gezien. Gelukkig wist je me te overtuigen om bij jullie in Hoofddorp te komen werken. In de kliniek zag ik hoe ordelijk je bent. In de daaropvolgende samenwerking in de wetenschap ontdekte ik daarnaast je enorme creativiteit. Je hebt me laten zien dat niets onmogelijk is, zolang je er zelf maar in gelooft. Je weet me steeds weer opnieuw te stimuleren om het uiterste uit mezelf te halen. Ook al gaat dit soms met pittige discussies gepaard, ben ik je dankbaar voor je geduld, vele adviezen en steun.

Beste Karin, jij hebt ervoor gezorgd dat ik me meteen welkom voelde in Maastricht. Ik kan echt altijd bij je terecht. Je heerlijke noordelijke nuchterheid is ontzettend prettig. Je bent allergisch voor gezeur en houdt van aanpakken. Je vraagt om een grote inzet, maar dat is minstens ook wat je van jezelf verlangt. Dank dat je zo vaak laat merken dat je in mij gelooft. Je weet het altijd gezellig te maken en iedereen bij de groep te betrekken. Als ik je enthousiast aan het werk zie in de kliniek en de waardering van je patiënten ervaar, hoop ik dat ik ook zo'n fijne dokter mag worden.

Prof. Dr. R.J. Van Oostenbrugge, beste Robert, zowel in je rol van promotor als opleider, wil ik je bedanken voor je vertrouwen en de ondersteuning die je me de afgelopen jaren hebt gegeven. Small vessels of small fibers zijn beide ingrediënten voor interessante neurologie! Ik leer veel van je kritische blik en ben blij voor de prettige sfeer die er mede dankzij jou bij ons op de afdeling is.

Dear Prof. S.G. Waxman and Dr. S.D. Dib-Haji, it is a great honor for me that I had the opportunity to work with you and your fantastic team of Yale University. It was a pleasure to visit your laboratory to learn about the cell electrophysiological analysis of our variants. I truly thank you for the support and the belief you have expressed numerous times towards the Maastricht team. 
Dear Dr. G. Lauria, I would like to thank you and your team at Carlo Besta neurological institute in Milan for the wonderful collaboration through the years. It is nice to reinforce each other aiming to help more patients.

Bij de verdediging van dit proefschrift zullen Els Vanhoutte en Jessie Berends Van Genugten als paranimfen naast mij staan.

Lieve Els, zonder jou had ik nooit zo'n mooie start van mijn onderzoek gehad. Op iedere vraag die ik als beginnend onderzoeker had wist jij een antwoord. Je hebt meegeholpen met het onderzoeken van patiënten en hebt vele biopten geteld. Daarnaast was er gelukkig ook tijd voor gezelligheid, zoals bijvoorbeeld tijdens het PNS congres in Amerika of een training in Berlijn. Ik vind het hartstikke fijn dat je het nu naar je zin hebt bij de klinische genetica en hoop dat je daarnaast ook snel je boekje kunt gaan afronden.

Lieve Jessie, vanaf de eerste dag dat ik het azM binnenstapte hebben we een bijzondere vriendschap. We hoeven elkaar maar aan te kijken om te weten waar de ander aan denkt. Gelukkig mochten we tijdens ons perifere jaar samen naar Heerlen. Wat hebben we het gezellig gehad tijdens de vele autoritjes! Ook buiten het werk hebben we veel lol samen. Bedankt voor de mooie momenten die we al samen hebben mogen beleven. Ik hoop dat er nog velen zullen volgen.

Naast mijn promotieteam, zijn er nog een aantal mensen uit de neuromusculaire groep die ik graag speciaal zou willen bedanken. Als eerste Mayienne Bakkers. Beste Mayienne, zonder al jouw werk zou het nooit mogelijk zijn geweest om zo geordend onze patiënten te kunnen selecteren. Bedankt voor de moeite die je erin hebt gestoken om mij biopten te leren tellen. Jij zult echter altijd de koningin van de huidbiopten blijven! Succes met het afronden van je proefschrift.

Daarnaast wil ik onze fantastische neuromusculair verpleegkundig specialist Carla Gorissen-Brouwers bedanken. Lieve Carla, ik kan me nu al niet meer voorstellen hoe we alles geregeld kregen voordat jij er was. Jij bent er altijd voor de patiënt, maar ook voor ons. Je bent een grote aanwinst voor ons team! Eveneens wil ik je collega verpleegkundig specialisten Anouk van der Kwaak en Mirella Davies Waber bedanken, omdat zij ook regelmatig met veel toewijding patiënten met de verdenking op een dunnevezelneuropathie op de neurodaycare hebben gezien.

Zonder Charlotte van Hoegee en Edith Peeters zouden onze patiënten nooit op de juiste tijd op de juiste plaats zijn. Zij zorgen ervoor dat alles voor onze patiënten op rolletjes loopt. Bedankt dat jullie altijd bereid zijn om dingen te regelen, ook als ik weer eens een onmogelijk verzoek heb om een patiënt op korte termijn te willen zien.

Tenslotte wil ik Mieke Hermans bedanken voor alle tips die ze mij heeft gegeven 
om het afronden van dit boekje succesvol te laten verlopen. Zelfs tijdens je zwangerschapsverlof kon ik je nog lastig vallen, bedankt daarvoor!

Dit proefschrift is niet alleen het werk van mensen van de afdeling neurologie. Op het moment dat wij weer eens vele huidbiopten hadden afgenomen, wist de afdeling pathologie deze altijd netjes te verwerken. Ik wil hiervoor in het bijzonder Benoit Frere, Ilse Driesmans en Aline Kosten bedanken.

De afdeling Clinical Genomics heeft tevens een zeer grote bijdrage aan dit onderzoek geleverd. Als eerste gaat er veel dank uit naar Monique Gerrits die samen met Diane Merckx en Linda Meekels heeft gezorgd voor de analyses van de gevonden varianten. Beste Monique, wat fijn dat je altijd bereikbaar bent voor overleg en de tijd neemt om uit te leggen hoe iets zit als mijn genetische kennis tekort schiet. Je weet gezelligheid goed te combineren met hard werken. Daarnaast wil ik het hoofd van de afdeling, Prof. Dr. H.J.M. Smeets speciaal bedanken voor de ondersteuning die hij heeft gegeven, waarbij hij onze klinische ideeën via genetische lijnen tastbaar heeft gemaakt.

Daarnaast gaat ook dank uit naar de medewerkers van de afdeling klinische neurofysiologie. Zij hebben regelmatig met hun planning moeten stoeien om ervoor te zorgen dat onze patiënten hun onderzoeken op dezelfde dag konden ondergaan.

Gelukkig heb ik de afgelopen 4 jaar wetenschap met de kliniek kunnen combineren. Dit was niet mogelijk geweest zonder de ondersteuning van alle artsassistenten en stafleden van de afdeling neurologie en klinische neurofysiologie. Ik ben dankbaar dat ik deel mag uitmaken van zo'n fijne groep. Extra dank gaat uit naar mijn drie jaargenoten Ellen, Jessie en Laura, die naast collega's tevens vriendinnen zijn geworden.

Ook tijdens mijn perifere stage in Heerlen heb ik de ruimte gekregen om patiënten voor mijn onderzoek te blijven zien. Ik wil daarom hiervoor de arts-assistenten en stafleden van de vakgroep neurologie van het Atrium MC bedanken en voor de interesse die zij altijd in de studie hebben getoond.

Speciale dank gaat uit naar alle patiënten en hun familieleden die bereid zijn geweest om aan dit onderzoek deel te nemen. Uit het gehele land zijn mensen naar Maastricht gekomen om onderzoeken te ondergaan. Daarnaast werden er vele vragenlijsten verzameld en regelmatig telefoontjes gepleegd om zoveel mogelijk informatie te verkrijgen. Met het afronden van dit proefschrift is het onderzoek nog lang niet klaar. Het is slechts het begin van de zoektocht naar betere therapieën. 
Spierziekten Nederland en het Prinses Beatrix Spierfonds wil ik bedanken voor hun ondersteuning en de erkenning van de aandoening dunnevezelneuropathie. Dit is ontzettend belangrijk voor onze patiënten die voorheen niet wisten waar ze naar toe moesten gaan met hun vragen.

Ik wil Annette Jonkhoff bedanken voor het geven van commentaar op mijn Nederlandse samenvatting en de tips om mijn tekst in dit boekje meer overzichtelijk te maken.

Het harde werken moet soms worden afgewisseld met ontspanning. Gelukkig kan ik daarvoor altijd bij mijn 'Amsterdam-vriendinnen' en hun mannen terecht! Lieve Anna en Bastiaan, Hester en Jorge, Mariëtte en Jordy, Petra en Aidan, Ronni en Gal, Suzanne en Erwin, ook al zijn we helemaal naar de andere kant van het land verhuisd, als we bij elkaar zijn is het altijd weer feest. Wat hebben we het leuk gehad tijdens onze 'huwelijksreizen' naar Israël en Mexico. Ondanks dat niemand van jullie voor de specialisatie neurologie heeft gekozen, weten jullie geloof ik beter dan menig neuroloog wat een dunnevezelneuropathie is! Bedankt dat jullie het snappen als ik even een feestje in de Randstad oversla.

In dit boekje staan twee plaatjes van natriumkanalen die door één van mijn middelbare school vriendinnen, Kim Rutten, zijn gemaakt. De 'Body Builder' plaatjes zijn helaas net niet door de selectie gekomen. Toch bedankt voor al het werk dat je erin hebt gestoken! Ik zal ze een plekje aan de muur geven!

Natuurlijk wil ik ook mijn familie bedanken. Lieve pap en mam, ik ben jullie dankbaar voor het fijne thuis dat er altijd bij jullie is en de ondersteuning die jullie me altijd hebben gegeven in alles wat ik doe. Hopelijk wordt met dit boekje en de verdediging een beetje duidelijk waar ik toch altijd zo druk mee bezig ben geweest. Lieve Sjoerd, we zijn allebei altijd heel verschillend geweest, maar beiden hebben we onze eigen dingen bereikt waar we trots op mogen zijn. Het is altijd fijn om bij jou, Marjo, Mathies en Lente op visite te komen. Lieve Oma Hoeijmakers, wat vind ik het super knap dat je met je 90 jaar nog precies weet waar je kinderen en (achter)kleinkinderen zich mee bezig houden. Dit boekje hoef je echt niet helemaal te lezen hoor, je doet al genoeg voor ons! Van mijn ooms en tantes, neven en nichten, wil ik in het bijzonder mijn neef Gijs bedanken. Ondanks dat je helemaal niet medisch bent, heb je een mooie passende kaft ontworpen. Ik ben er heel erg blij mee!

Beste Jan en Ria, door de verhuizing naar Maastricht is de afstand naar Drenthe nog een stuk groter geworden. Helaas ben ik daardoor wat minder vaak bij jullie dan ik eigenlijk zou willen. Ik wil jullie bedanken voor het begrip hiervoor 
en de gastvrijheid die er altijd is als ik bij jullie ben. Wim, misschien kunnen wij ook wel een keer samen een artikel schrijven. Je bent altijd met Caroline bij ons welkom om door de heuvels te komen fietsen.

Lieve Peter, we hebben al vaak grapjes gemaakt over hoe ik jou zou gaan bedanken. Bijvoorbeeld dat je blij mag zijn dat ik zo vaak met mijn laptop op schoot zit, zodat jij lekker voetbal kan kijken. Maar wat heb ik het getroffen met jou! Veel mannen zouden al lang gek zijn geworden van mij, maar wij laten elkaar gelukkig vrij in de dingen die we leuk vinden. Ik vind het knap dat je naast je drukke baan een extra opleiding bent gaan volgen en dat ondanks dat je als Drent helemaal naar Maastricht bent verhuisd, je het hier toch zo naar je zin hebt gemaakt. Ik hoop dat we het nog lang samen fijn mogen hebben. Bedankt voor alles en erg veel liefs! 


\section{Curriculum Vitae}

Janneke Gertrude Jacobine Hoeijmakers was born on June 29th, 1983 in Venray, the Netherlands. In 2001 she graduated from highschool (Gymnasium, Dendron College, Horst). In the same year she started with the Health Science education at the University of Maastricht. After she obtained her first-year certificate, she moved to Amsterdam to start her medical training at the VU University. In 2008, she attended her final internship at the neurology department Spaarne Hospital in Hoofddorp, under supervision of Dr. I.S.J. Merkies. After she obtained her medical doctor degree, she started her working career as resident neurology in this hospital. In June 2009 she moved back to Maastricht to work at the neurology department of the Maastricht University Medical Center and started her neurology training in January 2010 (Dr. M.C.T.F.M. De Krom, Prof. Dr. R.J. Van Oostenbrugge). At the same time, she started her PhD program investigating the role of sodium channels in small fiber neuropathy under supervision of Dr. C.G. Faber en Dr. I.S.J. Merkies, in close collaborationship with S. G. Waxman, Bridget Flaherty Professor of Neurology, Neurobiology, and Pharmacology at Yale University and Director of the Neuroscience \& Regeneration Research Center at Yale University School of Medicine, New Haven, USA and Dr. G Lauria, neurologist at the Neuromuscular Diseases Unit, IRCCS Foundation of the Carlo Besta Instituto, Milan, Italy. In January 2012 she was awarded with the Prinses Beatrix Spierfonds year prize, for the Annals of Neurology paper 'Gain of function $\mathrm{Na}_{\mathrm{v}} 1.7$ mutations in idiopathic small fiber neuropathy'. The same paper has been granted with the 2013 Annals of Neurology Prize 'for a distinguished contribution to clinical neuroscience'. In addition, she was awarded for the Nature Review Neurology paper 'Small-fibre neuropathies-advances in diagnosis, pathophysiology and management' as the Best Paper, on the School for Mental Health and Neuroscience (MHeNS) research day in 2013. In January 2016 she will finish her neurology training.

Janneke Gertrude Jacobine Hoeijmakers werd geboren op 29 juni 1983 in Venray. In 2001 behaalde ze haar gymnasium diploma aan het Dendron College te Horst. In datzelfde jaar begon ze met de opleiding Gezondheidswetenschappen aan de Universiteit van Maastricht. Nadat zij haar propedeuse had behaald verhuisde ze naar Amsterdam, waar ze begon met de studie Geneeskunde aan de Vrije Universiteit. Aan het einde van haar opleiding in 2008 , volgde ze haar laatste co-schap op de afdeling neurologie van het Spaarne Ziekenhuis in Hoofddorp onder begeleiding van Dr. I.S.J. Merkies. Nadat ze haar artsenbul had behaald, bleef zij in dit ziekenhuis werken als arts-assistent neurologie. In juni 2009 
verhuisde ze terug naar Maastricht om te gaan werken op de neurologie afdeling van het Maastrichts Universitair Medisch Centrum, waar ze haar opleiding tot neuroloog startte in januari 2010 (Dr. M.C.T.F.M. De Krom, Prof. Dr. R.J. Van Oostenbrugge). Tegelijkertijd begon ze met haar onderzoekstraject over de rol van natriumkanalen bij dunnevezelneuropathie onder supervisie van Dr. C.G. Faber en Dr. I.S.J. Merkies, en in een nauw samenwerkingsverband met S. G. Waxman, Bridget Flaherty Professor of Neurology, Neurobiology, en Pharmacology aan de Yale University en Director of the Neuroscience \& Regeneration Research Center aan de Yale University School of Medicine, New Haven, USA en Dr. G Lauria, neuroloog aan de Neuromuscular Diseases Unit, IRCCS Foundation of the Carlo Besta Instituto, Milaan, Italië. In januari 2012 werd ze onderscheiden met de Prinses Beatrix Spierfonds jaarprijs, voor het Annals of Neurogy artikel 'Gain of function $\mathrm{Na}_{\mathrm{v}} 1.7$ mutations in idiopathic small fiber neuropathy', als het beste neuromusculaire artikel gepubliceerd in 2012. Ditzelfde artikel is bekroond met de 2013 Annals of Neurology Prize, voor een onderscheidende bijdrage aan de klinische neurowetenschappen. Daarnaast, heeft ze voor het Nature Review Neurology artikel 'Small-fibre neuropathies-advances in diagnosis, pathophysiology and management', de prijs voor beste paper ontvangen op de onderzoeksdag van de School for Mental Health and Neuroscience (MHeNS) in 2013. In januari 2016 zal zij haar opleiding tot neuroloog afronden. 


\section{List of publications}

Han, C., Vasylyev, D., Macala, L.J., Gerrits, M.M., Hoeijmakers, J.G.J., Bekelaar, K.J., Dib-Hajj, S.D., Faber, C.G., Merkies, I.S.J. \& Waxman, S.G. The G1662S Na 1.8 mutation in small fibre neuropathy: impaired inactivation underlying DRG neuron hyperexcitability. J Neurol Neurosurg Psychiatry (2013). doi: 10.1136/jnnp-2013-306095.

Peters, M.J.H., Bakkers, M., Merkies, I.S.J., Hoeijmakers, J.G.J., van Raak, E.P.M. \& Faber, C.G. Incidence and prevalence of small-fiber neuropathy: A survey in the Netherlands. Neurology 81, 1356-60 (2013).

Huang, J., Yang, Y., Zhao, P., Gerrits, M.M., Hoeijmakers, J.G.J., Bekelaar, K.J., Merkies, I.S.J., Faber, C.G., Dib-Hajj, S.D. \& Waxman, S.G. Small-fiber neuropathy $\mathrm{Na}_{\mathrm{v}} 1.8$ mutation shifts activation to hyperpolarized potentials and increases excitability of dorsal root ganglion neurons. J Neurosci 33, 14087-97 (2013).

Black, J.A.*, Hoeijmakers, J.G.J.*, Faber, C.G., Merkies, I.S.J. \& Waxman, S.G. $\mathrm{NaV}$ 1.7: stress-induced changes in immunoreactivity within magnocellular neurosecretory neurons of the supraoptic nucleus. Mol Pain 9, 39 (2013). * co-first authorship

Bakkers, M., Faber, C.G., Hoeijmakers, J.G.J., Lauria, G. \& Merkies, I.S.J. Small fibers, large impact: Quality of life in small-fiber neuropathy. Muscle Nerve (2013). doi: 10.1002/mus.23910.

Faber, C.G., Lauria, G., Merkies, I.S.J., Cheng, X., Han, C., Ahn, H.S., Persson, A.K., Hoeijmakers, J.G.J., Gerrits, M.M., Pierro, T., Lombardi, R., Kapetis, D., Dib-Hajj, S.D. \& Waxman, S.G. Gain-of-function $\mathrm{Na}_{\mathrm{v}} 1.8$ mutations in painful neuropathy. Proc Natl Acad Sci U S A 109, 19444-9 (2012).

Han, C., Hoeijmakers, J.G.J., Liu, S., Gerrits, M.M., te Morsche, R.H.M., Lauria, G., Dib-Hajj, S.D., Drenth, J.P.H., Faber, C.G., Merkies, I.S.J. \& Waxman, S.G. Functional profiles of SCNgA variants in dorsal root ganglion neurons and superior cervical ganglion neurons correlate with autonomic symptoms in small fibre neuropathy. Brain 135, 2613-28 (2012).

Hoeijmakers, J.G.J., Merkies, I.S.J., Gerrits, M.M., Waxman, S.G. \& Faber, C.G. Genetic aspects of sodium channelopathy in small fiber neuropathy. Clin Genet 82, 351-8 (2012).

Hoeijmakers, J.G.J., Faber, C.G., Lauria, G., Merkies, I.S.J. \& Waxman, S.G. Small-fibre neuropathies--advances in diagnosis, pathophysiology and management. Nat Rev Neurol 8, 369-79 (2012). 
Han, C.*, Hoeijmakers, J.G.J.*, Ahn, H.S., Zhao, P., Shah, P., Lauria, G., Gerrits, M.M., te Morsche, R.H.M., Dib-Hajj, S.D., Drenth, J.P.H., Faber, C.G., Merkies, I.S.J. \& Waxman, S.G. Na 1 1.7-related small fiber neuropathy: impaired slow-inactivation and DRG neuron hyperexcitability. Neurology 78, 1635-43 (2012). * co-first authorship

Hoeijmakers, J.G.J., Bakkers, M., Blom, E.W., Drenth, J.P.H., Merkies, I.S.J. \& Faber, C.G. Dunnevezelneuropathie: kennen is herkennen. Ned Tijdschr Geneeskd 156, A4224 (2012).

Hoeijmakers, J.G.J., Han, C., Merkies, I.S.J., Macala, L.J., Lauria, G., Gerrits, M.M., Dib-Hajj, S.D., Faber, C.G. \& Waxman, S.G. Small nerve fibres, small hands and small feet: a new syndrome of pain, dysautonomia and acromesomelia in a kindred with a novel $\mathrm{Na}_{\mathrm{v}} \mathrm{T} .7$ mutation. Brain $135,345-58$ (2012).

Estacion, M., Han, C., Choi, J.S., Hoeijmakers, J.G.J., Lauria, G., Drenth, J.P.H., Gerrits, M.M., Dib-Hajj, S.D., Faber, C.G., Merkies, I.S.J. \& Waxman, S.G. Intra- and interfamily phenotypic diversity in pain syndromes associated with a gain-of-function variant of $\mathrm{NaV}$ 1.7. Mol Pain 7, 92 (2011).

Faber, C.G.*, Hoeijmakers, J.G.J.*, Ahn, H.S., Cheng, X., Han, C., Choi, J.S., Estacion, M., Lauria, G., Vanhoutte, E.K., Gerrits, M.M., Dib-Hajj, S., Drenth, J.P.H., Waxman, S.G. \& Merkies, I.S.J. Gain of function $\mathrm{Na}_{\mathrm{v}} 1.7$ mutations in idiopathic small fiber neuropathy. Ann Neurol 71, 26-39 (2012). * co-first authorship

van Nispen, R.M.A., de Boer, M.R., Hoeijmakers, J.G.J., Ringens, P.J. \& van Rens, G.H.M.B. Co-morbidity and visual acuity are risk factors for healthrelated quality of life decline: five-month follow-up EQ-5D data of visually impaired older patients. Health Qual Life Outcomes 7, 18 (2009). 


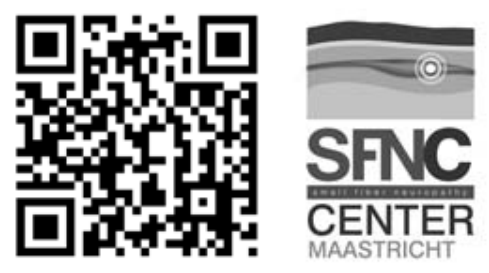

For the digital version of this thesis and more information about small fiber neuropathy, visit: www.smallfiberneuropathy.com

Voor de digitale versie van dit proefschrift en meer informatie over dunnevezelneuropathie, bezoek: www.dunnevezelneuropathie.n 